NBSIR 79-1789

\title{
Economic Analysis of Insulation in Selected Masonry and Wood-Frame Walls
}

Stephen R. Petersen

Building Economics and Regulatory Technology Division

Center for Building Technology

National Engineering Laboratory

National Bureau of Standards

September 1979

\section{Prepared for}

Dffice of Policy Development and Research 



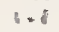

\section{ECONOMIC ANALYSIS OF INSULATION IN SELECTED MASONRY AND WOOD-FRAME WALLS}

Stephen R. Petersen

Building Economics and Regulatory Technology Division Center for Building Technology National Engineering Laboratory National Bureau of Standards

Prepared for

Office of Policy Development and Research

U.S. Department of Housing and Urban Development

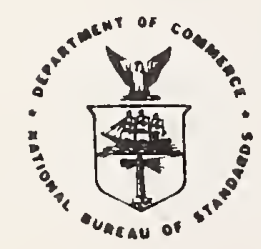

U.S. DEPARTMENT OF COMMERCE, Juanita M. Kreps, Secretary Luther H. Hodges, Jr., Under Secretary

Jordan J. Baruch. Assistant Secretary for Science and Technology

NATIONAL BUREAU OF STANDARDS, Ernest Ambler, Director 


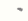




\section{Preface}

This National Bureau of Standards Interagency Report is the result of a request from U.S. Department of Housing and Urban Development under Interagency Agreement H-24-77 (Quick Response Studies), Tasks 13 and 19. It builds directly on concurrent research in economic analysis of insulation in masonry walls funded by the Department of Energy under Interagency Agreement NO. 77-A-01-6010, Task Order A008, BCS. 


\section{ACKOWLEDGEMENTS}

The author wishes to acknowledge the valuable contribution to this report provided by Bradley Peavy of the Building Thermal and Service Systems Division, Center for Building Technology, in making the NBSLD computer runs for this report. In addition, Kimberly Hockenbery provided great ly appreciated assistance in making computer files and computer runs for the economic analysis and in helping assemble the final draft version of this report. Hans J. Milton assisted in smoothing the transition to metric equivalents. I also wish to thank James G. Gross, Paul R. Achenbach, Lawrence E. Flynn and Robert D. Dikkers for their timely review of the final manuscript. 
LIST OF TABLES ................................ vi

LIST OF FIGURES .............................. vii

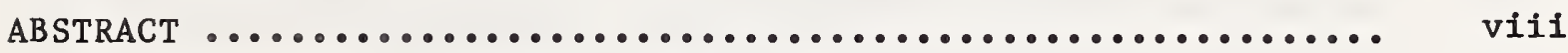

1.0 INTRODUCTION $\ldots \ldots \ldots \ldots \ldots \ldots \ldots \ldots \ldots \ldots \ldots \ldots \ldots \ldots \ldots \ldots \ldots \ldots \ldots$

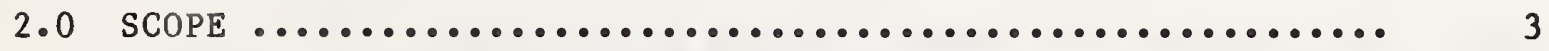

3.0 MODEL ING THE WALlS FOR THERMAL ANALYSIS .............. 8

4.0 TECHNIQUES OF ECONOMIC ANALYSIS $\ldots \ldots \ldots \ldots \ldots \ldots \ldots \ldots \ldots \ldots \ldots . \ldots \ldots$

5.0 RESULTS OF THERMAL AND ECONOMIC ANALYSIS .............. 39

6.0 CONCLUSIONS $\ldots \ldots \ldots \ldots \ldots \ldots \ldots \ldots \ldots \ldots \ldots \ldots \ldots \ldots \ldots \ldots \ldots \ldots \ldots$

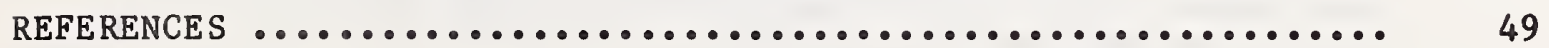

APPENDIX A Computer Printouts of Economic Analyses for A11 Cities Assuming No Cooling Savings ........... A-1

APPENDIX B Computer Printouts of Economic Analyses for Tampa, Phoenix and Jacksonville -- Cooling Savings Based on Windows Closed ................ B-1

APPENDIX C Computer Printouts of Economic Analyses for Tampa, Phoenix and Jacksonville -- Cooling Savings Based on Cooling above $78^{\circ} \mathrm{F}\left(25.5^{\circ} \mathrm{C}\right)$ Outdoor Temperature ........................ C-1

APPENDIX D Computer Printouts of Economic Analyses for Madison, Phoenix and Washington, D.C., Assuming $90^{\circ}$ Rotation of Building ................... D-1 


\section{LIST OF TABLES}

1* Component and Total Resistance for Wall Components and Walls to be Examined ....................... 4

2 Cities, Degree Days, Shell Specifications ............ 7

3 Prototypical House Parameters .................... 7

4 Operational Profile of House .................... 10

$5 A^{*} \quad$ Wall U-values and Annual Heating and Cooling Requirements for $1176 \mathrm{ft}^{2}$ House ( $8^{\prime \prime}$ Block

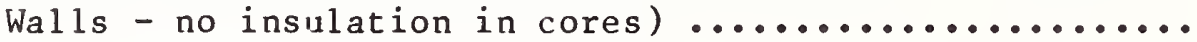

5B* Wall U-values and Annual Heating and Cooling Requirements for $1176 \mathrm{ft}^{2}$ House ( 8 " Block Walls - perlite insulation in cores) ................ 14

5C* Wall U-values and Annual Heating and Cooling Requirements for $1176 \mathrm{ft}^{2}$ House (Wood-frame

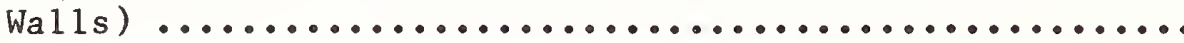

5D* Wall U-values and Annual Heating and Cooling Requirements for $1176 \mathrm{ft}^{2}$ House-90 Rotation ( $8^{\prime \prime}$ B lock

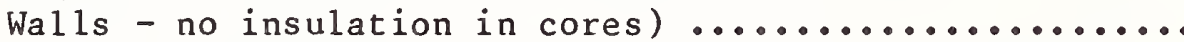

6 Normalized Annual Heating and Cooling Requirements in million Btu $(G J)$, Wall $U=0.10$ (U-metric $=0.57) \ldots 30$

7 Heating and Cooling System Efficiencies (Seasonal)..... 33

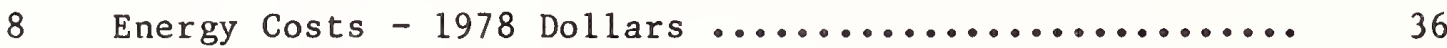

9 Present Worth Factors for Energy Savings ............ 36

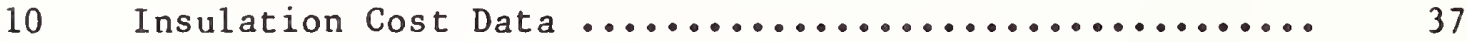

11 Optimal Insulation Level and Net Savings by Furnace Type, City, and Insulation Group (Insulation Prices based on Table 10) $\ldots \ldots \ldots \ldots \ldots \ldots \ldots \ldots \ldots \ldots$

12 Optimal Insulation Level and Net Savings by Furnace Type, City, and Insulation Group (Insulation

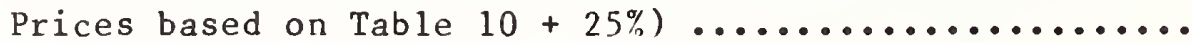

* An equivalent table expressed in metric (SI) units immediately follows. 


\section{LIST OF FIGURES}

1 Annual Heating and Cooling Requirements by Wall

U-value - Albuquerque, New Mexico ..................

2 Annual Heating and Cooling Requirements by Wall

U-value - Atlanta, Georgia ......................

3 Annual Heating and Cooling Requirements by Wall

U-value - Indianapolis, Indiana ..................

4 Annual Heating and Cooling Requirements by Wall

U-value - Jacksonville, Florida ..................

5 Annual Heating and Cooling Requirements by Wa11

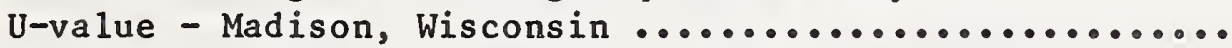

6 Annual Heating and Cooling Requirements by Wall

U-value - Phoenix, Arizona

7 Annual Heating and Cooling Requirements by Wall

U-value - Tampa, Florida

8 Annual Heating and Cooling Requirements by Wa11

U-value - Washington, D.C. 
This report provides a life-cycle cost-benefit analysis of several alternative methods for insulating $8^{\prime \prime}(200 \mathrm{~mm})$ concrete masonry walls in new single-family residences. In addition, a cost-benefit analysis for insulation in wood-frame walls is provided, consistent with the assumptions used in the masonry wall analysis. A dynamic load simulation mode1, NBSLD, was used to calculate the heating and cooling requirements for a 1176 square foot $\left(110 \mathrm{~m}^{2}\right)$ house with different levels of thermal resistance for both wall types in eight geographic locations. These data are used to calculate the reduction in annual heating and cooling requirements due to several different types of insulation in the cores and furring spaces of the masonry wall and the cavities of the wood-frame wall.

Economic analysis is applied to determine estimates of , life-cycle savings from insulation for different locations and furnace types in order to determine the most cost-effective insulation level. In general it is found that the maximum economically justifiable level of insulation in the masonry wall is considerably lower than for the wood-frame wall because of the significantly higher cost of insulating masonry walls.

Key words: Building economics; energy conservation; insulation; life-cycle cost analysis; masonry construction; space heating and cooling requirements; thermal mass. 


\subsection{INTRODUCTION}

The Center for Building Technology at the National Bureau of Standards is currently conducting research on the thermal performance of exterior walls of residential buildings, with emphasis on masonry wall construction, in order to assess the economic benefits and costs of alternative insulation strategies. * This research has been sponsored by the Department of Energy (DoE). The Department of Housing and Urban Development-Federal Housing Administration (HUD-FHA) has supported one phase of this study to provide information needed in the development of their new Minimum Property Standards (MPS) for residential buildings. This report covers that phase of the study.

This report provides specific information requested by HUD-FHA, along with the basic methodology and data used to generate these results. Further description of the methodology and more extensive data and results will be made available upon the completion of the overall research project and publication of the final research report for DoE.

The major objectives of the NBS research in insulation strategies for exterior walls are to determine the extent to which insulation in alternative wall configurations is cost effective on a life-cycle basis; to develop insulation guidelines based on a life-cycle-cost-minimization objective; and to establish the sensitivity of these guidelines to climate, fuel cost and insulation costs on a local basis. These objectives

* Note: Metric (SI) Units have been included in this report for reference purposes, in line with the policy of the National Bureau of Standards. Rounding has been applied to reflect equivalent precision. 
are consistent with recent policy decisions of HUD-FHA in updating their MPS for single family residences based on the life-cycle-cost effectiveness of a number of conservation measures for reducing space heating and cooling requirements. Moreover, they are consistent with recent DoE decisions regarding the use of economic analysis in the development of building energy performance standards for buildings.

Cost-effective strategies for increasing the thermal performance of wood-frame and masonry walls in the same climate and for the same cost per Btu of heat delivered to (or removed from) the conditioned space might be expected to differ for two distinct reasons: (1) the reduction in annual heating and cooling requirements due to an equal decrease in $\mathrm{U}$-value for the walls may be different and (2) the cost of reducing the U-value for these two wall types may be different.

The first reason is related to the thermal response, or time-delay properties, of the heavier wall which tends to dampen the effects of climate on space cooling and heating requirements. The extent to which this affects the savings from an equal decrease in U-value will be seen later in this report. The second reason is a result of the $3-1 / 2^{\prime \prime}(89 \mathrm{~mm}) \mathrm{stud}$ space in the wood-frame wall which provides a greater space for low-cost insulation materials than the typical $3 / 4^{\prime \prime}(19 \mathrm{~mm})$ furring space, and, in some cases, open cores, of a masonry wall. In order to minimize the reduction of usable floor space due to higher levels of insulation on the inside of the dwelling unit, low conductivity, but relatively expensive (e.g., polystyrene and isocyanurate) insulation materials are often used in masonry walls at a considerable cost difference over typical 
insulating materials used in wood-frame construction (e.g., fiberglass blanket). Alternatively, wood framing can be added inside the masonry wall together with more conventional fiberglass insulation materials. However, the increased cost of both the framing and the extension of end walls to maintain an equivalent inside floor area must be incorporated into the cost analysis. Insulation on the outside of the exterior walls, while somewhat superior from a thermal performance is not as cost effective as insulation on the inside, since a protective covering must be installed. Such a covering would also negate the benefit of low exterior maintenance characteristic of masonry materials. (Insulation in the cavity of a brick and block cavity wall is not considered in this report although it will be examined in a subsequent, more comprehensive report to DoE.)

\section{$2.0 \underline{\text { SCOPE }}$}

HUD-FHA has requested that NBS examine the cost effectiveness of several alternative insulation strategies for masonry walls. In particular, reflective air spaces, rigid isocyanurate insulation, and rigid polystyrene insulation are to be examined with and without core insulation (perlite) in an $8^{\prime \prime}(200 \mathrm{~mm})$ block wall with $3 / 4^{\prime \prime}(19 \mathrm{~mm})$ furring strips and 1/2" (13 mm) gypsum wallboard on the inside. The exact specifications of the insulation strategies to be examined, as specified by HUD-FHA, are shown in Table 1. There are nine different insulation systems specified, for use with $8^{\prime \prime}(200 \mathrm{~mm})$ block walls. In addition, the sponsor asked NBS to evaluate the cost effectiveness of $R-11, R-13$ 
Table 1. Component and Total Resistance ${ }^{a}$ for Wall Components and Walls to be Examined Wa11-Type Designators

\begin{tabular}{|c|c|c|c|c|c|c|c|c|c|}
\hline Construction & B3 & B4 & C3 & $\mathrm{C4}$ & D3 & D4 & F2 & F5 & EXTRA \\
\hline 100 Pcf $\mathrm{CMU}^{\mathrm{C}}$ & 1.75 & & 1.75 & & 1.75 & & 1.75 & & 1.75 \\
\hline Cores filled (Perlite) & & 4.85 & & 4.85 & & 4.85 & & 4.85 & \\
\hline 3/4" Reflective Space & 3.48 & 3.48 & & & & & & & \\
\hline $1^{\circ "}$ Reflective Space & & & & & & & & & 3.51 \\
\hline $1 / 2^{\prime \prime}$ Isocyanurate & & & & & & & & & 4.00 \\
\hline $3 / 4^{\prime \prime}$ Isocyanurate & & & 6.00 & 6.00 & & & & & \\
\hline $3 / 4^{\prime \prime}$ Polystyrene & & & & & 3.94 & 3.94 & & & \\
\hline $1-1 / 2 "$ Polystyrene & & & & & & & 7.89 & 7.89 & \\
\hline 1/2" Gypsum board & 0.45 & 0.45 & 0.45 & 0.45 & 0.45 & 0.45 & 0.45 & 0.45 & 0.45 \\
\hline Outside Surface Air & 0.17 & 0.17 & 0.17 & 0.17 & 0.17 & 0.17 & 0.17 & 0.17 & 0.17 \\
\hline Inside Surface Air & 0.68 & 0.68 & 0.68 & 0.68 & 0.68 & 0.68 & 0.68 & 0.68 & 0.68 \\
\hline Total $\mathrm{R}$ & 6.53 & 9.63 & 9.05 & 12.15 & 6.99 & 10.09 & 10.94 & 14.04 & 10.56 \\
\hline $1 / R=U$ & 0.153 & 0.104 & 0.110 & 0.082 & 0.143 & 0.099 & 0.091 & 0.071 & 0.095 \\
\hline
\end{tabular}

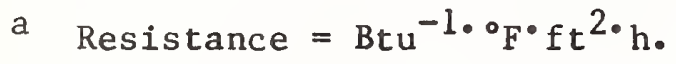

b As specified by HUD-FHA.

c Concrete masonry unit: 8" hollow block, 100 pcf density concrete
} 
Table 1-M. Component and Total Resistance ${ }^{a}$ for Wall Components and Walls to be Examined Wall-Type Designators

\begin{tabular}{|c|c|c|c|c|c|c|c|c|c|}
\hline Construction & B3 & B4 & $\mathrm{C} 3$ & $\mathrm{C} 4$ & D3 & D4 & F2 & F5 & EXTRA \\
\hline $1600 \mathrm{~kg} / \mathrm{m}^{3} \mathrm{CMU}^{3}$ & 0.31 & & 0.31 & & 0.31 & & 0.31 & & 0.31 \\
\hline Cores filled (Perlite) & & 0.85 & & 0.85 & & 0.85 & & 0.85 & \\
\hline $19 \mathrm{~mm}$ Reflective Space & 0.61 & 0.61 & & & & & & & \\
\hline $25 \mathrm{~mm}$ Reflective Space & & - & & & & & & & 0.62 \\
\hline $13 \mathrm{~mm}$ Isocyanurate & & & & & & & & & 0.70 \\
\hline $19 \mathrm{~mm}$ Isocyanurate & & & 1.06 & 1.06 & & & & & \\
\hline $19 \mathrm{~mm}$ Polystyrene & & & & & 0.69 & 0.69 & & & \\
\hline $38 \mathrm{~mm}$ Polystyrene & & & & & & & 1. 39 & 1.39 & \\
\hline $13 \mathrm{~mm}$ Gypsum board & 0.08 & 0.08 & 0.08 & 0.08 & 0.08 & 0.08 & 0.08 & 0.08 & 0.08 \\
\hline Outside Surface Air & 0.03 & 0.03 & 0.03 & 0.03 & 0.03 & 0.03 & 0.03 & 0.03 & 0.03 \\
\hline Inside Surface Air & 0.12 & 0.12 & 0.12 & 0.12 & 0.12 & 0.12 & 0.12 & 0.12 & 0.12 \\
\hline Total R & 1.15 & 0.70 & 1.60 & 2.14 & 1.23 & 1.78 & 1.93 & 2.47 & 1.86 \\
\hline $1 / R=U$ & 0.87 & 0.59 & 0.63 & 0.47 & 0.84 & 0.56 & 0.52 & 0.40 & 0.54 \\
\hline
\end{tabular}
a Resistance $=\mathrm{m}^{2} \cdot \mathrm{K} / \mathrm{W}$
$b$ As specified by HUD-FHA.
c Concrete masonry unit: $200 \mathrm{~mm}$ hollow block, $1600 \mathrm{~kg} / \mathrm{m}^{3}$ density concrete 
and $R-19$ (R-metric: $1.9,2.3$ and 3.3) insulation in wood-frame walls, using the same basic assumptions and modeling procedures. Composite or cavity brick and block walls, which are more common than $8^{\prime \prime}(200 \mathrm{~mm})$ block walls in most parts of the U.S. other than Florida and the Southwest, are not examined in this report.

Previous NBS analysis of the cost effectiveness of a number of conservation measures in wood-frame housing was used by HUD-FHA in developing its most current revision of the MPS. In order to maintain consistency with this previous work, the sponsor asked that the same basic approach be used. This has been accomplished to the extent that it matched the objectives of the ongoing DoE-sponsored research. The only parameter that was significantly changed from the previous NBS analysis for the sponsor is the design of the floor to simulate thermal mass on the inside of the structure. This will be explained in greater detail in the following section. Because this study is intended to examine the cost effectiveness of wall insulation, the specification of other shell components was held constant in each climatic location. However, ceiling insulation levels and glazing specifications (e.g., single, double, triple) were adjusted in each location to conform as closely as possible to the proposed MPS. The eight locations to be used in examining the sensitivity of insulation cost effectiveness, the number of long term heating and cooling degree days, and the attic insulation and window glazing specifications used in each location are shown in Table 2. 
Table 2. Cities, Degree Days, Shell Specifications

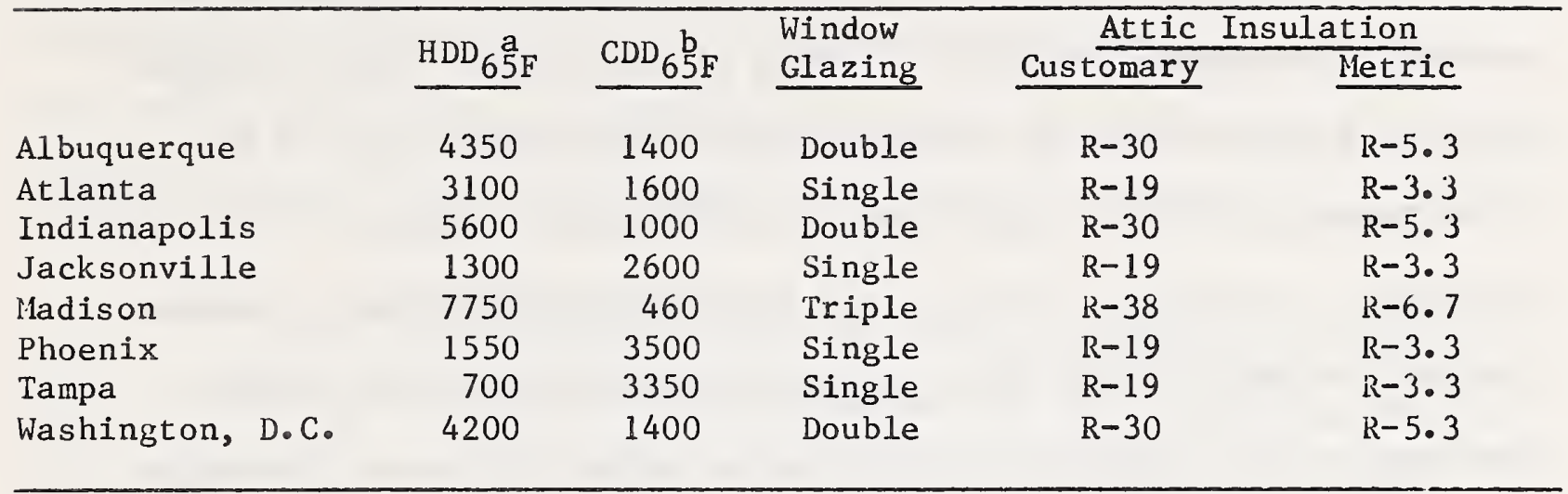

\section{a Heating Degree Days computed at base $65^{\circ} \mathrm{F}$ \\ b Cooling Degree Days computed at base $65^{\circ} \mathrm{F}$}

Note: The base value for "degree days" in SI units has not yet been established, but is expected to be $18^{\circ} \mathrm{C}\left(64.4^{\circ} \mathrm{F}\right)$ rather than $65^{\circ} \mathrm{F}\left(18.33^{\circ} \mathrm{C}\right)$.

Table 3. Prototypical House Parameters ${ }^{a}$

Floor/Attic Area $=1176 \mathrm{ft}^{2}\left(110 \mathrm{~m}^{2}\right)$

Glass Area $=127 \mathrm{ft}^{2}\left[72 \mathrm{ft}^{2}\right.$ south, 55 north $]\left(11.8 \mathrm{~m}^{2}\left[6.7 \mathrm{~m}^{2}, 5.1 \mathrm{~m}^{2}\right]\right)$

Outside Dimensions $=28 \mathrm{ft} \times 42 \mathrm{ft}(8.5 \mathrm{~m} \mathrm{f} 12.8 \mathrm{~m})$
Wall Area - Gross $=1120 \mathrm{ft}^{2}$, Net $=973 \mathrm{ft}^{2}\left(104 \mathrm{~m}^{2}, 90 \mathrm{~m}^{2}\right)$

Door $=20 \mathrm{ft}^{2}\left(1.9 \mathrm{~m}^{2}\right)$

Solar Absorptivity of exterior walls $\mathrm{s}^{\mathrm{b}}=0.50$

Floor weight $=20,0001 \mathrm{bs}(9070 \mathrm{~kg})$

a Based on ranch house design by S. R. Hastings in [1].

b Solar absorptivity varies by color: Very light $=.25$, Light $=.35$, Medium $=.55$, Dark $=.75$, Very dark $=.90$. (Plain concrete block $=$ approx. .55.) 


\subsection{MODELING THE WALLS FOR THERMAL ANALYSIS}

Table 3 provides the basic measurements of the 1176 square foot $\left(110 \mathrm{~m}^{2}\right)$, one-story ranch house used in the simulation of heating and cooling requirements typical of a house of this style. This prototype house is modeled after the "compact" ranch style house proposed by S. R. Hastings [1]* for use in energy conservation research. The house is modeled as one big room as it has been determined through previous analysis at NBS [2] that this more closely simulates actual thermal performance than modeling uncoupled rooms within the house. (Coupling of rooms has not yet been properly simulated). The floor is modeled as a concrete slab weighing $20,0001 \mathrm{bs}(9070 \mathrm{~kg})$ and is essentially adiabatic** (i.e., does not transmit heat into the ground.) This concrete floor is meant to represent the internal mass of the house, including partition walls, fixtures, and furnishings, rather than more typical slab on grade.

The base house is oriented with the long walls facing north and south. of the 127 square feet $\left(11.8 \mathrm{~m}^{2}\right)$ of glass area, 72 square feet $\left(6.7 \mathrm{~m}^{2}\right)$ face south and 55 square feet $\left(5.1 \mathrm{~m}^{2}\right)$ face north. (The glass to gross wall area ratio is approximately 11 percent.) In addition, in three locations the masonry house is turned $90^{\circ}$, with the larger glass area facing west, in order to determine the impact of orientation on the energy savings from insulating the walls. All glass area is assumed to be 50 percent shaded between May and September. The direct solar heat gain is modeled

\footnotetext{
* Numbers in brackets pertain to references listed on p. 49.

$* *$ This is accomplished by modeling a very high thermal resistance beneath the slab in order to isolate it from the ground temperature.
} 
so that 90 percent is distributed directly to the floor, with the remaining 10 percent evenly distributed to the other interior surfaces. (This solar distribution in effect simulates the solar gain of interior partitions and furniture rather than of the inside of the exterior surfaces of the room.)

Operational assumptions are shown in Table 4. Note that a night setback from 68 to $60^{\circ} \mathrm{F}\left(20\right.$ to $\left.15.5^{\circ} \mathrm{C}\right)$ is assumed during the heating season. This night setback, together with the internal mass and south facing windows gives the house some advantage from a "passive" solar aspect.

NBSLD $[2,3]$, the NBS Load Determination program, was used to simulate the heating and cooling loads of the prototype house on an hour-byhour basis for an entire year. Test reference year tapes were used to provide hourly climate data for the locations considered, consistent with the data requirement of NBSLD. Because NBSLD can calculate the thermal response of the walls to a relatively high degree of accuracy, this program is well suited for the thermal analysis in this report. In addition, it has been successfully validated in a number of studies at NBS and elsewhere $[4,5,6]$.

In calculating the thermal performance of the $8^{\prime \prime}(200 \mathrm{~mm})$ block wall, the core section of the block is modeled separately from the web section. NBS analysis has shown that this approach is superior to modeling the wall as homogeneous in mass and thermal resistance. This latter approach is faster in terms of computational effort but exaggerates the thermal response characteristics of the wall. Similarly, wood-frame walls were modeled with studs considered separately from cavities. 
Table 4. Operational Profile of House

Thermos tat settings: ${ }^{a}$

Heating Periods $68^{\circ} \mathrm{F}$ day $\left(20{ }^{\circ} \mathrm{C}\right)$

$60^{\circ} \mathrm{F}$ night $\left(11 \mathrm{p} \cdot \mathrm{m}_{\bullet}-7 \mathrm{a} \cdot \mathrm{m}_{\bullet}\right) \quad\left(15.5^{\circ} \mathrm{C}\right)$

Cooling Periods $78^{\circ} \mathrm{F}$

$\left(25.5^{\circ} \mathrm{C}\right)$

Relative Humidity: $b$

Lower Bound 20\%

Upper Bound $60 \%$

Occupants: 2 adults, 2 children

a No cooling or heating is required when indoor temperature is between heating and cooling thermostat settings.

b Humidification is assumed if the relative humidity falls below 20 percent. No dehumidification is assumed if the air conditioner is not operating; thus the 60 percent upper bound only applies during periods when the air conditioner is operating. 
NBSLD was used to perform extensive simulations of the effects of insulation in an $8^{\prime \prime}(200 \mathrm{~mm})$ masonry block wall and a conventional woodframe wal1. Washington, D. C., which has annual heating and cooling requirements that are near average for the U.S., was used in these simulations to demonstrate certain effects of various insulation levels that can be used to simplify the requirements for simulations in other climates. In particular, the validity of using U-values to linearly interpolate between annual heating and cooling requirements, as calculated by NBSLD analysis, in order to estimate the heating and/or cooling requirements of alternative insulation levels in the same wall type, was demonstrated. Because the mass of alternative insulation types is insignificant relative to the mass of the wall itself, the results of the NBSLD analysis can be used, within reason, for estimating the effects of any type of insulating material practical for the basic wall constructions examined.

Tables $5 A$ and $B$ contain the data points developed through NBSLD analysis of thermal insulation in the furring space of $8^{\prime \prime}$ (200 mm) block walls, with and without perlite insulation in the cores of the blocks, respectively. In addition, data points from the analysis of fiberglass insulation in a wood-frame wall are shown in Table 5C. Table 5D contains the data points for the $90^{\circ}$ rotation of the $8^{\prime \prime}(200 \mathrm{~mm})$ block house in Madison, Phoenix, and Washington, D.C. It should be noted that the U-values in Tables 5A, $B$, and $D$ do not correspond exactly to the HUD-FHA values shown in Table 1 because the former are based on more generalized material specifications.

Total cooling requirements $(C)$ in this report have been divided into two parts: cooling requirements for all hours in which the outdoor dry bulb 
Table $5 \mathrm{~A}$. Wall U-yalues and Annual Heating and Cooling Requirements for $1176 \mathrm{ft}^{2}$ House. ( 8 " Block Walls - no insulation in cores) ${ }^{a}$

Annual Space Heating And Cooling Requirements (million Btu)

\begin{tabular}{|c|c|c|c|c|c|}
\hline & Wall & & & ing & \\
\hline & U-value & Heating & $\mathrm{C}+$ & $\mathrm{C}-$ & $\mathrm{C}$ \\
\hline Albuquerque & $\begin{array}{l}.2417^{b} \\
.1443^{d} \\
.0693^{f}\end{array}$ & $\begin{array}{l}21.89 \\
15.28 \\
10.33\end{array}$ & $\begin{array}{l}9.06 \\
8.72 \\
8.55\end{array}$ & $\begin{array}{l}3.94 \\
4.69 \\
5.61\end{array}$ & $\begin{array}{l}13.00 \\
13.40 \\
14.15\end{array}$ \\
\hline At lanta & $\begin{array}{l}.2417 \\
.1443 \\
.0693\end{array}$ & $\begin{array}{r}16.81 \\
12.55 \\
9.36\end{array}$ & $\begin{array}{l}9.37 \\
9.22 \\
9.18\end{array}$ & $\begin{array}{l}7.43 \\
8.48 \\
9.49\end{array}$ & $\begin{array}{l}16.80 \\
17.70 \\
18.68\end{array}$ \\
\hline Indianapolis & $\begin{array}{l}.2417 \\
.1443 \\
.0693\end{array}$ & $\begin{array}{l}41.34 \\
31.26 \\
23.35\end{array}$ & $\begin{array}{l}6.32 \\
6.18 \\
6.14\end{array}$ & $\begin{array}{l}4.34 \\
5.10 \\
6.04\end{array}$ & $\begin{array}{l}10.65 \\
11.28 \\
12.17\end{array}$ \\
\hline Jacksonville & $\begin{array}{l}.2417 \\
.1443 \\
.0693\end{array}$ & $\begin{array}{l}3.50 \\
2.27 \\
1.47\end{array}$ & $\begin{array}{l}21.66 \\
21.21 \\
20.94\end{array}$ & $\begin{array}{l}11.05 \\
12.01 \\
13.03\end{array}$ & $\begin{array}{l}32.71 \\
33.22 \\
33.97\end{array}$ \\
\hline Madison & $\begin{array}{l}.2417 \\
.1443 \\
.0693\end{array}$ & $\begin{array}{l}48.71 \\
36.23 \\
26.41\end{array}$ & $\begin{array}{l}3.17 \\
3.17 \\
3.21\end{array}$ & $\begin{array}{l}2.20 \\
2.95 \\
3.97\end{array}$ & $\begin{array}{l}5.37 \\
6.12 \\
7.18\end{array}$ \\
\hline Phoenix & $\begin{array}{l}.2417 \\
.1443 \\
.0693\end{array}$ & $\begin{array}{l}3.93 \\
2.56 \\
1.68\end{array}$ & $\begin{array}{l}36.44 \\
33.84 \\
31.86\end{array}$ & $\begin{array}{l}5.57 \\
6.30 \\
7.09\end{array}$ & $\begin{array}{l}42.01 \\
40.14 \\
38.96\end{array}$ \\
\hline Tampa & $\begin{array}{r}.2417 \\
.1443 \\
.0693\end{array}$ & $\begin{array}{l}0.824 \\
0.474 \\
0.275\end{array}$ & $\begin{array}{l}24.26 \\
23.79 \\
23.51\end{array}$ & $\begin{array}{l}13.91 \\
15.15 \\
16.29\end{array}$ & $\begin{array}{l}38.16 \\
38.94 \\
39.80\end{array}$ \\
\hline Washington, D.C. & $\begin{array}{l}.2417 \\
.1618^{c} \\
.1443 \\
.0936^{\mathrm{e}} \\
.0693\end{array}$ & $\begin{array}{r}21.53 \\
16.10 \\
14.92 \\
11.55 \\
9.96\end{array}$ & $\begin{array}{r}10.70 \\
10.34 \\
10.26 \\
10.06 \\
9.98\end{array}$ & $\begin{array}{l}4.97 \\
5.53 \\
5.68 \\
6.20 \\
6.52\end{array}$ & $\begin{array}{l}15.67 \\
15.86 \\
15.94 \\
16.27 \\
16.49\end{array}$ \\
\hline
\end{tabular}

\footnotetext{
a Wall weight = approximately $38 \mathrm{lbs} / \mathrm{ft}^{2}$.

b $\mathrm{U}=.2417$ corresponds to an $8^{\prime \prime}$ block wall with $3 / 4^{\prime \prime}$ nonreflective airspace $(R-0.96)$ and $1 / 2^{\prime \prime}$ gypsum board.
c $U=.1618$, same as above with $R-3$ insulation in place of non- reflective airspace.

d $U=.1443$, same as above with $\mathrm{R}-3.75$ insulation.

e $U=.0936$, same as above with $R-7.5$ insulation.

f $U=.0693$, same as above with R-11.25 insulation.
} 
Table 5A-M. Wall U-values and Annual Heating and Cooling Requirements for $110 \mathrm{~m}^{2}$ House. (200 mm Block Walls - no insulation in cores) ${ }^{a}$

Annual Space Heating And Cooling Requirements (GJ)

Wall

U-value

Albuquerque
Atlanta
Indianapolis

$1.37^{b}$
$0.82^{d}$
$0.39^{f}$

1.37

0.82

0.39
Heating

23.10
15.07
10.90

17.74

13.24

9.88

$$
\begin{aligned}
& 1.37 \\
& 0.82 \\
& 0.39
\end{aligned}
$$

Jacksonville

$$
\begin{aligned}
& 1.37 \\
& 0.82 \\
& 0.39
\end{aligned}
$$

Madison

$$
\begin{aligned}
& 1.37 \\
& 0.82 \\
& 0.39
\end{aligned}
$$

Phoenix

$$
\begin{aligned}
& 1.37 \\
& 0.82 \\
& 0.39
\end{aligned}
$$

Tampa

$$
\begin{aligned}
& 1.37 \\
& 0.82 \\
& 0.39
\end{aligned}
$$

Washington D.C.
43.62

32.98

24.64

3.69

2.39

1.55

50.82

38.22

27.86

\subsection{5}

2.70

1.77

0.87

0.50

0.29

\subsection{2}

16.99

15.74

12.19

10.51

$\frac{\text { Cooling }}{\mathrm{C}+\mathrm{C}-\mathrm{C}}$

\subsection{6}

9.20

9.02

4.16

13.72

$4.95 \quad 14.14$

$5.92 \quad 14.93$

9.89

$7.84 \quad 17.73$

9.73

$8.95 \quad 18.67$

8.63

$8.96 \quad 18.65$
$0.92^{\mathrm{C}}$

0.82

$0.53^{\mathrm{e}}$

0.39
6.67
6.52
6.48

4.58

5.38

6.37

11.24

22.85

22.38

22.09

11.90

12.84

\subsection{4}

3.34

3. 39

38.45

35.70

33.61

25.60

25.10

24.80

11.29

10.91

10.82

10.61

10.53
11.66

12.67

13.75

2. 32

3.11

4.19

34.51

35.05

35.84

\subsection{8}

6.65

7.48

5.67

6.46

7.58

44.32

42.35

41.11

$14.68 \quad 40.26$

$15.98 \quad 41.08$

$17.19 \quad 41.99$

$5.24 \quad 16.53$

$5.83 \quad 16.73$

$5.99 \quad 16.82$

$6.54 \quad 17.17$

$6.88 \quad 17.40$

a Wall weight = approximately $185 \mathrm{~kg} / \mathrm{m}^{2}$.

b U-metric $=1.37$ corresponds to a $200 \mathrm{~mm}$ block wall with $19 \mathrm{~mm}$ non-reflective airspace (R-metric 0.17) and $13 \mathrm{~mm}$ gypsum board.

c $\mathrm{U}$-metric $=0.92$, same as above with R-metric 0.53 insulation in place of non-reflective airspace.

d $\mathrm{U}$-metric $=0.82$, same as above with R-metric 0.66 insulation.

e $U$-metric $=0.53$, same as above with $\mathrm{R}$-metric 1.32 insulation.

f $\mathrm{U}$-metric $=0.39$, same as above with R-metric 1.98 insulation. 
Table 5B. Wall U-values and Annual Heating and Cooling Requirements for $1176 \mathrm{ft}^{2}$ House ( $8^{\circ}$ Block Walls - perlite insulation in cores) ${ }^{a}$

Annual Space Heating and Cooling Requirements (million Btu)

\begin{tabular}{|c|c|c|c|c|c|}
\hline & \multirow[b]{2}{*}{ U-value } & \multirow[b]{2}{*}{ Heating } & \multicolumn{3}{|c|}{ Cooling } \\
\hline & & & $\mathrm{Ct}$ & $\mathrm{C}-$ & $\mathrm{C}$ \\
\hline Albuquerque & $\begin{array}{l}.1116^{b} \\
.0791^{d} \\
.0471^{f}\end{array}$ & $\begin{array}{r}12.66 \\
10.62 \\
8.71\end{array}$ & $\begin{array}{l}8.18 \\
8.26 \\
8.36\end{array}$ & $\begin{array}{l}4.92 \\
5.39 \\
5.96\end{array}$ & $\begin{array}{l}13.10 \\
13.65 \\
14.32\end{array}$ \\
\hline Atlanta & $\begin{array}{r}.1116 \\
.0791 \\
.0471\end{array}$ & $\begin{array}{r}10.77 \\
9.45 \\
8.24\end{array}$ & $\begin{array}{l}8.79 \\
8.93 \\
9.07\end{array}$ & $\begin{array}{l}8.84 \\
9.34 \\
9.82\end{array}$ & $\begin{array}{l}17.63 \\
18.27 \\
18.89\end{array}$ \\
\hline Indianapolis & $\begin{array}{l}.1116 \\
.0791 \\
.0471\end{array}$ & $\begin{array}{l}27.66 \\
24.22 \\
20.88\end{array}$ & $\begin{array}{l}5.89 \\
5.95 \\
6.03\end{array}$ & $\begin{array}{l}5.35 \\
5.81 \\
6.33\end{array}$ & $\begin{array}{l}11.24 \\
11.76 \\
12.36\end{array}$ \\
\hline Jacksonville & $\begin{array}{r}.1116 \\
.0791 \\
.0471\end{array}$ & $\begin{array}{l}1.60 \\
1.35 \\
1.15\end{array}$ & $\begin{array}{l}20.43 \\
20.59 \\
20.71\end{array}$ & $\begin{array}{l}12.40 \\
12.86 \\
13.37\end{array}$ & $\begin{array}{l}32.83 \\
33.45 \\
34.08\end{array}$ \\
\hline Madison & $\begin{array}{l}.1116 \\
.0791 \\
.0471\end{array}$ & $\begin{array}{l}31.72 \\
27.48 \\
23.37\end{array}$ & $\begin{array}{l}2.98 \\
3.06 \\
3.15\end{array}$ & $\begin{array}{l}3.05 \\
3.61 \\
4.29\end{array}$ & $\begin{array}{l}6.03 \\
6.67 \\
7.44\end{array}$ \\
\hline Phoenix & $\begin{array}{l}.1116 \\
.0791 \\
.0471\end{array}$ & $\begin{array}{l}1.74 \\
1.49 \\
1.31\end{array}$ & $\begin{array}{l}32.13 \\
31.60 \\
31.05\end{array}$ & $\begin{array}{l}6.31 \\
6.85 \\
7.37\end{array}$ & $\begin{array}{l}38.44 \\
38.45 \\
38.42\end{array}$ \\
\hline Tampa & $\begin{array}{l}.1116 \\
.0791 \\
.0471\end{array}$ & $\begin{array}{l}0.295 \\
0.233 \\
0.193\end{array}$ & $\begin{array}{l}23.01 \\
23.15 \\
23.27\end{array}$ & $\begin{array}{l}15.68 \\
16.20 \\
16.70\end{array}$ & $\begin{array}{l}38.69 \\
39.35 \\
39.97\end{array}$ \\
\hline Washington, D.C. & $\begin{array}{l}.1116 \\
.0843^{\mathrm{c}} \\
.0791 \\
.0486^{\mathrm{e}} \\
.0471\end{array}$ & $\begin{array}{r}12.56 \\
10.82 \\
10.41 \\
9.12 \\
8.42\end{array}$ & $\begin{array}{l}9.81 \\
9.78 \\
9.78 \\
9.78 \\
9.77\end{array}$ & $\begin{array}{l}6.00 \\
6.33 \\
6.41 \\
6.69 \\
6.87\end{array}$ & $\begin{array}{l}15.81 \\
16.11 \\
16.18 \\
16.47 \\
16.64\end{array}$ \\
\hline
\end{tabular}

a Wall Weight = approximately $401 \mathrm{bs} / \mathrm{ft}^{2}$.

b $U=.1116$ corresponds to an $8 "$ block wall with poured perlite in cores and a non-reflective $3 / 4^{\prime \prime}$ airspace $(R-0.96)$ on inside wall and $1 / 2 "$ gypsum wallboard.

$c U=.853$, same as above with $\mathrm{R}-3$ insulation in place of nonreflective airspace.

$\mathrm{d}^{\mathrm{U}}=.0791$, same as above with $\mathrm{R}-3.75$ insulation.

e $U=.0586$, same as above with $R-7.5$ insulation.

f $U=.0471$, same as above with $R-11.25$ insulation. 
Table 5B-M. Wall U-values and Annual Heating and Cooling Requirements

for $110 \mathrm{~m}^{2}$ House ( $200 \mathrm{~mm}$ Block Walls - perlite insulation in cores) ${ }^{a}$

\section{Annual Space Heating and Cooling Requirements (GJ)}

Wal 1

U-value

Albuquerque

$0.63^{b}$
$0.45^{d}$
$0.27^{f}$

At lanta

0.63

0.45

0.27

Ind i anapolis

0.63

0.45

0.27

Jacksonville

0.63
0.45
0.27

Madison

0.63

0.45

0.27

Phoenix

$$
\begin{aligned}
& 0.63 \\
& 0.45 \\
& 0.27
\end{aligned}
$$

Tampa

$$
\begin{aligned}
& 0.63 \\
& 0.45 \\
& 0.27
\end{aligned}
$$

Washington D.C. 0.63

$0.48^{c}$

0.45

$0.33^{\mathrm{e}}$

0.27
Heating

13.36
11.20
9.19

11.36

9.97

8.69

29.18

25.55

22.03

1.69

1.42

1.21

33.47

28.99

24.66

1.84

1.57

1.38

0.31

0.25

0.20

13.25
11.42
10.98
9.62
8.88

Cooling

$\frac{\text { Cooling }}{\mathrm{C}+} \frac{\mathrm{C}-}{\mathrm{C}-}$

8.63

5.19

13.82

8.71

$5.69 \quad 14.40$

8.82

6.29

15.11

9.27

9.33

18.60

9.42

9.85

19.28

9.57

10.36

19.93

6.21

5.64

11.86

6.28

$6.13 \quad 12.41$

6.36

6.68

13.04

21.55

13.08

34.64

21.72

13.57

35.29

21.85

14.11

35.96

\subsection{4}

3.23

3.32

3.22

6.36

3.81

7.04

4.53

7.85

33.90

33.34

32.76

6.66

40.56

7.23

40.57

7.78

40.54

24.28

24.42

24.55

16.54

40.82

$17.09 \quad 41.52$

$17.62 \quad 42.17$

10.35

10.32

10.32

10.32

10.31
6.33

16.68

$6.68 \quad 17.00$

$6.76 \quad 17.07$

$7.06 \quad 17.38$

$7.25 \quad 17.56$

a Wall weight = approximately $195 \mathrm{~kg} / \mathrm{m}^{2}$.

b $U$-metric $=0.63$ corresponds to a $200 \mathrm{~mm}$ block wall with poured perlite in cores and a non-reflective $19 \mathrm{~mm}$ airspace (R-metric 0.17) on inside wall and $13 \mathrm{~mm}$ gypsum wallboard.

C $\mathrm{U}$-metric $=0.48$, same as above with R-metric 0.53 insulation in place of non-reflective airspace.

d $U$-metric $=0.45$, same as above with R-metric 0.66 insulation.

e $U$-metric $=0.33$, same as above with R-metric 1.32 insulation.

f U-metric $=0.27$, same as above with R-metric 1.98 insulation. 
Table $5 \mathrm{C}$ Wall U-values and Annual Heating and Cooling Requirements for $1176 \mathrm{ft}^{2}$ House (Wood-frame Walls) ${ }^{a}$

Annual Space Heating and Cooling Requirements (million Btu)

Wall

U-values

Al buquerque

$$
\begin{aligned}
& .2130^{b} \\
& .0781^{c} \\
& .0543^{d}
\end{aligned}
$$

At lanta

Indianapolis

.2130

Jacksonville $\quad .2130$

.0781

.0543

Madison

Phoenix

Tampa

Washington, D.C. .2130

$.1515^{\mathrm{C}}$

.0781

$.0710^{\mathrm{e}}$

.0543
.2130

.0781

.0543

.0781

.0543

.2130

.0781

.0543

.2130

.0781

.0543

Heating

$$
\begin{array}{r}
20.52 \\
11.11 \\
9.43
\end{array}
$$

$$
\begin{array}{r}
16.04 \\
9.92 \\
8.81
\end{array}
$$

38.63

24.40

21.81

3.65

1.70

1.38

45.44

27.72

24.51

4.39

2.01

1.61

.2130

.0781
0.862

0.334

19.82

15.64

10.65

10.17

9.04

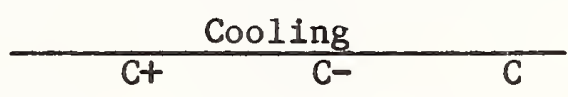

$\begin{array}{rrr}10.10 & 4.42 & 14.52 \\ 9.02 & 5.45 & 14.48 \\ 8.79 & 5.82 & 14.61\end{array}$

$10.33 \quad 7.56 \quad 17.89$

$9.55 \quad 9.15 \quad 18.69$

$9.39 \quad 9.57 \quad 18.94$

$\begin{array}{lll}6.88 & 4.57 & 11.45 \\ 6.38 & 5.82 & 12.20 \\ 6.29 & 6.20 & 12.49\end{array}$

$\begin{array}{lll}23.12 & 10.72 & 33.84\end{array}$

$21.48 \quad 12.62 \quad 34.10$

$21.18 \quad 13.07 \quad 34.25$

$\begin{array}{lll}3.67 & 2.71 & 6.38 \\ 3.38 & 3.88 & 7.27 \\ 3.32 & 4.25 & 7.57\end{array}$

$\begin{array}{lll}37.56 & 6.06 & 43.62\end{array}$

$\begin{array}{lll}32.80 & 6.87 & 39.67\end{array}$

$\begin{array}{lll}31.86 & 7.17 \quad 39.03\end{array}$

$25.61 \quad 13.42 \quad 39.03$

$24.04 \quad 15.79 \quad 39.83$

$\begin{array}{lll}11.32 & 4.94 & 16.27 \\ 10.88 & 5.37 & 16.25 \\ 10.31 & 6.26 & 16.57 \\ 10.25 & 6.36 & 16.61 \\ 10.10 & 6.64 & 16.73\end{array}$

a Wal1 Weight = approximately $61 \mathrm{bs} / \mathrm{ft}^{2}$.

b $U=.2130$ corresponds to a $2^{\prime \prime} \times 4^{\circ}$ frame wall with 3-1/2" non-reflective airspace $(R-0.96)$.

c $U=.1515$, same as above with reflective airspace $(R-3.0)$.

d $U=.0781$, same as above with $\mathrm{R}-11$ insulation in airspace.

e $U=.0710$, same as above with $R-13$ insulation in airspace.

f $U=.0543$ corresponds to a $2^{\circ} \times 6^{\prime \prime}$ frame wall with nominal R-19 insulation in airspace. 
Table 5C-M. Wall U-values and Annual Heating and Cooling Requirements for $110 \mathrm{~m}^{2}$ House (Wood-frame Walls)

\section{Annual Space Heating and Cooling Requirements (GJ)}

Wa11

U-value

Albuquerque

At lanta

Jacksonville

1.21

0.44

0.31

Madison

1.21

0.44

0.31

Phoenix

1.21

0.44

0.31

Tampa

1.21

0.44

Washington, D.C.

1.21
$0.86^{c}$
0.44
$0.40^{e}$
0.31

Heating

$$
\begin{array}{r}
21.65 \\
11.72 \\
9.95
\end{array}
$$

$$
\begin{array}{r}
16 \cdot 92 \\
10.47 \\
9.30
\end{array}
$$

\begin{abstract}
40.76
25.74

23.01
\end{abstract}

$$
\begin{aligned}
& 3.85 \\
& 1.79 \\
& 1.46
\end{aligned}
$$

47.94
29.25
25.86

4.63

2.12

1.70

0.91

0.35

20.91

16.50

11.24

10.73

9.54

\begin{tabular}{rcc}
\multicolumn{3}{c}{ Cooling } \\
\hline C+ & C- & C \\
& & \\
10.66 & 4.66 & 15.32 \\
9.52 & 5.75 & 15.28 \\
9.27 & 6.14 & 15.41
\end{tabular}

$\begin{array}{rrr}10.90 & 7.98 & 18.88 \\ 10.08 & 9.65 & 19.72 \\ 9.91 & 10.10 & 19.98\end{array}$

7.26

4.82

12.08

6.73

6.14

12.87

6.64

6.54

13.18

$\begin{array}{lll}24.39 & 11.31 & 35.70 \\ 22.66 & 13.31 & 35.98 \\ 22.35 & 13.79 & 36.14\end{array}$

$\begin{array}{lll}3.87 & 2.86 & 6.73 \\ 3.57 & 4.09 & 7.67 \\ 3.50 & 4.48 & 7.99\end{array}$

39.63

6.39

46.02

34.61

7.25

41.85

33.61

7.56

41.18

$27.02 \quad 14.16 \quad 41.18$

$25.36 \quad 16.66 \quad 42.02$

$\begin{array}{lll}11.94 & 5.21 & 17.17 \\ 11.48 & 5.67 & 17.14 \\ 10.88 & 6.60 & 17.48 \\ 10.81 & 6.71 & 17.52 \\ 10.66 & 7.01 & 17.65\end{array}$

a Wall weight $=$ approximately $30 \mathrm{~kg} / \mathrm{m}^{2}$.

b U-metric $=1.21$ corresponds to a $38 \times 89 \mathrm{~mm}$ frame wall with $89 \mathrm{~mm}$ non-reflective airspace (R-metric 0.17).

c U-metric $=0.86$, same as above with reflective airspace (R-metric 0.53 ).

d U-metric $=0.44$, same as above with R-metric 1.94 insulation in airspace.

e U-metric $=0.40$, same as above with R-metric 2.29 insulation in airspace.

f $\mathrm{U}$-metric $=0.31$, corresponds to a $38 \times 140 \mathrm{~mm}$ frame wall with nominal $\mathrm{R}$-metric 3.35 insulation in airspace. 
Table 5 D. Wall U-values and Annual Heating and Cooling Requirements for $1176 \mathrm{ft}^{2}$ House - 90 Rotation ( $8^{\prime \prime}$ Block Walls - no insulation in cores)

Annual Space Heating and Cooling Requirements (million Btu)

Wall

U-values

Madison

.2417

.1443

.0693

Phoenix

.2417

.1443

.0693

Washington, D.C. .2417

.1443

.0693
Heating

50.988

38.595

28.824

4.647

3.107

2.046

23.081

16.424

11.389
Cooling

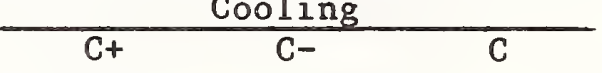

$\begin{array}{lll}3.650 & 2.863 & 6.513 \\ 3.645 & 3.751 & 7.396 \\ 3.685 & 4.863 & 8.548\end{array}$

38.960

6.715

44.675

$\begin{array}{lll}36.393 & 6.163 & 42.556\end{array}$

$\begin{array}{lll}34.436 & 6.664 & 41.100\end{array}$

$\begin{array}{lll}11.793 & 5.869 & 17.662\end{array}$

$\begin{array}{lll}11.363 & 6.651 & 18.014\end{array}$

$\begin{array}{lll}11.094 & 7.456 & 18.550\end{array}$ 
Table 5D-M. Wall U-values and Annual Heating and Cooling Requirements for $110 \mathrm{~m}^{2}$ House - $90^{\circ}$ Rotation (200 mm Block Walls - no insulation in cores)

Annual Space Heating and Cooling Requirements (GJ)

$$
\begin{aligned}
& \text { Wall } \\
& \text { U-value }
\end{aligned}
$$

Madison

$$
1.37
$$

0.82

0.39

Phoenix

$$
\begin{aligned}
& 1.37 \\
& 0.82 \\
& 0.39
\end{aligned}
$$

Washington, D.C. 1.37

0.82

0.39

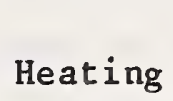

$$
\begin{aligned}
& 53.80 \\
& 40.72 \\
& 30.41
\end{aligned}
$$

$$
\begin{aligned}
& 4.90 \\
& 3.28 \\
& 2.16
\end{aligned}
$$

24.35

17.33

12.02

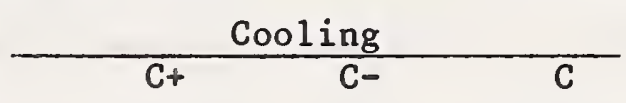

$\begin{array}{lll}3.85 & 3.02 & 6.87 \\ 3.85 & 3.96 & 7.80 \\ 3.89 & 5.13 & 9.02\end{array}$

41.11

38.40

6.03

6.50

7.03

47.13

36.33

12.44

11.99

6.19

7.02

11.70

7.87
44.90

43.36

18.63
19.01
19.57 
temperature is $78^{\circ} \mathrm{F}\left(25.5^{\circ} \mathrm{C}\right)$ or greater $(\mathrm{C}+)$, and cooling requirements for all hours in which the outdoor dry bulb temperature is below $78^{\circ} \mathrm{F}\left(25.5^{\circ} \mathrm{C}\right)$ (C-). (Note that $\mathrm{C}=\mathrm{C}-+\mathrm{C}+$.$) If windows are assumed to be closed at all$ times, $C$ is the best representation of annual cooling requirements. If windows are opened for natural ventilation when the outdoor temperature is below $78^{\circ}\left(25.5^{\circ} \mathrm{C}\right), \mathrm{C}+$ is the best representation. In fact, actual operating practices are usually somewhere between these two extremes. For example, during spring and fall windows and doors may be left open for natural ventilation more frequently than during the summer months. Noise, humidity, air pollution, and security considerations may encourage leaving windows closed more than they might be otherwise, especially in urban areas.

The data points in Tables 5A, 5B, and 5C are shown graphically in Figures 1-8 for the eight cities examined. Visual examination of these figures shows that a change in wall U-value has a very significant effect on heating requirements while having a minimal effect on cooling requirements. In general, the masonry walls perform slightly better than wood-frame walls for the same $U-v a l u e$ in the range of 0.05 to 0.15 (U-metric 0.28 to 0.85 ), which is the range of major interest. The effects of mass in reducing energy requirements appear to be most noticeable with respect to the cooling requirements in hours when the outdoor temperature exceeds the thermostat setpoint $\left(78^{\circ} \mathrm{F}\left(25.5^{\circ} \mathrm{C}\right)\right)$. For cooling hours in which the outdoor temperature is lower than the thermostat setpoint, the wood-frame wall generally (although not in every case) outperforms the masonry wall. The summation of $\mathrm{C}+$ and $\mathrm{C}$ - data generally shows that the masonry walls have a slight (1-5 percent) advantage over wood-frame walls when both are interpolated to a 


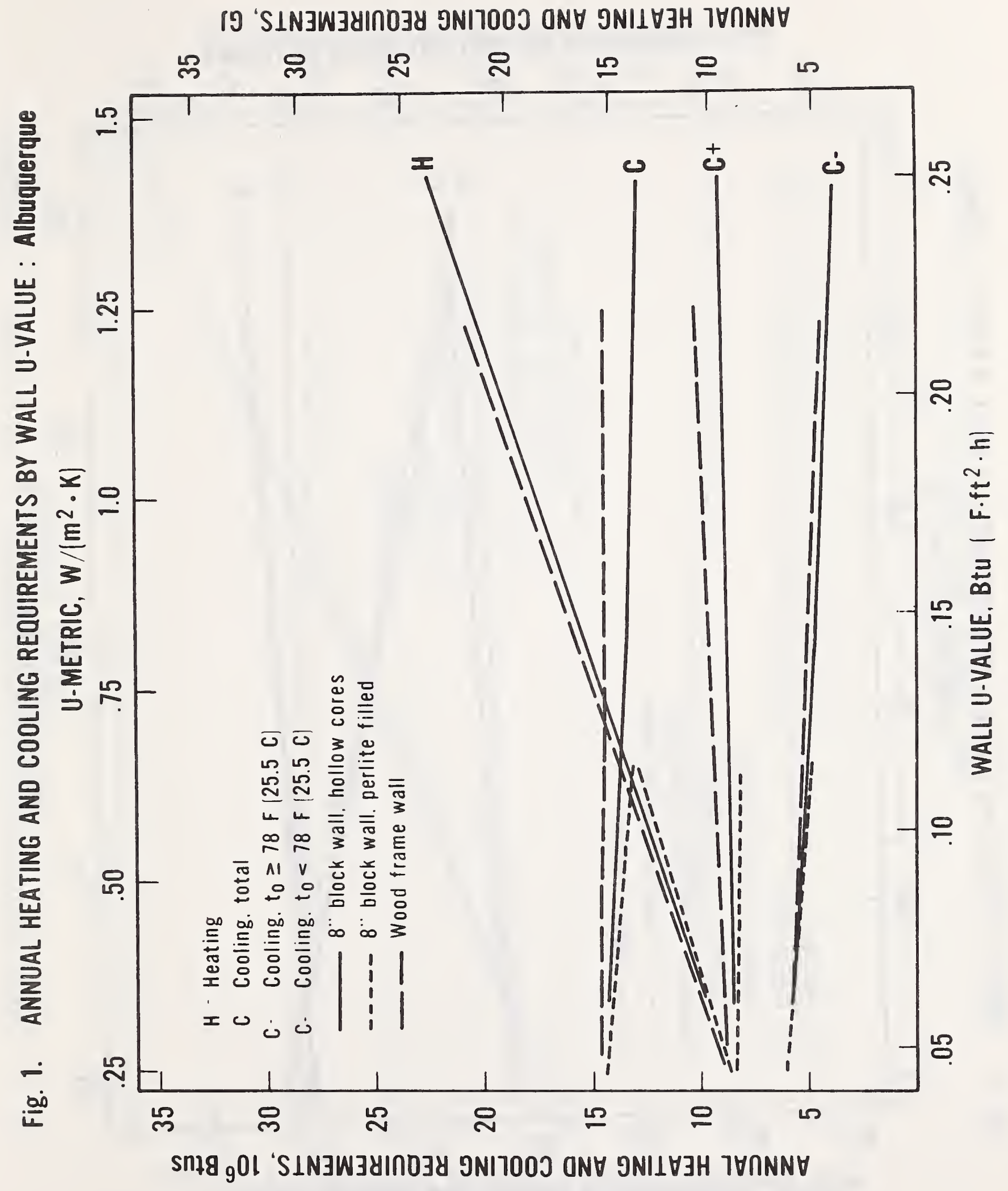




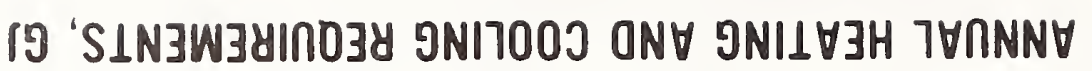

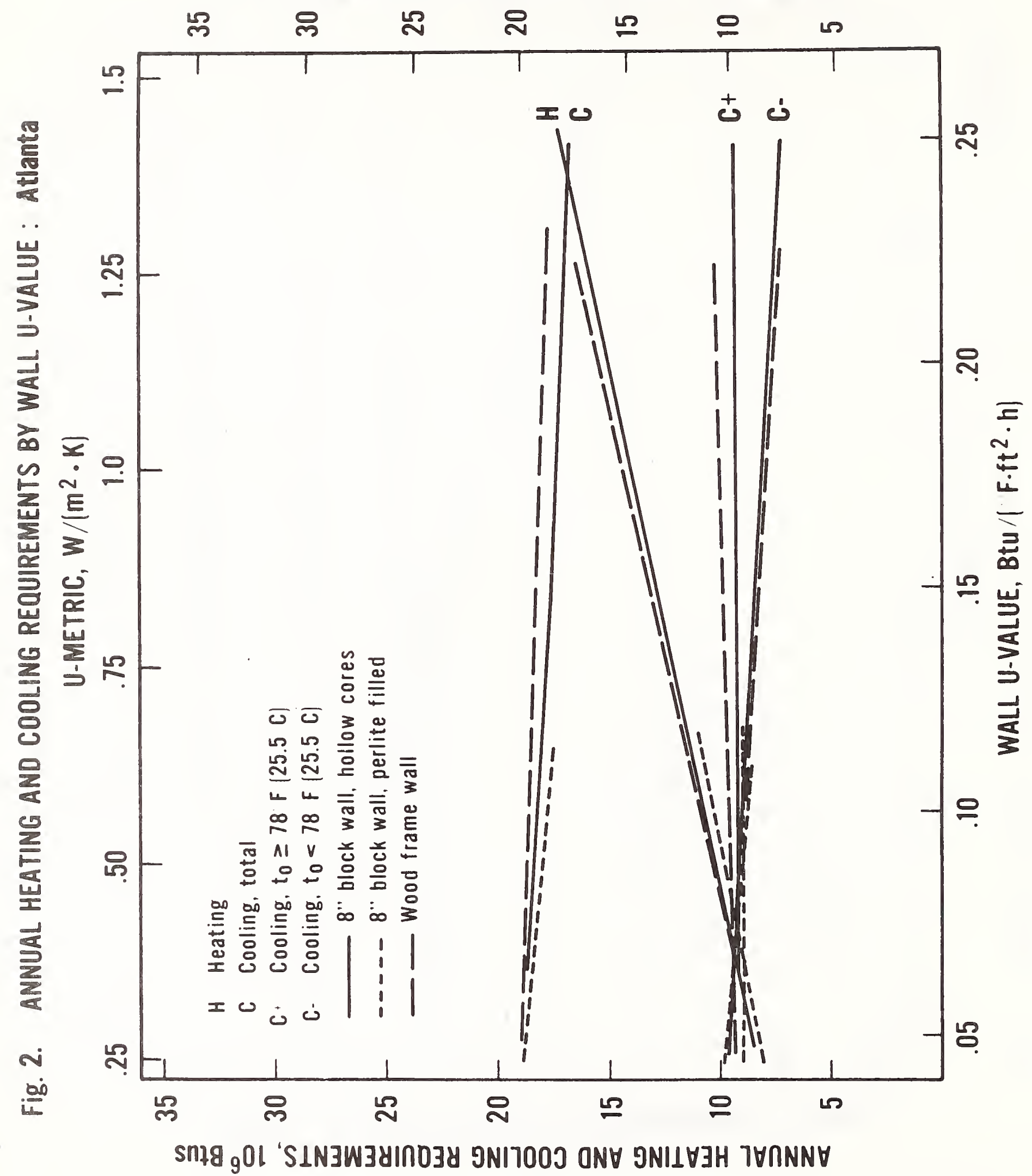




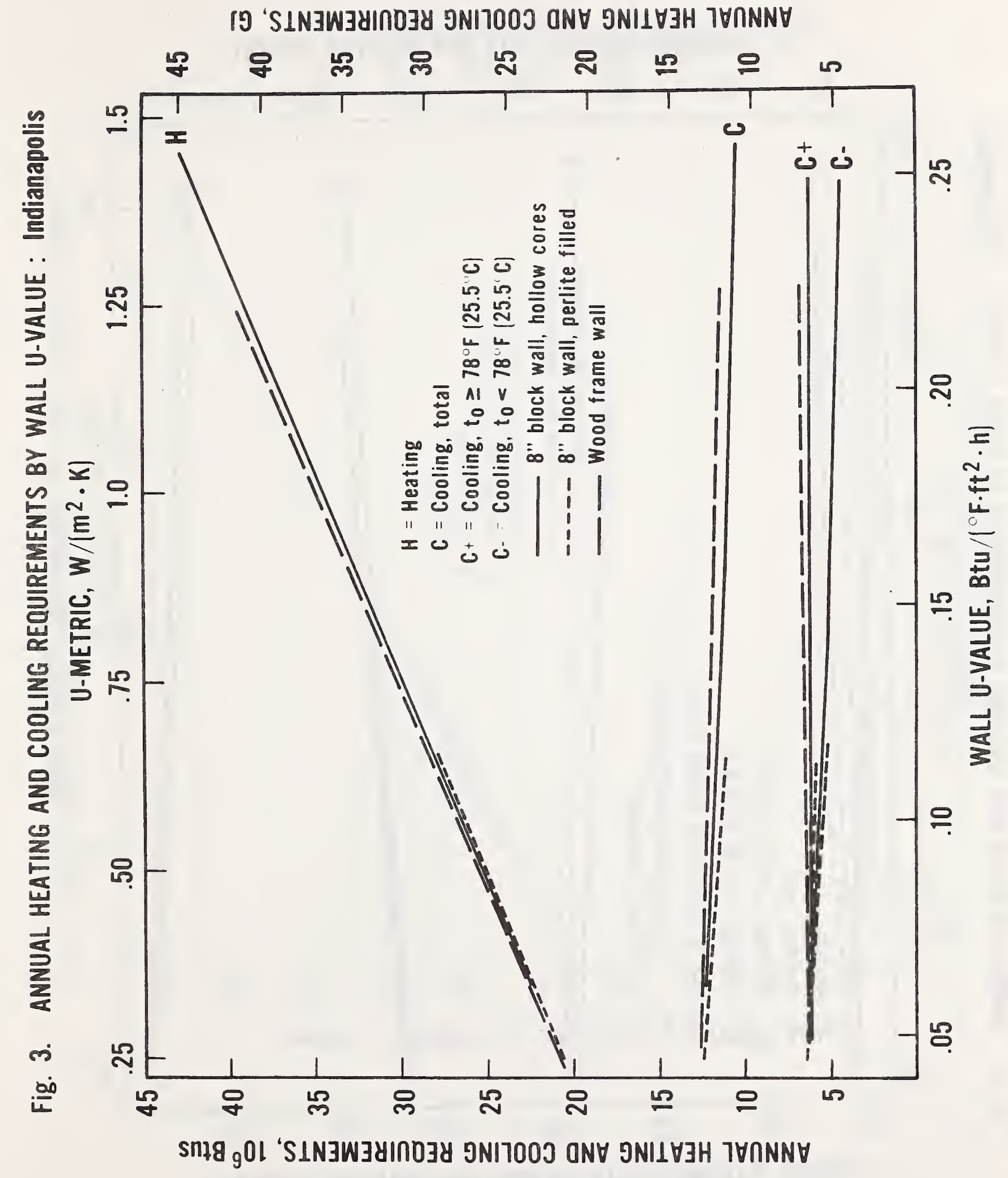




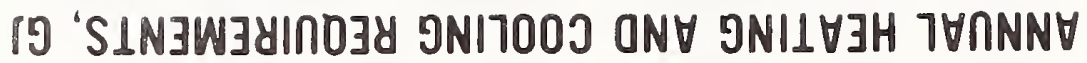

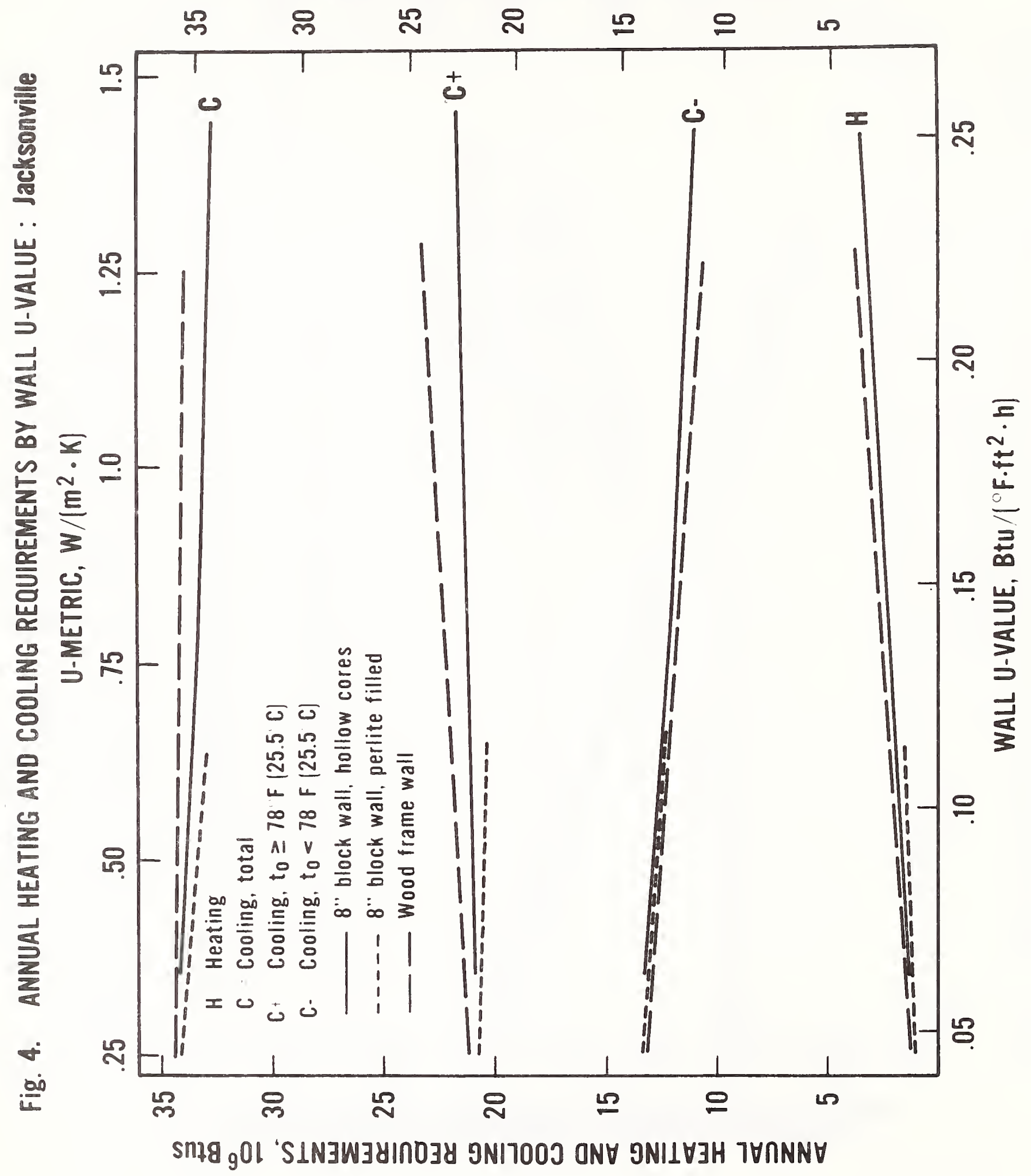




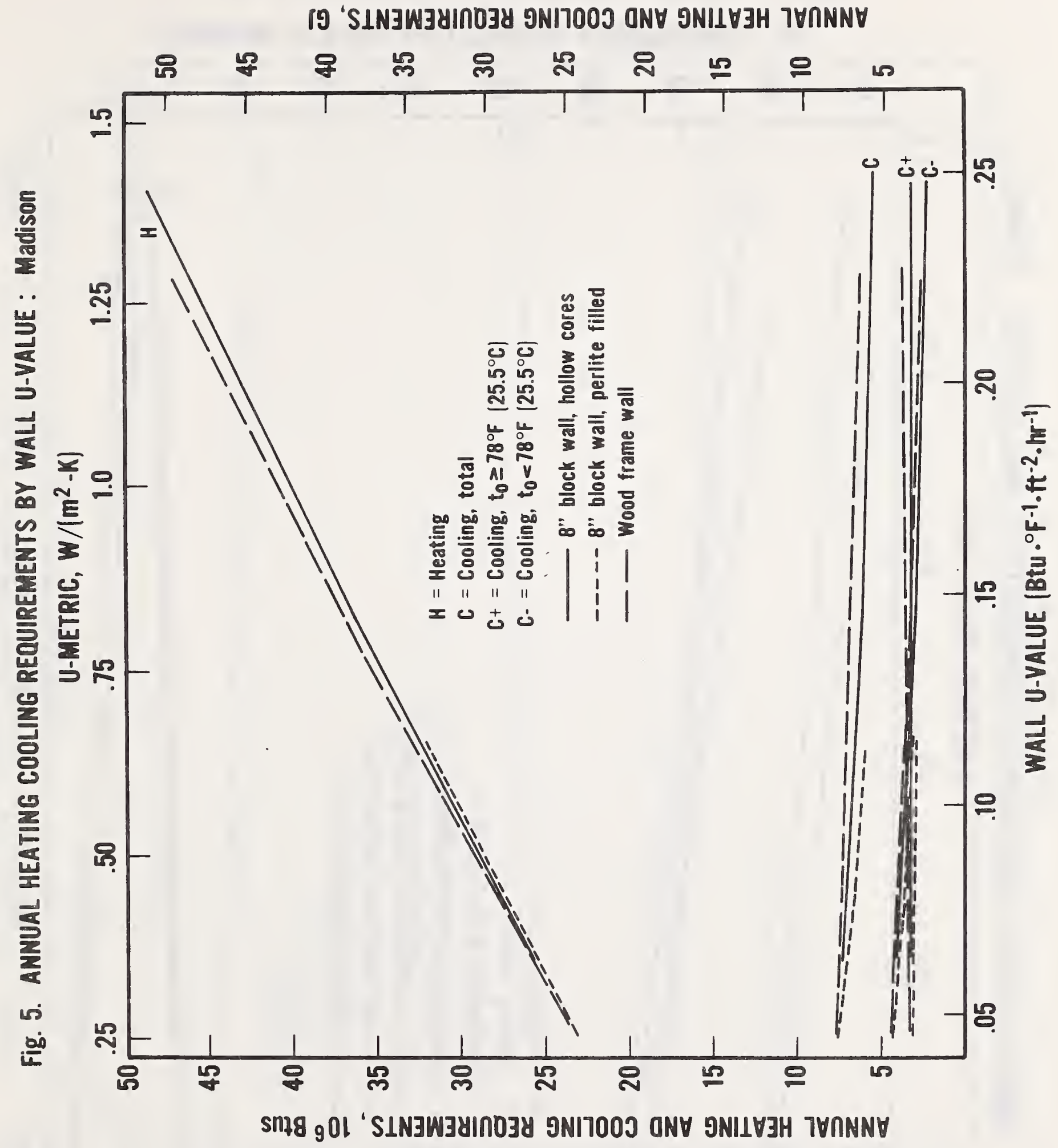




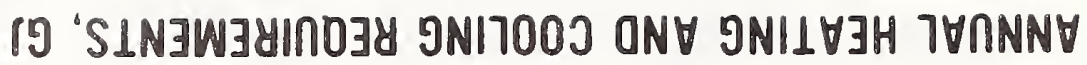

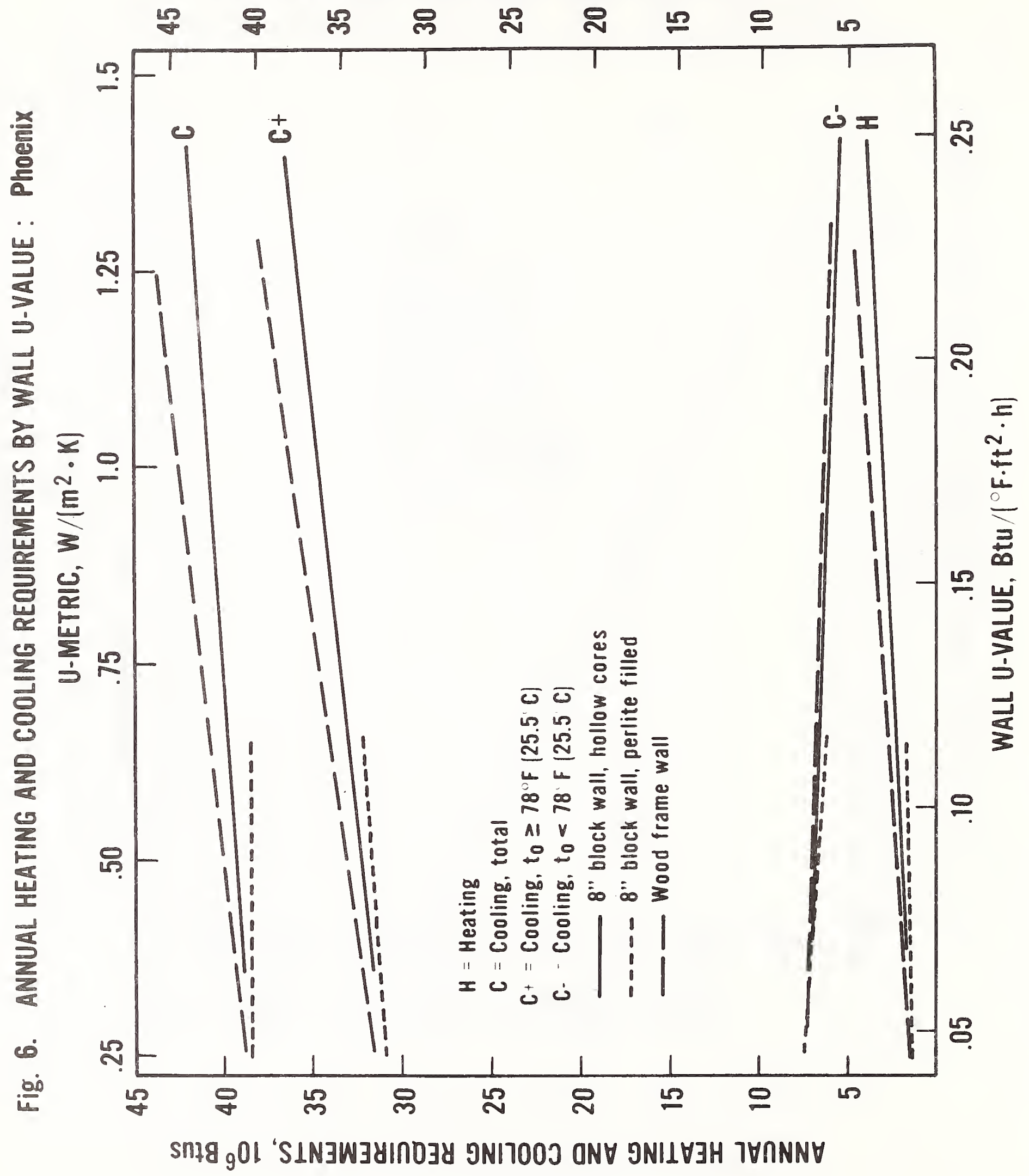




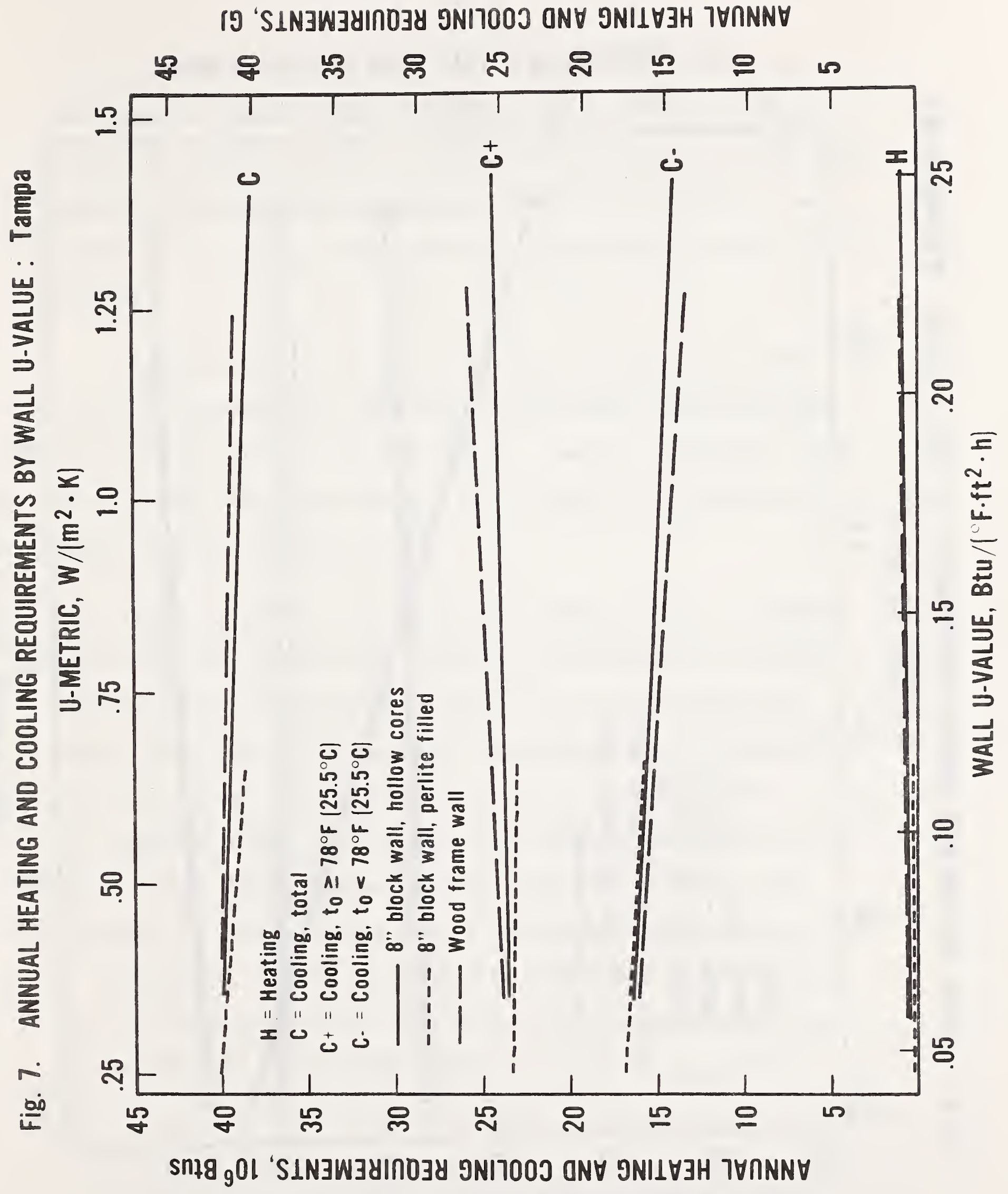




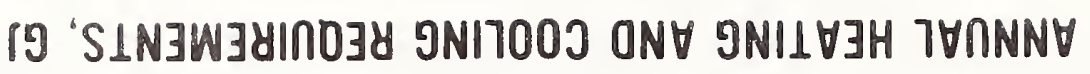

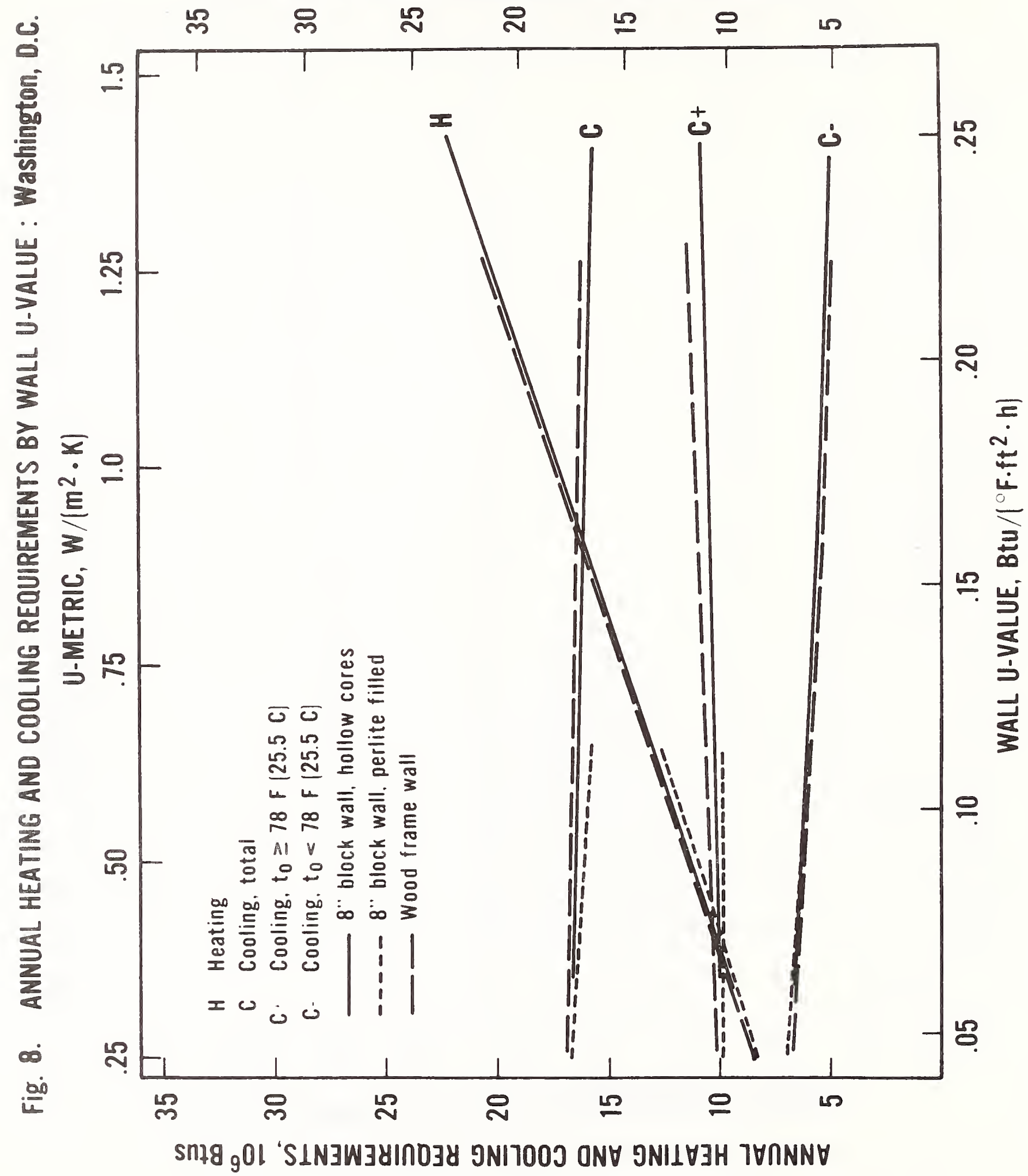


$\mathrm{U}$ of $0.1 \mathrm{Btu} \mathrm{hr} \mathrm{ft}^{-2}{ }^{\circ} \mathrm{F}^{-1}\left(\mathrm{U}\right.$ metric $\left.=0.57 \mathrm{~W} / \mathrm{m}^{2} \cdot{ }^{\circ} \mathrm{K}\right) \cdot$ Comparative performance of masonry and wood-frame walls at this U-value is shown in Table 6. However, it must be stressed that the purpose of this report is to determine the cost-effective level of insulation in each system, not to determine the U-value that provides equal thermal performance.

Comparison of Tables $5 \mathrm{~A}$ and $5 \mathrm{D}$ show that the house rotated $90^{\circ}$ to the west has somewhat higher heating and cooling requirements than the base houses for the same wall design. However, differences in the energy savings due to reductions in wall U-value are not significant enough to warrant further analysis.

Because the effects of insulation on cooling loads are generally quite small, and because these effects vary from negative to positive depending on the way the house is operated, it is recommended that, except in the case of Phoenix, only the heating saving be considered in the final economic analysis of both masonry and wood-frame walls. Since the insulation in the walls of the house in Phoenix has a distinct beneficial effect even with windows always closed, the benefits of wall insulation in reducing annual cooling requirements should be recognized in that climate. A sensitivity analysis is performed to determine the effect of this recommendation on economic levels of insulation. This will be discussed later in this report. 
Table 6. Normalized Annual Heating and Cooling Requirements in million Btu (GJ), Wall $U=0.10^{\mathrm{a}}(\mathrm{U}$-metric $=0.57)$

$$
\begin{gathered}
\text { Wood Frame } \\
6 \mathrm{bs} / \mathrm{ft}^{2}\left(30 \mathrm{~kg} / \mathrm{m}^{2}\right)
\end{gathered} \quad \begin{gathered}
8^{\circ 0}(200 \mathrm{~mm}) \text { Block } \\
381 \mathrm{bs} / \mathrm{ft}^{2}\left(185 \mathrm{~kg} / \mathrm{m}^{2}\right)
\end{gathered} \quad \begin{gathered}
\text { Wood Frame } \\
8^{\prime \prime} \text { Block }
\end{gathered}
$$

Al buquerque

$\begin{array}{llllll}\text { Heating } & 12.64 & (13.34) & 12.36 & (13.04) & 1.023 \\ \text { Cooling } & 14.48 & (15.28) & 13.85 & (14.61) & 1.045\end{array}$

At lanta

$\begin{array}{llllll}\text { Heating } & 10.91 & (11.51) & 10.67 & (11.26) & 1.022 \\ \text { Cooling } & 18.56 & (19.58) & 17.69 & (18.66) & 1.049\end{array}$

Indianapolis

$\begin{array}{llllll}\text { Heating } & 26.71 & (28.18) & 26.55 & (28.01) & 1.006 \\ \text { Cooling } & 12.08 & (12.75) & 11.90 & (12.56) & 1.015\end{array}$

Jacksonville

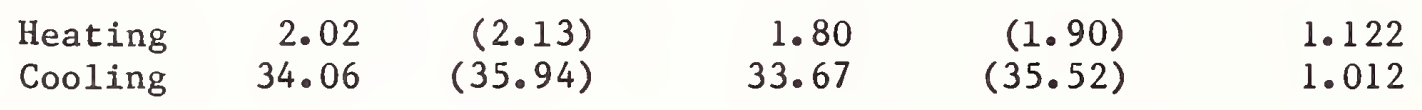

Madison

$\begin{array}{lrrrrr}\text { Heating } & 30.60 & (32.28) & 30.43 & (32.11) & 1.005 \\ \text { Cooling } & 7.12 & (7.51) & 6.75 & (7.12) & 1.055\end{array}$

Phoenix

\begin{tabular}{|c|c|c|c|}
\hline Heating & 2.40 & $\begin{array}{r}(2.53) \\
(42.53)\end{array}$ & 2.04 \\
\hline
\end{tabular}

Tampa

\begin{tabular}{|c|c|c|}
\hline ng & 0.42 & $(0.44)$ \\
\hline Cooling & 39.70 & $(41.89)$ \\
\hline
\end{tabular}

Washington, D.C.

$\begin{array}{llllll}\text { Heating } & 12.14 & (12.81) & 11.98 & (12.64) & 1.013 \\ \text { Cooling } & 16.47 & (17.38) & 16.22 & (17.11) & 1.015\end{array}$

a Interpolated from data in Table $5 \mathrm{~A}$ and $\mathrm{C}$ using nearest two points to $U=0.10(U$-metric $=0.57)$.

b No insulation in cores, $100 \mathrm{pcf}\left(1600 \mathrm{~kg} / \mathrm{m}^{2}\right)$ concrete. 
Because of the generally linear nature of the performance data for each wall type as a function of its U-value, and because the results of the NBSLD analysis are not sensitive to the type of insulation material examined, but rather only to its thermal conductivity and thickness, interpolation between the data points actually examined and any other U-values within this range can be accomplished with considerable confidence. ${ }^{*}$ The data points were generated using non-reflective air spaces, reflective air spaces, and several levels of rigid foam insulation. This approach allows extreme flexibility in analyzing different insulation materials at different thicknesses as long as they are considered for the same wall type and same placement within the wall itself. The polystyrene insulation levels examined for the sponsor are for the most part the same as used in establishing the data points with NBSLD, although minor differences in the wall U-values occur due to minor differences in material specifications. The NBSLD data points are interpolated to provide results which correspond exactly to the HUD-FHA wall specifications. The analysis of the thermal performance of isocyanurate insulation is also accomplished by interpolating the NBSLD data points, using the thermal properties of the isocyanurate insulation provided by HUD-FHA in Table 1.

* For example, using the highest and lowest U-values for $8^{\prime \prime}(200 \mathrm{~mm})$ block walls in Washington, D.C., .2417 and .0693 (U-metric 1.37 and 0.39), interpolation for $U=.1443$ ( $U$-metric $=0.82$ ) results in annual heating and cooling requirements, respectively, of 14.99 and $16.13 \times 10^{6} \mathrm{Btu}(15.82$ and $17.02 \mathrm{GJ})$. NBSLD calculations at $U=.1443(U$-metric $=0.82$ ) result in Annual Heating Requirements and Annual Cooling Requirements of 14.92 and $15.94(15.74$ and $16.82 \mathrm{GJ})$, respectively. In general interpolations are made over a considerably narrower range. 
The effects of the alternative insulation specifications on actual energy savings are analyzed for four heating system types: gas furnace, oil furnace, electric resistance furnace, and heat pump. Seasonal performances assumed for all systems in each location are shown in Table 7. The seasonal performances of combustion heating systems are based on an analysis by Chi, Kelly, and Didion of good quality gas and oil furnaces in a range of climates [7]. In all cases an electric pilot light is assumed. In locations other than Jacksonville, Tampa, and Phoenix, a stack damper is assumed to be installed on gas furnaces. (A stack damper is not likely to be cost effective in climates with small annual heating requirements. Stack dampers for oil furnaces are not commonly available at present.) Note that there is some small variation, by climate, in the seasonal performance of combustion furnaces, consistent with NBS research results. Heat pump seasonal performance data are based on research by Gordian Associates [8]. In most cases interpolations were made from the Gordian data using heating degree-day data for the locations examined.

The seasonal coefficient of performance (COP) for central air conditioners varies little by climate. The COP of 2.3 is approximately equivalent to an SEER (seasonal energy efficiency ratio) of 8.0 , consistent with most manufacturers' middle-of-the-line products.

\subsection{TECHNIQUES OF ECONOMIC ANALYSIS}

In order to establish the cost effectiveness of the alternative insulation levels to be examined, a life-cycle analysis of the insulation costs and savings is performed. That is, the present value of all energy savings over the life of the wall, in dollar terms, due to each improvement 
Table 7. Heating and Cooling System Efficiencies (Seasonal)

\begin{tabular}{|c|c|c|c|c|c|}
\hline & $\begin{array}{c}\text { Gas } \\
\text { Furnace } \\
\end{array}$ & $\begin{array}{c}0 i 1 \\
\text { Furnace } \\
\end{array}$ & $\begin{array}{l}\text { Electric } \\
\text { Furnace } \\
\end{array}$ & $\begin{array}{l}\text { Heat } \\
\text { Pump }\end{array}$ & $\begin{array}{c}\text { Central } \\
\text { Air } \\
\text { Conditioner } \\
\end{array}$ \\
\hline Albuquerque & .76 & .78 & 1.0 & 2.05 & 2.3 \\
\hline Atlanta & .76 & .78 & 1.0 & 2.15 & 2.3 \\
\hline Indianapolis & .76 & .78 & 1.0 & 1.85 & $2 \cdot 3$ \\
\hline Jacksonville & .69 & .78 & 1.0 & 2.30 & 2.3 \\
\hline Madison & .74 & .76 & 1.0 & 1.55 & $2 \cdot 3$ \\
\hline Phoenix & .69 & .78 & 1.0 & 2.20 & 2.3 \\
\hline Tampa & .69 & .78 & 1.0 & $2 \cdot 30$ & 2.3 \\
\hline Washington, D.C. & .74 & .78 & 1.0 & 2.05 & 2.3 \\
\hline
\end{tabular}


in insulation is compared with the cost of that improvement to determine whether or not it is cost justified. Only if the incremental savings of additional levels of insulation, over the next lower level, are greater than or equal to their incremental cost can those additional levels be cost justified on a life-cycle basis.

Consistent with the previous economic analysis of conservation measures performed by NBS for the HUD-FHA, a 30-year life, 10 percent (nominal) discount rate, and regional fuel price projections published by DoE [9] were used. The 30-year life is based on the length of a typical mortgage, which, in effect, testifies to the fact that the expected life of the average house and, by inference, the wall insulation, is at least that long.

Life-cycle savings (LCS) are computed as follows:*

$$
\begin{aligned}
\text { LCS } & =\frac{\Delta A H R}{\eta_{H}} \cdot \operatorname{cost} / \text { unit }_{H} \cdot \text { unit }_{\mathrm{H}} / 10^{6} \mathrm{Btu}{ }^{\mathrm{PWF}} \mathrm{H} \\
& +\frac{\Delta \mathrm{ACR}}{n_{\mathrm{C}}} \cdot \cos \mathrm{t} / \mathrm{kWh} \cdot \mathrm{kwh} / 10^{6} \mathrm{Btu} \cdot \mathrm{PWF}_{\mathrm{C}},
\end{aligned}
$$

where $\triangle \mathrm{AHR}$ is the change in annual heating requirements, in $10^{6} \mathrm{Btu}$, $\triangle A C R$ is the change in annual cooling requirements, in $10^{6} \mathrm{Btu}$,

$n_{H} \quad$ is the seasonal efficiency of the heating system,

${ }^{n} \mathrm{C}$ is the seasonal coefficient of performance of the cooling system, cost/unit ${ }_{H}$ is the current cost per unit (e.g•, therm, gallon, kWh) of heating fuel,

cost/kWh is current cost per kilowatt hour, unit $\mathrm{H}_{\mathrm{H}} / 10^{6} \mathrm{Btu}$ is the number of heating fuel units per million Btu (e.g., therm $=10$, gallon of oil $=7.14, \mathrm{kWh}=293$ ), and

$\mathrm{PWH}_{\mathrm{H}}$ and $\mathrm{PWF}_{\mathrm{C}}$ are the present worth factors for the heating fuel and $\mathrm{kWh}$, respectively.

* For metric equivalent, use GJ instead of $10^{6} \mathrm{Btu}$. 
DoE projections for regional energy prices are based on the "Trendlong" series with an assumption of 5 percent increase in oil constant dollar costs each year. Energy costs are shown in Table 8.

The present worth factor (PWF) is critical to the life-cycle-cost analysis because it converts first-year dollar savings to present-value, life-cycle savings. The PWF is a function of the life (30 years), discount rate (10 percent) and projected rate of fuel price increases. Since the projected rate of fuel price increases published by DoE vary by fuel type and region, different PWF are used for each city and heating fuel used. The PWF for each city and fuel type used in the economic analysis are shown in Table 9.

Insulation costs for masonry walls were based on the 1978 Means' Building Construction Cost Data [10] where practical. The material cost of isocyanurate was taken from the 1978 "Masonry Cost Guide" [11], but the insulation cost and overhead and profit factors are the same as used for polystyrene in Means. The costs for $R-11$ and $R-13$ ( $R$-metric 1.9 and 2.3) fiberglass insulation in a nominal $2^{\circ} \times 4^{\prime \prime}(38 \times 89 \mathrm{~mm})$ wood-frame house were based on NAHB Research Foundation data provided under contract to NBS. These costs are slightly lower than Means but are probably more typical of current residential construction. The cost of the polystyrene sheathing is based on the additional material cost net of the cost of normal asphalt impregnated sheathing, with no additional installation cost, since the former essentially substitutes for the latter. All cost data are shown in Table 10. In order to determine the sensitivity of the results to installed insulation costs, a second analysis with costs increased by 25 percent was made. 
Table 8. Energy Costs ${ }^{a}-1978$ Dollars

\begin{tabular}{|c|c|c|}
\hline $\begin{array}{c}\text { Gas } \\
\text { (Therm) }\end{array}$ & $\begin{array}{c}0 i 1 \\
\text { (Gallon) } \\
\end{array}$ & $\begin{array}{c}\text { Electric } \\
(\mathrm{kWh})\end{array}$ \\
\hline .180 & .470 & .035 \\
\hline .210 & .490 & .035 \\
\hline .220 & .470 & .040 \\
\hline .210 & .490 & .035 \\
\hline .220 & .470 & .040 \\
\hline .220 & .510 & .042 \\
\hline .210 & .490 & .035 \\
\hline .270 & .500 & .046 \\
\hline
\end{tabular}

a Based on [9], Trendlong Scenario with $5 \%$ annual increase in real oil prices. These are regional energy prices, not specific to the city indicated.

b No metric equivalents are shown. In SI units, all energy costs can be compared in terms of $\$ / \mathrm{MJ}$.

Table 9. Present Worth Factors ${ }^{\text {a }}$ for Energy Savings

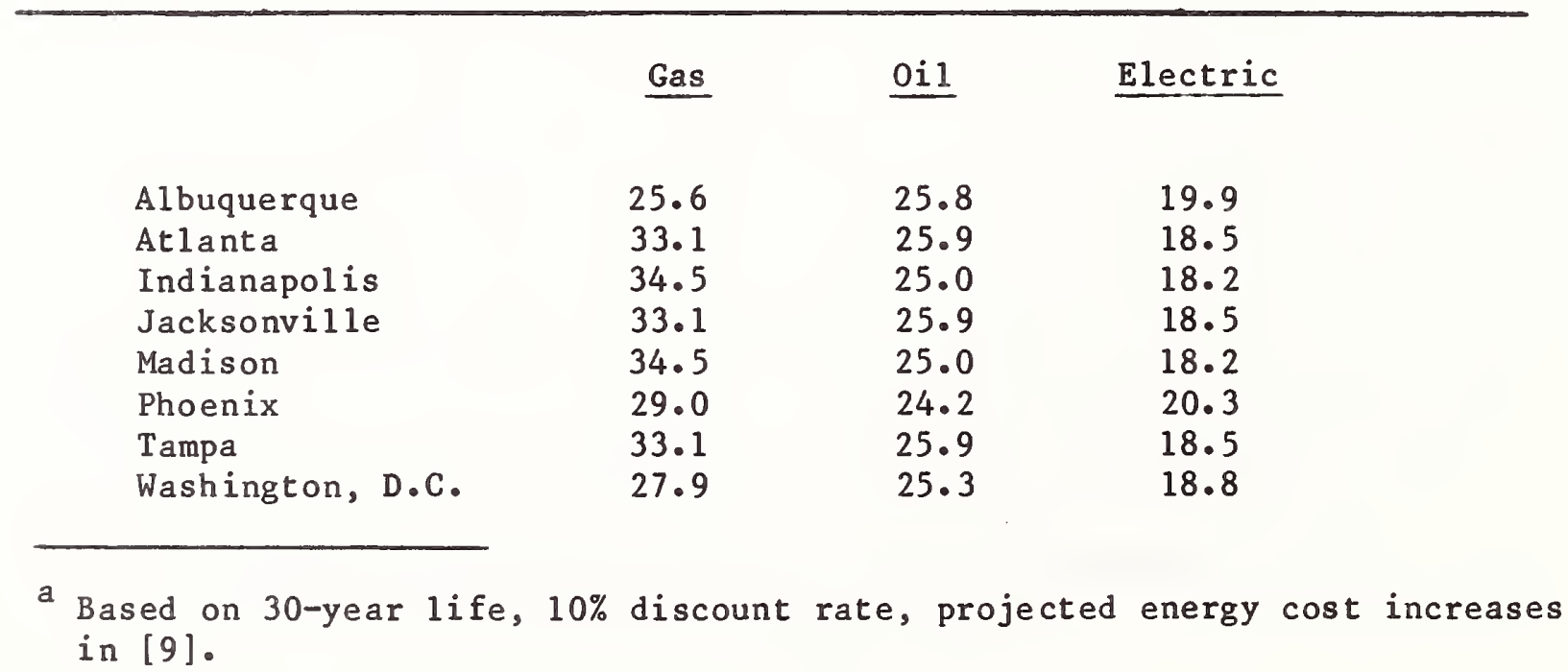


Table 10. Insulation Cost Data ${ }^{a}$

\begin{tabular}{|c|c|c|c|c|c|}
\hline \multirow[b]{2}{*}{ Code } & \multirow[b]{2}{*}{ Name } & \multicolumn{4}{|c|}{ Cost } \\
\hline & & $\$ / f t^{2}$ & $\left(\$ / m^{2}\right)$ & 1120 & $\mathrm{ft}^{2}\left(104 \mathrm{~m}^{2}\right)^{\mathrm{b}}$ \\
\hline B3, B4 & Reflective foil backing & 0.09 & $(0.97)$ & & $\$ 101$ \\
\hline $\mathrm{C} 3, \mathrm{C} 4$ & $3 / 4^{\prime \prime}(19 \mathrm{~mm})$ isocyanurate & 0.51 & $(5.49)$ & & 571 \\
\hline EXTRA & $\begin{array}{l}1 / 2^{\prime \prime}(13 \mathrm{~mm}) \text { isocyanurate, } 1^{\prime \prime} \\
(25 \mathrm{~mm}) \text { reflective air space }\end{array}$ & 0.53 & $(5.70)$ & & 597 \\
\hline D3, D4 & $3 / 4^{\prime \prime}(19 \mathrm{~mm})$ polystyrene & 0.33 & $(3.55)$ & & 370 \\
\hline F2, F5 & $1-1 / 2^{\prime \prime}(38 \mathrm{~mm})$ polystyrene & 0.48 & $(5 \cdot 17)$ & & 538 \\
\hline-- & Cores filled with perlite & 0.48 & $(5 \cdot 17)$ & & 538 \\
\hline $\mathrm{R}-11$ & $\begin{array}{l}\text { R-11 (R-metric } 1.9) \\
\text { fiberglass in wood frame }\end{array}$ & 0.19 & $(2.05)$ & & 213 \\
\hline $\mathrm{R}-13$ & $\begin{array}{l}\text { R-13 (R-metric } 2.3 \text { ) } \\
\text { fiberglass in wood frame }\end{array}$ & 0.25 & $(2.69)$ & & 280 \\
\hline $\mathrm{R}-19$ & $\begin{array}{l}\text { R-13 (R-metric } 2.3) \text { fiberglass } \\
\text { and } 1^{\prime \prime}(25 \mathrm{~mm}) \text { polystyrene } \\
\text { sheathing }\end{array}$ & 0.45 & $(4.84)$ & & 504 \\
\hline
\end{tabular}

\footnotetext{
a Includes materials, installation, overhead and profit

b Costs are applicable to gross wall area.
} 
If an increase in the insulation level of the wall can reduce peak heating or cooling loads to the point where smaller heating and cooling equipment can be used, the dollar savings in equipment procurement, if any, should be deducted from the cost of the insulation. While a substantial reduction in peak heating requirements is realized, in general the savings in heating equipment cost is likely to be insignificant. The reduction in peak cooling loads due to an additional increment of insulation is generally small (less than $1000 \mathrm{Btu} / \mathrm{h}(300 \mathrm{~W})$ ) and thus no reduction in cooling equipment tonnage is expected. (Cooling equipment is typically sized in approximately $6000 \mathrm{Btu} / \mathrm{h}(1800 \mathrm{~W})$ output capacity increments.) As a result, no increase in the wall insulation level above the maximum cost-effective level determined through the analysis of energy savings alone is generally found to be cost effective due to the additional savings from reductions in equipment sizing. (It is possible to construct a specific example where additional insulation would be cost effective because of a reduction in equipment size made possible by the reduction in design loads. However, no generalization can be made from such an example.)

A summary computer program was developed to allow the user to perform the incremental life-cycle-cost analysis of alternative insulation strategies for any wall type on which thermal performance data are available. The user enters the base (i.e., uninsulated) U-value of the appropriate wall system type, the name, corresponding wall U-value and total cost for each of the increasing insulation levels to be analyzed. Savings due to reductions in heating requirements are always calculated. However, the 
user can specify whether to consider (1) cooling savings based on cooling only when the outdoor temperature is above the thermostat setpoint, (2) no cooling savings due to insulation, or ( 3 ) net cooling savings (losses) regardless of the outdoor temperature (i.e., no natural ventilation). The program uses the thermal performance data developed with NBSLD analysis for the appropriate wall system in order to interpolate the thermal performance of the insulated wall systems to be analyzed. It then determines the incremental change in heating and cooling requirements (as specified by the user) due to each additional level of insulation. Using the method shown above it calculates the incremental life-cycle heating and cooling savings and compares their sum to the incremental insulation costs. Both the incremental net savings (incremental savings minus incremental cost) and cumulative net savings to that point are shown. The level of insulation with the greatest cumulative net savings is the most cost-effective level for the climatic and cost conditions considered. Any additional level(s) of insulation will not provide incremental savings greater than the costs incurred. If two or more alternative methods of insulating the same wall are to be evaluated, the method with the greatest cumulative net savings is the most cost effective.

\subsection{RESULTS OF THERMAL AND ECONOMIC ANALYSIS}

In order to evaluate the cost effectiveness of the insulation measures specified by the sponsor, the analysis was divided into five insulation groups, designated as:

HUD 1 - insulation of hollow 8" (200 mm) block walls with reflective air space and isocyanurate (B3, C3, "EXTRA") 
HUD 2 - insulation of hollow 8" (200 mm) block walls with reflective air space and polystyrene (B3, D3, F2)

HUD 3 - insulation of $8^{\prime \prime}(200 \mathrm{~mm})$ block walls with cores filled, reflective air space and isocyanurate (B4, C4)

HUD 4 - insulation of $8^{\prime \prime}(200 \mathrm{~mm})$ block walls with cores filled, reflective air space and polystyrene (B4, D4, F5)

HUD 5 - insulation of wood-frame walls with fiberglass ( $R-11$ and $\mathrm{R}-13$ ) and polystyrene sheathing ( $R-19)$ (R-metric 1.9, 2.3 and 3.3 ).

Each group was analyzed to determine the most cost-effective level of insulation, starting with an uninsulated wall with a non-reflective air space. All cases were analyzed with the assumption of no cooling savings. A number of cases, including Phoenix, Tampa, and Jacksonville were analyzed where the effects of insulation on cooling requirements were calculated as well in order to determine whether this had any significant effect on the results. Computer printouts for each run made are included in Appendix A (no cooling savings), Appendix B (cooling based on windows closed) and Appendix C (cooling savings above $78^{\circ} \mathrm{F}\left(25.5^{\circ} \mathrm{C}\right.$ ) outdoor temperature). All printouts in the appendices are calculated using the base insulation cost data shown in Table 10. Tabulations of these runs are presented in Table 11. The tabulations show the optimal (i.e., most cost-effective) level of insulation for each group along with its corresponding life-cycle net savings. All data in Table 11 are based on no cooling savings from insulation, except for Phoenix, where the data are based on the thermal analysis with windows closed at all times (C). In other cities, the assumption of no cooling savings generally provides the same results in terms of optimal insulation levels as the two other as sumptions regarding cooling savings. Table 12 presents similar tabulations based on the 25 percent increase in insulation costs. 
Table 11. OPT DMAL INSULATION LEVEL AND NET SAVINGS ${ }^{a}$ BY FURNACE TYPE, CITY, AND INSULATION GROUP (INSULATION PRICES BASED ON TABLE 10)

A. GAS FURNACE

\begin{tabular}{|c|c|c|c|c|c|}
\hline & & & TION $G$ & & \\
\hline & CORE & EN & CORE & ILLED & WOOD-FRAME \\
\hline & HUD 1 & HUD 2 & HUD 3 & HUD 4 & HUD 5 \\
\hline CITY & & & & & \\
\hline Al buquerque & B3 & B3 & 0 & 0 & $\mathrm{R}-11^{\mathrm{C}}$ \\
\hline & $\$ 294$ & $\$ 294$ & $\$ 0$ & $\$ 0$ & $\$ 362$ \\
\hline Atlanta & B3 & B3 & 0 & 0 & R-11 \\
\hline & 282 & 282 & 0 & 0 & 350 \\
\hline Indianapolis & EXTRA & F2 & B4 & B4 & R-19 \\
\hline & 1048 & 1151 & 926 & 926 & 1268 \\
\hline Jacksonville & B3 & B3 & 0 & 0 & 0 \\
\hline & 21 & 21 & 0 & 0 & 0 \\
\hline Madison & EXTRA & F2 & B4 & F5 & $R-19$ \\
\hline & 1441 & 1554 & 1303 & 1306 & 1701 \\
\hline Phoenix & B 3 & B3 & 0 & 0 & $R-11$ \\
\hline & 222 & 222 & 0 & 0 & 441 \\
\hline Tampa & 0 & 0 & 0 & 0 & 0 \\
\hline & 0 & 0 & 0 & 0 & 0 \\
\hline Washington & B3 & B3 & B4 & B4 & $R-11$ \\
\hline & 561 & 561 & 375 & 375 & 726 \\
\hline
\end{tabular}

B. OIL FURNACE

\begin{tabular}{|c|c|c|c|c|c|c|}
\hline & & \multicolumn{5}{|c|}{ INSULATION GROUP } \\
\hline & & \multicolumn{2}{|c|}{ CORES OPEN } & \multicolumn{2}{|c|}{ CORES FILLED } & \multirow{2}{*}{$\begin{array}{l}\text { WOOD-FRAME } \\
\text { HUD } 5 \\
\end{array}$} \\
\hline & & HUD 1 & HUD 2 & HUD 3 & HUD 4 & \\
\hline \multicolumn{7}{|l|}{ CITY } \\
\hline Albuquerque & & $\begin{array}{c}\text { B3 } \\
\$ 621\end{array}$ & $\begin{array}{c}\text { F2 } \\
\$ 640\end{array}$ & $\begin{array}{c}\text { B4 } \\
\$ 494\end{array}$ & $\begin{array}{c}\text { B4 } \\
\$ 494\end{array}$ & $\begin{array}{l}R-11 \\
\$ 838\end{array}$ \\
\hline Atlanta & f & $\begin{array}{l}\text { B3 } \\
386\end{array}$ & $\begin{array}{l}\text { B3 } \\
386\end{array}$ & $\begin{array}{l}\text { B4 } \\
138\end{array}$ & $\begin{array}{l}\text { B4 } \\
138\end{array}$ & $\begin{array}{r}R-11 \\
504\end{array}$ \\
\hline Indianapolis & & $\begin{array}{l}\text { EXTRA } \\
1125\end{array}$ & $\begin{array}{c}\text { F2 } \\
1229\end{array}$ & $\begin{array}{l}\text { B4 } \\
999\end{array}$ & $\begin{array}{l}\text { B4 } \\
999\end{array}$ & $\begin{array}{l}R-19 \\
1350\end{array}$ \\
\hline Jacksonville & & $\begin{array}{l}\text { B3 } \\
40\end{array}$ & $\begin{array}{l}\text { B3 } \\
40\end{array}$ & $\begin{array}{l}0 \\
0\end{array}$ & $\begin{array}{l}0 \\
0\end{array}$ & $\begin{array}{c}R-11 \\
16\end{array}$ \\
\hline Madison & & $\begin{array}{l}\text { EXTRA } \\
1593\end{array}$ & $\begin{array}{c}F 2 \\
1709\end{array}$ & $\begin{array}{c}\text { B4 } \\
1448\end{array}$ & $\begin{array}{c}\text { F5 } \\
1483\end{array}$ & $\begin{array}{l}R-19 \\
1865\end{array}$ \\
\hline Phoenix & & $\begin{array}{l}\text { B3 } \\
250\end{array}$ & $\begin{array}{l}\text { B3 } \\
250\end{array}$ & $\begin{array}{l}\text { B4 } \\
38\end{array}$ & $\begin{array}{l}\text { B4 } \\
38\end{array}$ & $\begin{array}{r}R-11 \\
490\end{array}$ \\
\hline Tampa & & $\begin{array}{l}0 \\
0\end{array}$ & $\begin{array}{l}0 \\
0\end{array}$ & $\begin{array}{l}0 \\
0\end{array}$ & $\begin{array}{l}0 \\
0\end{array}$ & $\begin{array}{l}0 \\
0\end{array}$ \\
\hline Washington & & $\begin{array}{l}\text { B3 } \\
654\end{array}$ & $\begin{array}{l}\text { F2 } \\
696\end{array}$ & $\begin{array}{l}\text { B4 } \\
517\end{array}$ & $\begin{array}{l}B 4 \\
517\end{array}$ & $\begin{array}{r}R-11 \\
858\end{array}$ \\
\hline
\end{tabular}

a Net savings = present dollar value of energy savings over 30 years less cost of insulation.

b Tabulated from Appendices A and B. See Table 1 for description of insulation codes.

c $R-11=\mathrm{R}$-metric $1.9, \mathrm{R}-19=\mathrm{R}$-metric 3.3 . 
TABLE 11. (Continued)

C. ELECTRIC FURNACE

\begin{tabular}{|c|c|c|c|c|c|}
\hline \multirow[b]{2}{*}{ CITY } & BUD 1 & HUD 2 & HUD 3 & HUD 4 & KUD 5 \\
\hline & & & & & \\
\hline Albuquerque & $\begin{array}{l}\text { EXTRA } \\
\$ 1514\end{array}$ & $\begin{array}{c}\text { F2 } \\
\$ 1627\end{array}$ & $\begin{array}{c}\text { B4 } \\
\$ 1444\end{array}$ & $\begin{array}{c}\text { B4 } \\
\$ 1444\end{array}$ & $\begin{array}{l}R-19 \\
\$ 1817\end{array}$ \\
\hline Atlanta & $\begin{array}{l}\text { B3 } \\
693\end{array}$ & $\begin{array}{l}\text { F2 } \\
759\end{array}$ & $\begin{array}{l}B 4 \\
627\end{array}$ & $\begin{array}{l}\text { B4 } \\
627\end{array}$ & $\begin{array}{r}\text { R-11 } \\
955\end{array}$ \\
\hline Indianapolis & $\begin{array}{l}\text { EXTRA } \\
2827\end{array}$ & $\begin{array}{c}\text { F2 } \\
2976\end{array}$ & $\begin{array}{c}C 4 \\
2645\end{array}$ & $\begin{array}{c}\text { F5 } \\
2924\end{array}$ & $\begin{array}{l}R-19 \\
3183\end{array}$ \\
\hline Jacksonville & $\begin{array}{l}\text { B3 } \\
128\end{array}$ & $\begin{array}{l}\text { B3 } \\
128\end{array}$ & $\begin{array}{l}0 \\
0\end{array}$ & $\begin{array}{l}0 \\
0\end{array}$ & $\begin{array}{r}R-11 \\
160\end{array}$ \\
\hline Madison & $\begin{array}{l}\text { EXTRA } \\
3645\end{array}$ & $\begin{array}{c}F 2 \\
3815\end{array}$ & $\begin{array}{c}\text { C4 } \\
3545\end{array}$ & $\begin{array}{c}\text { F5 } \\
3881\end{array}$ & $\begin{array}{l}R-19 \\
4084\end{array}$ \\
\hline Phoenix & $\begin{array}{l}\text { B3 } \\
435\end{array}$ & $\begin{array}{l}\text { B3 } \\
435\end{array}$ & $\begin{array}{l}B 4 \\
363\end{array}$ & $\begin{array}{l}\text { B4 } \\
363\end{array}$ & $\begin{array}{r}\text { R-11 } \\
819\end{array}$ \\
\hline Tampa & $\begin{array}{l}0 \\
0\end{array}$ & $\begin{array}{l}0 \\
0\end{array}$ & $\begin{array}{l}0 \\
0\end{array}$ & $\begin{array}{l}0 \\
0\end{array}$ & $\begin{array}{l}0 \\
0\end{array}$ \\
\hline Washington & $\begin{array}{l}\text { EXTRA } \\
2035\end{array}$ & $\begin{array}{c}\text { F2 }_{2} \\
2161\end{array}$ & $\begin{array}{c}\text { B4 } \\
1889\end{array}$ & $\begin{array}{c}\text { F5 } \\
1998\end{array}$ & $\begin{array}{l}R-19 \\
2300\end{array}$ \\
\hline
\end{tabular}

D. HEAT PUMP

INSULATION GROUP

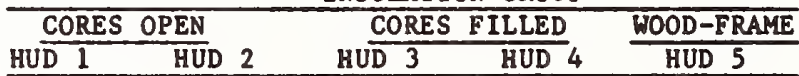

\section{$\underline{\operatorname{CITY}}$}

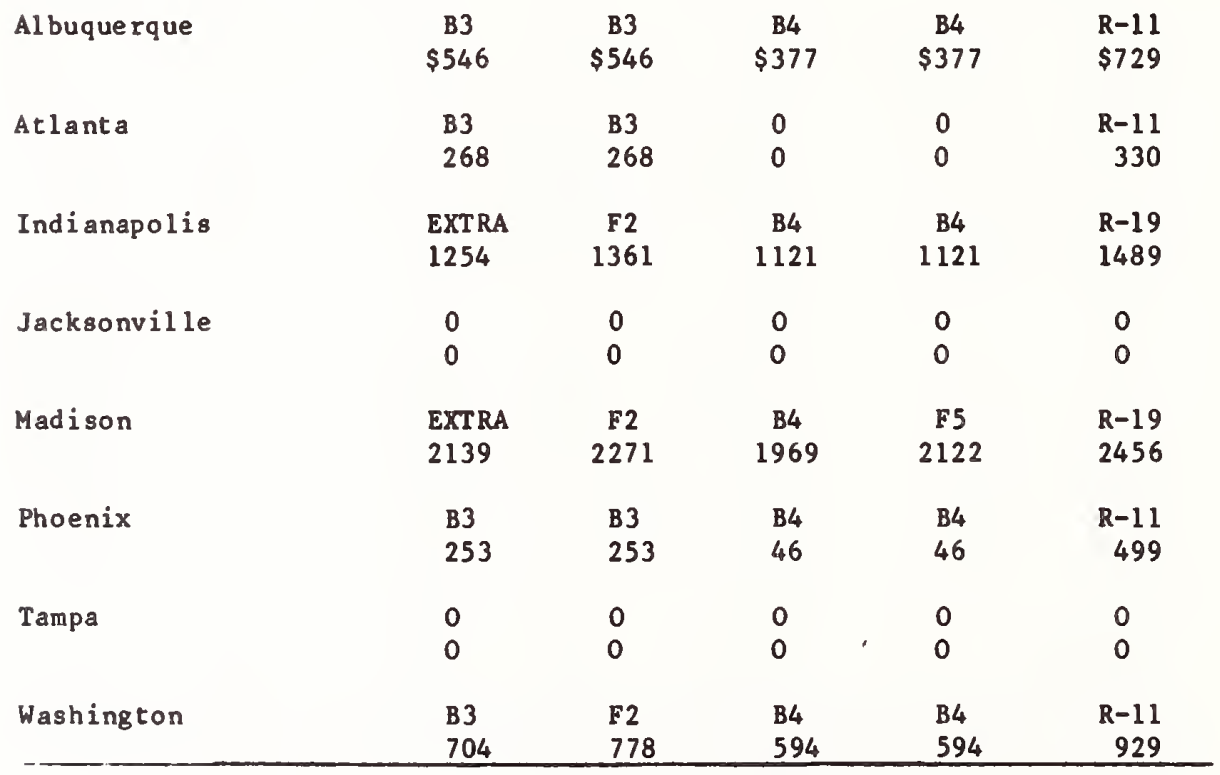

42 
Table 12. OPT DMAL INSULATION LEVEL AND NET SAVINGS BY FURNACE TYPE, CITY, AND INSULATION GROUP (INSULATION PRICES BASED ON TABLE $10+25 \%$ )

\section{A. GAS FURNACE}

\begin{tabular}{|c|c|c|c|c|}
\hline \multicolumn{5}{|c|}{ INSULATION GROUP } \\
\hline CORES & OPEN & CORES & FILLED & WOOD-FRAME \\
\hline HOD I & HUD 2 & HUD 3 & HUD 4 & मUं 5 \\
\hline
\end{tabular}

\section{CITY}

Albuquerque

$\begin{array}{cccc}\text { B3 } & \text { B3 } & 0 & \\ \$ 268 & \$ 268 & \$ 0 & \$ 0\end{array}$

Atlanta

B3

$256 \quad 256$

0

0

0
0 HUD 5

Ind i a napolis

$\begin{array}{cc}\text { EXTRA } & \text { F } \\ 899 & 1016\end{array}$

B4 B4

B4
766

$$
\text { R-11 }
$$
$\$ 309$

Jacksonville

$\begin{array}{ll}0 & 0 \\ 0 & 0\end{array}$

0
0

0
0

0
0

R-11

Madison

EXTRA F2

\section{B4} 1144

B4 1144

296

Phoenix

B3 B3

$197 \quad 197$

0

0

R-11

1205

Tampa

$\begin{array}{ll}0 & 0 \\ 0 & 0\end{array}$

$\begin{array}{ll}0 & 0 \\ 0 & \end{array}$

0
0

0
0

0

Washington

B3 B3

B3

B4

B4

215

B. OIL FURNACE

INSULATION GROUP

\begin{tabular}{lllll}
\hline CORES OPEN & CORES FILLED & WOOD -FRAME \\
HUD 1 & HUD & HUD 3 & HUD & HUD 5 \\
\hline
\end{tabular}

\section{CITY}

Albuquerque

$$
\text { B3 }
$$

$\$ 596$

B3
$\$ 596$

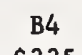

B4

R-11

$$
\text { B3 }
$$

Atlanta

B3
361

B3

$\$ 335$

$\$ 335$

$\$ 785$

Indi anapolis

EXTRA
976

Jacksonville

$$
\begin{aligned}
& \text { B3 } \\
& 14
\end{aligned}
$$

Madison

Phoenix

$\begin{array}{lc}\text { EXTRA } & \text { F2 } \\ 1443 & 1575\end{array}$

(

$\begin{array}{rr}0 & \mathrm{R}-11 \\ 0 & 450\end{array}$

Phoenix

$\begin{array}{ll}\text { B3 } & \text { B3 } \\ 225 & 225\end{array}$

Tampa

Washington

0

0

$\begin{array}{ll}\text { B3 } & \text { B3 } \\ 629 & 629\end{array}$

$\begin{array}{ll}\text { B4 } & \text { B4 } \\ 357 & 357\end{array}$

0
0

0

B4 B4

1288

R-19

$\begin{array}{lll}1288 & 1288 & 1739\end{array}$
Based on Appendices $A$ and B. See Table 1 for description of insulation
codes. 
TABLE 12. (Continued)

C. ELECTRIC FURNACE

\begin{tabular}{|c|c|c|c|c|}
\hline \multicolumn{5}{|c|}{ INSULATION GROUP } \\
\hline CORE & $\overline{\mathrm{PEN}}$ & CORE & ILLED & WOOD-FRAME \\
\hline HUD 1 & HUD $\cdot 2$ & HUD 3 & HUD 4 & HUD 5 \\
\hline
\end{tabular}

$\underline{\text { CITY }}$

Albuque rque

$\begin{array}{ccccr}\text { EXTRA } & \text { F2 } & \text { B4 } & \text { B4 } & \text { R-19 } \\ \$ 1365 & \$ 1493 & \$ 1284 & \$ 1284 & \$ 1691\end{array}$

Atlanta

$\begin{array}{lllll}\text { B3 } & \text { B3 } & \text { B4 } & \text { B4 } & R-19\end{array}$

$\begin{array}{lllll}668 & 668 & 467 & 467 & 902\end{array}$

Indianapolis

$\begin{array}{lllll}\text { EXTRA } & \text { F2 } & \text { B4 } & \text { F5 } & \text { R-19 }\end{array}$

$\begin{array}{lllll}2678 & 2842 & 2458 & 2655 & 3057\end{array}$

Jacksonville

B3 B

$103 \quad 103$

$\begin{array}{lll}0 & 0 & R-11\end{array}$

Madison

$\begin{array}{llccc}\text { EXTRA } & \text { F2 } & \text { C4 } & \text { F5 } & \text { R-19 }\end{array}$

$\begin{array}{lllll}3495 & 3681 & 3268 & 3612 & 3958\end{array}$

Phoenix

$\begin{array}{lllll}\text { B3 } & \text { B3 } & \text { B4 } & \text { B4 } & R-11\end{array}$

$\begin{array}{llllr}\text { B3 } & \text { B3 } & \text { B4 } & \text { B4 } & R-11 \\ 409 & 409 & 203 & 203 & 765\end{array}$

Tampa

0

(

(0

765

Washington

$\begin{array}{lll}0 & 0 \\ 0 & 0\end{array}$

$\begin{array}{lllll}\text { EXTRA } & \text { F2 } & \text { B4 } & \text { B4 } & \text { R-19 }\end{array}$

$\begin{array}{lllll}1886 & 2026 & 1730 & 1730 & 2174\end{array}$

D. HEAT PUMP

INSULATION GROUP

\begin{tabular}{llll}
\hline CORES OPEN & CORES FILLED & WOOD-FRAME \\
\hline HUD 1 & HUD 2 & HUD 3 HUD 4 & HUD 5 \\
\hline
\end{tabular}

CITY

Albuquerque

$$
\text { B3 }
$$

$\$ 521$

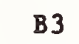

$\$ 521$

B4

$\$ 217$

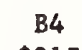

$\$ 217$

R-19

Atlanta

$\begin{array}{ll}\text { B3 } & \text { B3 } \\ 243 & 243\end{array}$

0

0
0

0

$\$ 676$

Indianapolis

EXTRA F2

$1104 \quad 1227$

B4 B4

R-11

1104

Jacksonville

0

0
0

962

Madison

EXTRA F2

$1990 \quad 2136$

0
0

962

R-11

B 3 B3

Phoenix

$$
228
$$

B3

Tampa

Washington

0
0

0

B3 B3

$679 \quad 679$

B4 F5 R-19

$\begin{array}{lll}B 4 & F & 1853\end{array}$

$0 \quad 0 \quad \mathrm{R}-11$


In the cases where insulation is added to hollow block walls, the foil-backed gypsum wallboard over the $3 / 4^{\prime \prime}(19 \mathrm{~mm})$ airspace (B3)* appears to be the most cost-effective insulation level for gas, oil, and heat pump in the milder climates while either the $1 / 2^{\prime \prime}(13 \mathrm{~mm})$ isocyanurate with foil-backed gypsum board and air space ("EXTRA") or the 1-1/2" (38 mm) polystyrene insulation (F2) appears cost effective for most applications in the colder climates and where an electric furnace is used. The use of either $3 / 4^{\prime \prime}(19 \mathrm{~mm})$ isocyanurate (C3) or $3 / 4^{\prime \prime}(19 \mathrm{~mm})$ polystyrene (D3) is never cost effective when compared to the $3 / 4^{\prime \prime}(19 \mathrm{~mm})$ reflective airspace (B3) because the additional savings are relatively minor and the additional cost relatively high. In the cases where the cores of the $8 "(200 \mathrm{~mm})$ blocks are filled with perlite insulation, the foil-backed gypsum wallboard appears most cost effective in all but the coldest climates with an electric furnace. However, the cumulative net savings for the cases where core insulation is examined (HUD 3, HUD 4) are less than those for the cases with no insulation in the cores (HUD 1 and HUD 2). This shows that it is generally more cost effective to insulate the inside wall and leave the cores empty. In general, the $8^{\prime \prime}(200 \mathrm{~mm})$ block wall with $1-1 / 2^{\prime \prime}$ (38 mm) of polystyrene insulation and hollow cores (F2) has a lower U-value and higher net savings than the same wall with $1 / 2^{\prime \prime}(13 \mathrm{~mm})$ isocyanurate insulation and a reflective air space (EXTRA).

* Potentially, condensation on the foil-backed gypsum wallboard during the air conditioning season in locations such as the Gulf Coast, or dust deposits in the drier regions of the U.S. could degrade the longterm thermal performance of this insulation technique. However, these phenomena have not been widely noted as problems in the field. 
In the colder climates, even more insulation than has been examined may be cost effective on the inside. However, beyond $1-1 / 2^{\prime \prime}$ (38 mm) insulation the additional cost of the insulation should be adjusted to offset the effects of the reduced living area. This adjustment can be made by determining the additional cost of moving the end wall outwards by several inches in order to maintain an equivalent interior area.

A minimum of $R-11$ (R-metric 1.9) insulation in wood-frame walls appears cost effective in all locations except Tampa and in Jacksonville with gas heat or a heat pump. $R-19$ (R-metrioc 3.3) insulation appears cost effective in all electrically heated houses except in southern areas and in gas-, oil-, and heat pump-heated houses in northern areas of the U.S.

Similar patterns appear in the analysis using installed insulation costs increased by 25 percent. Foil-backed gypsum is indicated in slightly colder climates than indicated using the lower costs. Results for insulation in wood-frame walls using the higher insulation costs are nearly identical to those based on the lower costs.

Sensitivity analysis to the house orientation was performed in order to determined the effects of rotation on the cost effectiveness of the insulation in masonry walls. The house was rotated $90^{\circ}$ to the west, so that the long walls ran north-to-south and the largest window exposure was to the west instead of south. NBSLD simulation of the $8^{\prime \prime}(200 \mathrm{~mm})$ hollow block wall were made for Phoenix, Washington, and Madison. Annual heating and cooling requirements were increased by as much as 17 percent and 12 percent respectively over the same house in its original orientation. The savings due to 
insulating the exterior walls of the rotated house were slightly, but not significantly, greater than those resulting from insulation of the walls in the original orientation. As a result, the maximum costeffective level of insulation did not change with respect to building orientation. The economic analysis for these three cities are provided in Appendix $D$.

\subsection{CONCLUSIONS}

Although the energy savings due to an equivalent decrease in U-value for masonry walls are nearly the same as those for wood-frame walls, the maximum economic level of insulation is significantly lower for the masonry walls. This is due to the fact that the insulation of masonry walls is significantly more expensive than insulation in wood-frame walls, thereby reducing the cost effectiveness of the insulation. As a result, houses with optimally insulated $8 "(200 \mathrm{~mm})$ concrete block walls (100 pcf, $1600 \mathrm{~kg} / \mathrm{m}^{3}$ ), are likely to use somewhat more energy for heating each year than optimally insulated wood-frame houses and slightly less (1 to 5 percent) for cooling.

In extreme southern climates, where heating requirements are small, foil-backed gypsum board with $3 / 4^{\circ}(19 \mathrm{~mm})$ air space is generally the maximum cost-effective insulation level in the $8 "$ (200 mm) block wall. Except in climates like that of Phoenix, which tends to have extremely hot days, insulation has only marginal effects on cooling requirements and may actually increase cooling requirements if natural ventilation is not used when adequate for cooling purposes. 
In general, insulation in the cores of the $8^{\prime \prime}(200 \mathrm{~mm})$ blocks is not as cost effective as insulation on the inside wall surface. This is because of the high cost of this method and the low change in overall thermal transmittance relative to that for insulation on the inside surfaces. Although the analysis was limited to a maximum of $1-1 / 2$ " (38 $\mathrm{mm}$ ) of rigid foam insulation in the wall cavity, more may be cost effective in the colder climates, especially if electric heat is used.

It should be recognized that the results discussed in this report are limited in scope in that only an $8^{\circ}$ (200 mm) block wall with a concrete density of $100 \operatorname{pcf}\left(1600 \mathrm{~kg} / \mathrm{m}^{3}\right)$ and a conventional wood-frame wall were examined in conjunction with a limited analysis of insulation strategies. The key to improving the cost effectiveness of insulation in masonry walls appears to lie in finding lower cost methods for insulating these walls, since the savings from the insulation are quite similar to those for wood frame construction. A more complete analysis of masonry wall types and insulation strategies will be included in the final report to be prepared for DoE later in 1979. 


\section{REFERENCES}

1. Hastings, S. R., Three Proposed Typical House Designs for Energy, Conservation Research, NBSIR 77-1309, National Bureau of Standards, October 1977.

2. Kusuda, T., NBSLD, The Computer Program for Heating and Cooling Loads in Buildings, BSS-69, National Bureau of Standards, July 1976.

3. Petersen, S. R., and Barnett, J. P., NBSLD-XO, Expanded NBSLD Output for Thermal Performance Analys is of Building Components, BSS-IXX, National Bureau of Standards, (In Final Review).

4. Peavy, Powe11, Burch, Dynamic Thermal Performance of an Experimental Masonry Building, BSS -45 , National Bureau of Standards, Washington, D.C. 1972.

5. Peavy, Burch, Powe11, Hunt, Comparison of Measured and ComputerPredicted Thermal Performance of a Four Bedroom Wood-Frame Townhouse. BSS-57, National Bureau of Standards, Washington, D.C., 1973.

6. Roberts, Na11, Rogers, Greenberg, "Comparison of Computer-Predicted Thermal Loads with Measured Data from Three Occupied Townhouses," ASHRAE Transactions, Vol. 83, Part I, New York, 1978.

7. Chi, J., Kelley, G., Didion, D., "Use of a Computer Model to Evaluate Energy Savings Potentials in Gas- Fired Furnaces," Paper No. 78-IHTC-77, 1978 International Heat Transfer Conference, Toronto, an Unpublished Analyses of gas and oil fired furances with this computer model at NBS.

8. Heat Pump Technology, A Survey of Technical Development Market Prospects, HCP/M2121-01, Gordian Associates, New York, June 1978.

9. Borg, S., and Moden, R., "Historic and Forcasted Energy Prices by U.S. Department of Energy Region and Fuel Type for Three Macro economic Scenarios and one Imported Oil Price Escalation Scenario," DoE/EIA0102/27, U.S. Department of Energy, December 1978.

10. Building Construction Cost Data, 1978, R. S. Means Company, Inc., Duxbury, Massachusetts, 1977.

11. "1978 Masonry Cost Guide," Masonry Advisory Council, Park Ridge, Illinois, 1978. 

APPENDIX A

Computer Printouts of Economic Analyses

for A11 Cities Assuming

No Cooling Savings

$A-1$ 
HUD 1

ALBUQUERQUE NEW MEXICO 1200 SQ FT HOUSE IN BLOCK (OPEN COKE)

COOLING LOADS BASED ON NO COOLING SAVINGS

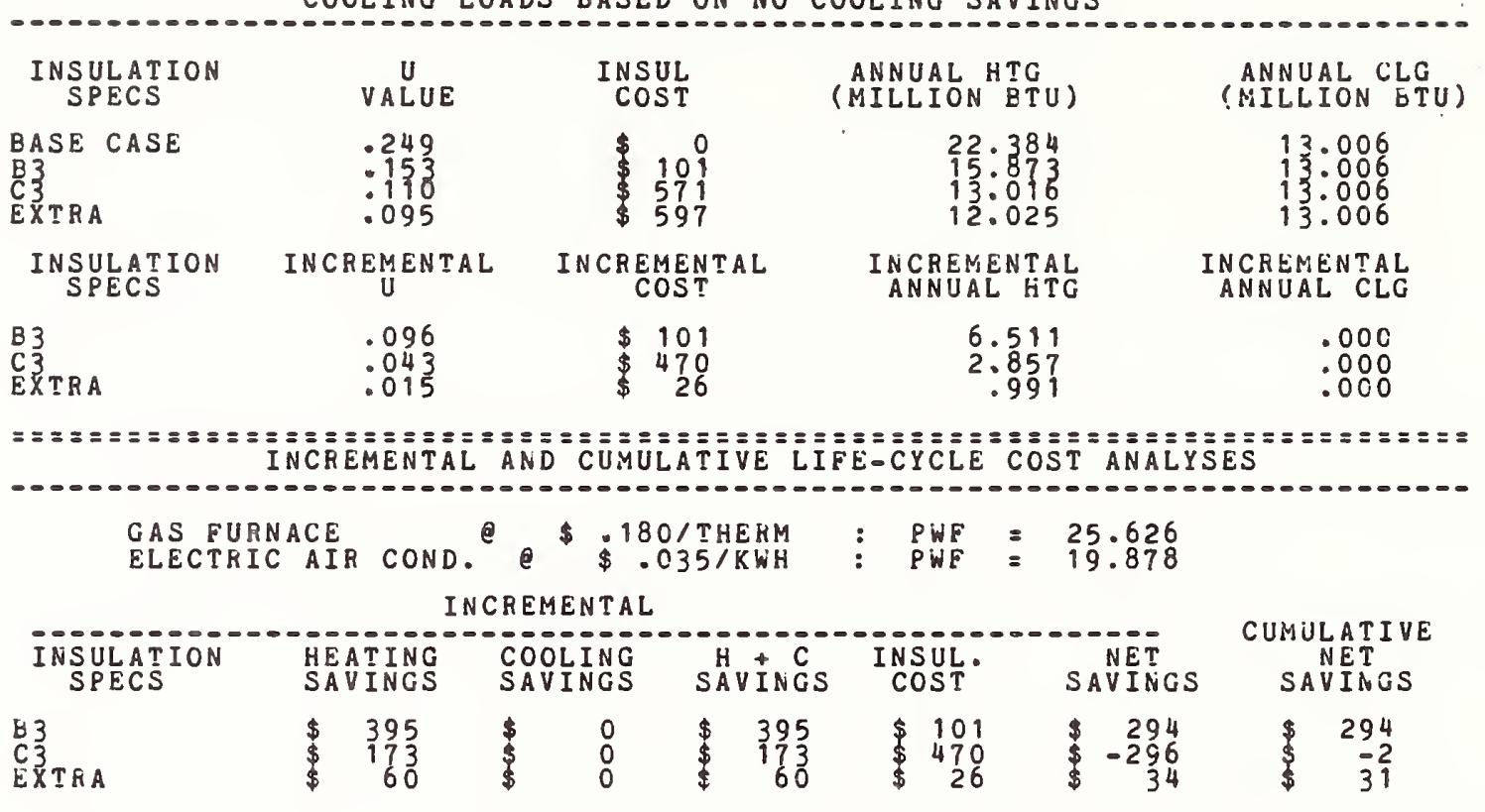

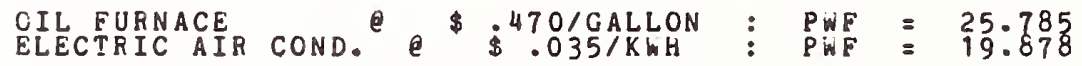

INCREMENTAL

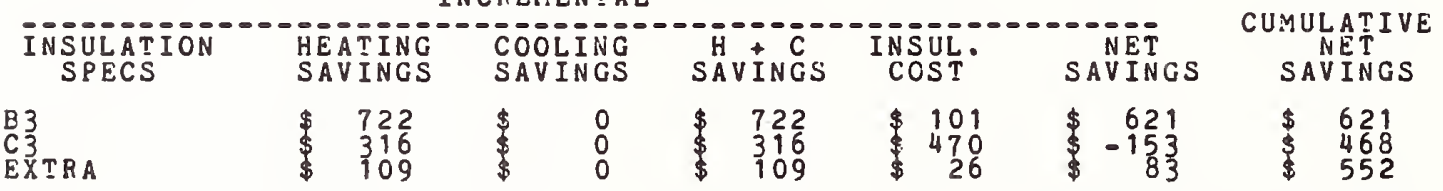

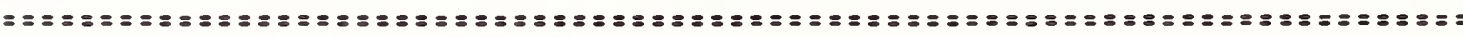

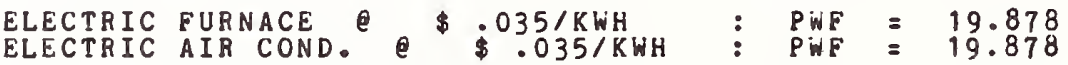

INCREMENTAL

\begin{tabular}{|c|c|c|c|c|c|c|}
\hline $\begin{array}{l}\text { INSULAT ION } \\
\text { SPECS }\end{array}$ & $\begin{array}{l}\text { HEATING } \\
\text { SAVINGS }\end{array}$ & $\begin{array}{l}\text { COOLING } \\
\text { SAVINGS }\end{array}$ & $\stackrel{H}{\text { SAVINAGS }}$ & $\begin{array}{l}\text { INSUL. } \\
\text { COST. }\end{array}$ & $\begin{array}{l}\text { NET } \\
\text { SAVINGS }\end{array}$ & SAVITGS \\
\hline $\begin{array}{l}3 \\
3 \\
X \\
X R A\end{array}$ & $\begin{array}{r}1327 \\
\$ \quad 582 \\
\$ \quad 202\end{array}$ & $\$$ & $\begin{array}{r}1327 \\
\$ \quad 582 \\
\$ \quad 202\end{array}$ & $\begin{array}{r}101 \\
\$ 470 \\
\$ 26\end{array}$ & $\begin{array}{r}1226 \\
\$ 112 \\
\$ \quad 176\end{array}$ & $\begin{array}{l}1226 \\
\$ 1338 \\
\$ 1514\end{array}$ \\
\hline
\end{tabular}

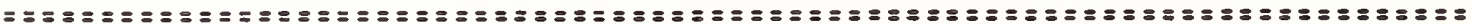

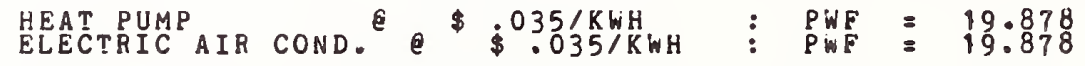

INCKEHENTAL

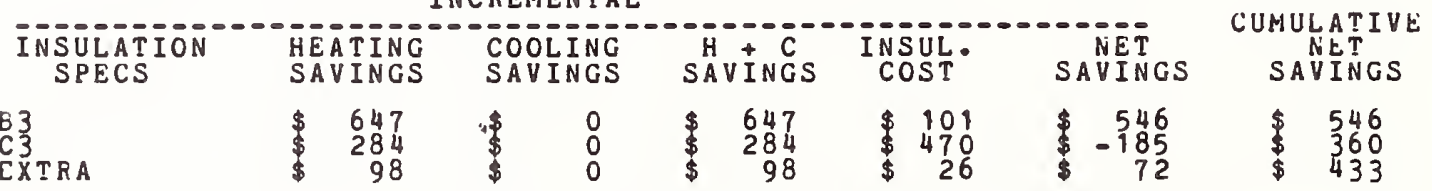

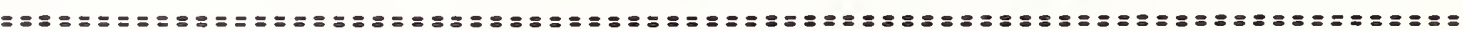


KiUD 1

ATLANTA GEORGIA 8 IN BLOCK (OPEN COKE) COOLING LOADS BASED ON NOHOUSE COOLING SAVINGS

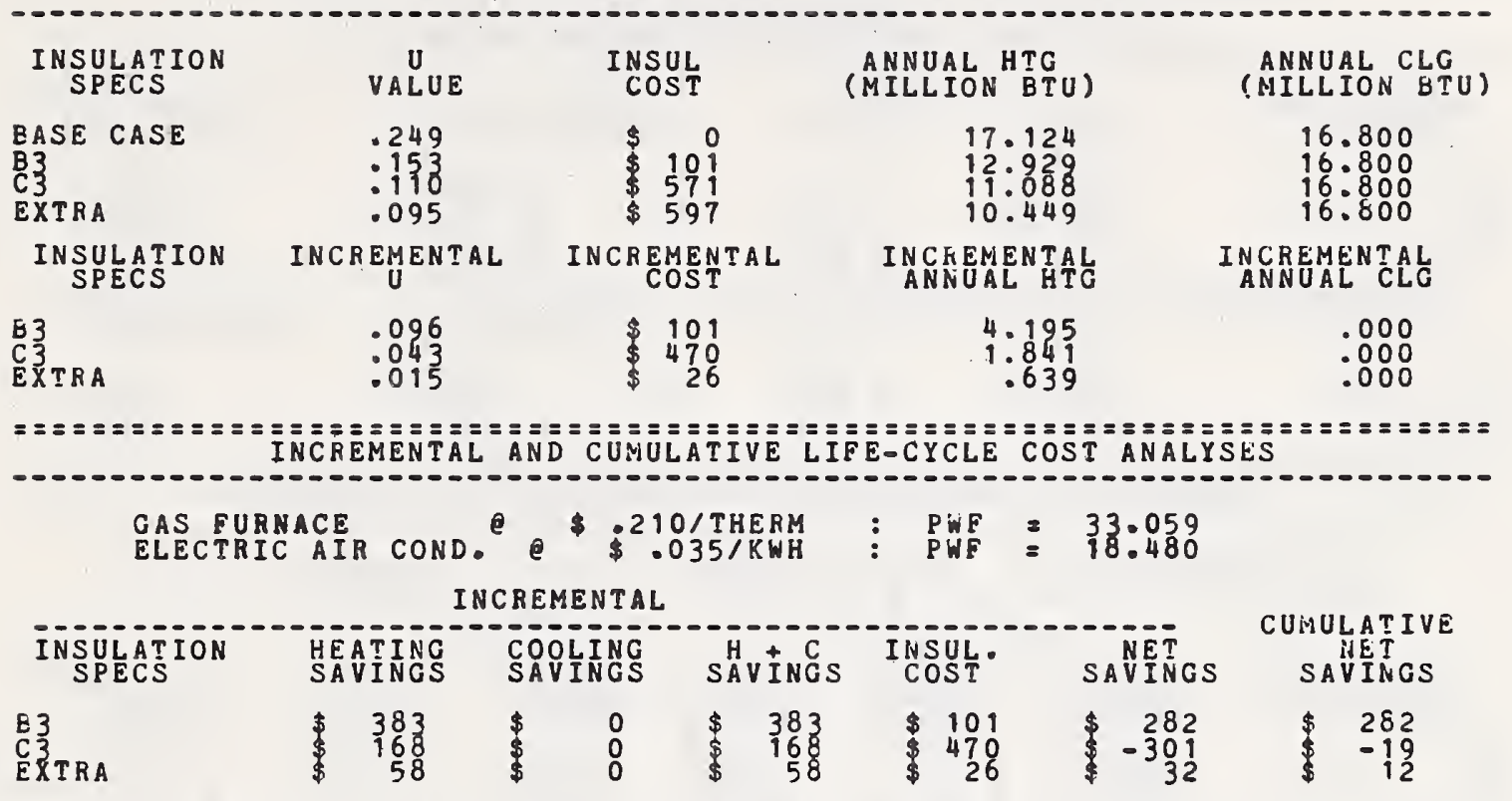

OIL FURNACE $\quad$ P $\$ 490 / G A L L O N: P W F=25.931$

ELECTRIC AIR COND. e $\$ .035 / \mathrm{KWH}: \mathrm{PWF}=18.480$

INCREMENTAL

\begin{tabular}{|c|c|c|c|c|c|c|c|}
\hline $\begin{array}{l}\text { INSULATION } \\
\text { SPECS }\end{array}$ & $\begin{array}{l}\text { HEATING } \\
\text { SAVINUS }\end{array}$ & $\begin{array}{l}\text { COOLING } \\
\text { SAVINGS }\end{array}$ & SÄVINGS & $\begin{array}{l}\text { INSUL. } \\
\text { COST }\end{array}$ & $\begin{array}{l}\text { NET } \\
\text { SAVINGS }\end{array}$ & SA & $\begin{array}{l}\text { NET } \\
\text { VINGS }\end{array}$ \\
\hline $\begin{array}{l}3 \\
3 \\
X \text { TRA }\end{array}$ & $\begin{array}{r}\quad 487 \\
\$ \quad 214 \\
\$ \quad 74\end{array}$ & $\begin{array}{l}\$ \\
\$ \\
\$\end{array}$ & $\begin{array}{r}487 \\
214 \\
74\end{array}$ & $\begin{array}{rr} & 101 \\
\$ & 470 \\
\$ & 26\end{array}$ & $\begin{array}{rr} & 386 \\
\$ & -255 \\
\$ & 48\end{array}$ & $\begin{array}{l}\$ \\
\$\end{array}$ & $\begin{array}{l}386 \\
131 \\
179\end{array}$ \\
\hline
\end{tabular}

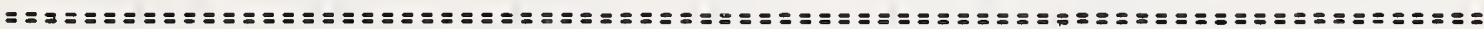

ELECTRIC FUFNACE C $\$ .035 / \mathrm{KWH}$
ELECTRIC AIR COND. $\$$ PWF $=18.480$

INCFEMENTAL

\begin{tabular}{|c|c|c|c|c|c|c|c|}
\hline INSULATION & $\begin{array}{l}\text { HEATING } \\
\text { SAVINGS }\end{array}$ & & $\begin{array}{l}\text { ING } \\
\text { NGS }\end{array}$ & SÂVINGS & $\begin{array}{l}\text { INSUL. } \\
\text { COST }\end{array}$ & SAVINGS & $\begin{array}{l}\text { CUMULATIVE } \\
\text { NETT } \\
\text { SAVINGS }\end{array}$ \\
\hline XTA & $\begin{array}{l}794 \\
\$ 348 \\
\$ \quad 121\end{array}$ & $\$$ & $\begin{array}{l}0 \\
0 \\
0\end{array}$ & $\begin{array}{l}794 \\
348 \\
121\end{array}$ & $\begin{array}{r}101 \\
\$ 470 \\
\$ \quad 26\end{array}$ & $\begin{array}{r}693 \\
\$-121 \\
\$\end{array}$ & $\begin{array}{l}\quad 693 \\
\$ 572 \\
\$ \quad 667\end{array}$ \\
\hline
\end{tabular}

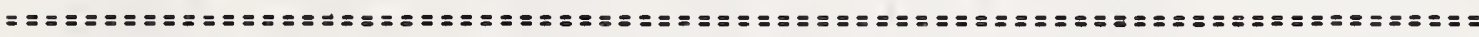
HEAT PUMP $\quad$ C $\$$.035/KWH $\quad$ PWF $=18.480$

INCHEMENTAL

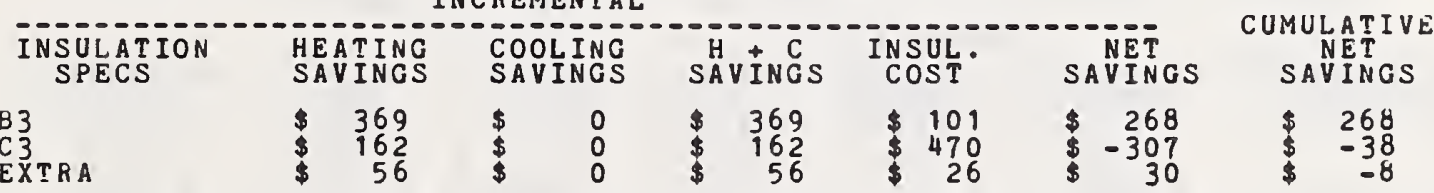

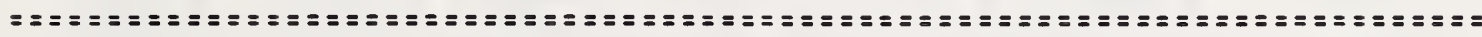


HUD 1

INDIANAPOLIS INDIANA 1200 SO FT HOUSE IN BLOCK (OPEN CORE) COOLING LOADS BASED ON NO COOLING SAVINGS

\begin{tabular}{|c|c|c|c|c|}
\hline $\begin{array}{l}\text { INSULATION } \\
\text { SPECS }\end{array}$ & $\stackrel{U}{\text { VALUE }}$ & $\begin{array}{l}\text { INSUL } \\
\text { COST }\end{array}$ & $\begin{array}{l}\text { ANNUAL HTG } \\
\text { (MILLION BTU) }\end{array}$ & $\begin{array}{l}\text { ANNUAL CLG } \\
\text { (MILLION BTU) }\end{array}$ \\
\hline $\begin{array}{l}\text { EASE CASE } \\
\text { B } 3 \\
\text { C3 } \\
\text { EXTRA }\end{array}$ & $\begin{array}{r}.249 \\
.153 \\
.110 \\
.095\end{array}$ & $\begin{array}{rr}\$ 101 \\
\$ 571 \\
\$ 597\end{array}$ & $\begin{array}{l}42.098 \\
32.157 \\
27.638 \\
26.056\end{array}$ & $\begin{array}{l}10.652 \\
10.652 \\
10.652 \\
10.652\end{array}$ \\
\hline $\begin{array}{l}\text { INSULATION } \\
\text { SPECS }\end{array}$ & INCREME & $\begin{array}{c}\text { INCREMENTAL } \\
\text { COST }\end{array}$ & $\begin{array}{l}\text { INCREMENTAL } \\
\text { ANNUAL HTG }\end{array}$ & $\begin{array}{l}\text { INCREMENTAL } \\
\text { ANNUAL CLG }\end{array}$ \\
\hline $\begin{array}{l}\text { B3 } \\
\text { C3 } \\
\text { EXTKA }\end{array}$ & $\begin{array}{l}.096 \\
.043 \\
.015\end{array}$ & $\begin{array}{rr} & 109 \\
\$ & 470 \\
\$ \quad 26\end{array}$ & $\begin{array}{l}9.941 \\
4: 518 \\
1.582\end{array}$ & $\begin{array}{l}.000 \\
.000 \\
.000\end{array}$ \\
\hline
\end{tabular}

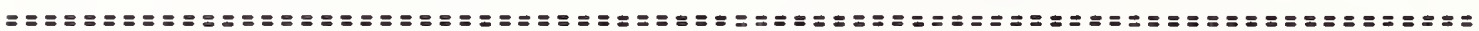
INCREMENTAL AND CUMULATIVE LIFE-CYCLE COST ANALYSES

GAS FURNACE
ELECTRIC AIR COND.

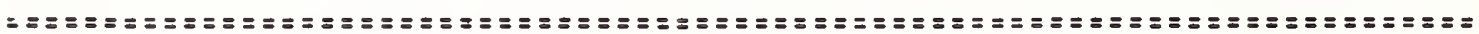

ELECTRIC FURNACE
ELECTRIC AIR COND. $\$ .040 / K W H$

INCREMENTAL

\begin{tabular}{|c|c|c|c|c|c|c|}
\hline $\begin{array}{l}\text { INSULATION } \\
\text { SPECS }\end{array}$ & $\begin{array}{l}\text { HEATING } \\
\text { SAVINGS }\end{array}$ & $\begin{array}{l}\text { COOLING } \\
\text { SAVINGS }\end{array}$ & SAVINGS & $\begin{array}{l}\text { INSUL. } \\
\text { COST }\end{array}$ & SAVINGS & $\begin{array}{c}\text { CUNULATIVE } \\
\text { NATETS } \\
\text { SAVINGS }\end{array}$ \\
\hline $\begin{array}{l}3 \\
3 \\
\text { XTRA }\end{array}$ & $\begin{array}{r}2122 \\
\$ 964 \\
\$ \quad 337\end{array}$ & $\begin{array}{l}\$ \\
\$\end{array}$ & $\begin{array}{r}2122 \\
\$ \quad 964 \\
\$ \quad 337\end{array}$ & $\begin{array}{r}\$ 101 \\
\$ 470 \\
\$ 26\end{array}$ & $\begin{array}{r}2021 \\
494 \\
\$ 311\end{array}$ & $\begin{array}{l}\quad 2021 \\
\$ 2515 \\
\$ 2827\end{array}$ \\
\hline
\end{tabular}

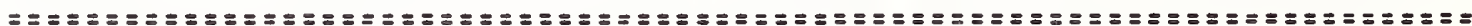

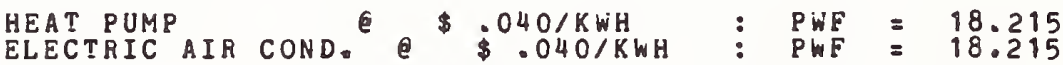

INCHEMENTAL

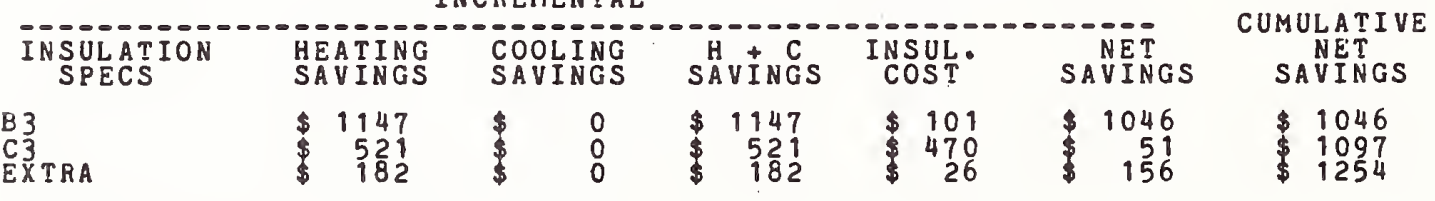

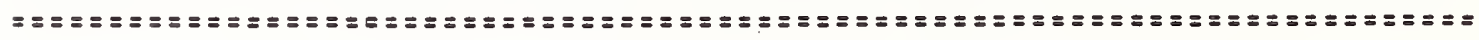


HUD 1

JACKSONVILLE FLORIDA

8 IN BLOCK (OPEN CORE) COOLING LOADS BASED ON NO COOLINC SAVINGS

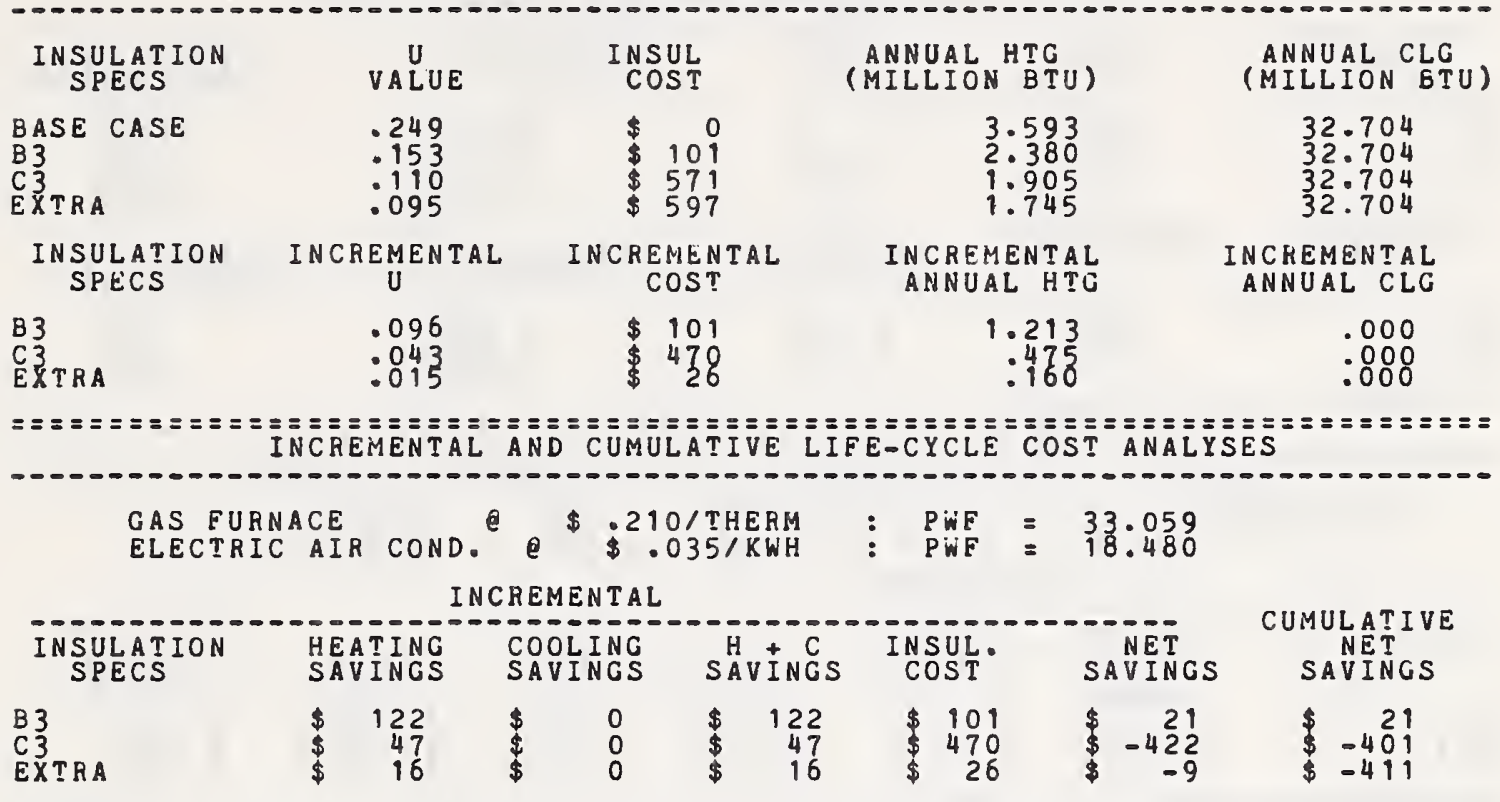

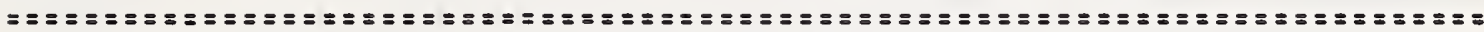

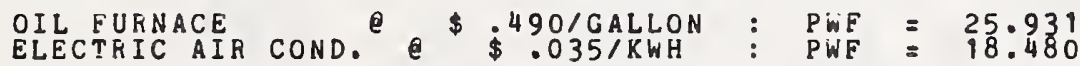

INCREMENTAL

\begin{tabular}{|c|c|c|c|c|c|c|}
\hline $\begin{array}{l}\text { INSULATION } \\
\text { SPECS }\end{array}$ & $\begin{array}{l}\text { HEATTNG } \\
\text { SAVINCS }\end{array}$ & $\begin{array}{l}\text { COOLING } \\
\text { SAVINGS }\end{array}$ & SÄVIN & $\begin{array}{l}\text { INSUL. } \\
\text { COST }\end{array}$ & SAVET INCS & SAVINGS \\
\hline $\begin{array}{l}33 \\
03 \text { TRA }\end{array}$ & $\begin{array}{r}\$ 41 \\
\$ \quad 55 \\
\$ \quad 18\end{array}$ & $\$$ & $\begin{array}{r}141 \\
55 \\
18\end{array}$ & $\begin{array}{r}101 \\
470 \\
\$ 26\end{array}$ & $\begin{array}{r}\$ 0 \\
-414 \\
-7\end{array}$ & $\begin{aligned} & 40 \\
\$ & =374 \\
- & -382\end{aligned}$ \\
\hline
\end{tabular}

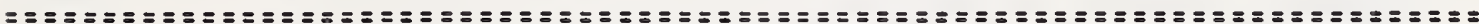

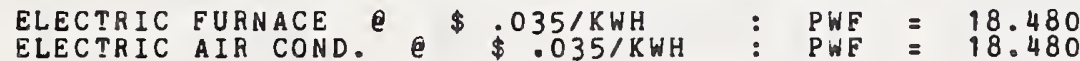

INCREMENTAL

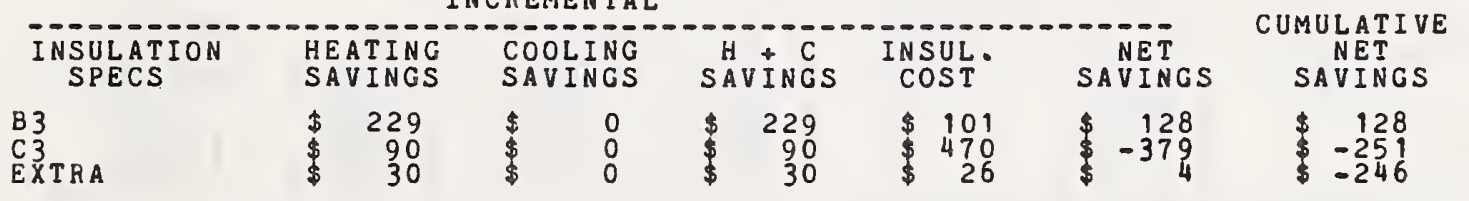

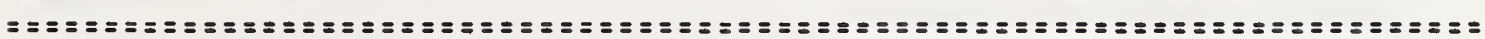

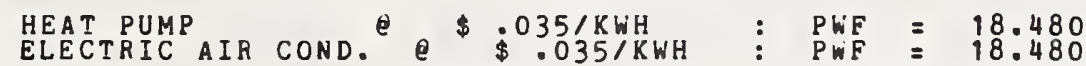
INCREMENTAL

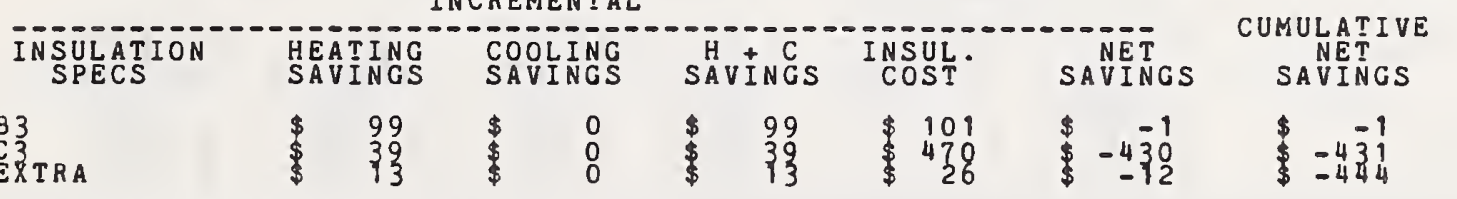

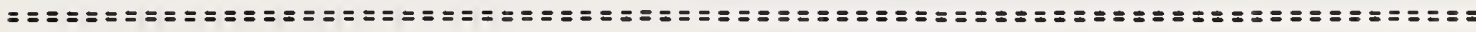


HUD 1

MADISON WISCONSIN COOLING LOADS BASED ON NOHOUSE COOLING SAVINGS

\begin{tabular}{|c|c|c|c|c|}
\hline $\begin{array}{l}\text { INSULATION } \\
\text { SPECS }\end{array}$ & $\stackrel{U}{\text { VALUE }}$ & $\begin{array}{l}\text { INSUL } \\
\text { COST }\end{array}$ & $\begin{array}{l}\text { ANNUAL HTG } \\
\text { (MILLION BTU) }\end{array}$ & $\begin{array}{l}\text { ANNUAL CLG } \\
\text { (MILLION BTU) }\end{array}$ \\
\hline $\begin{array}{l}\text { BASE CASE } \\
\text { B3 } \\
\text { C } 3 \\
\text { EXTRA }\end{array}$ & $\begin{array}{l}.249 \\
.153 \\
.110 \\
.095\end{array}$ & $\begin{array}{rr}\$ & 0 \\
\$ & 101 \\
\$ & 571 \\
\$ & 597\end{array}$ & $\begin{array}{l}49.645 \\
37.344 \\
39.738 \\
29.774\end{array}$ & $\begin{array}{l}5.363 \\
5: 363 \\
5: 363 \\
5: 363\end{array}$ \\
\hline $\begin{array}{l}\text { INSULATION } \\
\text { SPECS }\end{array}$ & INCREMENTAL & INCREMENTAL & $\begin{array}{l}\text { INCREMENTAL } \\
\text { ANNUAL HTC }\end{array}$ & $\begin{array}{l}\text { INCREMENTAL } \\
\text { ANNUAL CLG }\end{array}$ \\
\hline $\begin{array}{l}\text { B3 } \\
\text { C3 } \\
\text { EXTRA }\end{array}$ & $\begin{array}{l}.096 \\
.043 \\
.015\end{array}$ & $\begin{array}{r}101 \\
\$ 470 \\
\$ \quad 26\end{array}$ & $\begin{array}{r}12.302 \\
5.606 \\
1.964\end{array}$ & $\begin{array}{l}.000 \\
.000 \\
.000\end{array}$ \\
\hline
\end{tabular}

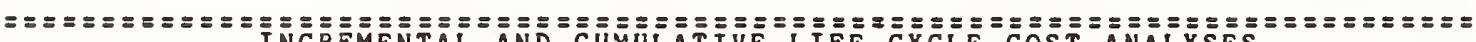
INCREMENTAL AND COUMULATIVE

GAS FURNACE
ELECTRIC AIR COND. e $\$ 220 /$ THERM

INCREMENTAL

\begin{tabular}{|c|c|c|c|c|c|c|}
\hline $\begin{array}{l}\text { INSULATION } \\
\text { SPECS }\end{array}$ & $\begin{array}{l}\text { HEATING } \\
\text { SAVINGS }\end{array}$ & $\begin{array}{l}\text { COOLING } \\
\text { SAVINGS }\end{array}$ & SÂVINGS & $\begin{array}{l}\text { INSUL. } \\
\text { COST }\end{array}$ & $\begin{array}{l}\text { NET } \\
\text { SAVINGS }\end{array}$ & $\begin{array}{l}\text { CUMULATIVE } \\
\text { NET } \\
\text { SAVINGS }\end{array}$ \\
\hline XRA & $\begin{array}{r}1262 \\
\$ \quad 575 \\
201\end{array}$ & $\$$ & $\begin{array}{r}1262 \\
\$ \quad 575 \\
\quad 201\end{array}$ & $\begin{array}{r}\$ 101 \\
\$ 470 \\
\$ \quad 26\end{array}$ & $\begin{array}{r}1161 \\
\$ \quad 105 \\
\quad 175\end{array}$ & $\begin{array}{l}1161 \\
11266 \\
\$ 1441\end{array}$ \\
\hline
\end{tabular}

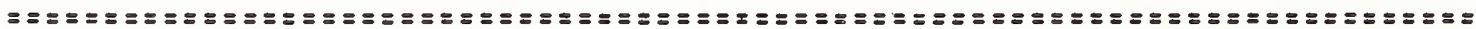

OILFURNACE
ELECTRICAIR COND. $\$ \$ 470 / G A L L O N$

INCREMENTAL

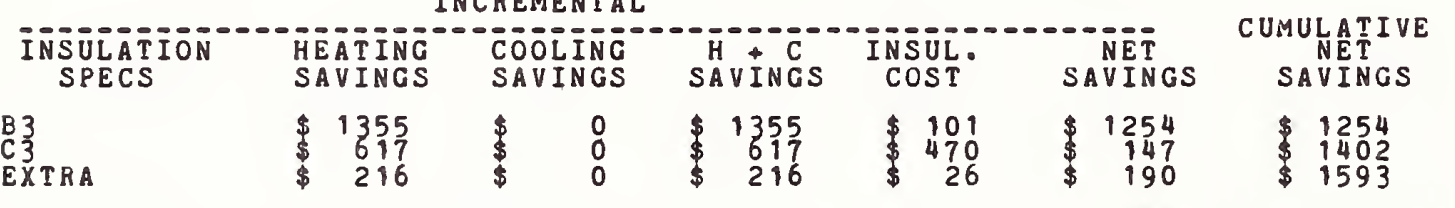

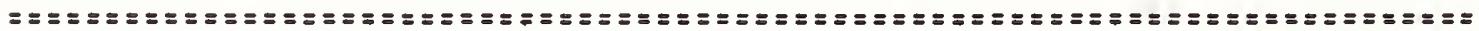

ELECTRIC FURNACE
ELECTRIC AIR COND. $e^{\$ 040 / K W H} \quad \begin{aligned} & \text { PWF } \\ & \$ 040 / K W H\end{aligned}=18.215$

INCREMENTAL

\begin{tabular}{|c|c|c|c|c|c|c|}
\hline INSULATION & $\begin{array}{l}\text { HEATING } \\
\text { SAVINGS }\end{array}$ & $\begin{array}{l}\text { COOLING } \\
\text { SAVINGS }\end{array}$ & SÄUINGS & $\begin{array}{l}\text { INSUL. } \\
\text { COST }\end{array}$ & SAVINGS & $\begin{array}{c}\text { CUMULATIVE } \\
\text { NET } \\
\text { SAVINGS }\end{array}$ \\
\hline$A$ & $\begin{array}{r}\$ 2626 \\
\$ 1196 \\
\$ 419\end{array}$ & $\$$ & $\begin{array}{r}2626 \\
\$ 1196 \\
\$ 419\end{array}$ & $\begin{array}{r}\$ 101 \\
\$ 470 \\
\$ \quad 26\end{array}$ & $\begin{array}{r}2525 \\
\$ \quad 726 \\
393\end{array}$ & $\begin{array}{r}2525 \\
3251 \\
\$ 3645\end{array}$ \\
\hline
\end{tabular}

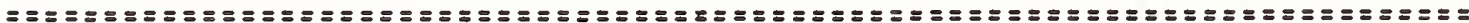
HEAT PUMP
ELECTAIC AIR COND. e $\$ 040 / \mathrm{KWH}$

INCREMENTAL

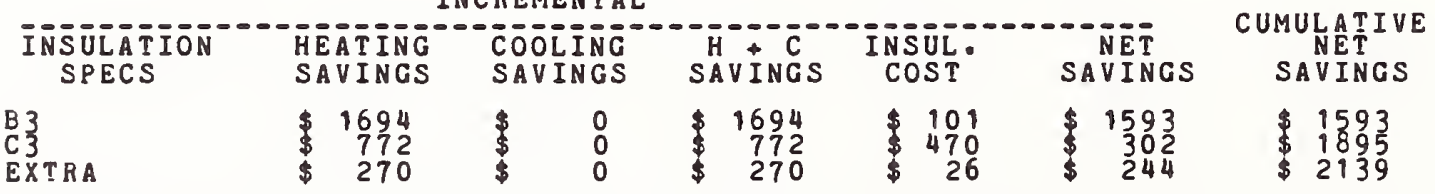

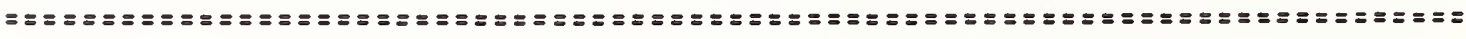


HUD 1

PHOENIX ARIZONA 200 SO FT HOUSE 8 IN BLOCK (OPEN CORE) COOLING LOADS BASED ON NO HOUSE COOLG SAVINGS

\begin{tabular}{|c|c|c|c|c|}
\hline INSULATION & VALUE & $\begin{array}{l}\text { INSUL } \\
\text { COST }\end{array}$ & $\begin{array}{l}\text { ANNUAL }{ }^{\text {HTG }} \\
\text { (MILLION BTU) }\end{array}$ & $\begin{array}{l}\text { ANNUAL CLG } \\
\text { (MILLIOiV BTU) }\end{array}$ \\
\hline $\begin{array}{l}\text { BASE CASE } \\
\text { B3 } \\
\text { C } 3 \\
\text { EXTRA }\end{array}$ & $\begin{array}{r}.249 \\
.153 \\
.110 \\
.095\end{array}$ & $\begin{array}{l}\$ 10 \\
\$ 571 \\
\$ 597\end{array}$ & $\begin{array}{l}4.035 \\
2.685 \\
2: 157 \\
1.980\end{array}$ & $\begin{array}{l}42.003 \\
42.003 \\
42.003 \\
42.003\end{array}$ \\
\hline $\begin{array}{l}\text { INSULATION } \\
\text { SPECS }\end{array}$ & INCREMENTAL & $\begin{array}{c}\text { INCREMENTAL } \\
\text { COST }\end{array}$ & $\begin{array}{l}\text { INCREMENTAL } \\
\text { ANNUAL HTG }\end{array}$ & $\begin{array}{l}\text { INCREMENTAL } \\
\text { ANNUAL CLG }\end{array}$ \\
\hline $\begin{array}{l}\text { B } 3 \\
C 3 \\
\text { EXTRA }\end{array}$ & $\begin{array}{l}.096 \\
.043 \\
.015\end{array}$ & $\begin{array}{r}101 \\
\$ 470 \\
\$ \quad 26\end{array}$ & $\begin{array}{r}1.349 \\
: 528 \\
.177\end{array}$ & $\begin{array}{l}.000 \\
.000 \\
.000\end{array}$ \\
\hline
\end{tabular}

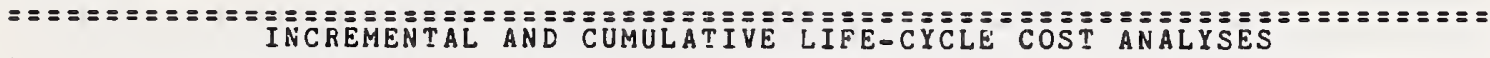

GAS FURNACE
ELECTRICAIR COND. $\$ 220 /$ THERM $\$ P W F=29.017$ INCRENENTAL

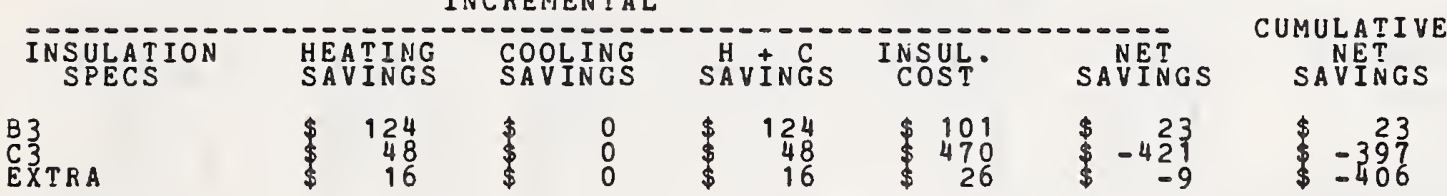

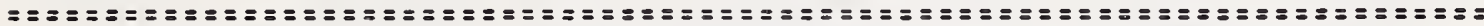
OILFURNACE
ELECTKICAIR COND. e $\$ 510 / G A L L O N:$ PWF $=24.167$
PWF $=20.306$

INCREMENTAL

\begin{tabular}{|c|c|c|c|c|c|c|c|c|c|c|}
\hline $\begin{array}{l}\text { NSULATION } \\
\text { SPECS. }\end{array}$ & & $\begin{array}{l}\text { TING } \\
\text { INGS }\end{array}$ & & $\begin{array}{l}\text { ING } \\
\text { IGS }\end{array}$ & & $\stackrel{+}{\mathrm{C}} \mathrm{G}$ S & $\begin{array}{l}\text { INSUL. } \\
\text { COST }\end{array}$ & & VET & $\begin{array}{c}\text { NET } \\
\text { SAVINGS }\end{array}$ \\
\hline 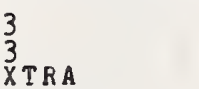 & $\begin{array}{l}\$ \\
\$\end{array}$ & $\begin{array}{r}152 \\
59 \\
20\end{array}$ & $\begin{array}{l}\$ \\
\$ \\
\$\end{array}$ & $\begin{array}{l}0 \\
0 \\
0\end{array}$ & $\begin{array}{l}\$ \\
\$ \\
\$\end{array}$ & $\begin{array}{r}152 \\
59 \\
20\end{array}$ & $\begin{array}{rr} & 101 \\
\$ & 470 \\
\$ & 26\end{array}$ & $\$$ & $\begin{array}{r}51 \\
-410 \\
-5\end{array}$ & $\begin{array}{r}51 \\
\$-359 \\
\$-365\end{array}$ \\
\hline
\end{tabular}

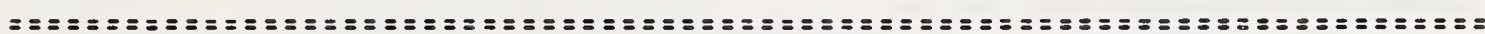

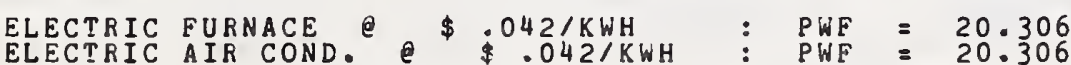

IN CREMENTAL

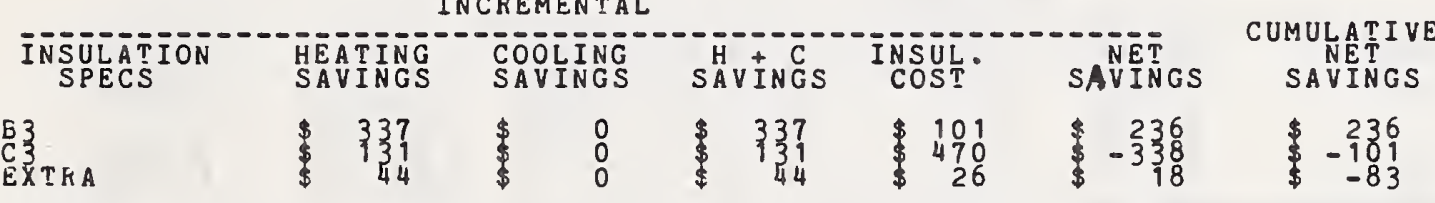

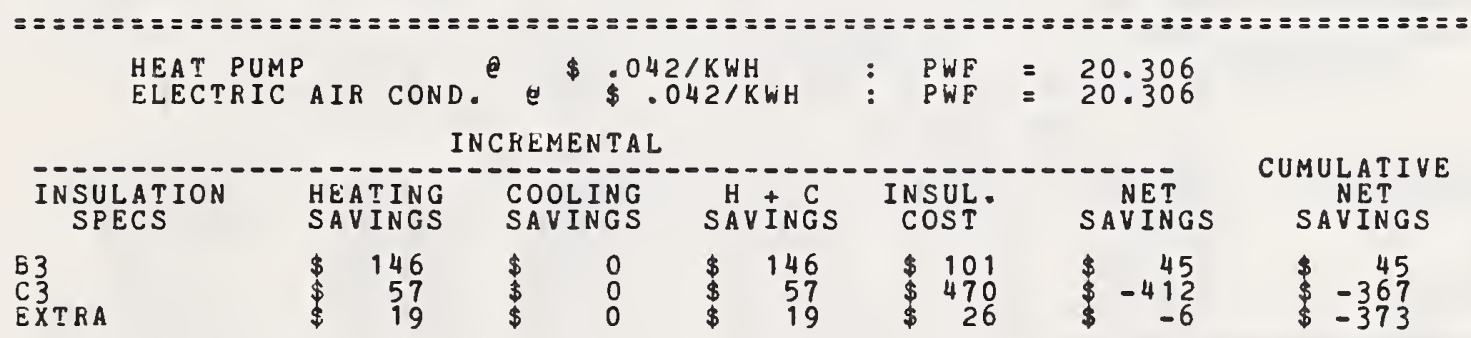

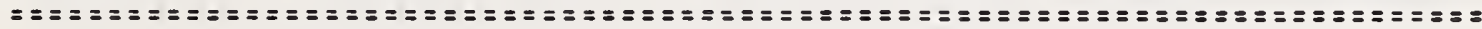


HUD 1

TAMPA FLORIDA 200 SO FT HOUSE 8 IN BLOCK (OPEN CORE) COOLING LOADS BASED ON NO COOLING SAVINGS

\begin{tabular}{|c|c|c|c|c|}
\hline $\begin{array}{l}\text { INSULATION } \\
\text { SPECS }\end{array}$ & $\stackrel{U}{\text { VALUE }}$ & $\begin{array}{l}\text { INSUL } \\
\text { COST }\end{array}$ & $\begin{array}{l}\text { ANNUAL HTG } \\
\text { (MILLION ETU) }\end{array}$ & $\begin{array}{l}\text { ANNUAL CLG } \\
\text { (MILLION ETU) }\end{array}$ \\
\hline $\begin{array}{l}\text { EASE CASE } \\
\text { E } \\
C \overline{3} \\
\text { EXTRA }\end{array}$ & $\begin{array}{r}.240 \\
.153 \\
.190 \\
.095\end{array}$ & $\begin{array}{ll}\$ & c \\
\$ & 101 \\
\$ & 571 \\
\$ & 597\end{array}$ & $\begin{array}{r}.950 \\
.505 \\
.583 \\
.343\end{array}$ & $\begin{array}{l}38 \cdot 162 \\
30: 162 \\
3850162 \\
38: 152\end{array}$ \\
\hline $\begin{array}{l}\text { IlISULATION } \\
\text { SPECS }\end{array}$ & INCREMENTAL & $\begin{array}{c}\text { INCREMENTAL } \\
\text { COST }\end{array}$ & $\begin{array}{l}\text { INCREMENTAL } \\
\text { ANNUAL HTG }\end{array}$ & $\begin{array}{l}\text { INCKEMENTAL } \\
\text { ANNUAL CLG }\end{array}$ \\
\hline $\begin{array}{l}\text { B3 } \\
\text { C3 } \\
\text { EXTRA }\end{array}$ & $\begin{array}{l}.096 \\
.043 \\
.015\end{array}$ & $\begin{array}{rr}\$ 101 \\
\$ 470 \\
\$ \quad 26\end{array}$ & $\begin{array}{r}.345 \\
.122 \\
.040\end{array}$ & $\begin{array}{l}.000 \\
.000 \\
.000\end{array}$ \\
\hline
\end{tabular}

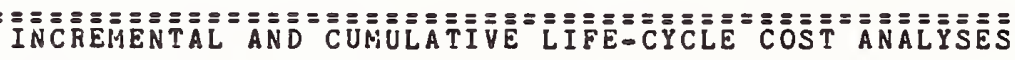

GAS FURNACE
ELECTRICAIR COND. $e^{\$ 210 / T H E R M}: \$ P$ PWF

INCREMENTAL

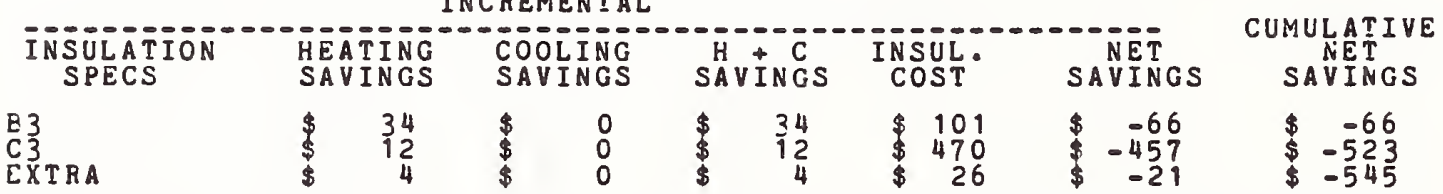

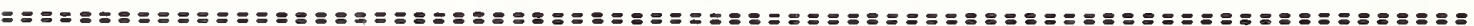

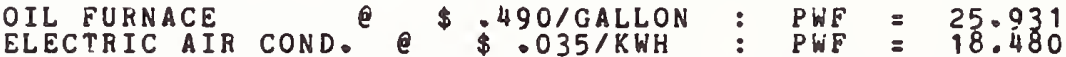

INCREMENTAL

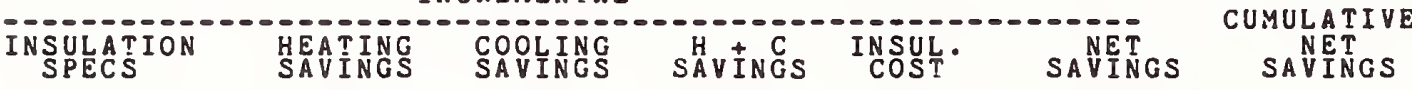

$\begin{array}{lllllllllll}\text { B3 } & \$ & 40 & \$ & 0 & \$ & 40 & \$ 101 & \$-60 & \$ 60 \\ C 3 T \text { ERA } & \$ & 14 & \$ & 0 & \$ & 14 & \$ 479 & -455 & -516 \\ \text { EIT } & \$ & 4 & \$ & 0 & \$ & 4 & \$ 26 & \$-27 & \$-538\end{array}$

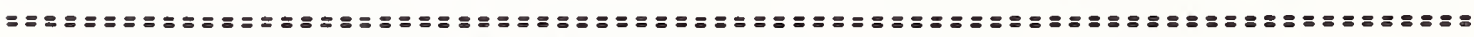

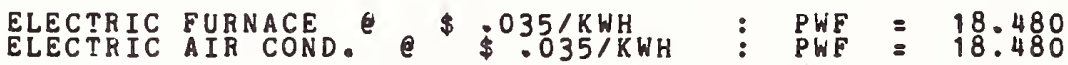

INCREMENTAL

\begin{tabular}{|c|c|c|c|c|c|c|c|c|}
\hline $\begin{array}{l}\text { INSULATION } \\
\text { SPECS }\end{array}$ & & $\begin{array}{l}I I N G \\
\text { INGS }\end{array}$ & & $\begin{array}{l}\text { NG } \\
\text { GS }\end{array}$ & $\begin{array}{l}\text { HAV C } \\
\text { SAV ING }\end{array}$ & $\begin{array}{l}\text { INSUL: } \\
\text { COST }\end{array}$ & SAVINGS & $\begin{array}{c}\text { CUMULATIVE } \\
\text { SAVINGS }\end{array}$ \\
\hline$T R A$ & $\$$ & $\begin{array}{l}65 \\
23 \\
7\end{array}$ & $\$$ & $\begin{array}{l}0 \\
0 \\
0\end{array}$ & $\begin{array}{r}65 \\
23 \\
7\end{array}$ & $\begin{array}{r}101 \\
470 \\
26\end{array}$ & $\begin{array}{r}-35 \\
-446 \\
-18\end{array}$ & $\begin{array}{l}-385 \\
-482 \\
-500\end{array}$ \\
\hline
\end{tabular}

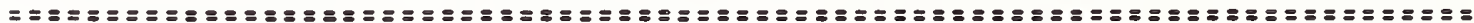
HEAT PUMP
ELECTRIC AIR COND. INCREMENTAL

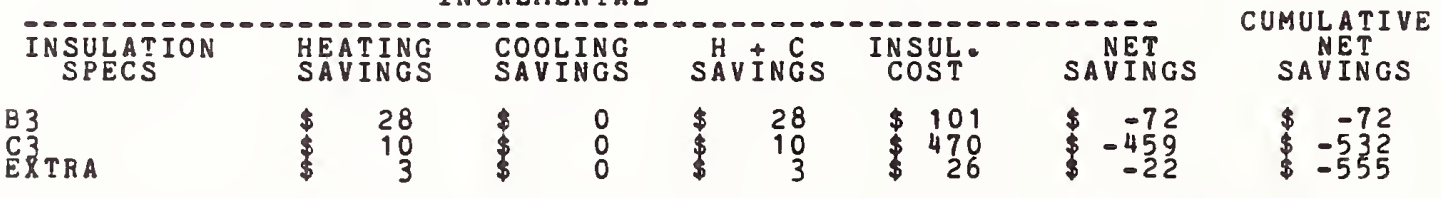

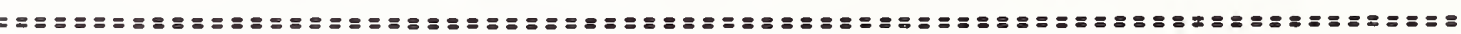


HUD 1

ASHINGTON D.C ${ }_{1200 \text { SO FT HOUSE }} 8$ IN BLOCK (OPEN CORE) COOLING LOADS BASED ON NO COOLING SAVINGS

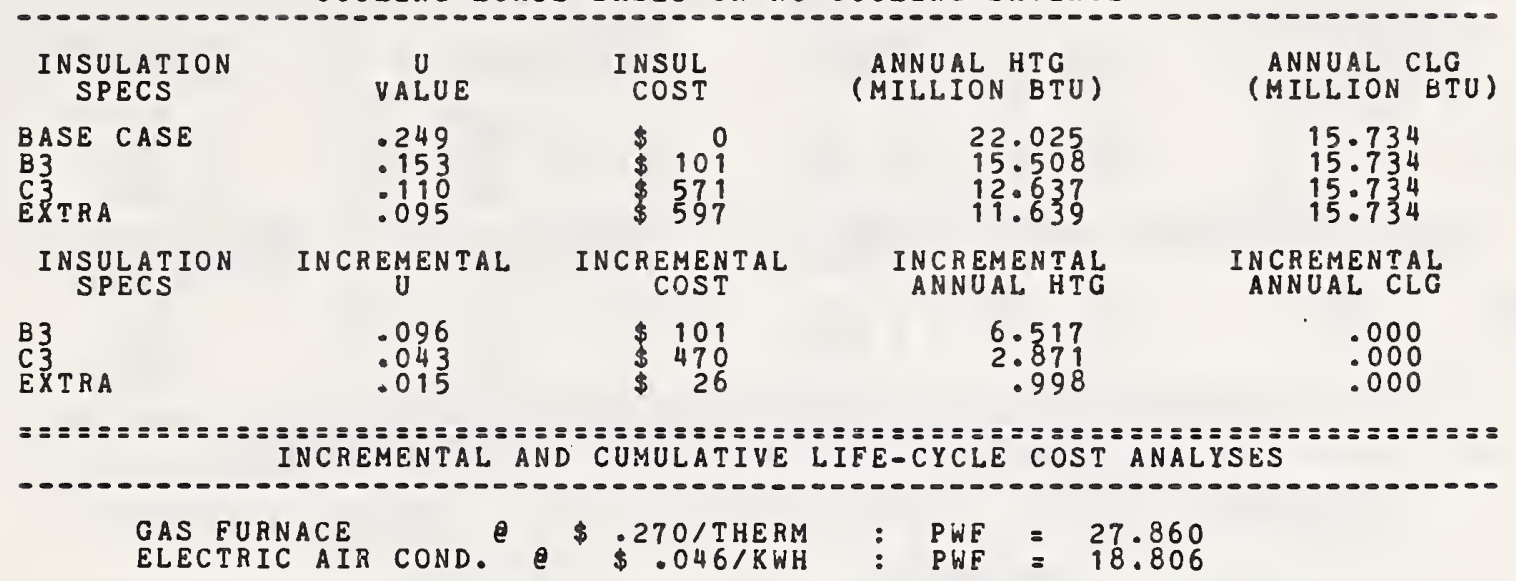

INCREMENTAL

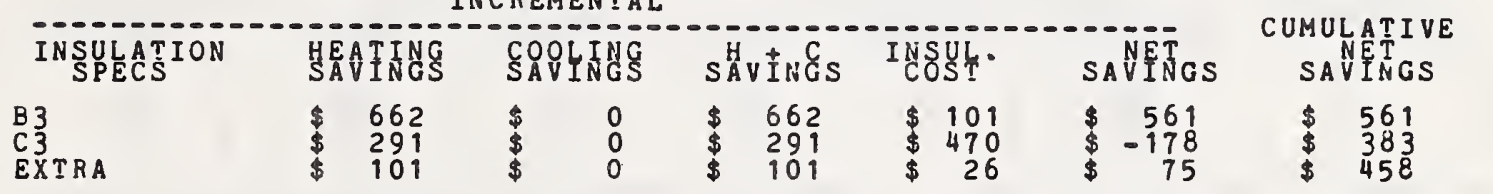

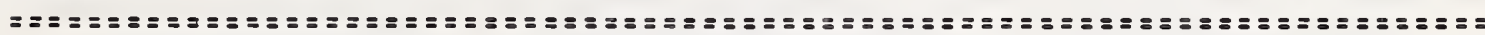

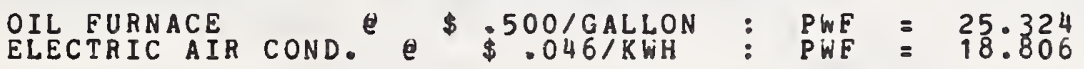

INCREMENTAL

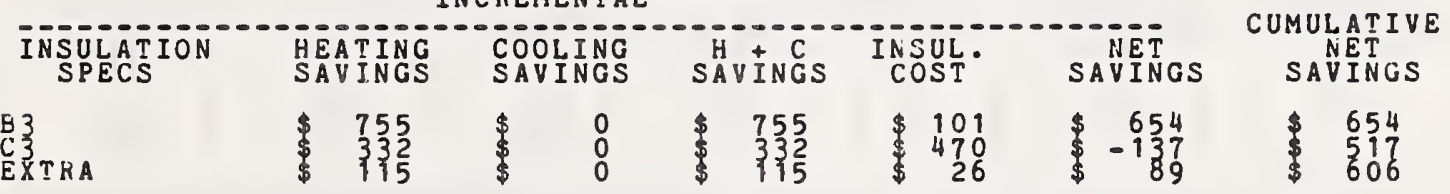

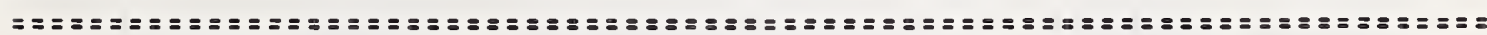

ELECTRIC FURNACE e $\$ .046 / K W H$
ELECTRIC AIR COND.

INCREMENTAL

\begin{tabular}{|c|c|c|c|c|c|c|}
\hline $\begin{array}{l}\text { INSULATION } \\
\text { SPECS }\end{array}$ & $\begin{array}{l}\text { HEATING } \\
\text { SAVINGS }\end{array}$ & $\begin{array}{l}\text { COOLING } \\
\text { SAVINGS }\end{array}$ & SÄVİNGS & $\begin{array}{l}\text { INSUL. } \\
\text { COST }\end{array}$ & SAVINGS & $\begin{array}{l}\text { NET } \\
\text { SAVINGS }\end{array}$ \\
\hline $\begin{array}{l}3 \\
3 \\
\text { ETRA }\end{array}$ & $\begin{array}{r}1651 \\
\$ \quad 727 \\
\$ \quad 253\end{array}$ & $\$$ & $\begin{array}{r}1651 \\
\$ \quad 727 \\
\$ \quad 253\end{array}$ & $\begin{array}{r}101 \\
\$ 470 \\
\$ \quad 26\end{array}$ & $\begin{array}{r}1550 \\
257 \\
227\end{array}$ & $\begin{array}{r}1550 \\
\$ \quad 1808 \\
\$ 2035\end{array}$ \\
\hline
\end{tabular}

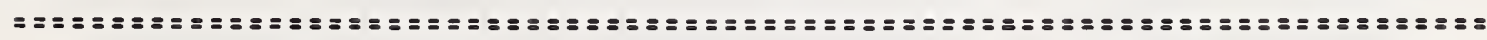
HEAT PUMP
ELECTRIC AIR COND. e $\$ 046 / K W H$

INCREMENTAL

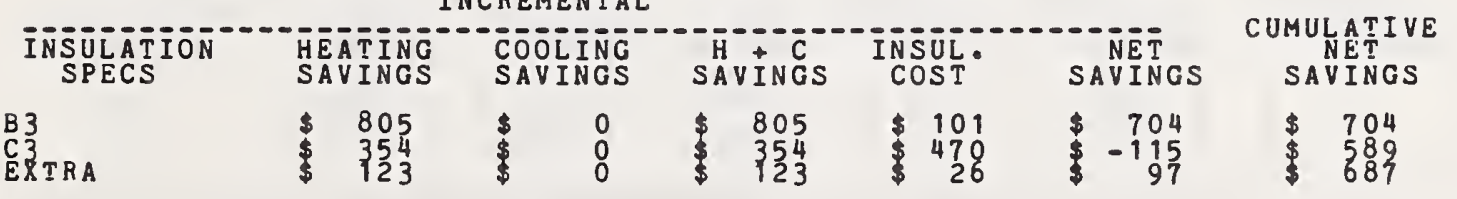

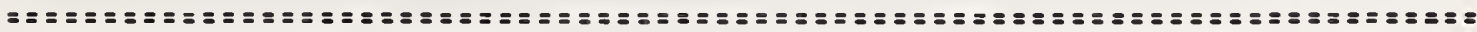


HUD2

ALBUQUERQUE NEW MEXICO

1200 SQ FT HOUSE 8

8 IN BLOCK (OPEN CORE) COOLING LOADS BASED ON NO COOLING SAVINGS

\begin{tabular}{|c|c|c|c|c|}
\hline $\begin{array}{l}\text { INSULATION } \\
\text { SPECS }\end{array}$ & $\stackrel{U}{\text { VALUE }}$ & $\begin{array}{l}\text { INSUL } \\
\text { COST }\end{array}$ & $\begin{array}{l}\text { ANNUAL HTG } \\
\text { (MILLION BTU) }\end{array}$ & $\begin{array}{l}\text { ANNUAL CLG } \\
\text { (MILLION BTU) }\end{array}$ \\
\hline $\begin{array}{l}\text { CASE CASE } \\
\text { E3 } \\
\text { D3 } \\
\text { F2 }\end{array}$ & $\begin{array}{r}.249 \\
.153 \\
.143 \\
.09\end{array}$ & $\begin{array}{r}\$ 0 \\
101 \\
370 \\
538\end{array}$ & $\begin{array}{l}22.384 \\
15.873 \\
15.197 \\
11.761\end{array}$ & $\begin{array}{l}13.006 \\
13.006 \\
13.006 \\
13.006\end{array}$ \\
\hline $\begin{array}{l}\text { INSULATION } \\
\text { SPECS }\end{array}$ & INCREMENTAL & $\begin{array}{c}\text { INCREMENTAL } \\
\text { COST }\end{array}$ & $\begin{array}{l}\text { INCREMENTAL } \\
\text { ANNUAL HTG }\end{array}$ & $\begin{array}{l}\text { INCREMENTAL } \\
\text { ANNUAL CLG }\end{array}$ \\
\hline $\begin{array}{l}\text { E3 } \\
\text { D3 } \\
\text { F2 }\end{array}$ & $\begin{array}{l}.096 \\
.010 \\
.052\end{array}$ & $\begin{array}{l}\$ 101 \\
\$ 269 \\
\$ 168\end{array}$ & $\begin{array}{r}6.511 \\
.676 \\
3.436\end{array}$ & $\begin{array}{l}.000 \\
.000 \\
.000\end{array}$ \\
\hline
\end{tabular}

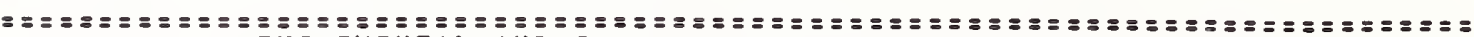
INCKEMENTAL AND CUMULATIVE LIEE-CYCLE COST ANALYSES

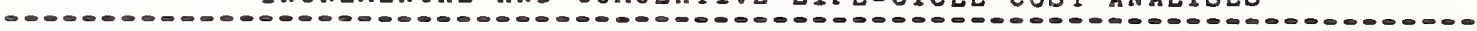

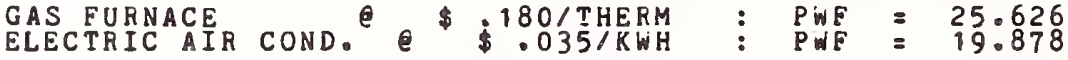

INCREMENTAL

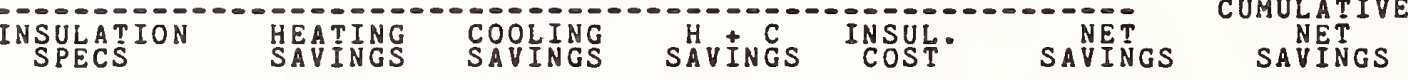
E3
D
F
395
$\$ \quad 49$
$\$ 208$
$\$ \begin{array}{ll}\$ & 0 \\ \$ & 0\end{array}$
395
$\$ 41$
$\$ 208$
101
269
$\$ 168$
294
-227
40
$\begin{array}{rr}\$ 294 \\ \$ & 66 \\ \$ & 106\end{array}$

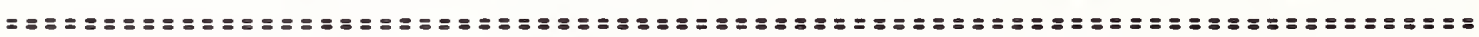

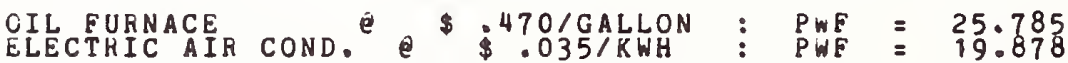

INCREMENTAL

\begin{tabular}{|c|c|c|c|c|c|c|}
\hline $\begin{array}{l}\text { INSULATION } \\
\text { SPECS }\end{array}$ & $\begin{array}{l}\text { HEATING } \\
\text { SAVINGS }\end{array}$ & $\begin{array}{l}\text { COOLING } \\
\text { SAVINGS }\end{array}$ & SAVINGS & $\begin{array}{l}\text { INSUL. } \\
\text { COST }\end{array}$ & SAVINGS & $\begin{array}{c}\text { CUMULATIVE } \\
\text { NET } \\
\text { SAVINGS }\end{array}$ \\
\hline & $\begin{array}{l}722 \\
74 \\
381\end{array}$ & $\$$ & $\begin{array}{l}722 \\
\$ 74 \\
381\end{array}$ & $\begin{array}{l}101 \\
\$ 269 \\
168\end{array}$ & $\begin{array}{r}621 \\
\$-194 \\
213\end{array}$ & $\begin{array}{l}\quad 62 \\
42 \\
\$ \\
\$ 24\end{array}$ \\
\hline
\end{tabular}

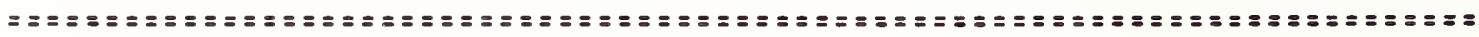

ELECTRIC FURAAE e $\$ 035 / K W H$
ELECTRIC AIR COND. $\$$ PWF $=19.878$

INCREMENTAL

\begin{tabular}{|c|c|c|c|c|c|c|}
\hline $\begin{array}{l}\text { INSULATION } \\
\text { SPECS }\end{array}$ & $\begin{array}{l}\text { HEATING } \\
\text { SAVINGS }\end{array}$ & $\begin{array}{l}\text { COOLING } \\
\text { SAVINGS }\end{array}$ & SAHVINGS & INSULT. & SAVITES & $\begin{array}{c}\text { CUMULATIVE } \\
\text { SAVETESS }\end{array}$ \\
\hline & $\begin{array}{rr}\$ 327 \\
\$ \quad 137\end{array}$ & $\$$ & $\begin{array}{rr} & 1327 \\
\$ & 137 \\
\$ & 706\end{array}$ & $\begin{array}{l}\$ 101 \\
\$ 269 \\
\$ 168\end{array}$ & $\begin{array}{r}1226 \\
\$-131 \\
\$ \\
\$\end{array}$ & $\begin{array}{l}\$ 1226 \\
\$ 1095 \\
\$ 1627\end{array}$ \\
\hline
\end{tabular}

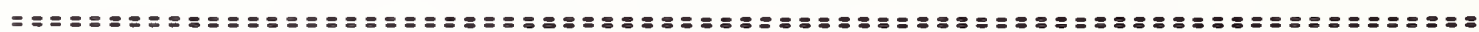
HEAT PUMP
ELECTRIC AIR COND。 e $\$ 035 / K W H$

INCREMENTAL

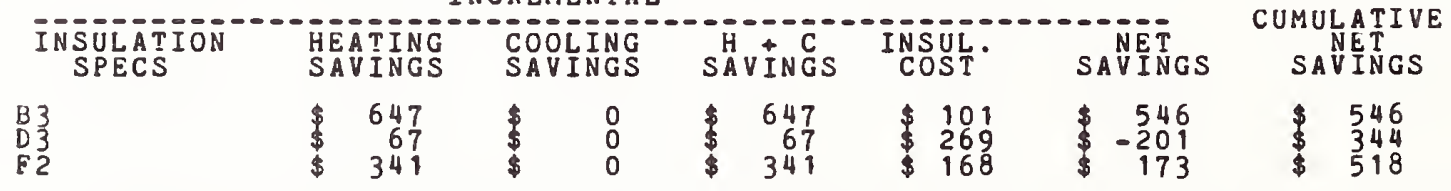


HUD 2

ATLANTA GEORGIA 8 IN BLOCK (OPEN CORE) COOLING LOADS BZ⿱ZSEDSON NOHOUSEING SAVINGS

\begin{tabular}{|c|c|c|c|c|}
\hline $\begin{array}{l}\text { INSULATION } \\
\text { SPECS }\end{array}$ & UALUE & $\begin{array}{l}\text { INSUL } \\
\text { COST }\end{array}$ & $\begin{array}{l}\text { ANNUAL HTG } \\
\text { (MILLION BTU) }\end{array}$ & $\begin{array}{l}\text { ANNUAL CLG } \\
\text { (MILLION BTU) }\end{array}$ \\
\hline $\begin{array}{l}\text { BASE CASE } \\
\text { B } 3 \\
\text { D } 3 \\
\text { H2 }\end{array}$ & $\begin{array}{l}.249 \\
: 153 \\
.143 \\
.091\end{array}$ & $\begin{array}{rr}\$ & 0 \\
\$ & 101 \\
370 \\
538\end{array}$ & $\begin{array}{l}17.124 \\
12: 929 \\
12.494 \\
10.279\end{array}$ & $\begin{array}{l}16.800 \\
16.800 \\
16.800 \\
16.800\end{array}$ \\
\hline $\begin{array}{l}\text { INSULATION } \\
\text { SPECS }\end{array}$ & INCFEMENTAL & $\begin{array}{l}\text { INCREMENTAL } \\
\text { COST }\end{array}$ & $\begin{array}{l}\text { INCREMENTAL } \\
\text { ANNUAL HTG }\end{array}$ & $\begin{array}{l}\text { INCKEMENTAL } \\
\text { ANNUAL CLC }\end{array}$ \\
\hline $\begin{array}{l}83 \\
D \overline{3} \\
F 3\end{array}$ & $\begin{array}{l}.096 \\
.010 \\
.052\end{array}$ & $\begin{array}{l}101 \\
\$ 260 \\
\$ 168\end{array}$ & $\begin{array}{r}4.195 \\
.436 \\
2.215\end{array}$ & $\begin{array}{l}.000 \\
.000 \\
.000\end{array}$ \\
\hline \multicolumn{5}{|c|}{ 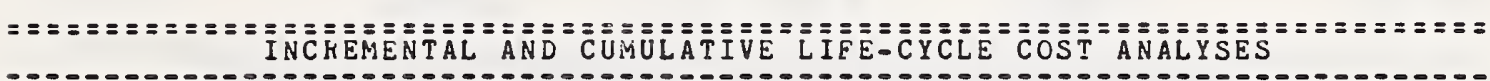 } \\
\hline
\end{tabular}

INCREMENTAL

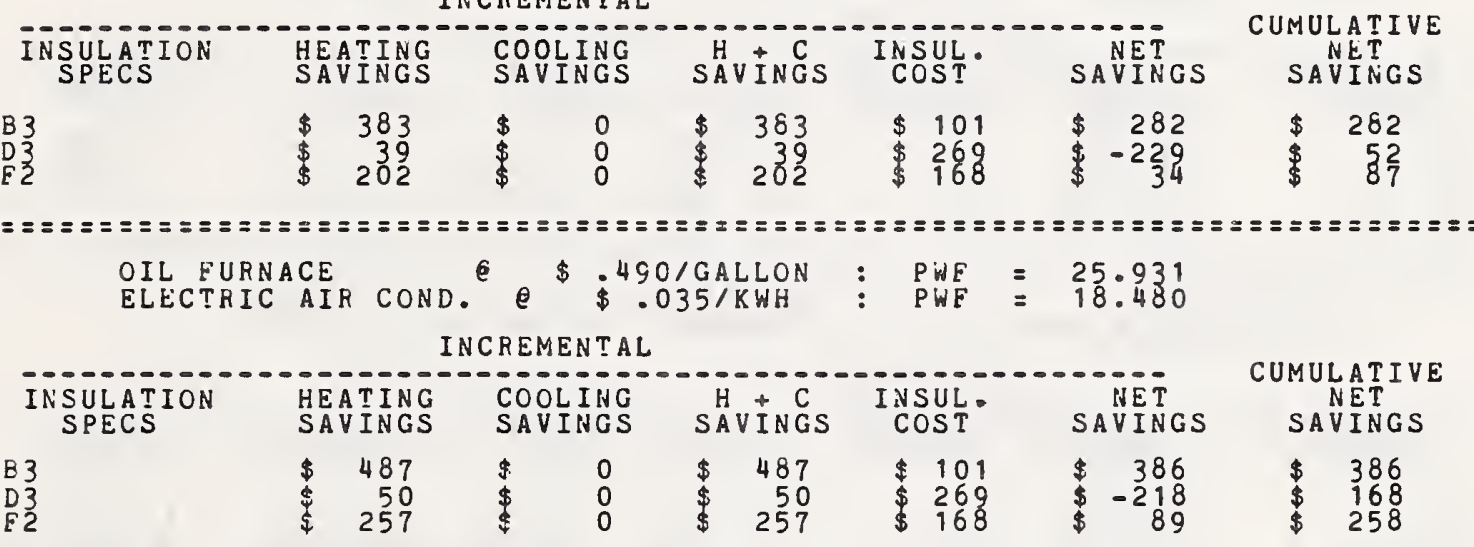

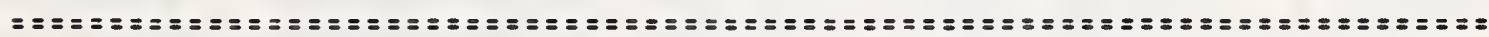

ELECTRIC FURNACE
ELECTKIC AIR COND. \$ $\$ .035 / \mathrm{KWH}$

INC REMENTAL

\begin{tabular}{|c|c|c|c|c|c|c|c|c|c|c|}
\hline $\begin{array}{l}\text { INSULAT ION } \\
\text { SPECS }\end{array}$ & & $\begin{array}{l}\text { TING } \\
\text { INGS }\end{array}$ & & & & & $\begin{array}{l}\text { INSUL } \\
\text { COST }\end{array}$ & SAVINGS & $\begin{array}{r}\mathrm{CUM} \\
\mathrm{S}\end{array}$ & $\begin{array}{l}\text { NETTE } \\
\text { VINGS }\end{array}$ \\
\hline & $\$$ & $\begin{array}{l}794 \\
82 \\
419\end{array}$ & $\$$ & $\begin{array}{l}0 \\
0 \\
0\end{array}$ & $\$$ & $\begin{array}{l}794 \\
82 \\
419\end{array}$ & $\begin{array}{l}101 \\
\$ 269 \\
\$ 168\end{array}$ & 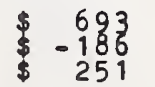 & $\$$ & $\begin{array}{l}693 \\
507 \\
759\end{array}$ \\
\hline
\end{tabular}

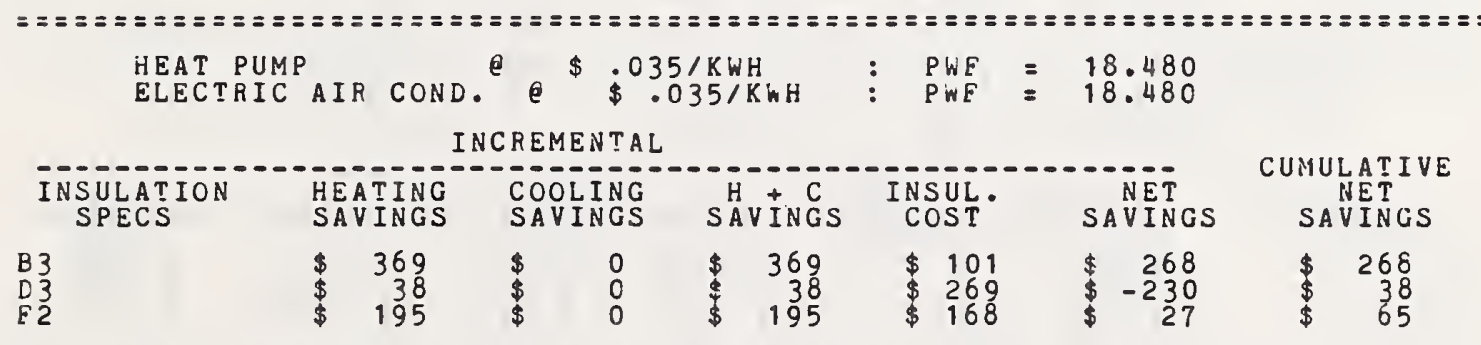

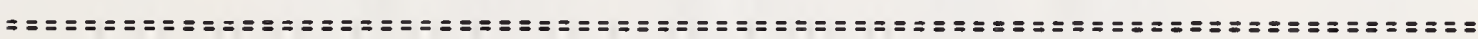




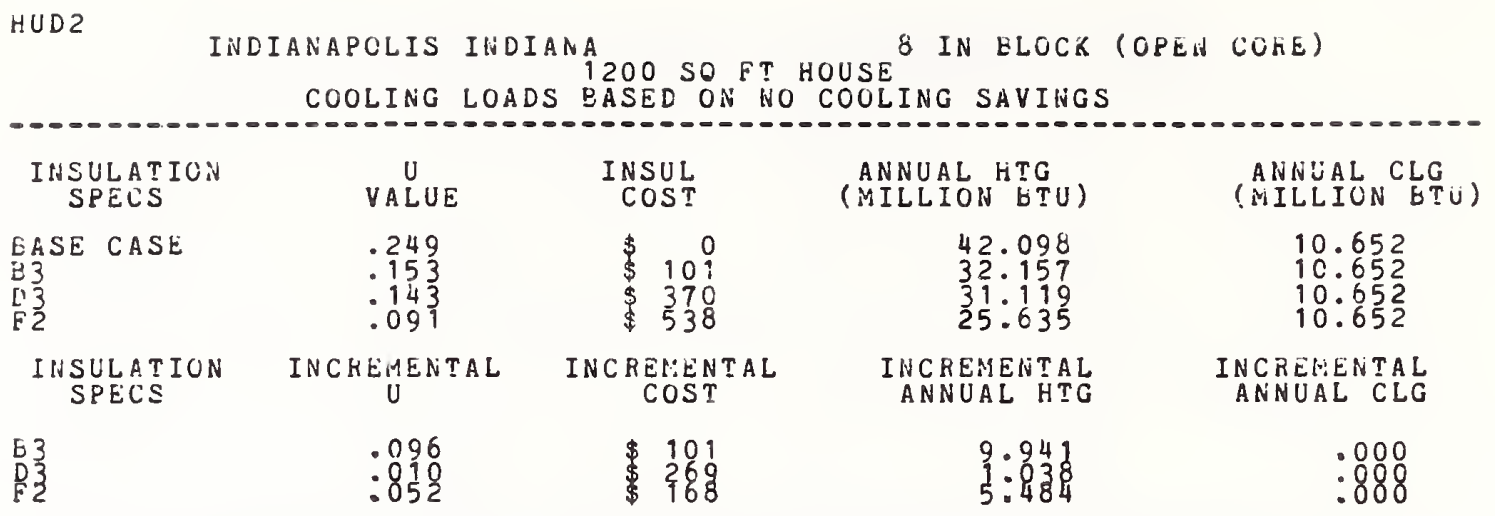

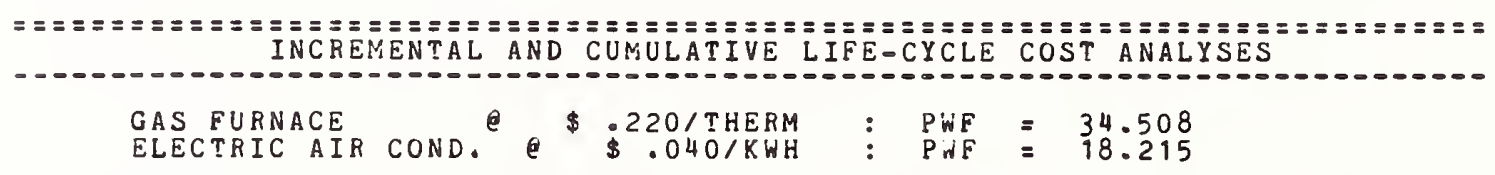
INCREMENTAL

\begin{tabular}{|c|c|c|c|c|c|c|}
\hline INSULATION & $\begin{array}{l}\text { HEATING } \\
\text { SEVINGS }\end{array}$ & COOLING & SÄVINNGS & INSUL. & SAVIETGS & SAVINGS \\
\hline $\begin{array}{l}63 \\
03\end{array}$ & $\begin{array}{rr}\$ & 1019 \\
\$ & 106 \\
\$ & 562\end{array}$ & $\begin{array}{l}\$ \\
\$\end{array}$ & $\begin{array}{rr} & 1019 \\
\$ & 106 \\
& 562\end{array}$ & $\begin{array}{l}\$ 101 \\
\$ 268 \\
\$ 168\end{array}$ & $\begin{array}{rr}\$ & 918 \\
\$ & -162 \\
\$ & 394\end{array}$ & $\begin{array}{rr} & \$ 918 \\
\$ & 1759\end{array}$ \\
\hline
\end{tabular}

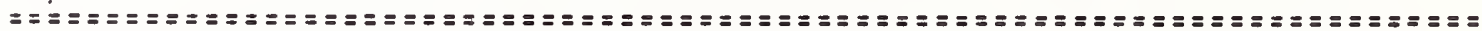

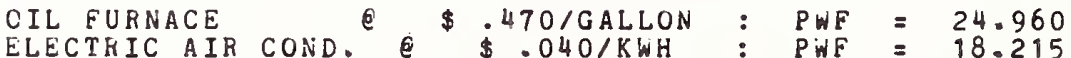

INCREMENTAL

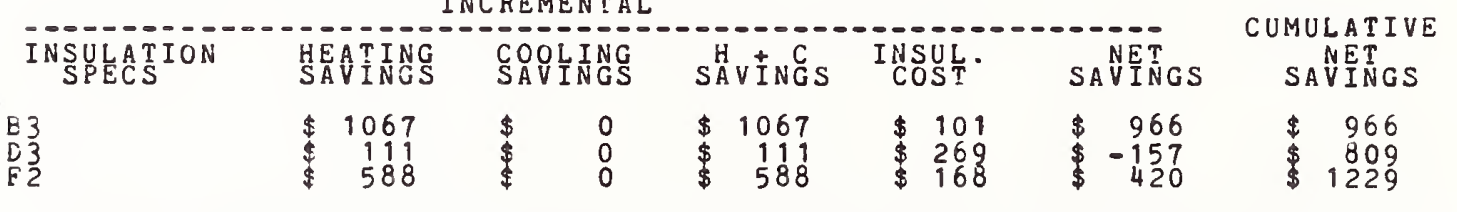

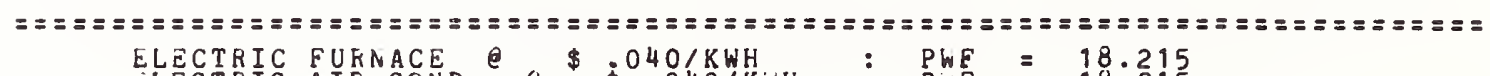

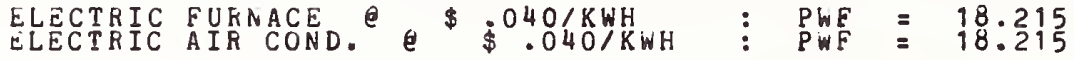

INCREMENTAL

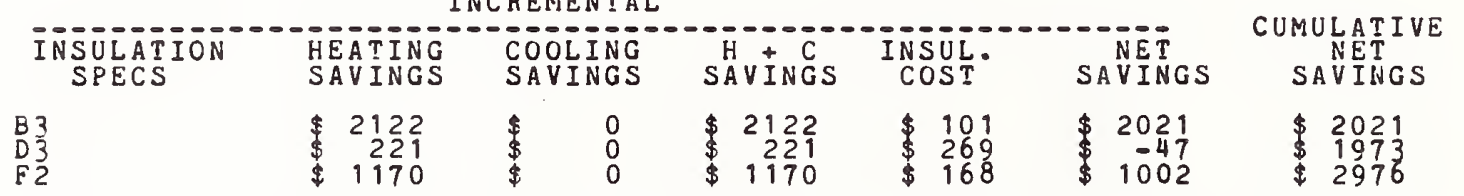

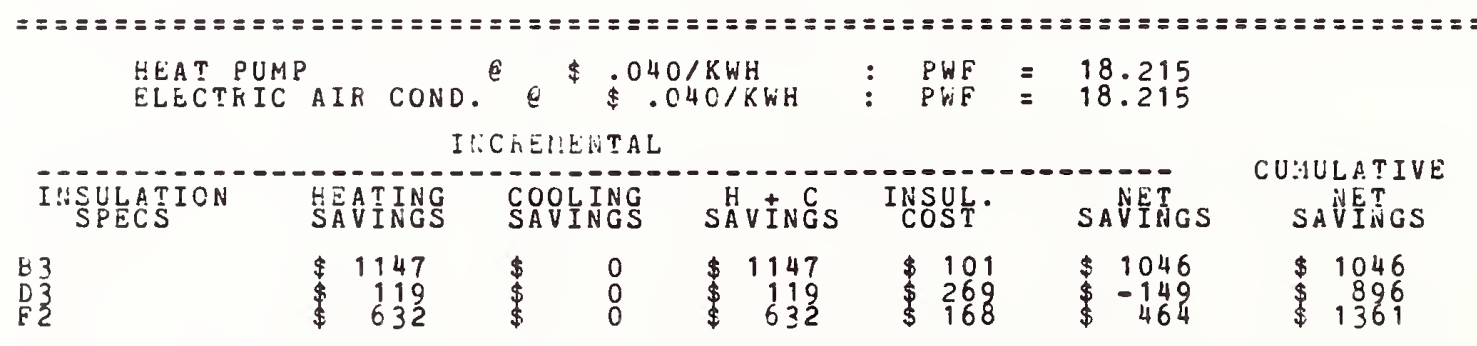

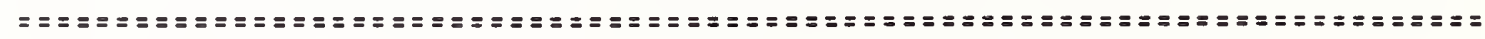


HUD 2

$$
\begin{aligned}
& \text { JACKSONVILLE FLORIDA } 1200 \text { SQ FT HOUSE IN BLOCK (OPEN COKE) } \\
& \text { COOLING LOADS BASED ON NO COOLING SAVINGS }
\end{aligned}
$$

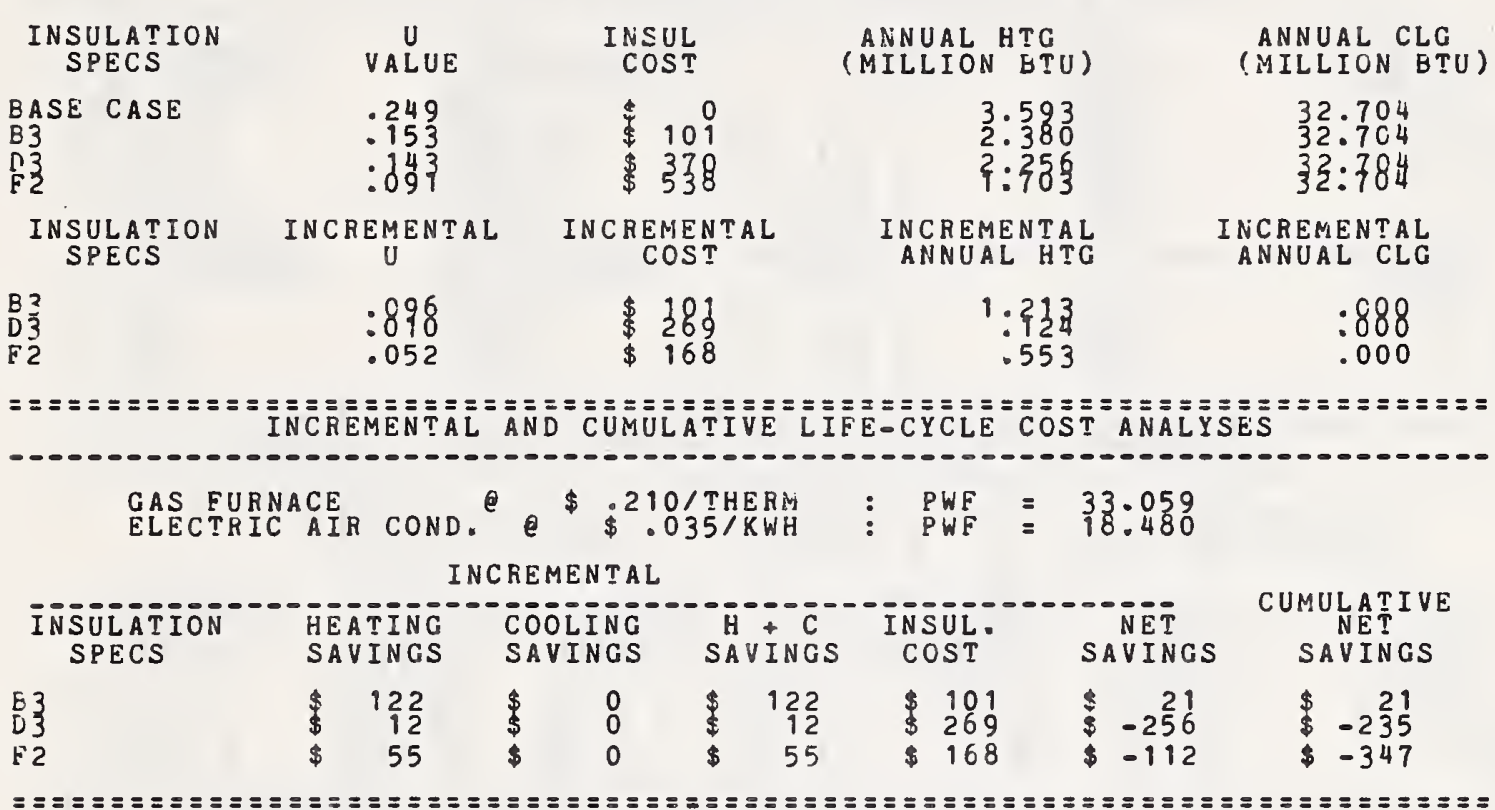

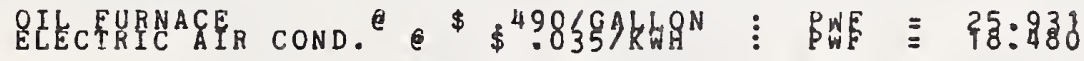

\begin{tabular}{|c|c|c|c|c|c|c|c|c|c|}
\hline $\begin{array}{l}\text { INSULATION } \\
\text { SPECS }\end{array}$ & & $\begin{array}{l}\text { TING } \\
\text { INGS }\end{array}$ & & $\begin{array}{l}\text { NG } \\
\text { GS }\end{array}$ & & $\begin{array}{l}+C^{-} \\
\text {INGS }\end{array}$ & $\begin{array}{l}\text { INSUL: } \\
\text { COST }\end{array}$ & $\begin{array}{l}\text { NET } \\
\text { SAVINGS }\end{array}$ & $\begin{array}{c}\text { CUGULAT IVE } \\
\text { NAETINGS }\end{array}$ \\
\hline & $\$$ & $\begin{array}{r}141 \\
14 \\
64\end{array}$ & $\begin{array}{l}\$ \\
\$\end{array}$ & $\begin{array}{l}0 \\
0 \\
0\end{array}$ & $\begin{array}{l}\$ \\
\$\end{array}$ & $\begin{array}{r}141 \\
14 \\
64\end{array}$ & $\begin{array}{l}101 \\
\$ 269 \\
\$ 168\end{array}$ & $\begin{array}{r}\$ 40 \\
\$-254 \\
-103\end{array}$ & $\begin{array}{r}\$ 40 \\
\$-214 \\
\$-318\end{array}$ \\
\hline
\end{tabular}

INCKEMENTAL

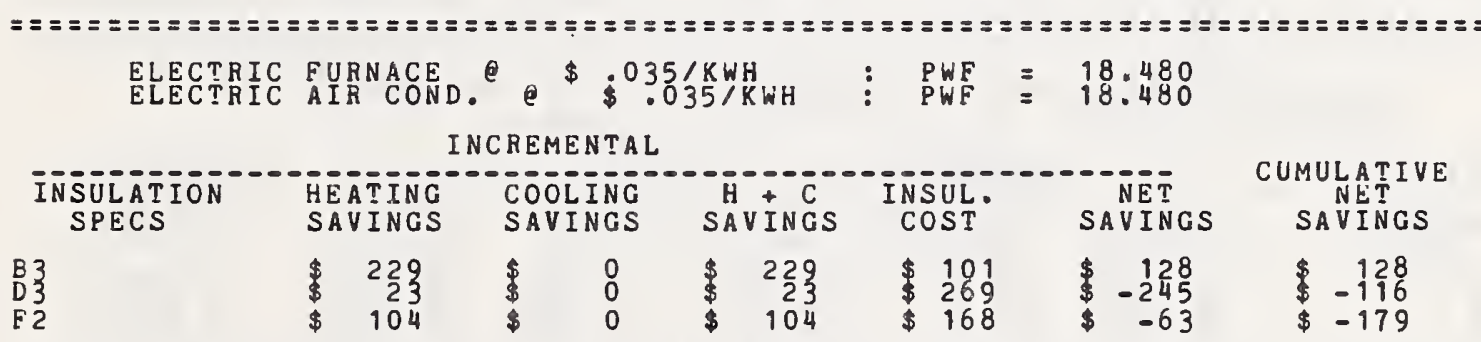

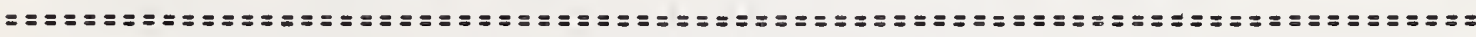
HEAT PUMP
ELECTKIC AIR COND. $e^{\$ 035 / K W H}$ \$.035/KWH INCREMENTAL

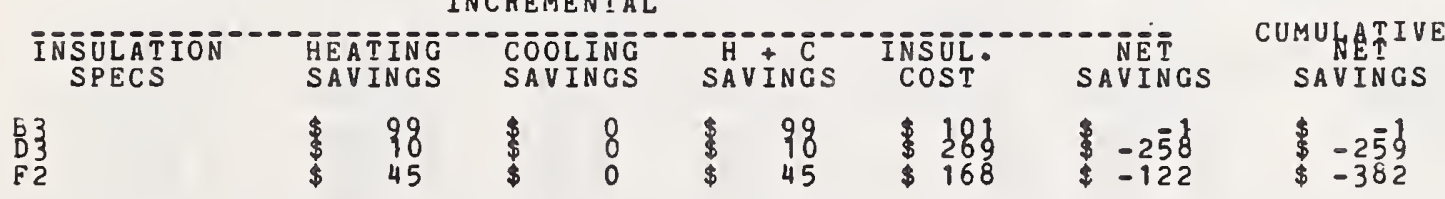


HUD2

MADISON WISCONSIN

8 IN BLOCK (OPEN CORE) COOLING LOADS BASED ON FTO HOUSE COOLING SAVINGS

\begin{tabular}{|c|c|c|c|c|}
\hline $\begin{array}{l}\text { INSULATION } \\
\text { SPECS }\end{array}$ & VALUE & $\begin{array}{l}\text { INSUL } \\
\text { COST }\end{array}$ & $\begin{array}{l}\text { ANNUAL HTG } \\
(M I L L I O N \text { BTU })\end{array}$ & $\begin{array}{l}\text { ANNUAL CLG } \\
\text { (MILLION BTU) }\end{array}$ \\
\hline $\begin{array}{l}\text { BASE CASE } \\
\text { E3 } \\
D 3 \\
\text { E2 }\end{array}$ & $\begin{array}{l}.249 \\
: 153 \\
: 143 \\
.099\end{array}$ & $\begin{array}{r}\$ 0 \\
\$ 2101 \\
\$ 370 \\
\$ 538\end{array}$ & $\begin{array}{l}49.645 \\
37: 344 \\
36: 859 \\
29.250\end{array}$ & $\begin{array}{l}5.363 \\
5: 3633 \\
5: 363 \\
5.363\end{array}$ \\
\hline $\begin{array}{l}\text { INSULATION } \\
\text { SPECS }\end{array}$ & INCREMUENTAL & $\begin{array}{c}\text { INCREMENTAL } \\
\text { COST }\end{array}$ & $\begin{array}{l}\text { INCREMENTAL } \\
\text { ANNUAL HTG }\end{array}$ & $\begin{array}{l}\text { INCREMENTAL } \\
\text { AHNGL CLG }\end{array}$ \\
\hline $\begin{array}{l}\text { E3 } \\
D 3 \\
\text { B2 }\end{array}$ & $\begin{array}{l}.096 \\
.010 \\
.052\end{array}$ & $\begin{array}{l}\$ 101 \\
\$ 268 \\
\$ 2\end{array}$ & $\begin{array}{r}12.302 \\
1: 285 \\
6: 805\end{array}$ & $\begin{array}{l}.000 \\
.888\end{array}$ \\
\hline
\end{tabular}

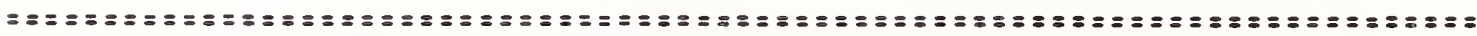
INCREMENTAL AND CUMULATIVE LIFE-CYCLE COST ANALYSES

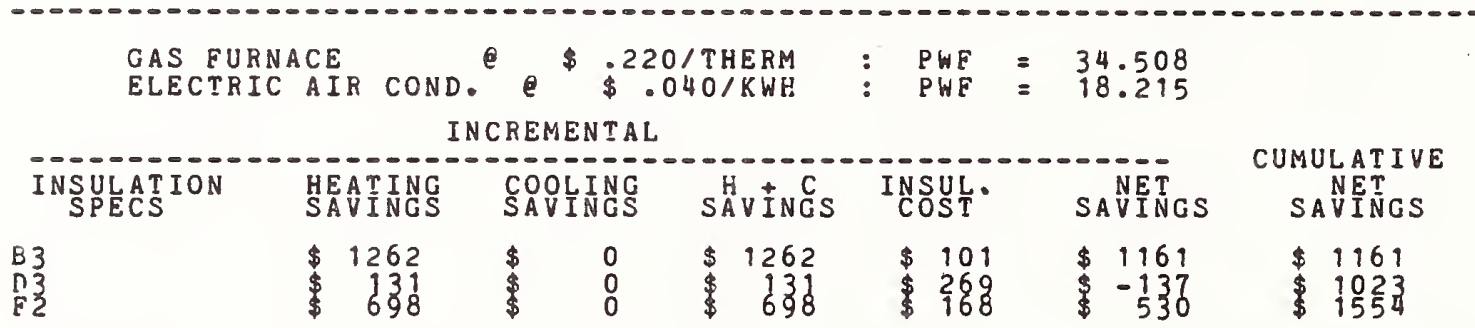

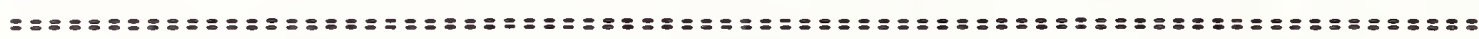

OIL FURNACE $\$$ \$ $\$ 40 / G A L L O N: P W F=24.960$

ELECTRIC AIR COND. $\$$ \$ $\$$ PWF $=18.215$

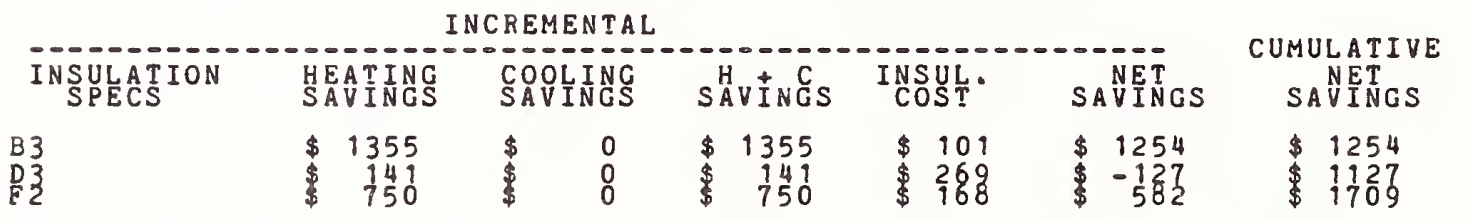

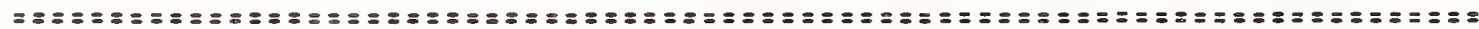

ELECTRIC FURNACE
ELECTRIC AIR COND。 $\$ \$ 040 / \mathrm{KWH}$

INCREMENTAL

\begin{tabular}{|c|c|c|c|c|c|c|}
\hline INSULEASION & 最ATIEG & SR8LING & SAVENES & I COSUT. & SAVERGS & SAVIRGS \\
\hline 3 & $\begin{array}{r}\$ 2626 \\
\$ 1274\end{array}$ & $\$$ & $\begin{array}{r}2626 \\
\$ 1274\end{array}$ & $\begin{array}{l}\$ 101 \\
\$ 269 \\
\$ 168\end{array}$ & $\begin{array}{l}2525 \\
\$ 1285\end{array}$ & $\begin{array}{l}2525 \\
\$ 2530 \\
\$ 385\end{array}$ \\
\hline
\end{tabular}

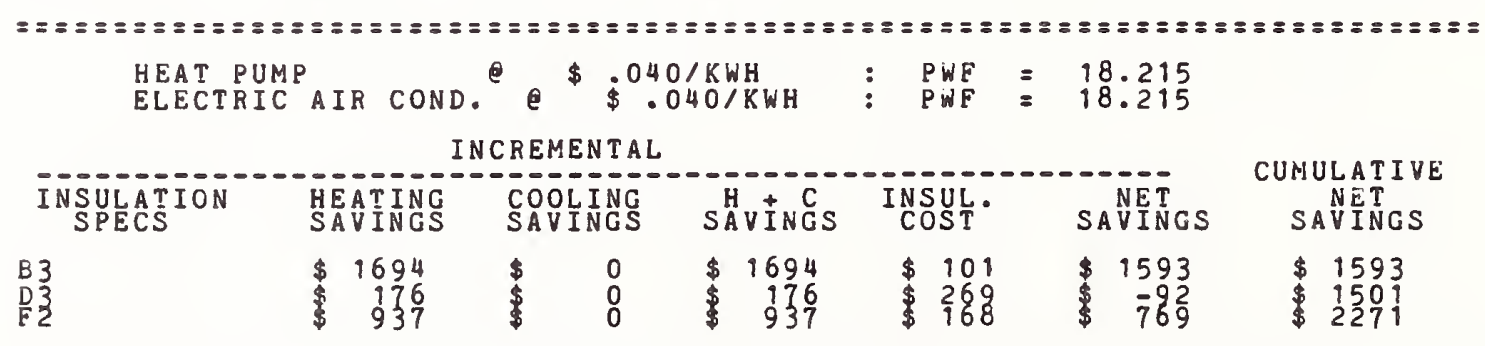




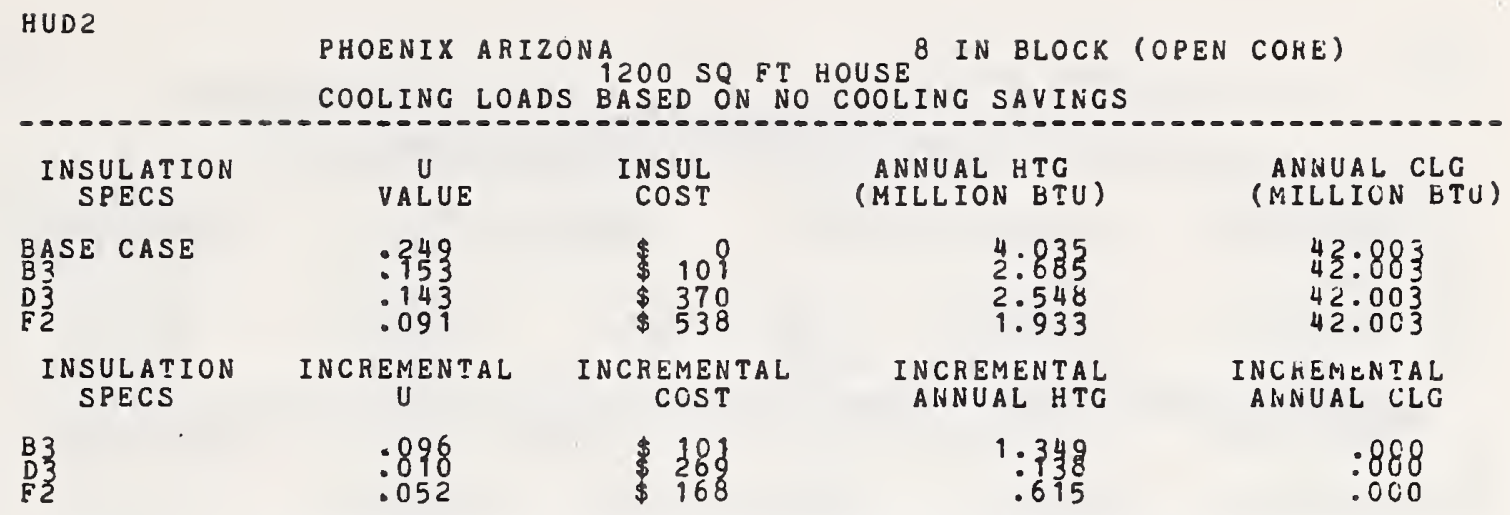

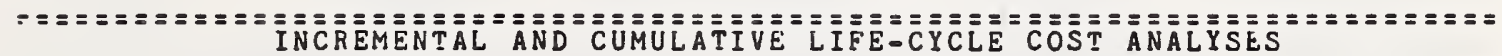

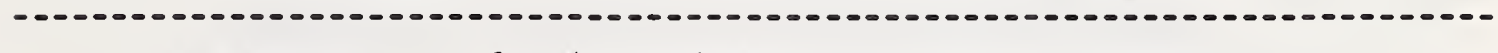
GAS FURNACE
ELECTRICAIR COND。 INCREMENTAL

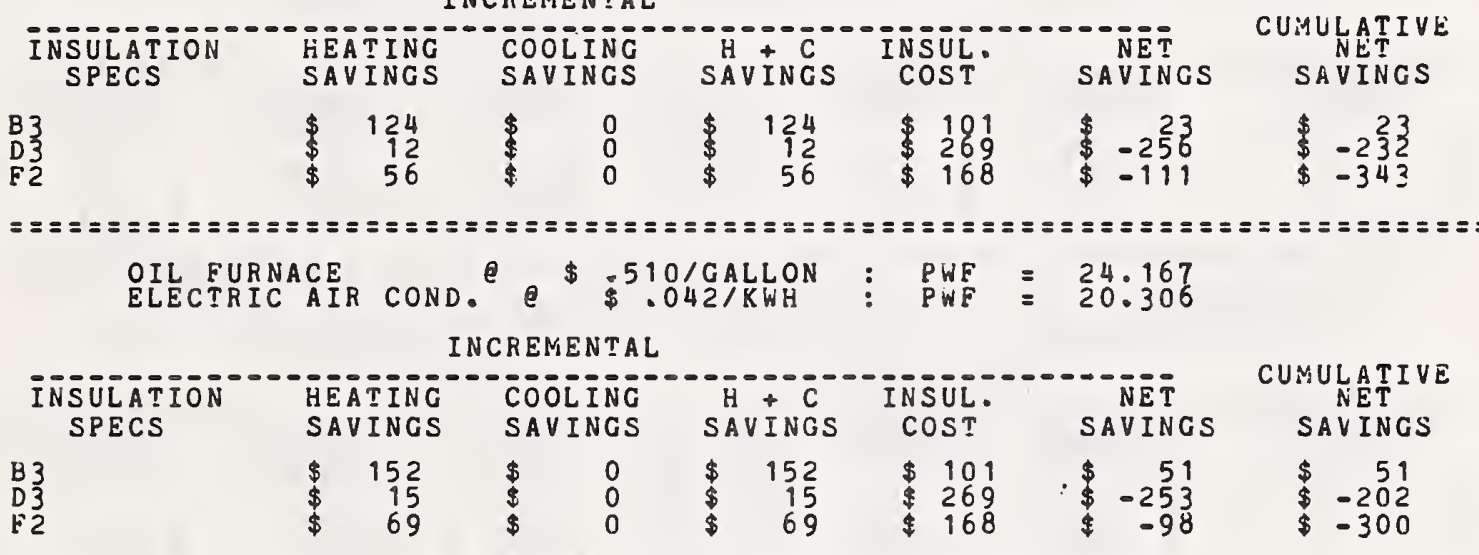

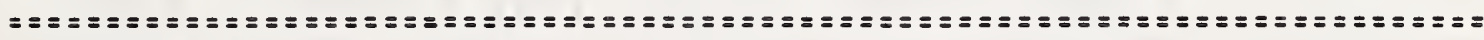

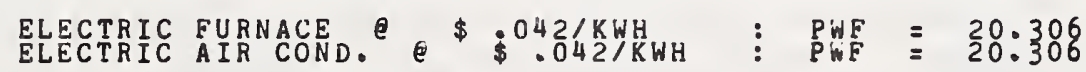
INCREMENTAL

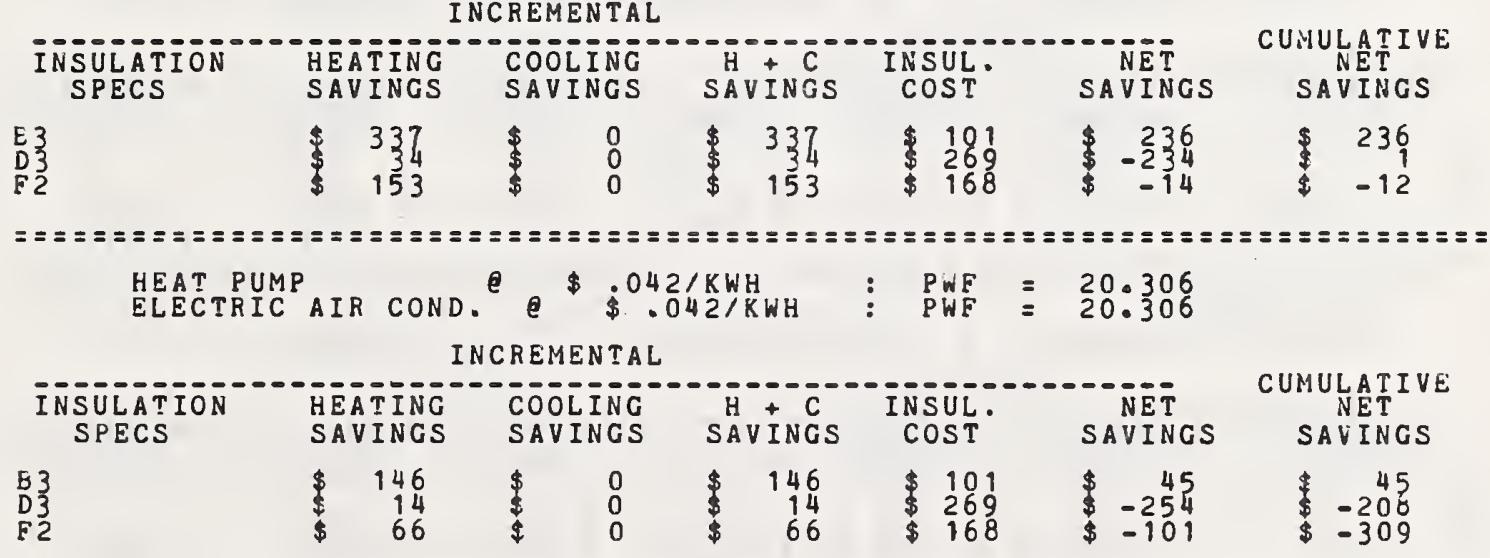


HUD 2

TAMPA FLORIDA 8 IN BLOCK (OPEN COKE) COOLING LOADS BASEDON NO COOLING SAVINGS

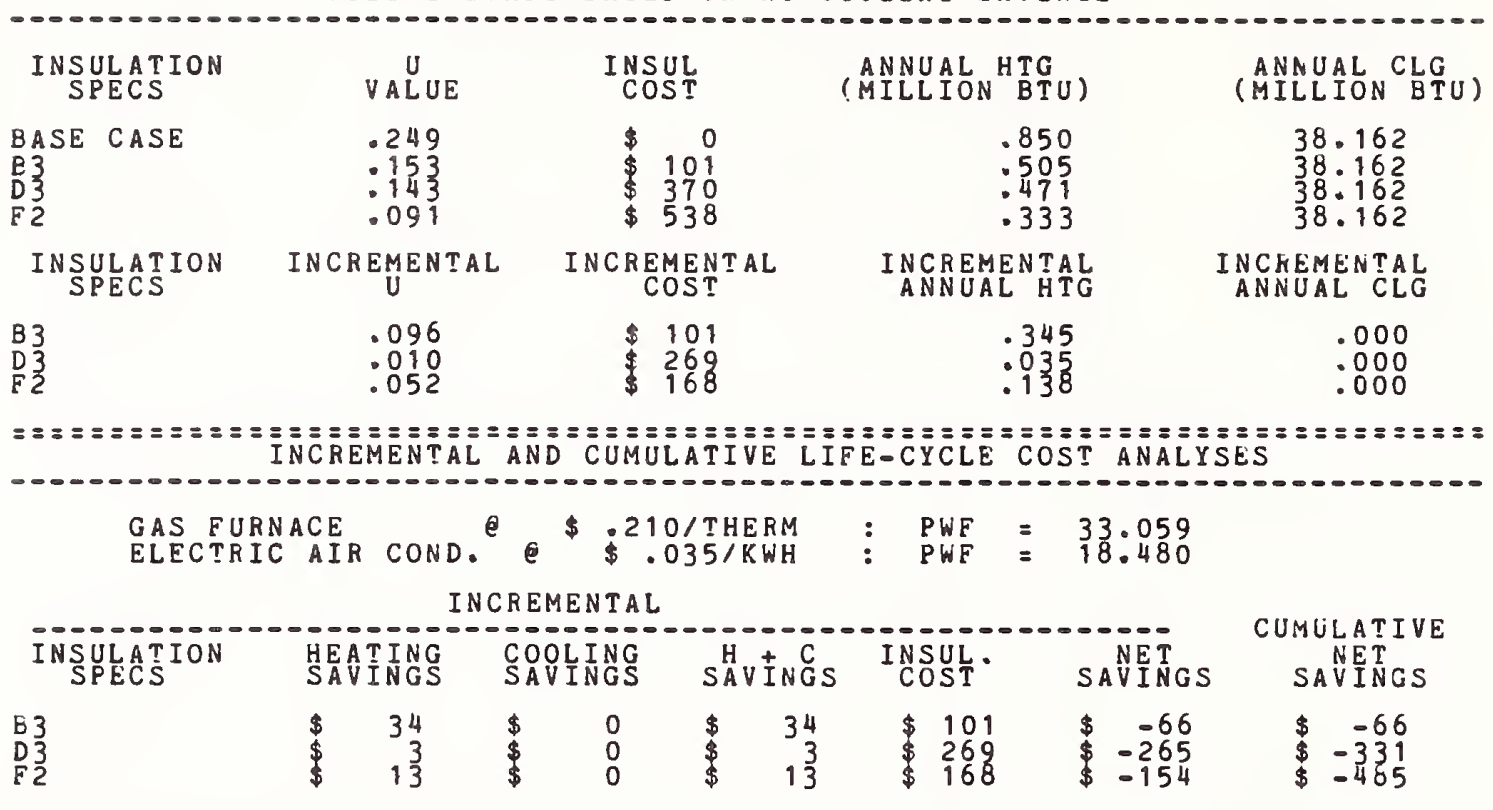

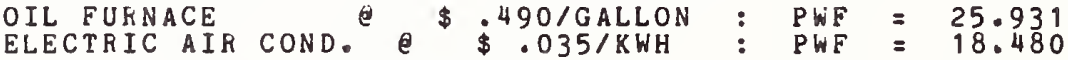

INCREMENTAL

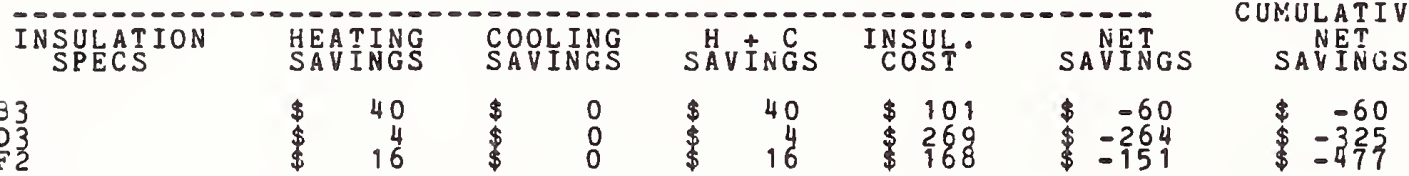

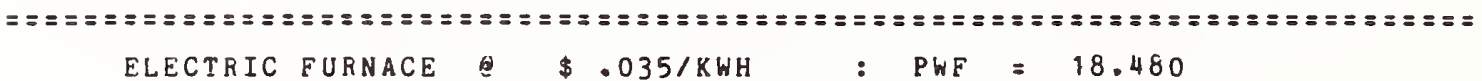
ELECTRIC AIR COND. $\$ .035 / \mathrm{KWH}: \mathrm{PWF}=18.480$

INCFEMENTAL

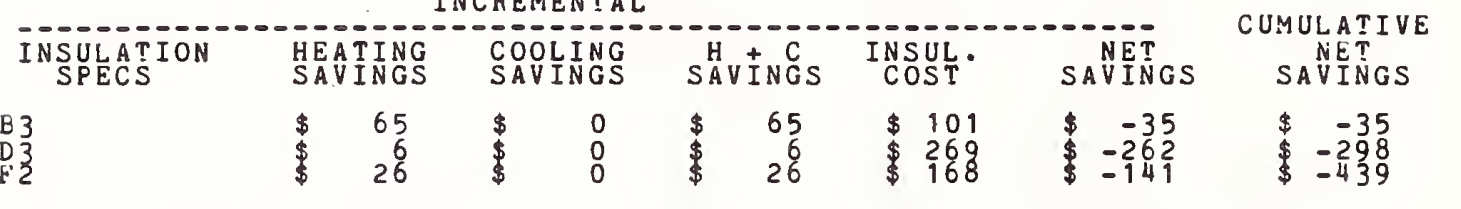

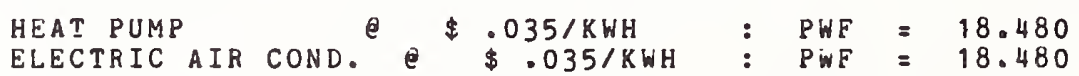

INCKEMENTAL

INSULATION HEATING SOPLING SHUTCS INSUT. SAVETG CUMULATIVE

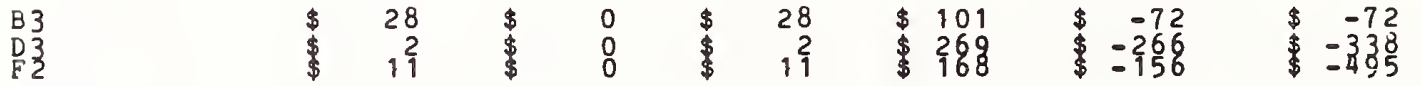


HUD2

WASHINGTON D.C ${ }_{1200}$ SQ FT HOUSE 8 IN BLOCK (OPEN CORE) COOLING LOADS BASED ON NO COOLING SAVINGS

\begin{tabular}{|c|c|c|c|c|}
\hline $\begin{array}{l}\text { INSULATION } \\
\text { SPECS }\end{array}$ & VALUE & $\begin{array}{l}\text { INSUL } \\
\text { COST }\end{array}$ & $\begin{array}{l}\text { ANNUAL }{ }^{\text {HTG }} \\
\text { (MILLION }\end{array}$ & $\begin{array}{l}\text { ANNUAL CLG } \\
\text { (MILLION BTU) }\end{array}$ \\
\hline $\begin{array}{l}\text { BASE CASE } \\
\text { B3 } \\
\text { D3 } \\
\text { F2 }\end{array}$ & $\begin{array}{r}.249 \\
: 153 \\
: 143 \\
.099\end{array}$ & $\begin{array}{l}\$ 0 \\
\$ 101 \\
\$ 378 \\
\$ 538\end{array}$ & $\begin{array}{l}22.025 \\
15: 508 \\
14: 833 \\
11.377\end{array}$ & $\begin{array}{l}15 \cdot 734 \\
15: 734 \\
15: 734 \\
15: 734\end{array}$ \\
\hline $\begin{array}{l}\text { INSULATION } \\
\text { SPECS }\end{array}$ & INCREMENTAL & $\begin{array}{c}\text { INCREMENTAL } \\
\text { COST }\end{array}$ & $\begin{array}{l}\text { INCREMENTAL } \\
\text { ANNUAL HTG }\end{array}$ & $\begin{array}{l}\text { INCREMENTAL } \\
\text { ANNUAL CLG }\end{array}$ \\
\hline $\begin{array}{l}\text { B } 3 \\
\text { D } 3 \\
\text { F } 2\end{array}$ & $\begin{array}{l}.096 \\
.010 \\
.052\end{array}$ & $\begin{array}{l}\$ 101 \\
\$ 269 \\
\$ 168\end{array}$ & $\begin{array}{r}6.517 \\
.675 \\
3.457\end{array}$ & $\begin{array}{l}.000 \\
.000 \\
.000\end{array}$ \\
\hline
\end{tabular}

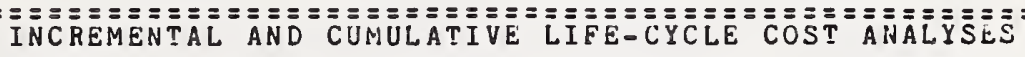

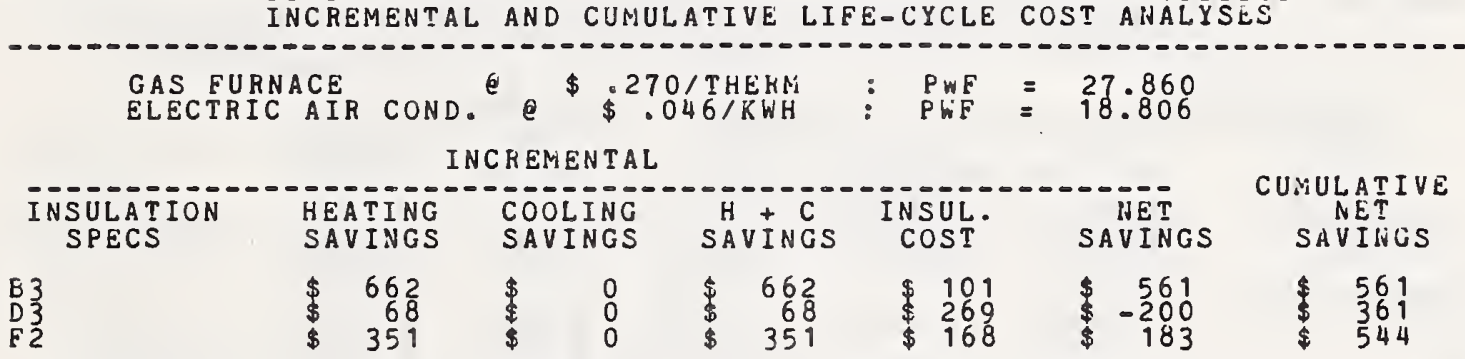

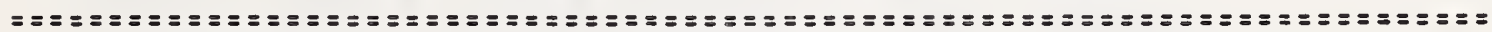

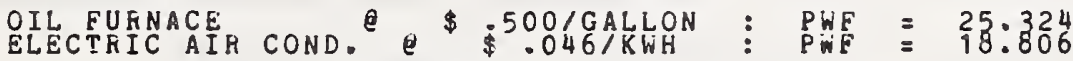

INCREMENTAL

\begin{tabular}{|c|c|c|c|c|c|c|c|c|c|c|c|}
\hline $\begin{array}{l}\text { INSULATION } \\
\text { SPECS }\end{array}$ & & $\begin{array}{l}\text { TTING } \\
\text { UINGS }\end{array}$ & & $\begin{array}{l}\bar{N} \bar{G} \\
G S\end{array}$ & & $\begin{array}{l}+\bar{C} \\
\text { I NGS }\end{array}$ & $\begin{array}{l}\text { INSUL. } \\
\text { COST }\end{array}$ & & $\begin{array}{l}\text { NET } \\
\text { VINGS }\end{array}$ & $\begin{array}{r}\mathrm{CU} \\
\mathrm{S}\end{array}$ & $\begin{array}{l}\text { LATIVE } \\
\text { ET } \\
\text { VINGS }\end{array}$ \\
\hline B 3 & $\begin{array}{l}\$ \\
\$\end{array}$ & $\begin{array}{r}755 \\
78 \\
400\end{array}$ & $\$$ & $\begin{array}{l}0 \\
0 \\
0\end{array}$ & $\$$ & $\begin{array}{l}755 \\
780\end{array}$ & $\begin{array}{l}101 \\
\$ 269 \\
\$ 168\end{array}$ & $\$$ & $\begin{array}{r}654 \\
-190 \\
232\end{array}$ & $\$$ & $\begin{array}{l}654 \\
463 \\
696\end{array}$ \\
\hline
\end{tabular}

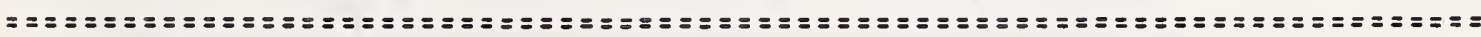

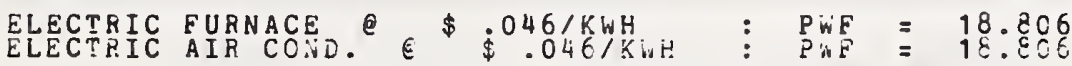

IAChENENTAL

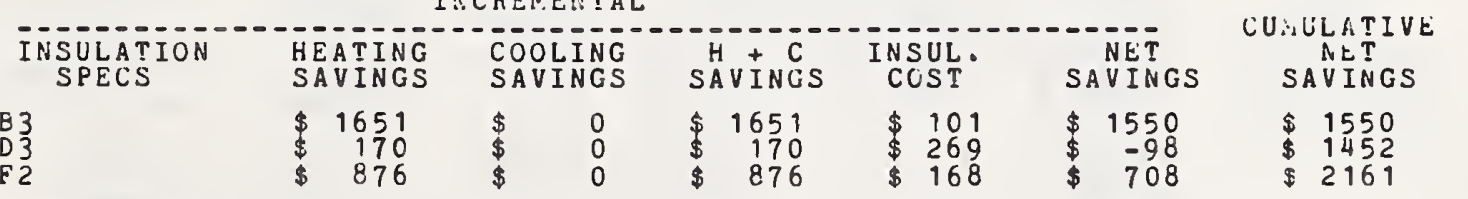

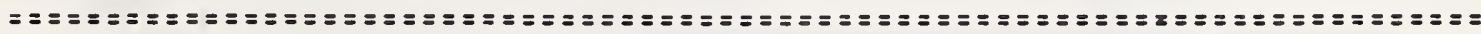
HEATPUMP

INCREMENTAL

\begin{tabular}{|c|c|c|c|c|c|c|}
\hline $\begin{array}{l}\text { INSULATION } \\
\text { SPECS }\end{array}$ & $\begin{array}{l}\text { HEAT ING } \\
\text { SAVINGS }\end{array}$ & $\begin{array}{l}\text { COOLING } \\
\text { SAVINGS }\end{array}$ & $\begin{array}{l}\mathrm{H}+\mathrm{C} \\
\text { SAVINGS }\end{array}$ & $\begin{array}{l}\text { INSUL. } \\
\text { COST }\end{array}$ & $\begin{array}{l}\text { NET } \\
\text { SAVINGS }\end{array}$ & $\begin{array}{l}\text { CUMULATIVE } \\
\text { SAVINGS }\end{array}$ \\
\hline & $\begin{array}{r}805 \\
\$ \quad 83 \\
\$ \quad 427\end{array}$ & $\$$ & $\begin{array}{r}805 \\
83 \\
427\end{array}$ & $\begin{array}{l}101 \\
\$ 269 \\
\$ 168\end{array}$ & $\begin{array}{r}704 \\
-185 \\
\$ \\
\$\end{array}$ & $\begin{array}{r}704 \\
\$ 519 \\
\$ 778\end{array}$ \\
\hline
\end{tabular}

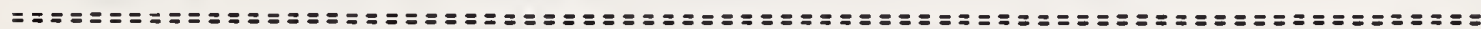


HUD 3

ALEUQUERQUE NEW MEXICO 1200 SQ FT HOUSE ${ }^{8}$ IN BLOCK (PERLITE FILLED) COOLING LOADS BASED ON NO COOLING SAVINGS

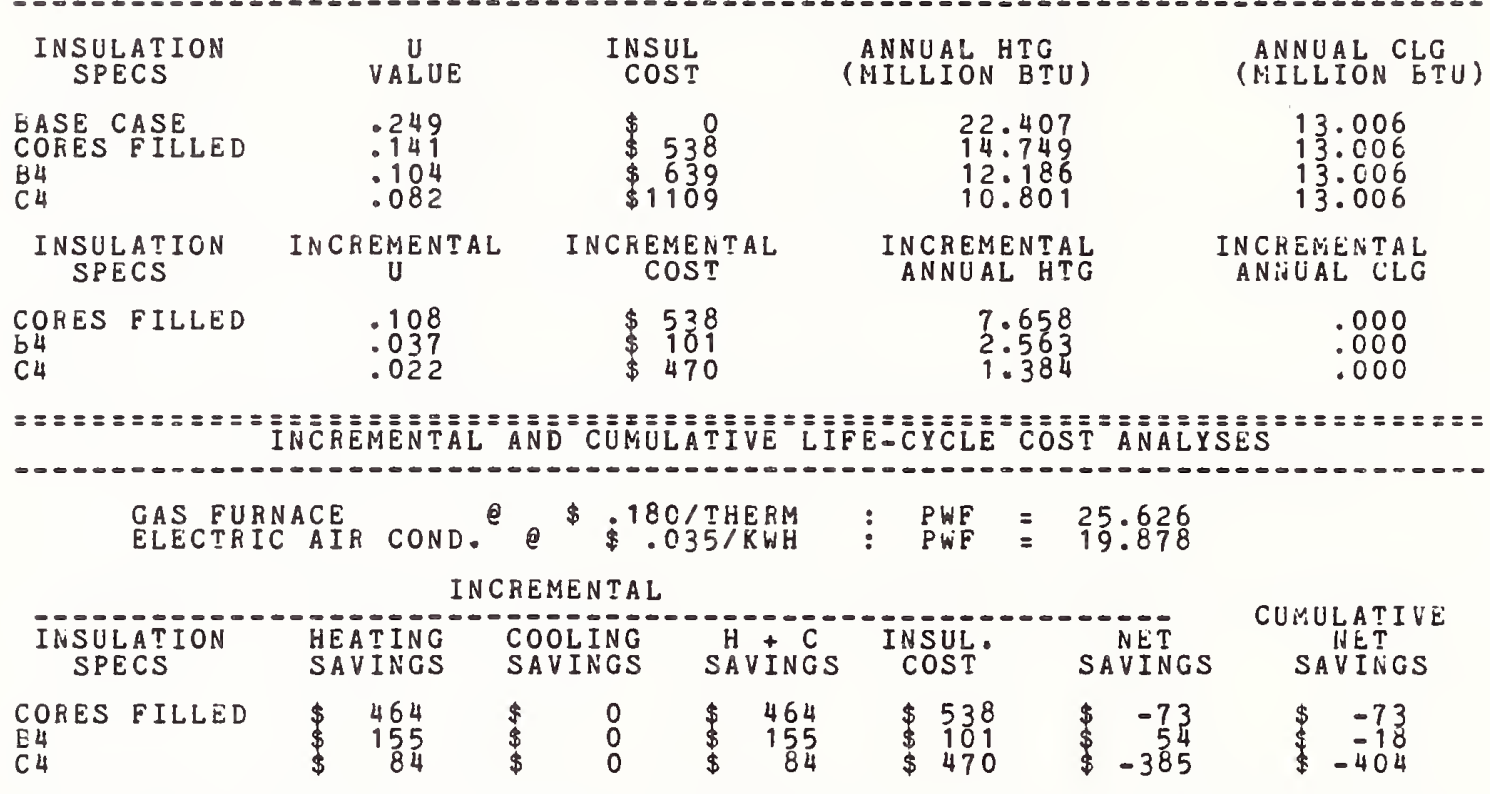

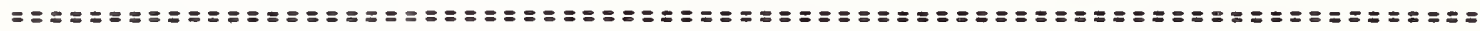

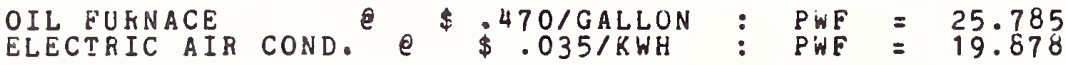
INCREMENTAL

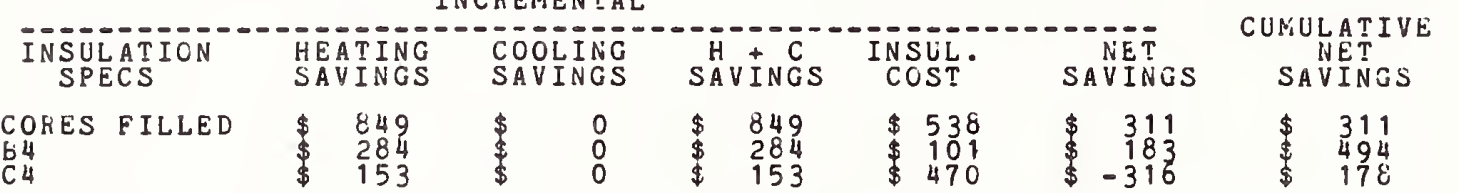

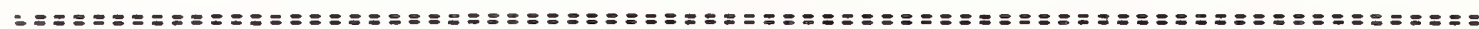

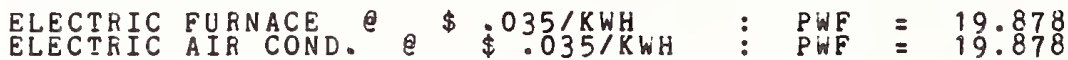

INCREMENTAL

\begin{tabular}{|c|c|c|c|c|c|c|}
\hline $\begin{array}{l}\text { INSULATION } \\
\text { SPECS }\end{array}$ & $\begin{array}{l}\text { HEATING } \\
\text { SAVINGS }\end{array}$ & $\begin{array}{l}\text { COOLING } \\
\text { SAVINGS }\end{array}$ & $\begin{array}{l}\mathrm{H}+\mathrm{C} \\
\mathrm{SA} V I N G S\end{array}$ & $\begin{array}{l}\text { INSUL: } \\
\text { COST }\end{array}$ & $\begin{array}{l}\text { NET } \\
\text { SAVINGS }\end{array}$ & $\begin{array}{c}\text { CUMULATIVE } \\
\text { NETT } \\
\text { SAVINGS }\end{array}$ \\
\hline $\begin{array}{l}\mathrm{CORES} \\
\mathrm{C} 4 \\
\mathrm{C} 4\end{array}$ & $\begin{array}{r}\$ 1561 \\
\$ 522 \\
\$ \quad 282\end{array}$ & $\$$ & $\begin{array}{r}\$ 1561 \\
\$ \quad 522 \\
\$ \quad 282\end{array}$ & $\begin{array}{l}\$ 538 \\
\$ 101 \\
\$ 470\end{array}$ & $\begin{array}{r}1023 \\
421 \\
\$-187\end{array}$ & $\begin{array}{l}\$ 1023 \\
\$ 1444 \\
\$ 1256\end{array}$ \\
\hline
\end{tabular}

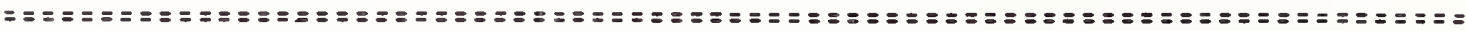
HEAT PUMP INCREMENTAL

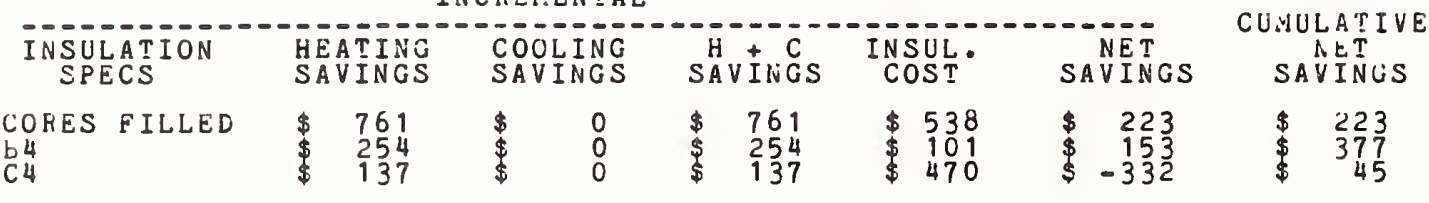


HUD3 ATLANTA GEORGIA 1200 SQ FT HOUSE 8 IN BLOCK (PERLITE FILLED) COOLING LOADS BASED ON NO COOLING SAVINGS

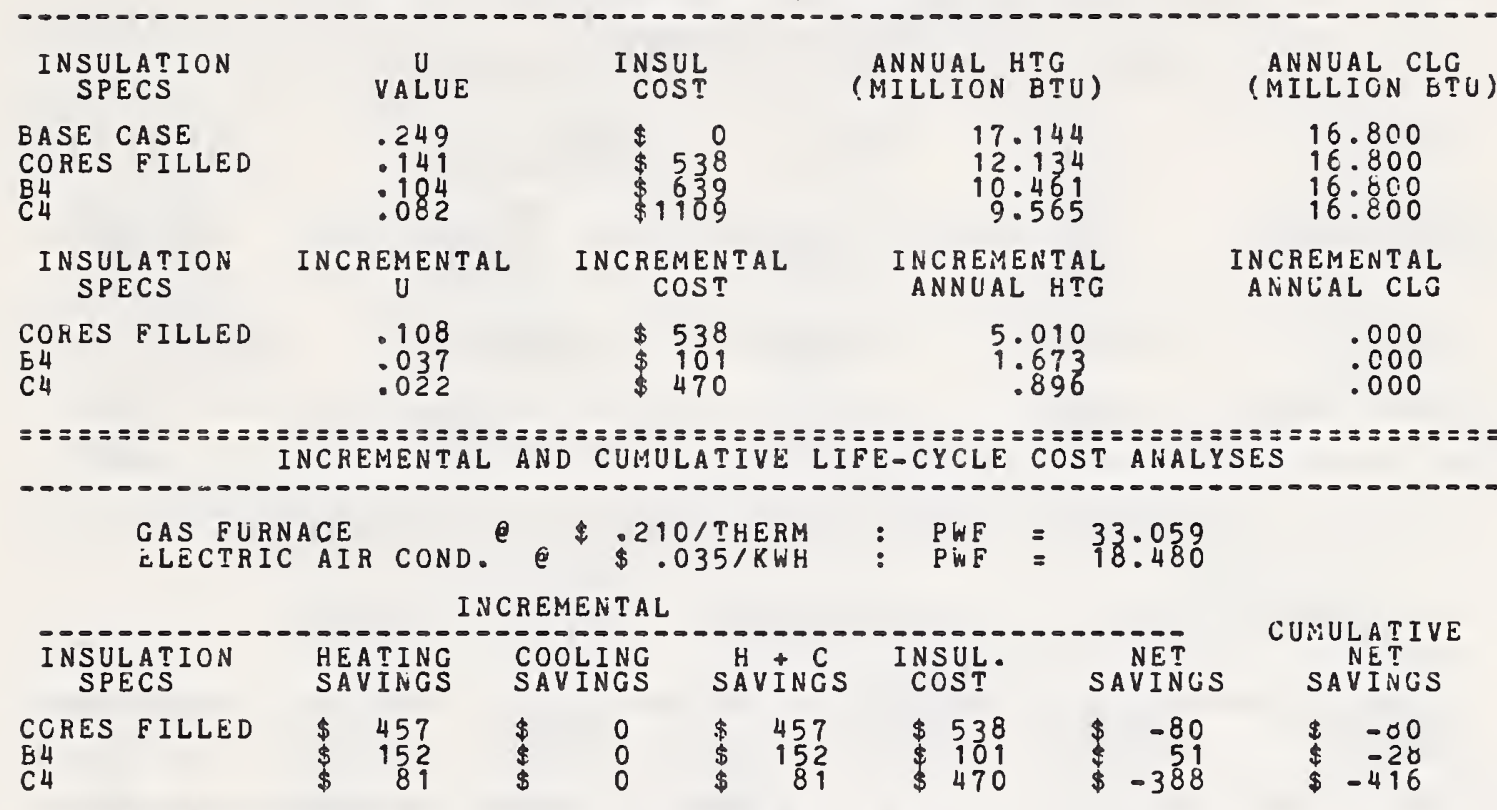

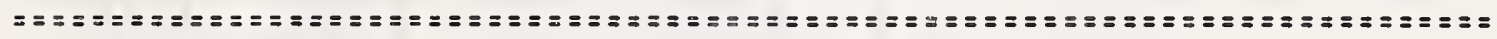

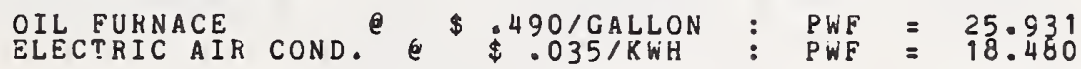
INCREMENTAL

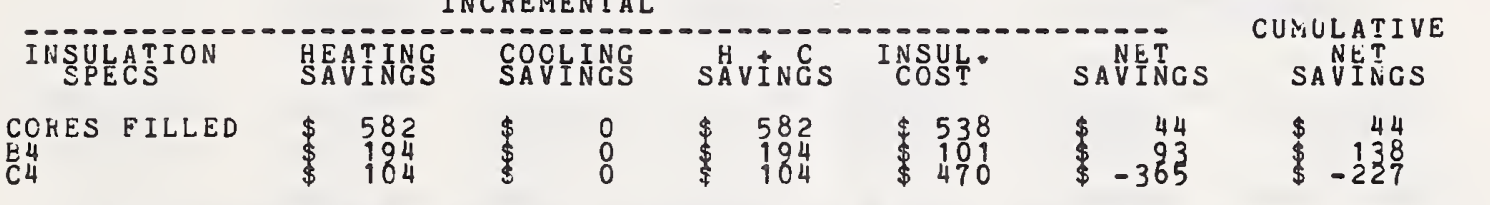

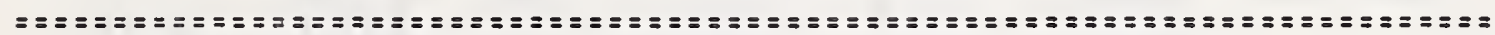
ELECTRIC FURNACE
ELECTRIC AIR COND. INCREMENTAL

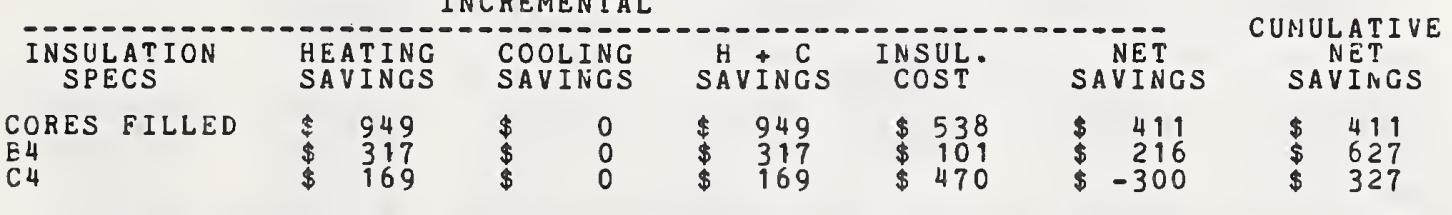

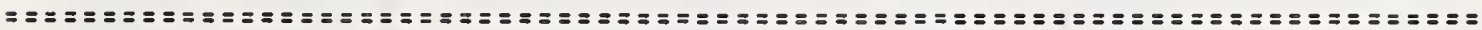

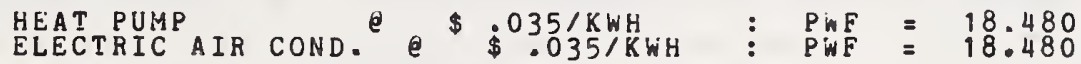

INCREMENTAL

\begin{tabular}{|c|c|c|c|c|c|c|}
\hline $\begin{array}{l}\text { INSULATION } \\
\text { SPECS }\end{array}$ & $\begin{array}{l}\text { HEATING } \\
\text { SAVINGS }\end{array}$ & $\begin{array}{l}\text { COOLING } \\
\text { SAVINGS }\end{array}$ & SÄVİ NGS & $\begin{array}{l}\text { INSUL. } \\
\text { COST. }\end{array}$ & SAVET INGS & $\begin{array}{l}\text { CUNULAT IVE } \\
\text { NETT } \\
\text { SAVINGS }\end{array}$ \\
\hline $\begin{array}{l}\text { CORES FILLED } \\
\text { C } 4 \\
C 4\end{array}$ & $\begin{array}{l}\quad 441 \\
147 \\
\$ \quad 78\end{array}$ & $\$$ & $\begin{array}{r}\$ 41 \\
\$ \quad 147 \\
\$ \quad 78\end{array}$ & $\begin{array}{l}538 \\
\$ 101 \\
\$ 470\end{array}$ & $\begin{array}{r}-96 \\
46 \\
-391\end{array}$ & $\begin{array}{r}-96 \\
\$-49 \\
\$-440\end{array}$ \\
\hline
\end{tabular}

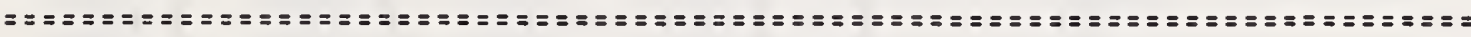


HUD 3

INDIANAPOLIS INDIANA COOLING LOADS BASEDON NO COOLING SAVINGS

8 IN BLOCK (PERLITE FILLED)

\begin{tabular}{|c|c|c|c|c|}
\hline $\begin{array}{l}\text { INSULATION } \\
\text { SPECS }\end{array}$ & $\stackrel{U}{\text { VALUE }}$ & $\begin{array}{l}\text { INSUL } \\
\text { COST }\end{array}$ & $\begin{array}{l}\text { ANNUAL HTG } \\
\text { (MILLION BTU) }\end{array}$ & $\begin{array}{l}\text { ANNUAL CLG } \\
\text { (MILLON BTU) }\end{array}$ \\
\hline $\begin{array}{l}\text { EASE CASE } \\
\text { CORES FILLED } \\
\text { E4 } \\
\text { C } 4\end{array}$ & $\begin{array}{r}.249 \\
.141 \\
.104 \\
.082\end{array}$ & $\begin{array}{r}\$ \\
538 \\
\$ 39 \\
\$ 1109\end{array}$ & $\begin{array}{l}42.110 \\
30.748 \\
25.851 \\
24.522\end{array}$ & $\begin{array}{l}10.652 \\
10.652 \\
10.652 \\
10.652\end{array}$ \\
\hline $\begin{array}{l}\text { INSULAT ION } \\
\text { SPECS }\end{array}$ & INCREMENTAL & $\begin{array}{l}\text { INCREMENTAL } \\
\text { COST }\end{array}$ & $\begin{array}{l}\text { INCREMENTAL } \\
\text { ANNUAL HTG }\end{array}$ & $\begin{array}{l}\text { INCREMENTAL } \\
\text { ANNUAL CLG }\end{array}$ \\
\hline $\begin{array}{l}\text { CORES FILLED } \\
\text { B } 4 \\
\text { C } 4\end{array}$ & $\begin{array}{l}.108 \\
.037 \\
.022\end{array}$ & $\begin{array}{l}538 \\
\$ 101 \\
\$ 470\end{array}$ & $\begin{array}{r}11.362 \\
3: 897 \\
2.329\end{array}$ & $\begin{array}{l}.000 \\
.000 \\
.000\end{array}$ \\
\hline
\end{tabular}

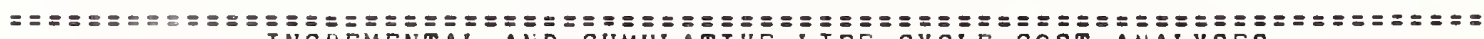
INCREMENTAL AND CUMULATIVE LIFE-CYCLE COST ANALYSES

INCREMENTAL

\begin{tabular}{|c|c|c|c|c|c|c|c|c|}
\hline $\begin{array}{l}\text { INSULAT ION } \\
\text { SPECS }\end{array}$ & $\begin{array}{l}\text { HEATING } \\
\text { SAVINGS }\end{array}$ & & $\begin{array}{l}\text { NG } \\
\text { GS }\end{array}$ & SÄVİNGS & $\begin{array}{l}\text { INSUL } \\
\text { COST }\end{array}$ & $\begin{array}{l}\text { NET } \\
\text { SAVINGS }\end{array}$ & $\mathrm{SA}$ & $\begin{array}{l}\text { LAT I VE } \\
\text { NET } \\
\text { VINGS }\end{array}$ \\
\hline $\begin{array}{l}\text { CORES FILLED } \\
\text { B } 4 \\
\text { C } 4\end{array}$ & $\begin{array}{r}\$ 1165 \\
\$ 390 \\
\$ 338\end{array}$ & $\$$ & $\begin{array}{l}0 \\
0 \\
0\end{array}$ & $\begin{array}{r}1165 \\
\$ 399 \\
\$ \quad 238\end{array}$ & $\begin{array}{l}538 \\
\$ 101 \\
\$ 470\end{array}$ & $\begin{array}{r}627 \\
\$ 298 \\
\$-231\end{array}$ & $\$$ & $\begin{array}{l}627 \\
926 \\
695\end{array}$ \\
\hline
\end{tabular}

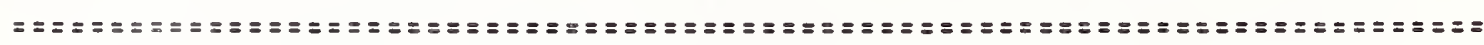

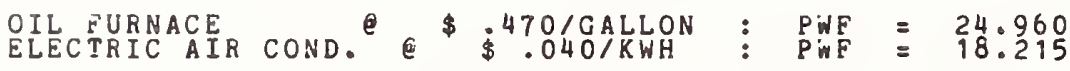

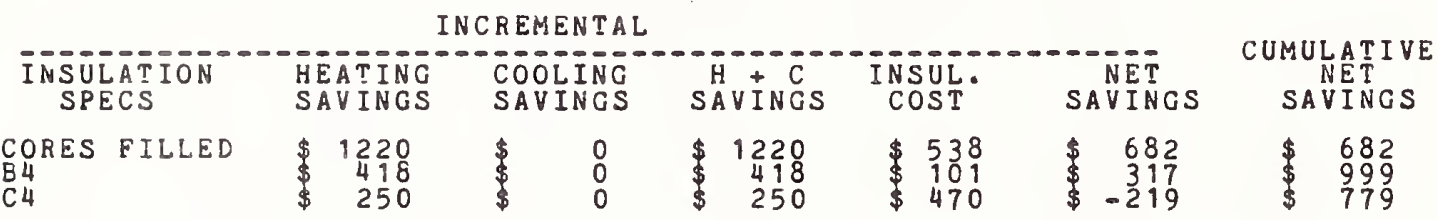

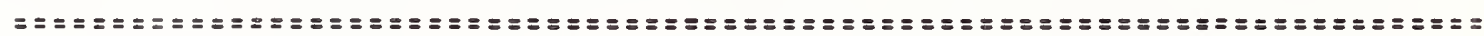
ELECTRIC FUKNACE
ELECTRIC AIR COND. $\$$ \$ $\$ 040 / \mathrm{KWH}$

INCREMENTAL

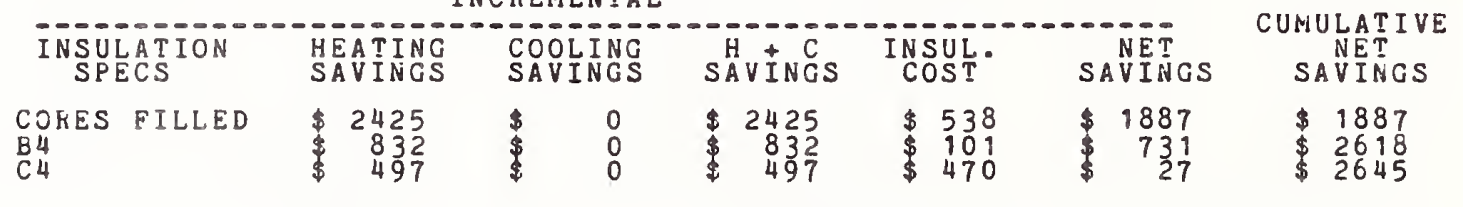

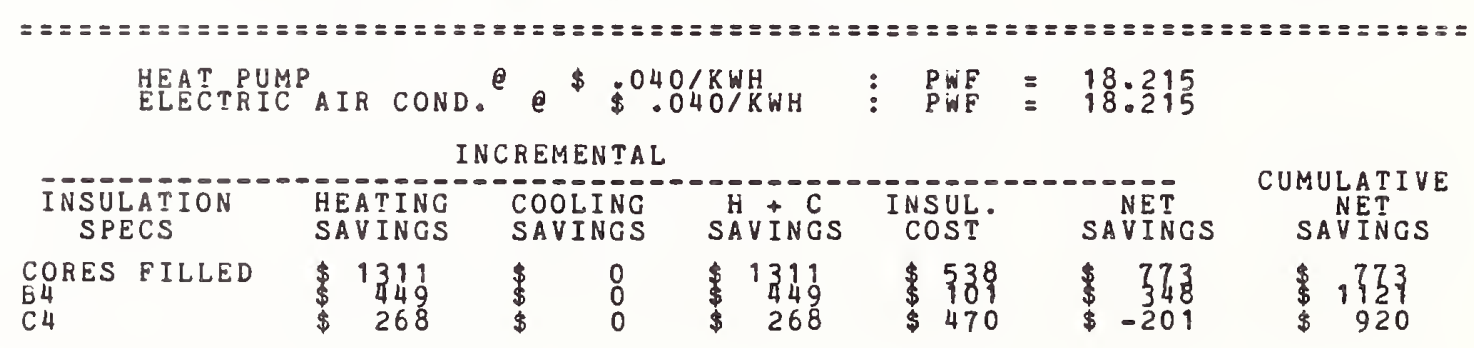

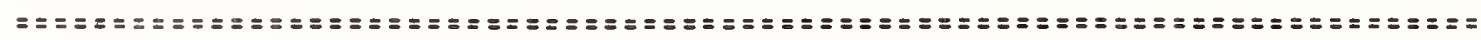


HUD 3 COOLING LOADS BASED ON NO COOLING SAVINGS

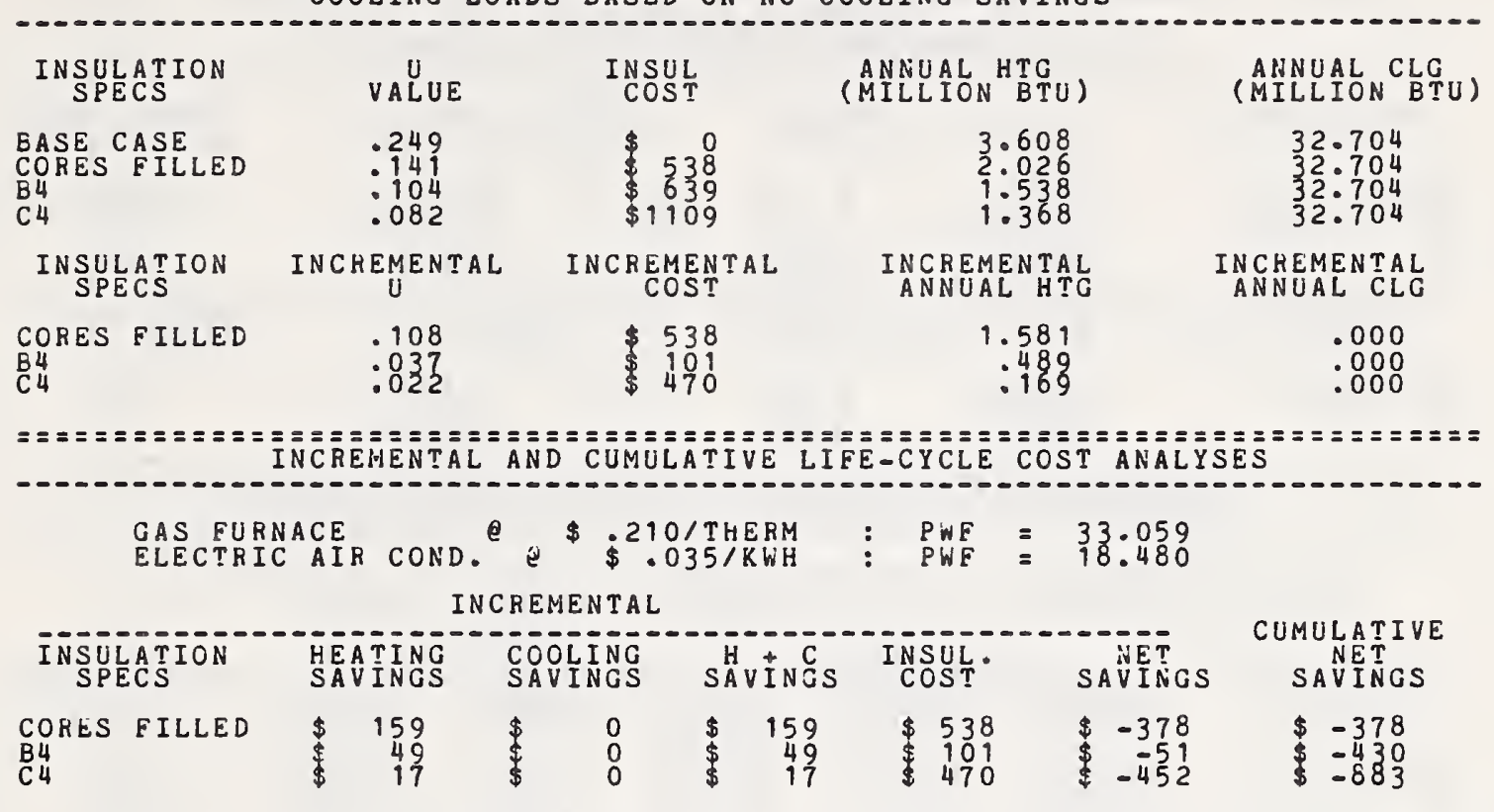

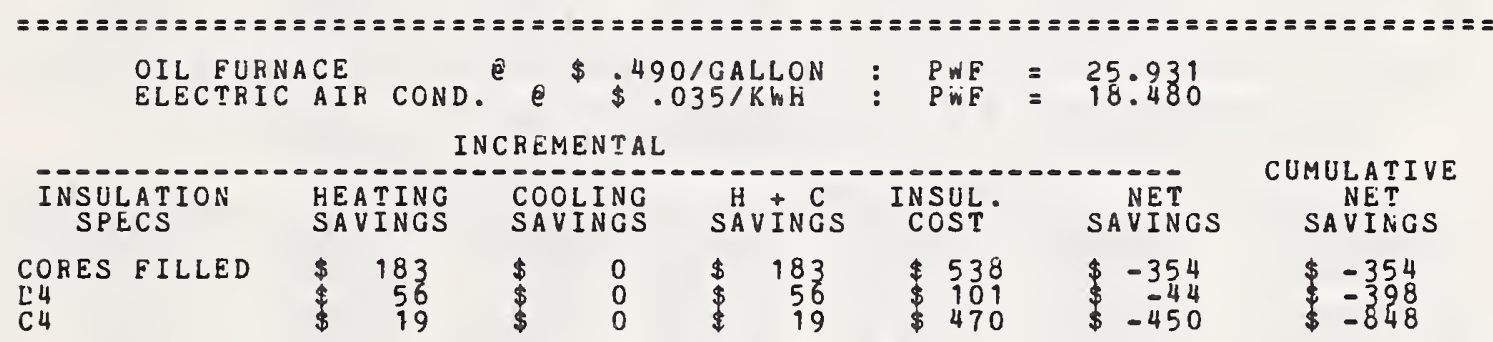

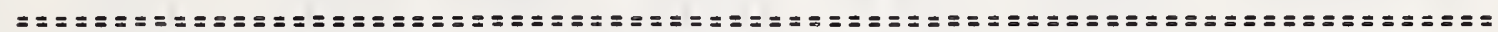

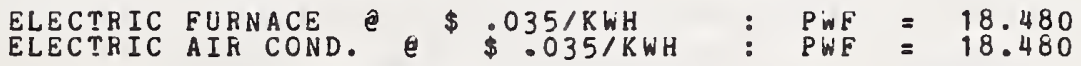

INCREMENTAL

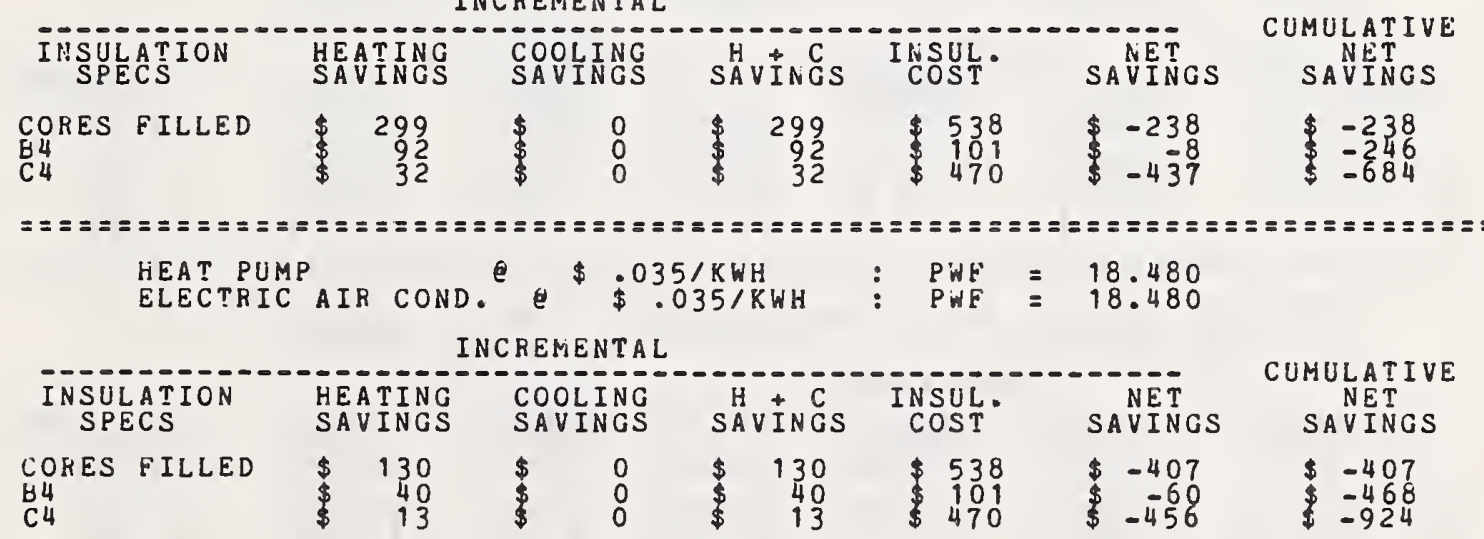


HUD 3

MADISON WISCONSIN ${ }_{1200}$ SQ FT HOUSE 8 IN BLOCK (PERLITE FILLED) COOLING LOADS BASED ON NO COOLING SAVINGS

\begin{tabular}{|c|c|c|c|c|}
\hline $\begin{array}{l}\text { INSULATION } \\
\text { SPECS }\end{array}$ & $\stackrel{U}{\text { VALUE }}$ & $\begin{array}{l}\text { INSUL } \\
\text { COST }\end{array}$ & $\begin{array}{l}\text { ANNUAL HTG } \\
\text { (MILLION BTU) }\end{array}$ & $\begin{array}{l}\text { ANNUAL CLG } \\
\text { (MILLION BTU) }\end{array}$ \\
\hline $\begin{array}{l}\text { BASE CASE } \\
\text { CORES FILLED } \\
\text { B } 4 \\
\text { C } 4\end{array}$ & $\begin{array}{r}.249 \\
: 149 \\
.104 \\
.082\end{array}$ & $\begin{array}{rr}\$ & 538 \\
\$ & 639 \\
\$ 1109\end{array}$ & $\begin{array}{l}49.664 \\
35: 556 \\
30: 725 \\
27.859\end{array}$ & $\begin{array}{l}5: 363 \\
5: 363 \\
5: 363 \\
5: 363\end{array}$ \\
\hline $\begin{array}{l}\text { INSULATION } \\
\text { SPECS }\end{array}$ & INCREMENTAL & $\begin{array}{c}\text { INCREMENTAL } \\
\text { COST }\end{array}$ & $\begin{array}{l}\text { INCREMENTAL } \\
\text { ANNUAL HTG }\end{array}$ & $\begin{array}{l}\text { INCREMENTAL } \\
\text { ANNUAL CLC }\end{array}$ \\
\hline $\begin{array}{l}\text { CORES FILLED } \\
\text { E4 } \\
\text { C4 }\end{array}$ & $\begin{array}{l}.108 \\
.037 \\
.022\end{array}$ & $\begin{array}{l}538 \\
\$ 909 \\
\$ 470\end{array}$ & $\begin{array}{r}14.108 \\
4.831 \\
2.866\end{array}$ & $\begin{array}{l}.000 \\
.000 \\
.000\end{array}$ \\
\hline
\end{tabular}

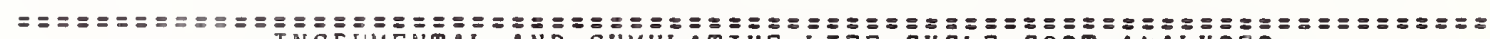
INCFEMENTALAND CUMULATIVE LIFE-CYCLE COST ANALYSES

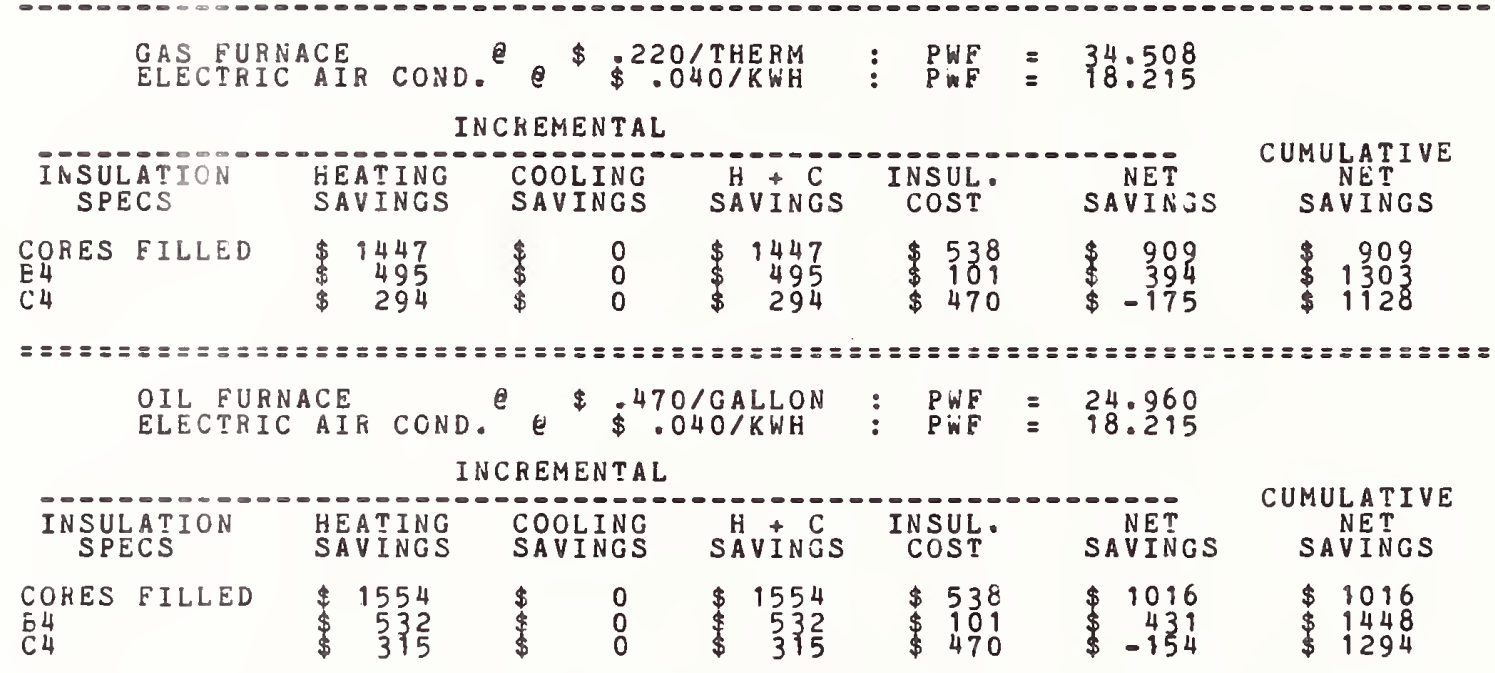

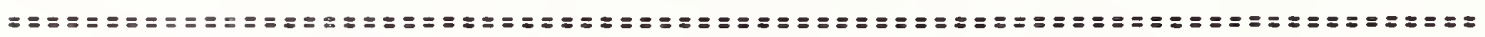

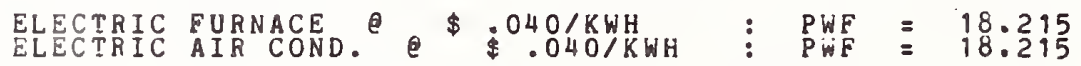

INCREMENTAL

\begin{tabular}{|c|c|c|c|c|c|c|c|}
\hline $\begin{array}{l}\text { INSULATION } \\
\text { SPECS }\end{array}$ & $\begin{array}{l}\text { HEATING } \\
\text { SAVINGS }\end{array}$ & & $\begin{array}{l}\text { ING } \\
N G S\end{array}$ & $\begin{array}{l}H+C^{\prime} \\
\text { SAVINGS }\end{array}$ & $\begin{array}{l}\text { INSUL. } \\
\text { COST }\end{array}$ & $\begin{array}{l}\text { NET } \\
\text { SAVINGS }\end{array}$ & $\begin{array}{c}\text { CUMULATIVE } \\
\text { NET T } \\
\text { SAVINGS }\end{array}$ \\
\hline${ }_{4}^{\text {CRES FILLED }}$ & 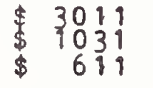 & $\$$ & $\begin{array}{l}0 \\
0 \\
0\end{array}$ & $\begin{array}{rl} & 3091 \\
10 & 31 \\
\$ & 611\end{array}$ & $\begin{array}{l}538 \\
\$ 101 \\
\$ 470\end{array}$ & $\begin{array}{r}2473 \\
\$ 930 \\
\$ \quad 941\end{array}$ & $\begin{array}{l}2473 \\
\$ 3404 \\
\$ 3545\end{array}$ \\
\hline
\end{tabular}

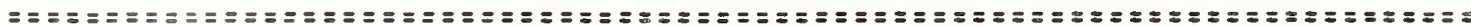

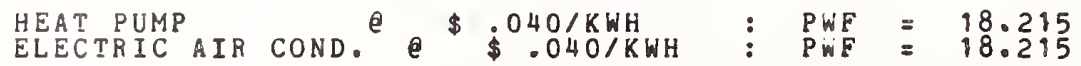

I NCREMENT AL

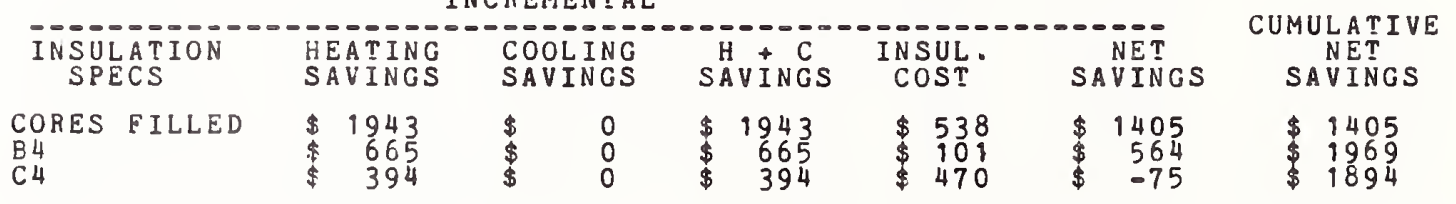

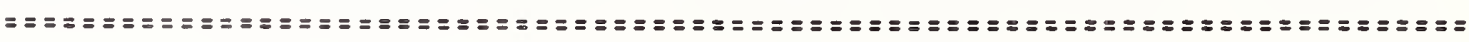


HUD 3

PHOENIX ARIZONA 1200 SO FT HOUSE 8 IN BLOCK (PERLITE FILLED) COOLING LOADS BASED ON NO COOLING SAVINGS

\begin{tabular}{|c|c|c|c|c|}
\hline $\begin{array}{l}\text { INSULATION } \\
\text { SPECS }\end{array}$ & $\stackrel{U}{\text { VALUE }}$ & $\begin{array}{l}\text { INSUL } \\
\text { COST }\end{array}$ & $\begin{array}{l}\text { ANNUAL HTG } \\
\text { (MILLION BTU) }\end{array}$ & $\begin{array}{l}\text { ANNUAL CLG } \\
\text { (MILLION BTU) }\end{array}$ \\
\hline $\begin{array}{l}\text { BASE CASE } \\
\text { COHES FILLED } \\
\text { B } 4 \\
\text { C } 4\end{array}$ & $\begin{array}{l}.249 \\
.141 \\
.104 \\
.082\end{array}$ & $\begin{array}{r}\$ 0 \\
538 \\
\$ 239 \\
\$ 1109\end{array}$ & $\begin{array}{l}4.055 \\
2.233 \\
1.680 \\
1.514\end{array}$ & $\begin{array}{l}42.003 \\
42.003 \\
42.003 \\
42.003\end{array}$ \\
\hline $\begin{array}{l}\text { INSULATION } \\
\text { SPECS }\end{array}$ & INCREMENTAL & $\begin{array}{c}\text { INCREMENTAL } \\
\text { COST }\end{array}$ & $\begin{array}{l}\text { INCREMENTAL } \\
\text { ANNUAL HTG }\end{array}$ & $\begin{array}{l}\text { INCFEMELTAL } \\
\text { ANNUAL CLG }\end{array}$ \\
\hline $\begin{array}{l}\text { CORES FILLED } \\
\text { E } 4 \\
\text { C } 4\end{array}$ & $\begin{array}{l}.108 \\
.037 \\
.022\end{array}$ & $\begin{array}{l}\$ 538 \\
\$ 101 \\
\$ 470\end{array}$ & $\begin{array}{r}1.822 \\
.553 \\
.166\end{array}$ & $\begin{array}{l}.000 \\
.000 \\
.000\end{array}$ \\
\hline
\end{tabular}

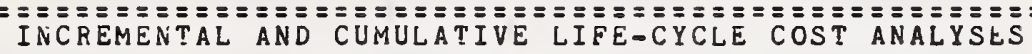

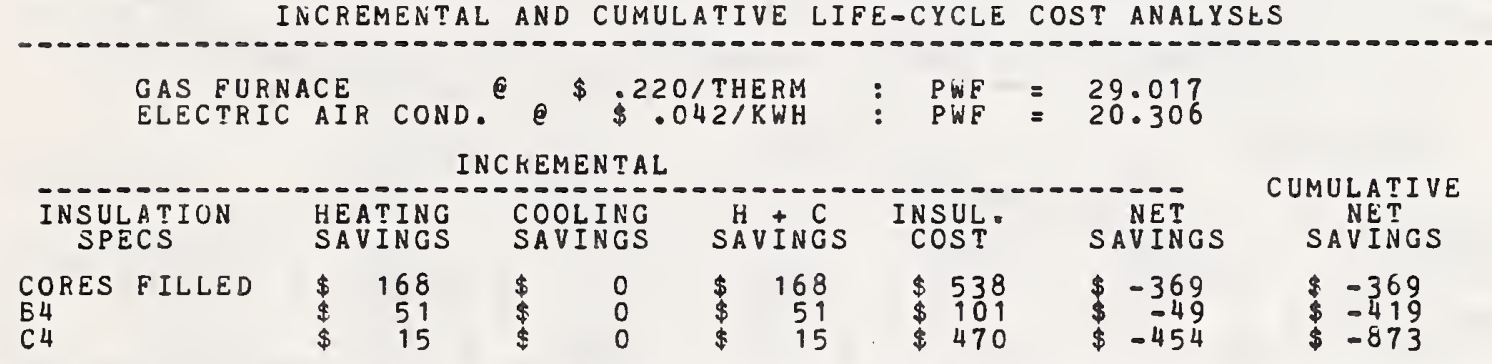

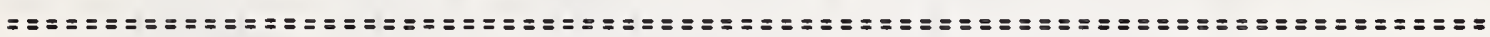

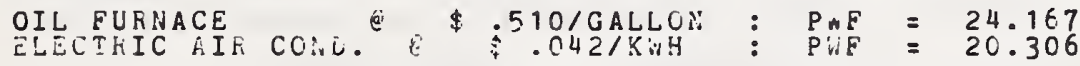

I:CFEIIENTAL

\begin{tabular}{|c|c|c|c|c|c|c|}
\hline INSULATION & $\begin{array}{l}\text { HEATING } \\
\text { SAVINGS }\end{array}$ & $\begin{array}{l}\text { COOLING } \\
\text { SAVINGS }\end{array}$ & SÄV I NGS & $\begin{array}{l}\text { INSUL. } \\
\text { COST }\end{array}$ & SAVIETES & $\begin{array}{c}\text { CUNIULATIVE } \\
\text { NET } \\
\text { SAVINGS }\end{array}$ \\
\hline $\begin{array}{l}\text { COHES FILLED } \\
044\end{array}$ & $\begin{array}{r}205 \\
\quad 62 \\
\$ \quad 18\end{array}$ & $\$$ & $\begin{array}{r}205 \\
62 \\
18\end{array}$ & $\begin{array}{l}538 \\
\$ 101 \\
\$ 470\end{array}$ & $\begin{array}{l}-332 \\
\$-451\end{array}$ & $\begin{array}{l}-332 \\
\$-370 \\
\$-822\end{array}$ \\
\hline
\end{tabular}

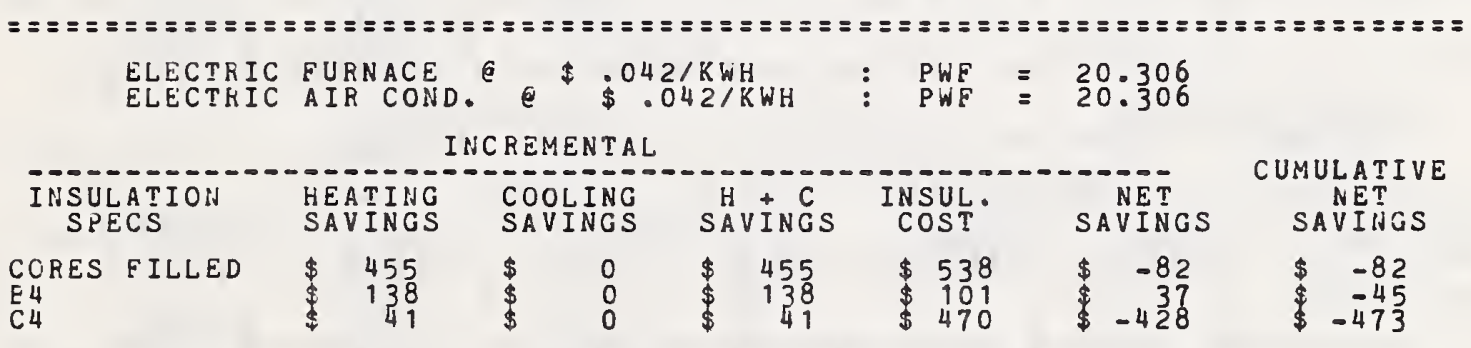

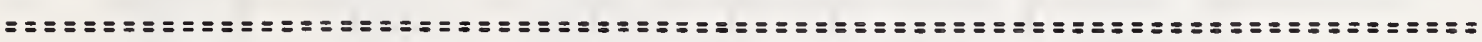

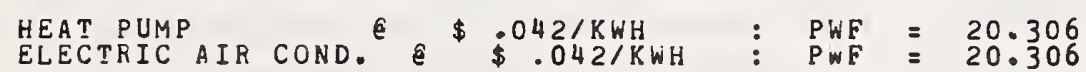
INCREMENTAL

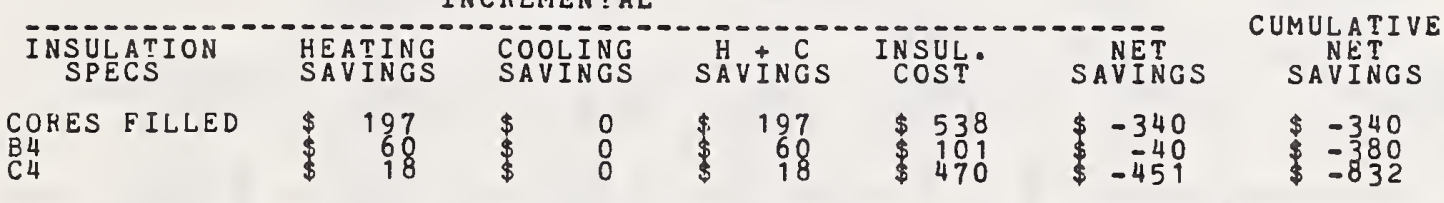


HUD 3

TAMPA FLORIDA 1200 SQ FT HOUSE 8 IN ELOCK (PEKLITE FILLED) COOLING LOADS BASED ON NO COOLING SAVINGS

\begin{tabular}{|c|c|c|c|c|}
\hline $\begin{array}{l}\text { INSULATION } \\
\text { SPECS }\end{array}$ & VALUE & $\begin{array}{l}\text { INSUL } \\
\text { COST }\end{array}$ & $\begin{array}{l}\text { ANNUAL HTG } \\
\text { (MILLIOH BTU) }\end{array}$ & $\begin{array}{l}\text { ANNUAL CLC } \\
\text { (HILLION BTU) }\end{array}$ \\
\hline $\begin{array}{l}\text { CASE CASE } \\
\text { CORES FILLED } \\
\text { B4 } \\
\text { C4 }\end{array}$ & $\begin{array}{l}.249 \\
.141 \\
.104 \\
.082\end{array}$ & $\begin{array}{rr}\$ & 0 \\
\$ & 538 \\
539 \\
\$ 1109\end{array}$ & $\begin{array}{r}.854 \\
.415 \\
.281 \\
.239\end{array}$ & $\begin{array}{l}38 \cdot 162 \\
38: 162 \\
38: 162 \\
38: 162\end{array}$ \\
\hline $\begin{array}{l}\text { INSULATION } \\
\text { SPECS }\end{array}$ & INCEE & $\begin{array}{c}\text { INCKEMENTAL } \\
\text { COST }\end{array}$ & $\begin{array}{l}\text { INCREPENTAL } \\
\text { ANNUAL HTG }\end{array}$ & $\begin{array}{l}\text { INCKEMENTAL } \\
\text { ANIVLAL CLG }\end{array}$ \\
\hline $\begin{array}{l}\text { CCRES FILLED } \\
\text { B } 4 \\
C 4\end{array}$ & $\begin{array}{l}.108 \\
.037 \\
.022\end{array}$ & $\begin{array}{l}\$ 538 \\
\$ 101 \\
\$ 470\end{array}$ & $\begin{array}{l}.439 \\
.134 \\
.042\end{array}$ & $\begin{array}{l}.000 \\
.000 \\
.000\end{array}$ \\
\hline
\end{tabular}

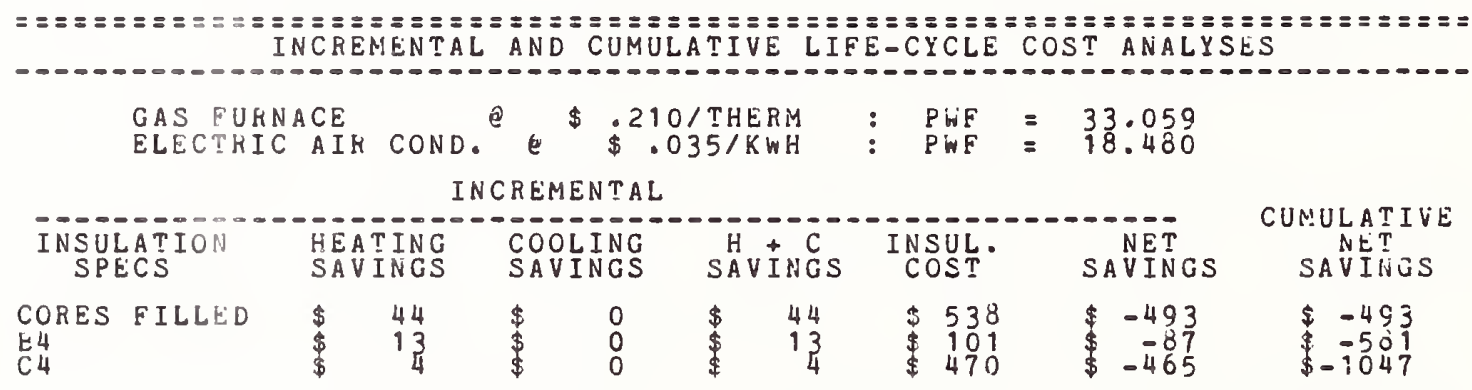

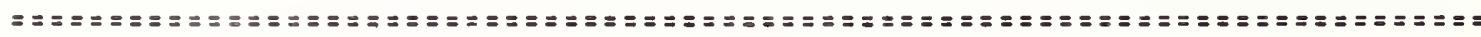

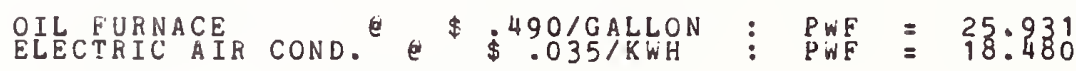

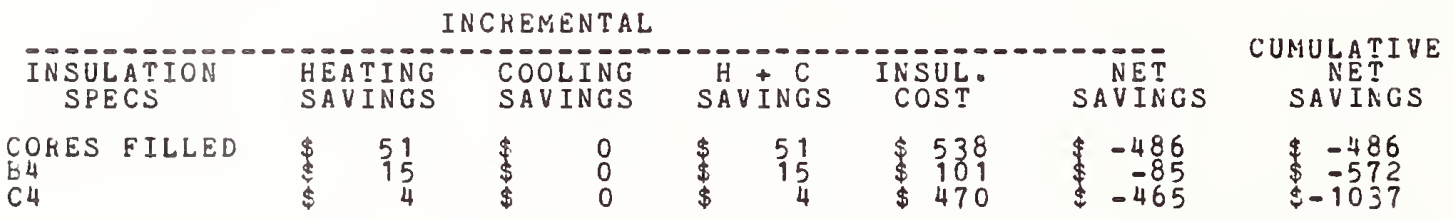

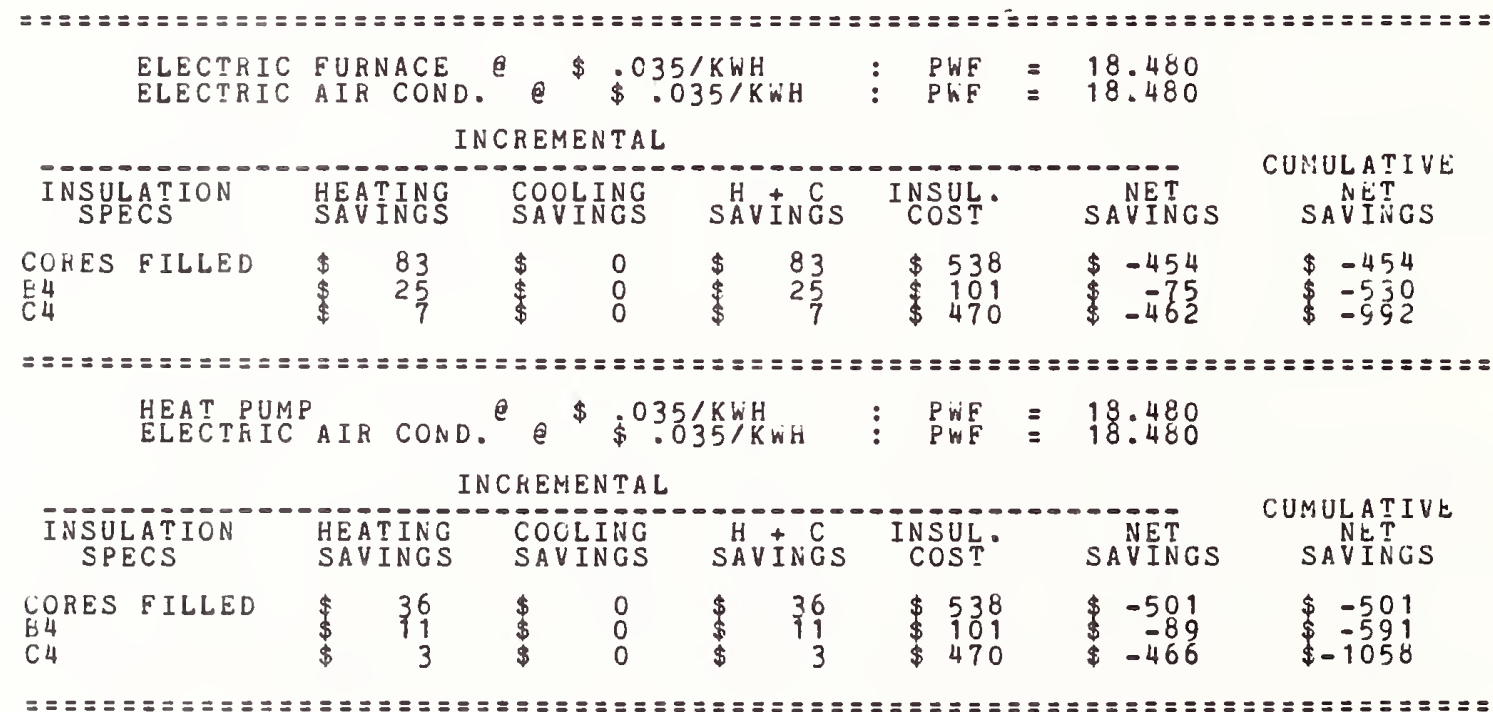


HUD 3

WASHINGTON D.C.

8 IN BLOCK (PERLITE FILLED) COOLING LOADS BRSEDSONFOHQUSEING SAVINGS

\begin{tabular}{|c|c|c|c|c|}
\hline $\begin{array}{l}\text { INSULATION } \\
\text { SPECS }\end{array}$ & VALUE & $\begin{array}{l}\text { INSUL } \\
\text { COST }\end{array}$ & $\begin{array}{l}\text { ANNUAL }{ }^{H T G} \\
\text { (MILLION }\end{array}$ & $\begin{array}{l}\text { ANNUAL CLG } \\
\text { (MILLION BTU) }\end{array}$ \\
\hline $\begin{array}{l}\text { BASE CASE } \\
\text { CORES FILLED } \\
\text { E4 } \\
\text { C } 4\end{array}$ & $\begin{array}{r}.249 \\
: 141 \\
104 \\
: 082\end{array}$ & $\begin{array}{r}\$ 0 \\
\$ 38 \\
\$ 339 \\
\$ 1109\end{array}$ & $\begin{array}{l}22.035 \\
14: 589 \\
12.058 \\
10.603\end{array}$ & $\begin{array}{l}15.674 \\
15.674 \\
15.674 \\
15.674\end{array}$ \\
\hline INSULATION & I NCREMENTAL & $\begin{array}{c}\text { INCREMENTAL } \\
\text { COST }\end{array}$ & $\begin{array}{l}\text { INCREMENTAL } \\
\text { ANNUAL HTG }\end{array}$ & $\begin{array}{l}\text { INCREMENTAL } \\
\text { ANNUAL CLG }\end{array}$ \\
\hline $\begin{array}{l}\text { CORES FILLED } \\
\text { B } 4 \\
C 4\end{array}$ & $\begin{array}{r}.108 \\
.037 \\
.022\end{array}$ & $\begin{array}{l}538 \\
101 \\
\$ 470\end{array}$ & $\begin{array}{l}7: 446 \\
2: 531 \\
1.455\end{array}$ & $\begin{array}{l}.000 \\
.000 \\
.000\end{array}$ \\
\hline
\end{tabular}

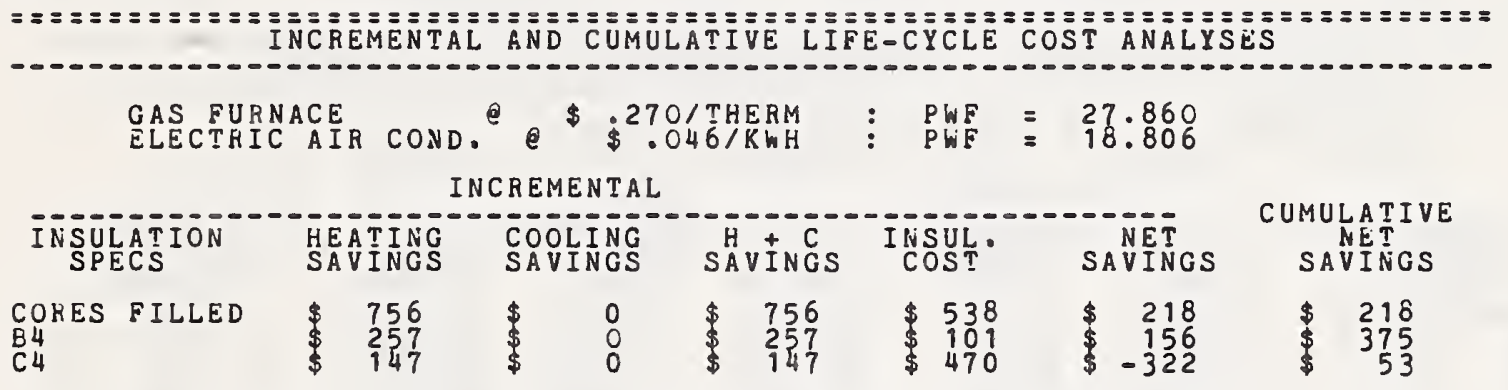
$\begin{array}{lll}\text { OIL FURNACE } & 6 \text { \$5O0/GALLON } \\ \text { ELECTRIC AIR COND. } & \$ \text { PWF } & =25.324 \\ \text { PWF } & =18.046 / K W H\end{array}$

INCREMENTAL

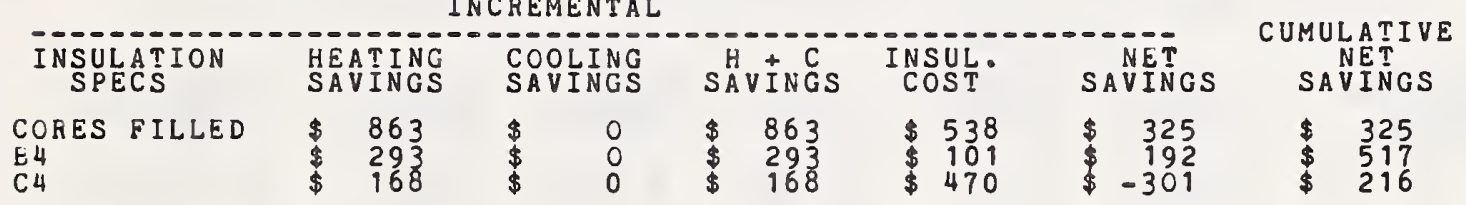

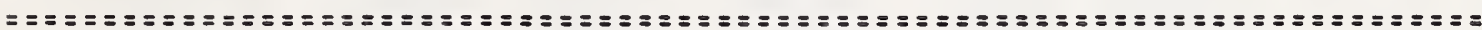

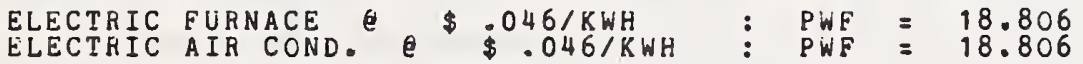

INCREMENTAL

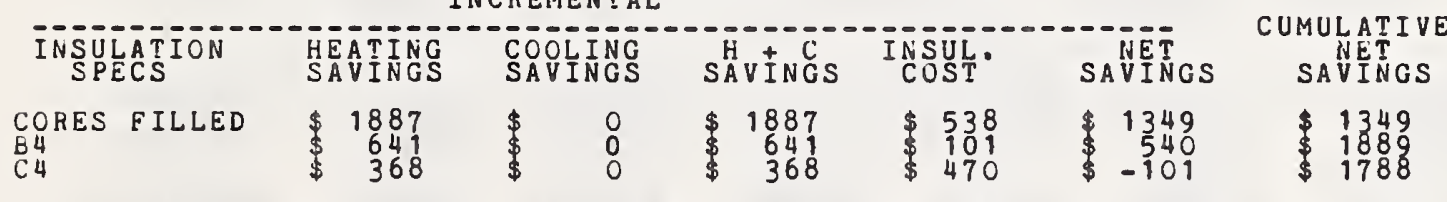

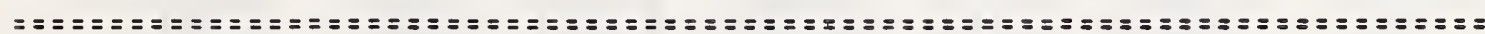

HEAT PUMP
ELECTRIC AIR COND. $\$ \$ \$ P 46 / K W H$

INCREMENTAL

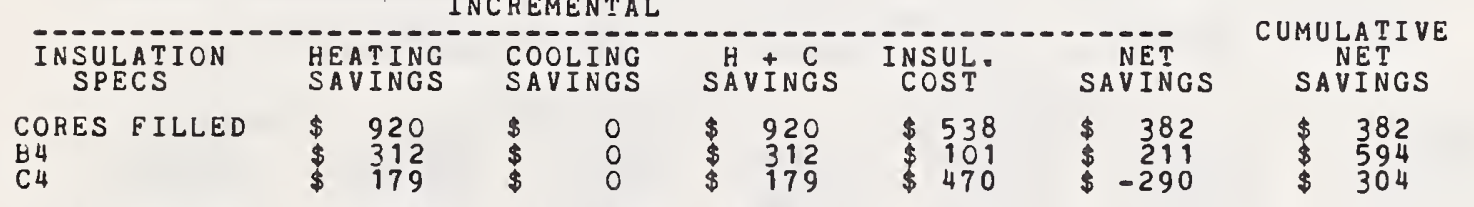


HUC 4

ALBUQUERGUE NEW MEXICO 200 SO FT HOUSE 8 IN RLOCK (PERLITE FILLED) COOLING LOADS BASED ON NO COOLING SAVINGS

\begin{tabular}{|c|c|c|c|c|}
\hline $\begin{array}{l}\text { INSULATION } \\
\text { SPECS }\end{array}$ & $\stackrel{U}{\text { VALUE }}$ & $\begin{array}{l}\text { INSUL } \\
\text { COST }\end{array}$ & $\begin{array}{l}\text { ANNUAL HTG } \\
\text { (MILLION BTU) }\end{array}$ & $\begin{array}{l}\text { ANNUAL CLG } \\
\text { (HILLION BTU) }\end{array}$ \\
\hline $\begin{array}{l}\text { BASE CASE } \\
\text { CORES FILLED } \\
\text { B4 } \\
\text { D4 } \\
\text { F5 }\end{array}$ & $\begin{array}{l}.249 \\
.141 \\
.104 \\
.099 \\
.071\end{array}$ & $\begin{array}{rr}\$ & 0 \\
\$ & 538 \\
\$ & 639 \\
\$ & 908 \\
\$ 1076\end{array}$ & $\begin{array}{l}22.407 \\
94.749 \\
12.186 \\
11.871 \\
10.137\end{array}$ & $\begin{array}{l}13.006 \\
13.006 \\
13: 006 \\
13.006 \\
13.006\end{array}$ \\
\hline $\begin{array}{l}\text { INSULATION } \\
\text { SPECS }\end{array}$ & INCREM ENTAL & $\begin{array}{c}\text { INCREHENTAL } \\
\text { COST }\end{array}$ & $\begin{array}{l}\text { INCREMENTAL } \\
\text { ANNUAL HTG }\end{array}$ & $\begin{array}{l}\text { INCFEMEITAL } \\
\text { ANNUAL CLG }\end{array}$ \\
\hline $\begin{array}{l}\text { CORES FILLED } \\
\text { B4 } \\
\text { D } 4 \\
\text { F5 }\end{array}$ & $\begin{array}{l}.108 \\
.037 \\
.005 \\
.028\end{array}$ & $\begin{array}{l}\$ 538 \\
\$ 101 \\
\$ 269 \\
\$ 168\end{array}$ & $\begin{array}{l}7.658 \\
2.563 \\
.315 \\
1.734\end{array}$ & $\begin{array}{l}.000 \\
.000 \\
.000 \\
.000\end{array}$ \\
\hline
\end{tabular}

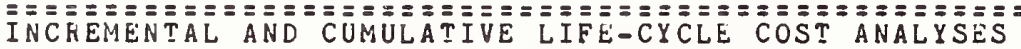

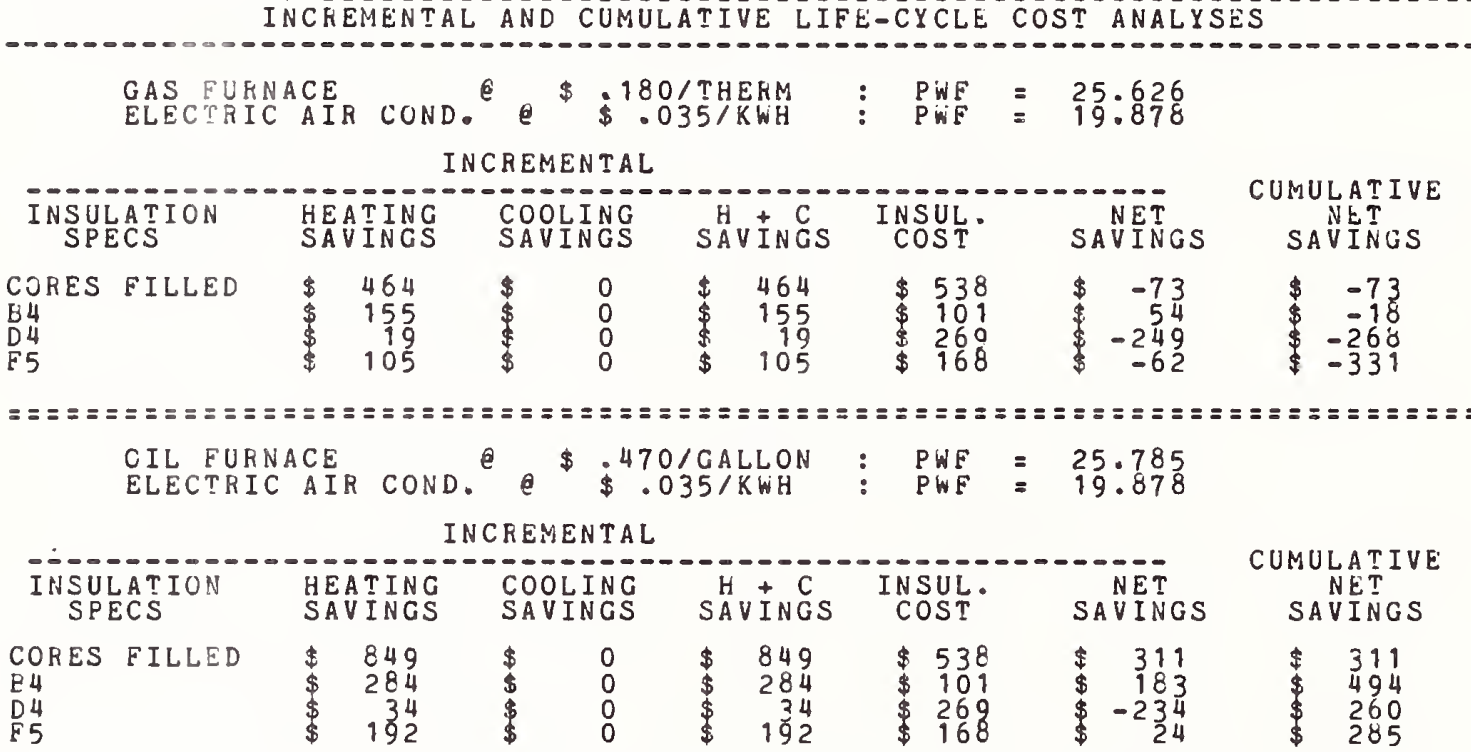

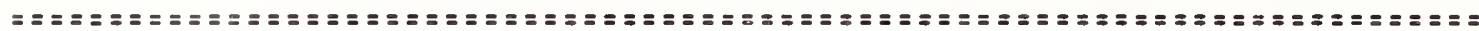

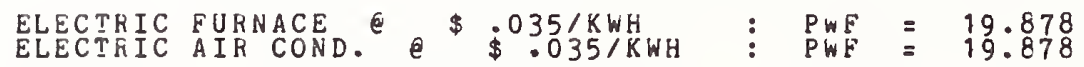

INCREMENT AL

\begin{tabular}{|c|c|c|c|c|c|c|}
\hline $\begin{array}{l}\text { IN SULATION } \\
\text { SPECS }\end{array}$ & $\begin{array}{l}\text { HEAT ING } \\
\text { SAVINGS }\end{array}$ & $\begin{array}{l}\text { COOLING } \\
\text { SAVINGS }\end{array}$ & $\begin{array}{l}\mathrm{H} \\
\text { SAVINGS }\end{array}$ & $\begin{array}{l}\text { INSUL. } \\
\text { COST }\end{array}$ & SAVINGS & $\begin{array}{c}\text { CUMULATIVE } \\
\text { NETT } \\
\text { SAVINGS }\end{array}$ \\
\hline $\begin{array}{l}\text { CORES FILLED } \\
\text { B } 4 \\
\text { D4 } \\
\text { F5 }\end{array}$ & $\begin{array}{rl} & 1561 \\
\$ & 522 \\
\$ & 64 \\
\$ & 353\end{array}$ & $\begin{array}{l}\$ \\
\$ \\
\$ \\
\$\end{array}$ & $\begin{array}{r}\$ 561 \\
\$ \quad 522 \\
\$ \quad 64 \\
\$ \quad 353\end{array}$ & $\begin{array}{l}\$ 538 \\
\$ 101 \\
\$ 269 \\
\$ 168\end{array}$ & $\begin{array}{r}1023 \\
\$ 421 \\
\$-204 \\
\$ 185\end{array}$ & $\begin{array}{l}\$ 1023 \\
\$ 1444 \\
\$ 1239 \\
\$ 1425\end{array}$ \\
\hline
\end{tabular}

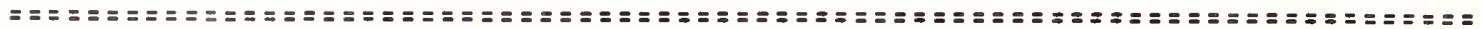

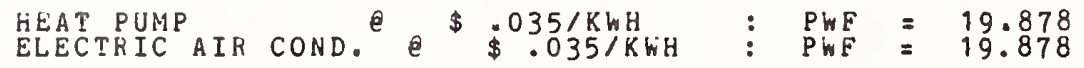

INCFEMENTAL

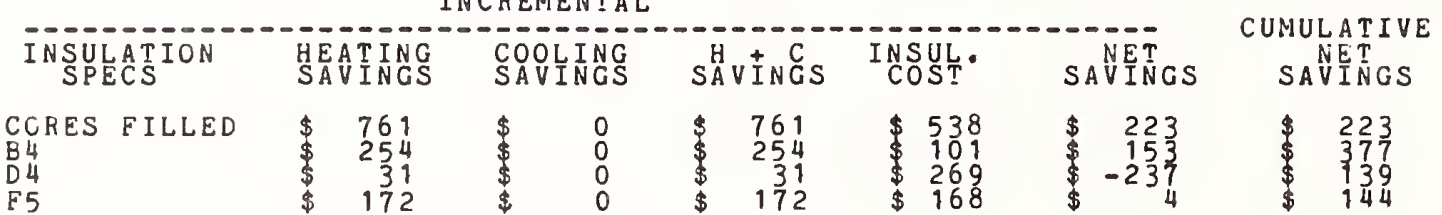

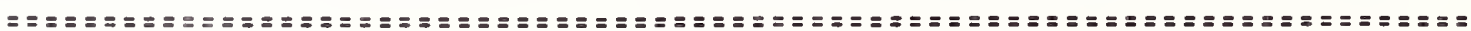


HUD4

ATLANTA GEORGIA ATLANTA GEORGIA 1200 SQ FT HOUSE IN BLOCK
COOLING LOADS BASED ON NO COOLING SAVINGS

8 IN BLOCK (PEKLITE FILLED)

\begin{tabular}{|c|c|c|c|c|}
\hline $\begin{array}{l}\text { INSULAT ION } \\
\text { SPECS }\end{array}$ & VALUE & $\begin{array}{l}\text { INSUL } \\
\text { COST }\end{array}$ & $\begin{array}{l}\text { ANNUAL }{ }^{H T G} \\
\text { MILLION }\end{array}$ & $\begin{array}{l}\text { ANNUAL CLG } \\
\text { (MILLION BTU }\end{array}$ \\
\hline $\begin{array}{l}\text { BASE CASE } \\
\text { CORES FILLED } \\
\text { B } 4 \\
\text { D4 } \\
\text { F5 }\end{array}$ & $\begin{array}{l}.249 \\
: 141 \\
1104 \\
: 099 \\
0 \\
071\end{array}$ & $\begin{array}{r}\$ 0 \\
538 \\
\$ 539 \\
\$ 908 \\
\$ 1076\end{array}$ & $\begin{array}{r}17.144 \\
12: 134 \\
10.461 \\
10.257 \\
9.141\end{array}$ & $\begin{array}{l}16.800 \\
16.800 \\
16.800 \\
16.800 \\
16.800\end{array}$ \\
\hline $\begin{array}{l}\text { INSULATION } \\
\text { SPECS }\end{array}$ & INCREM & $\begin{array}{c}\text { INCREMENTAL } \\
\text { COST }\end{array}$ & $\begin{array}{l}\text { INCKEMENTAL } \\
\text { ANNUAL HTG }\end{array}$ & $\begin{array}{l}\text { INCHENENTAL } \\
\text { ANLUAL CLG }\end{array}$ \\
\hline $\begin{array}{l}\text { CORES FILLED } \\
\text { B4 } \\
\text { D4 } \\
\text { F5 }\end{array}$ & $\begin{array}{l}.108 \\
.037 \\
0005 \\
.028\end{array}$ & $\begin{array}{l}\$ 538 \\
\$ 101 \\
\$ 269 \\
\$ 168\end{array}$ & $\begin{array}{l}5.010 \\
1.673 \\
1.204 \\
1.116\end{array}$ & $\begin{array}{l}.000 \\
.000 \\
.000 \\
.000\end{array}$ \\
\hline
\end{tabular}

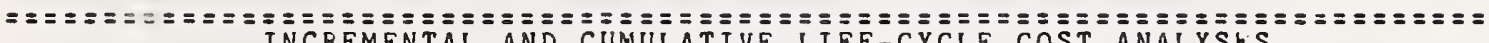
INCREMENTAL AND CUMULATIVE LIFE-CYCLE COST ANALYSES

OAS FURNAC

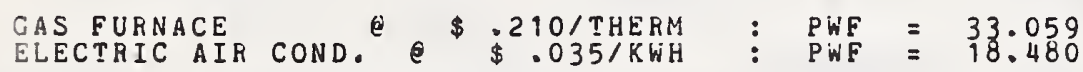

INCREMENTAL

\begin{tabular}{|c|c|c|c|c|c|c|}
\hline $\begin{array}{l}\text { INSULATION } \\
\text { SPECS }\end{array}$ & $\begin{array}{l}\text { HEATING } \\
\text { SAVINGS }\end{array}$ & $\begin{array}{l}\text { COOLING } \\
\text { SAVINGS }\end{array}$ & $\stackrel{H}{\text { SAVINC }}+\underset{C}{C}$ & $\begin{array}{l}\text { INSUL. } \\
\text { COST }\end{array}$ & $\begin{array}{l}\text { NET } \\
\text { SAVINGS }\end{array}$ & $\begin{array}{c}\text { CUMULATIVE } \\
\text { NET } \\
\text { SAVILCS }\end{array}$ \\
\hline $\begin{array}{l}\text { CORES FILLED } \\
\text { E4 } \\
04 \\
\text { E5 }\end{array}$ & $\begin{array}{ll}\$ & 457 \\
\$ & 152 \\
\$ & 18 \\
\$ & 101\end{array}$ & $\begin{array}{l}\$ \\
\$ \\
\$\end{array}$ & $\begin{array}{l}\quad 457 \\
\$ 152 \\
\quad 18 \\
\$ 101\end{array}$ & $\begin{array}{l}\$ 538 \\
\$ 101 \\
\$ 260 \\
\$ 168\end{array}$ & $\begin{array}{rr}\$ & -80 \\
\$ & 51 \\
\$ & -250 \\
\$ & -56\end{array}$ & $\begin{array}{r}-80 \\
-28 \\
-278 \\
-345\end{array}$ \\
\hline
\end{tabular}

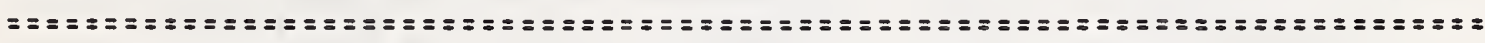

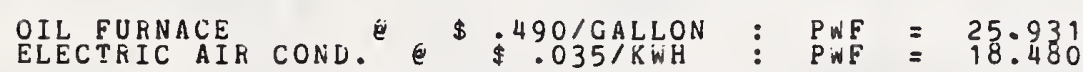
INCKEMENTAL

\begin{tabular}{|c|c|c|c|c|c|c|}
\hline $\begin{array}{l}\text { INSULATION } \\
\text { SPECS }\end{array}$ & $\begin{array}{l}\text { HEATING } \\
\text { SAVINGS }\end{array}$ & $\begin{array}{l}\text { COOLING } \\
\text { SAVINGS }\end{array}$ & $\begin{array}{l}H+C \\
\text { SAVINGS }\end{array}$ & $\begin{array}{l}\text { INSULT. } \\
\text { COST }\end{array}$ & $\begin{array}{l}\text { SAVINGS } \\
\text { SANG }\end{array}$ & $\begin{array}{c}\text { CUALLATIVE } \\
\text { SAVTETES }\end{array}$ \\
\hline $\begin{array}{l}\text { CORES FILLED } \\
\text { 4 } \\
04 \\
\text { F5 }\end{array}$ & $\begin{array}{ll}\$ & 582 \\
& 194 \\
\$ & 23 \\
\$ & 129\end{array}$ & $\begin{array}{l}\$ \\
\$ \\
\$ \\
\$\end{array}$ & $\begin{array}{ll}\$ & 582 \\
\$ & 194 \\
\$ & 23 \\
\$ & 129\end{array}$ & $\begin{array}{l}538 \\
\$ 101 \\
\$ 269 \\
\$ 168\end{array}$ & $\begin{array}{rr} & 44 \\
\$ & 93 \\
\$ & -245 \\
\$ & -38\end{array}$ & $\begin{array}{r}44 \\
138 \\
-107 \\
-145\end{array}$ \\
\hline
\end{tabular}

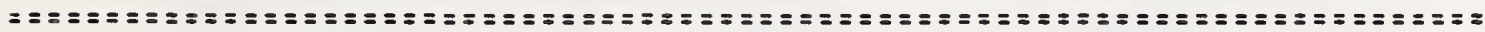
ELECTRIC FURNACE
ELECTRIC AIR COND. \$.035/KWH $\$$ PWF $=18.480$ INCREMENTAL

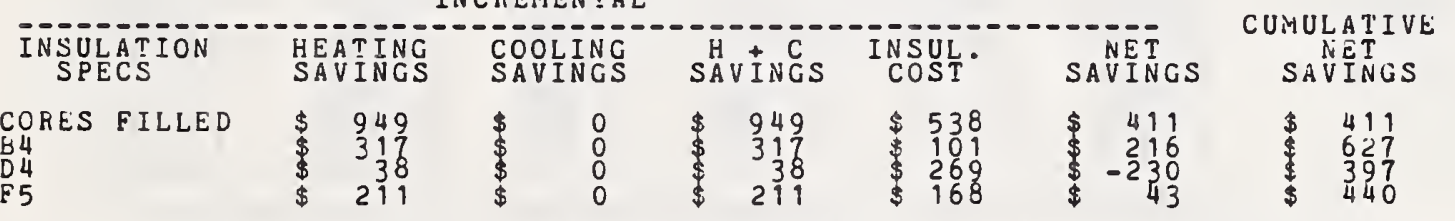

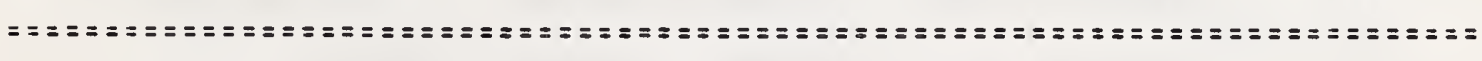
HEATPUMP
ELECTRIC AIR COND. e $\$ 035 / \mathrm{KWH}$

INCREMENTAL

\begin{tabular}{|c|c|c|c|c|c|c|}
\hline $\begin{array}{l}\text { INSULATION } \\
\text { SPECS }\end{array}$ & $\begin{array}{l}\text { HEATING } \\
\text { SAVINGS }\end{array}$ & $\begin{array}{l}\text { COOLING } \\
\text { SAVINGS }\end{array}$ & SÄVINGGS & $\begin{array}{l}\text { INSUL. } \\
\text { COST }\end{array}$ & SAVINGS & $\begin{array}{c}\text { CUMULATIVE } \\
\text { SET } \\
\text { SAVINGS }\end{array}$ \\
\hline KES FILLED & $\begin{array}{rr} & 441 \\
& 147 \\
& 17 \\
\$ & 98\end{array}$ & $\$$ & $\begin{array}{r}\quad 441 \\
\$ \quad 147 \\
\quad 17 \\
\$ \quad 98\end{array}$ & $\begin{array}{l}538 \\
\$ 101 \\
\$ 269 \\
\$ 168\end{array}$ & $\begin{array}{r}-96 \\
46 \\
-251 \\
-69\end{array}$ & $\begin{array}{r}-96 \\
-49 \\
-300 \\
-370\end{array}$ \\
\hline
\end{tabular}


HUD 4

IUDIANAPOLIS INDIAHA

8 IN BLOCK (PEKLITE FILLED)

1200 SQ ET HOUSE COOLING LOADS BASED ON NO COOLING SAVINGS

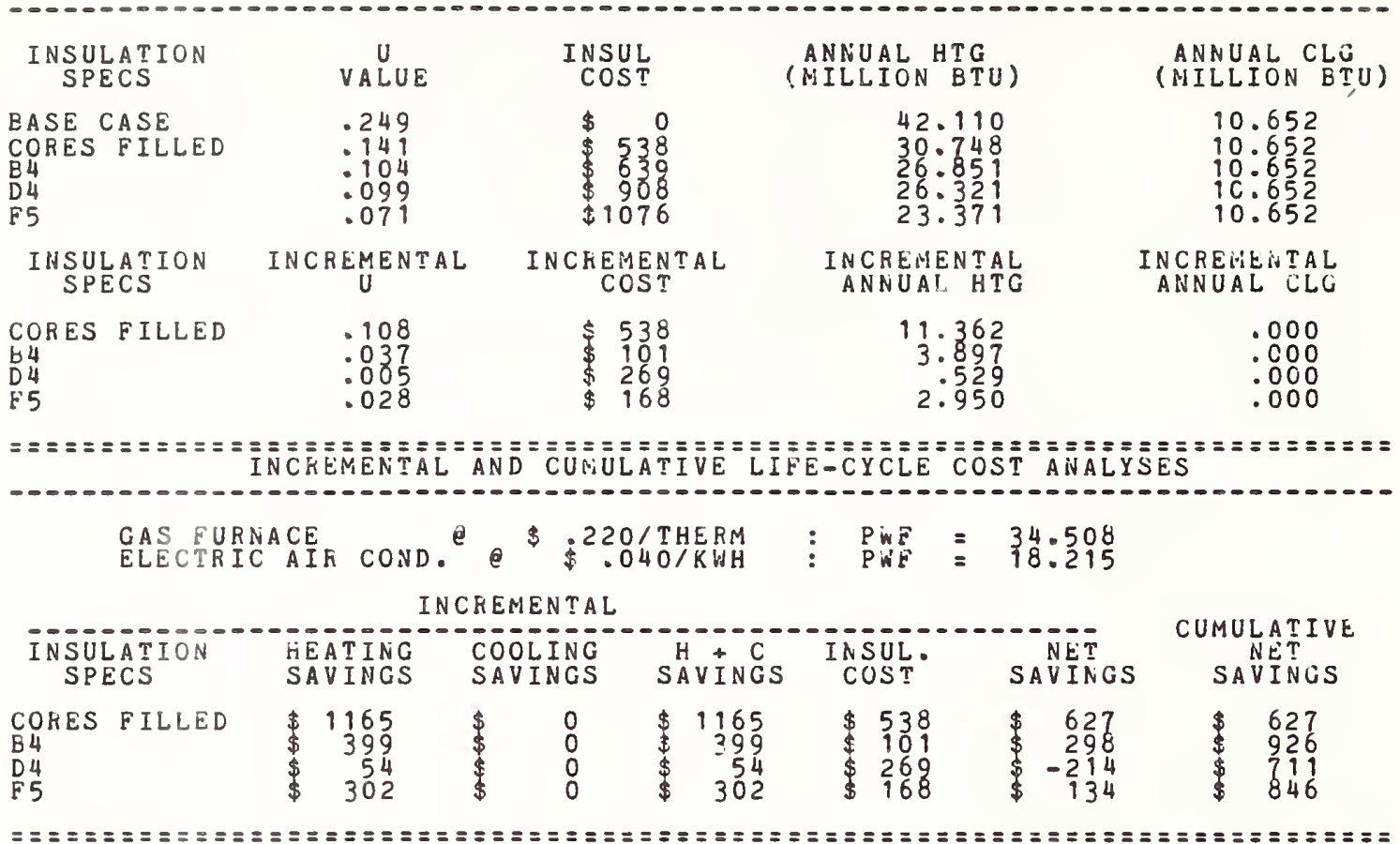

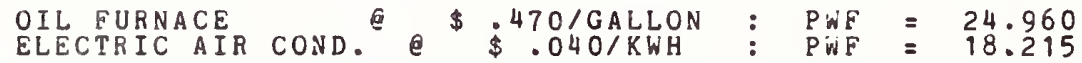

I NCREMENTAL

\begin{tabular}{|c|c|c|c|c|c|c|c|c|}
\hline $\begin{array}{l}\text { INSULAT ION } \\
\text { SPECS }\end{array}$ & $\begin{array}{l}\text { HEATING } \\
\text { SAVINGS }\end{array}$ & & GS & $\stackrel{H}{S A} \stackrel{+}{I} N G S^{C}$ & INSUL. & $S A L$ & $\begin{array}{l}\text { NET } \\
\text { VINGS }\end{array}$ & SAVITOS \\
\hline$\frac{\text { ORES FILLED }}{4}$ & $\begin{array}{r}1220 \\
\$ 198 \\
\$ 56 \\
\$ 316\end{array}$ & $\$$ & $\begin{array}{l}0 \\
0 \\
0 \\
0\end{array}$ & $\begin{array}{r}1220 \\
418 \\
\$ \quad 316 \\
\$ \quad 36\end{array}$ & $\begin{array}{l}\$ 538 \\
\$ 101 \\
\$ 269 \\
\$ 168\end{array}$ & $\$$ & $\begin{array}{r}682 \\
317 \\
-212 \\
148\end{array}$ & $\begin{array}{l}682 \\
\$ \quad 999 \\
\$ \\
\quad 787 \\
936\end{array}$ \\
\hline
\end{tabular}

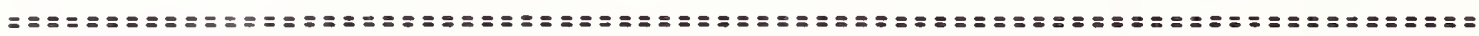
ELECTRIC FUKNACE e $\$ .040 / K W H$
ELECTKIC AIR COND. e $\$ .040 / K W H$ INCREMENTAL

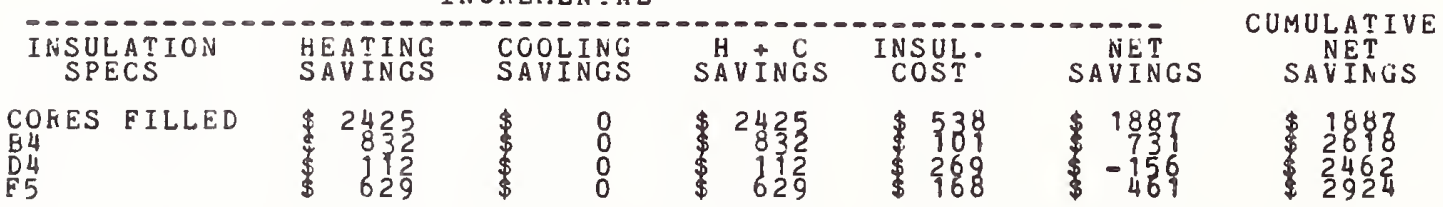

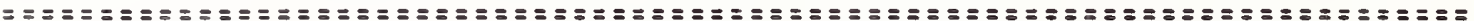
$\begin{array}{lll}\text { HEAT PUMP } & \text { e } \$ .040 / K W H & \text { PWF } \\ \text { ELECTRIC AIR COND. } & \$ 18.215\end{array}$

INCREMENTAL

\begin{tabular}{|c|c|c|c|c|c|c|c|c|}
\hline $\begin{array}{l}\text { NSULATION } \\
\text { SPECS }\end{array}$ & $\begin{array}{l}\text { HEATING } \\
\text { SAVINGS }\end{array}$ & & $\begin{array}{l}\text { ING } \\
\text { NGS }\end{array}$ & & $\begin{array}{c}+\underset{C}{\mathrm{I} N G S} \\
\end{array}$ & $\begin{array}{l}\text { INSUL. } \\
\text { COST }\end{array}$ & $\begin{array}{c}\text { NET } \\
\text { SAVINGS }\end{array}$ & $\begin{array}{c}\text { CUMULATIVE } \\
\text { NET } \\
\text { SAVINGS }\end{array}$ \\
\hline ES FILLED & $\begin{array}{r}1319 \\
\$ 449 \\
\$ \quad 69 \\
\$ \quad 340\end{array}$ & $\begin{array}{l}\$ \\
\$ \\
\$\end{array}$ & $\begin{array}{l}0 \\
0 \\
0 \\
0\end{array}$ & $\begin{array}{l}\$ \\
\$ \\
\$\end{array}$ & $\begin{array}{r}1311 \\
449 \\
61 \\
340\end{array}$ & $\begin{array}{l}538 \\
\$ 101 \\
\$ 269 \\
\$ 168\end{array}$ & $\begin{array}{rr}\$ & 773 \\
\$ & 348 \\
\$ & -207 \\
\$ & 172\end{array}$ & 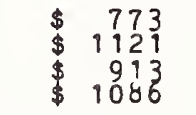 \\
\hline
\end{tabular}

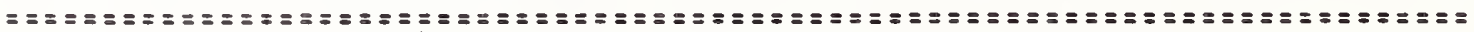


HUD 4

JACKSONVILLE FLORIDA 1200 SQ FT HOUSE 8 IN BLOCK (PERLITE FILLED) COOLING LOADS BASED ON NO COOLING SAVINGS

\begin{tabular}{|c|c|c|c|c|}
\hline $\begin{array}{l}\text { INSULATION } \\
\text { SPECS }\end{array}$ & $\begin{array}{l}\text { VALUE } \\
\text { VALU }\end{array}$ & $\begin{array}{l}\text { INSUL } \\
\text { COST }\end{array}$ & $\begin{array}{l}\text { ANNUAL HTG } \\
\text { (MILLION BTU) }\end{array}$ & $\begin{array}{l}\text { ANNUAL CLG } \\
\text { (MILLICN BTU) }\end{array}$ \\
\hline $\begin{array}{l}\text { BASE CASE } \\
\text { CORES FILLED } \\
\text { B } 4 \\
\text { D } 4 \\
\text { F } 5\end{array}$ & $\begin{array}{l}.249 \\
.141 \\
.104 \\
.099 \\
.071\end{array}$ & $\begin{array}{rr}\$ & 0 \\
\$ & 538 \\
\$ & 639 \\
\$ & 908 \\
\$ 1076\end{array}$ & $\begin{array}{l}3.608 \\
2.026 \\
1.538 \\
1.499 \\
1.296\end{array}$ & $\begin{array}{l}32 \cdot 704 \\
32 \cdot 704 \\
32.704 \\
32.704 \\
32.704\end{array}$ \\
\hline $\begin{array}{l}\text { INSULATION } \\
\text { SPECS }\end{array}$ & INCREM!ENTAL & $\begin{array}{c}\text { INCKEMENTAL } \\
\text { COST }\end{array}$ & $\begin{array}{l}\text { INCREMENTAL } \\
\text { ANNUAL HTG }\end{array}$ & $\begin{array}{l}\text { INCKEMEITTAL } \\
\text { ANNUAL CLG }\end{array}$ \\
\hline $\begin{array}{l}\text { CORES FILLED } \\
84 \\
04 \\
55\end{array}$ & $\begin{array}{l}108 \\
.037 \\
.005 \\
.028\end{array}$ & $\begin{array}{l}\$ 538 \\
\$ 101 \\
\$ 269 \\
\$ 168\end{array}$ & $\begin{array}{r}1.581 \\
.489 \\
.038 \\
.203\end{array}$ & $\begin{array}{l}.000 \\
.000 \\
.000 \\
.000\end{array}$ \\
\hline
\end{tabular}

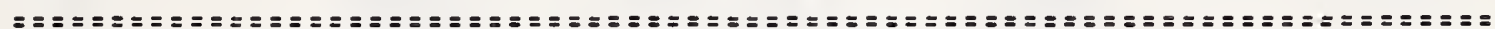
INCREMENTAL AND CUMULATIVE LIFE-CYCLE COST ANALYSES

- - - - - - - -

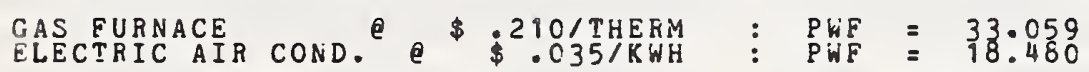

INCREMENTAL

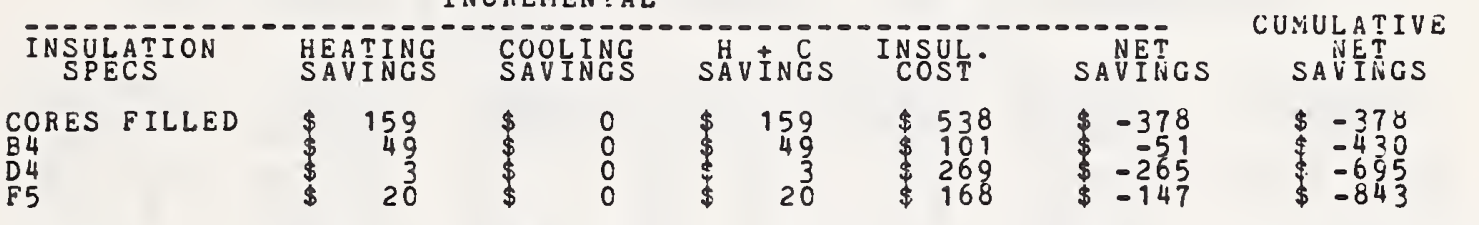

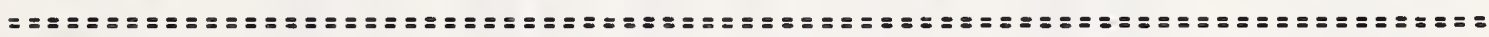

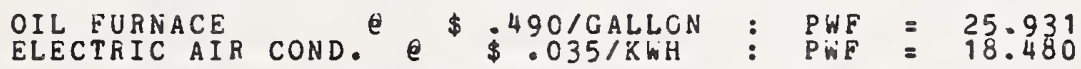

INCREMENTAL

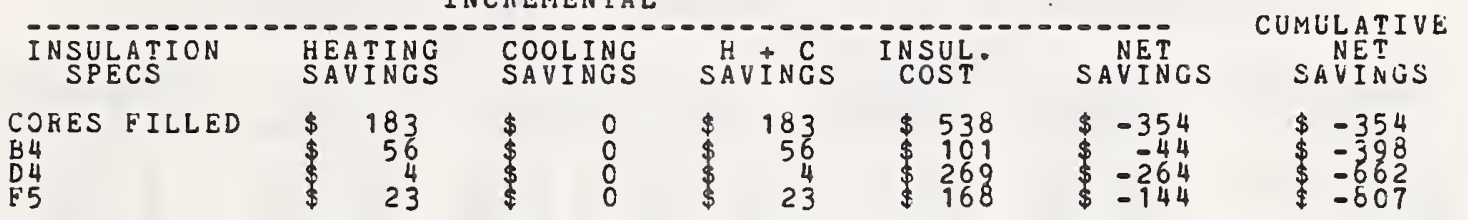

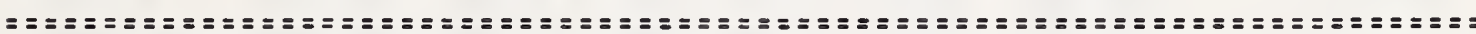

ELECTRIC FURNACE e $\$ .035 / K W H$
ELECTRIC AIRCOND. $\$$ PWF $=18.480$

INCREMENTAL

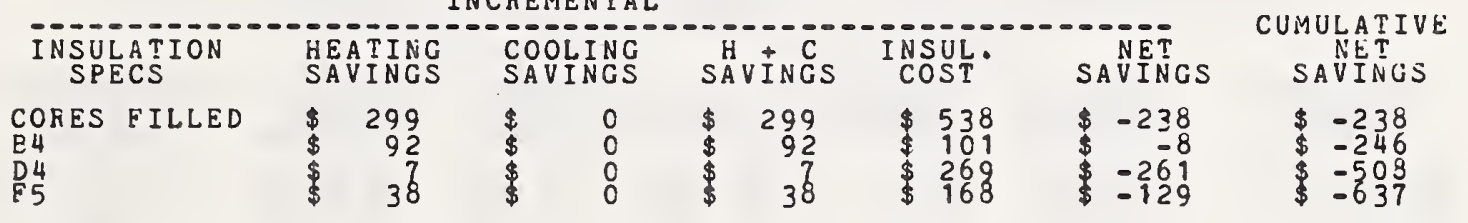

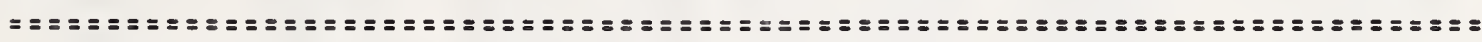
HEAT PUMP
ELECTRIC AIR COND. e $\$$ \$. $\$ 35 / K W H$

INCREMENTAL

\begin{tabular}{|c|c|c|c|c|c|c|c|c|}
\hline $\begin{array}{l}\text { INSULATION } \\
\text { SPECS }\end{array}$ & & I INGS & & & SAVINGS & $\begin{array}{l}\text { INSUL. } \\
\text { COST }\end{array}$ & $\begin{array}{l}\text { NAET } \\
\text { SAVINSS }\end{array}$ & $\begin{array}{c}\text { CURULATIVE } \\
\text { NETT } \\
\text { SAVINGS }\end{array}$ \\
\hline $\begin{array}{l}\text { CORES FILLED } \\
\text { B } 4 \\
\text { D4 } \\
\text { E5 }\end{array}$ & $\$$ & $\begin{array}{r}130 \\
40 \\
3 \\
16\end{array}$ & $\$$ & $\begin{array}{l}0 \\
0 \\
0 \\
0\end{array}$ & $\begin{array}{r}130 \\
40 \\
\$ \quad 3 \\
16\end{array}$ & $\begin{array}{l}538 \\
\$ 101 \\
\$ 269 \\
\$ 168\end{array}$ & $\begin{array}{r}-407 \\
\$-60 \\
\$-265 \\
\$-151\end{array}$ & $\begin{array}{l}\$-407 \\
\$-468 \\
-734 \\
-805\end{array}$ \\
\hline
\end{tabular}

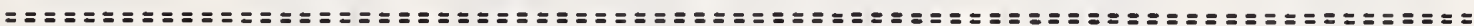


HUD 4 MADISON WISCONSIN 1200 SQ FT HOUSE 8 IN BLOCK (PERLITE FILLED)
COOLING LOADS BASED ON NO COOLING SAVINGS

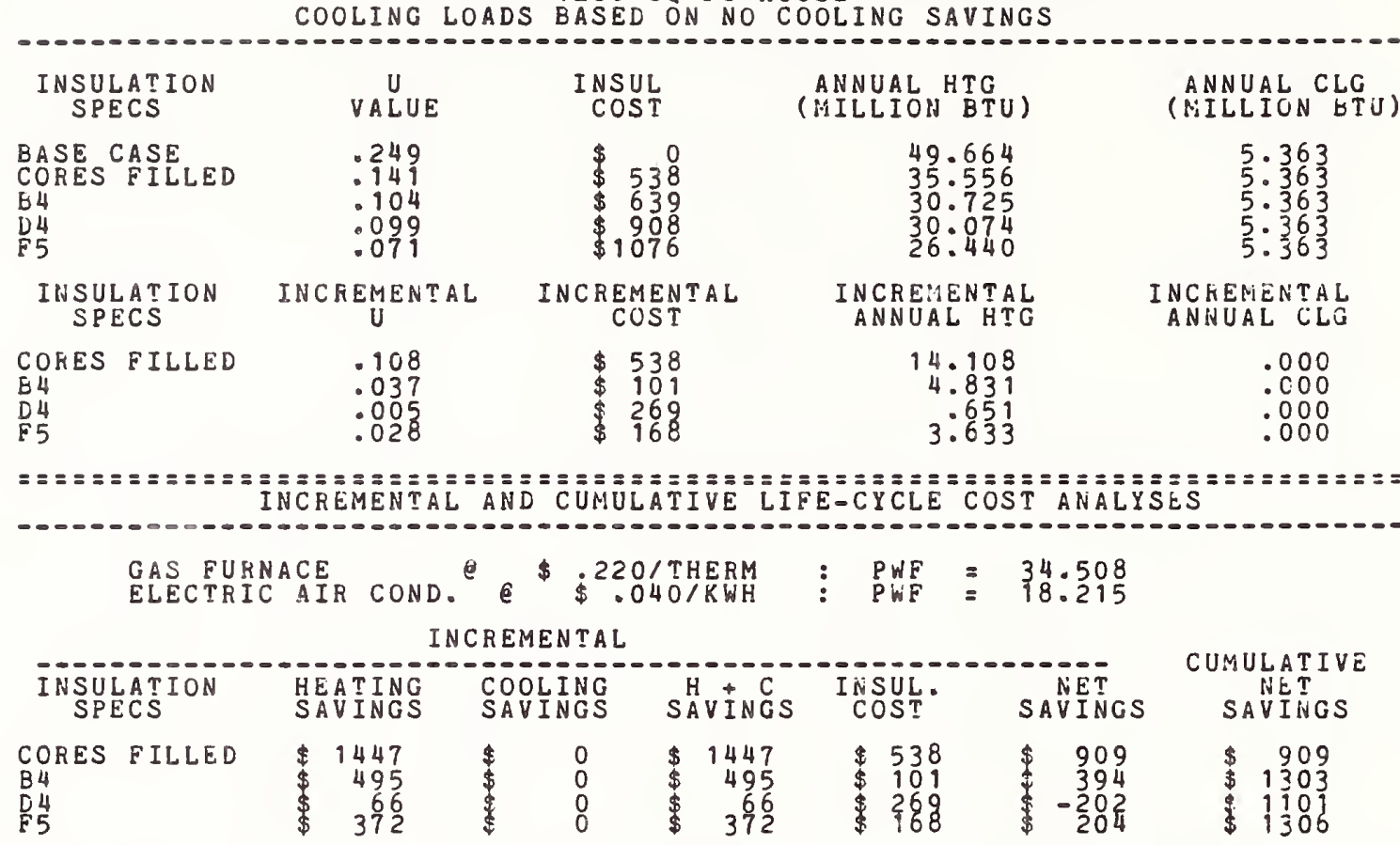

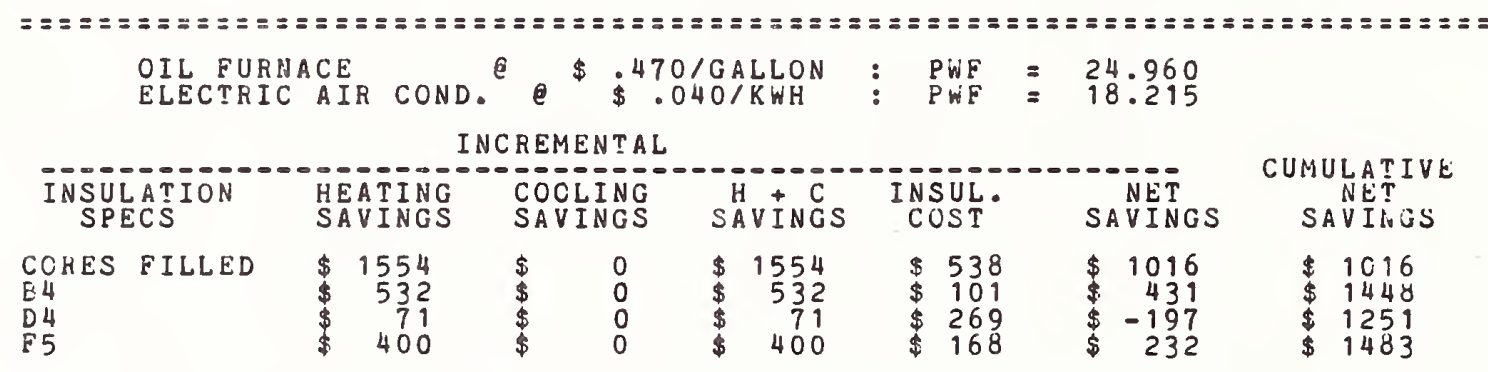

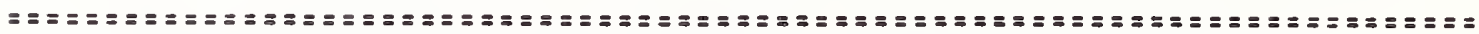

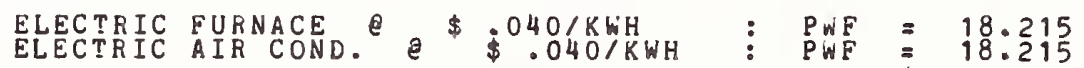

INCREMENTAL

\begin{tabular}{|c|c|c|c|c|c|c|}
\hline $\begin{array}{l}\text { INSULATION } \\
\text { SPECS }\end{array}$ & $\begin{array}{l}\text { HEATING } \\
\text { SAVINGS }\end{array}$ & $\begin{array}{l}\text { COOLING } \\
\text { SAVINGS }\end{array}$ & SAVINGS & $\begin{array}{l}\text { INSUL. } \\
\text { COST }\end{array}$ & $\begin{array}{l}\text { NET } \\
\text { SAVINGS }\end{array}$ & $\begin{array}{c}\text { CUNULATIVE } \\
\text { NEET } \\
\text { SAVINGS }\end{array}$ \\
\hline $\begin{array}{l}\text { COKES FILLED } \\
84 \\
\text { C4 } \\
\text { F5 }\end{array}$ & $\begin{array}{r}\$ 3011 \\
\$ 1031 \\
139 \\
\$ \quad 775\end{array}$ & $\begin{array}{l}\$ \\
\$\end{array}$ & $\begin{array}{r}3011 \\
\$ 1031 \\
139 \\
\$ \quad 775\end{array}$ & $\begin{array}{l}\$ 538 \\
\$ 101 \\
\$ 269 \\
\$ 168\end{array}$ & $\begin{array}{r}2473 \\
\$ 930 \\
\$-129 \\
\$ \quad 607\end{array}$ & $\begin{array}{l}2473 \\
3404 \\
3274 \\
3881\end{array}$ \\
\hline
\end{tabular}

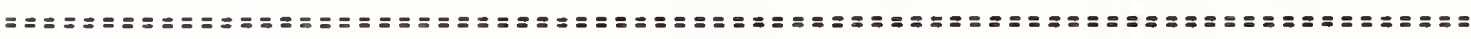

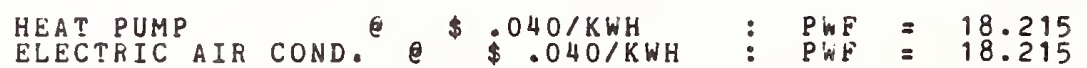

INCREMENTAL

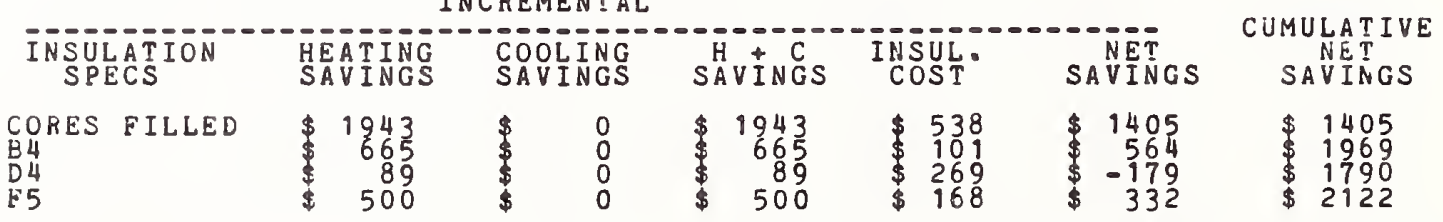


HUD 4

PHOENIX ARIZONA 200 SQ FT HOUSE IN BLOCK (PERLITE EILLED) COOLING LOADS BASED ON NO COOLING SAVINGS

\begin{tabular}{|c|c|c|c|c|}
\hline $\begin{array}{l}\text { INSULATION } \\
\text { SPECS }\end{array}$ & VALUE & $\begin{array}{l}\text { INSUL } \\
\text { COST }\end{array}$ & $\begin{array}{l}\text { ANNUAL HTG } \\
\text { (MILLION }\end{array}$ & $\begin{array}{l}\text { ANNUAL CLG } \\
\text { (MILLION BTU) }\end{array}$ \\
\hline $\begin{array}{l}\text { BASE CASE } \\
\text { CORES FILLED } \\
\text { B } 4 \\
\text { C4 } \\
\text { E5 }\end{array}$ & $\begin{array}{l}.249 \\
.149 \\
.104 \\
.099 \\
.071\end{array}$ & $\begin{array}{r}\$ 0 \\
\$ 538 \\
\$ 639 \\
\$ 908 \\
\$ 1076\end{array}$ & $\begin{array}{l}4.055 \\
2.233 \\
1.680 \\
1.642 \\
1.445\end{array}$ & $\begin{array}{l}42.003 \\
42.003 \\
42.003 \\
42.003 \\
42.003\end{array}$ \\
\hline $\begin{array}{l}\text { IHSULATION } \\
\text { SPECS }\end{array}$ & INCKEMENTAL & $\begin{array}{c}\text { INCREMENTAL } \\
\text { COST }\end{array}$ & $\begin{array}{l}\text { INCREMENTAL } \\
\text { ANNUAL HTG }\end{array}$ & $\begin{array}{l}\text { INCKEMENTAL } \\
\text { ANNUAL CLG }\end{array}$ \\
\hline $\begin{array}{l}\text { CORES FILLED } \\
\text { E } 4 \\
\text { D } 4 \\
\text { F5 }\end{array}$ & $\begin{array}{l}.108 \\
.037 \\
.005 \\
.028\end{array}$ & $\begin{array}{l}\$ 338 \\
\$ 101 \\
\$ 269 \\
\$ 168\end{array}$ & $\begin{array}{r}1.822 \\
.553 \\
.038 \\
.197\end{array}$ & $\begin{array}{l}.000 \\
.000 \\
.000 \\
.000\end{array}$ \\
\hline
\end{tabular}

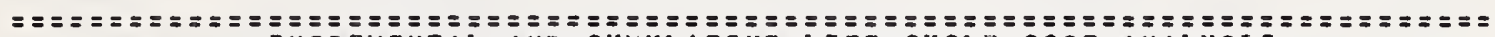
INCREMENTAL AND CUMULATIVE LIFE-CYCLE COST ANALYSES

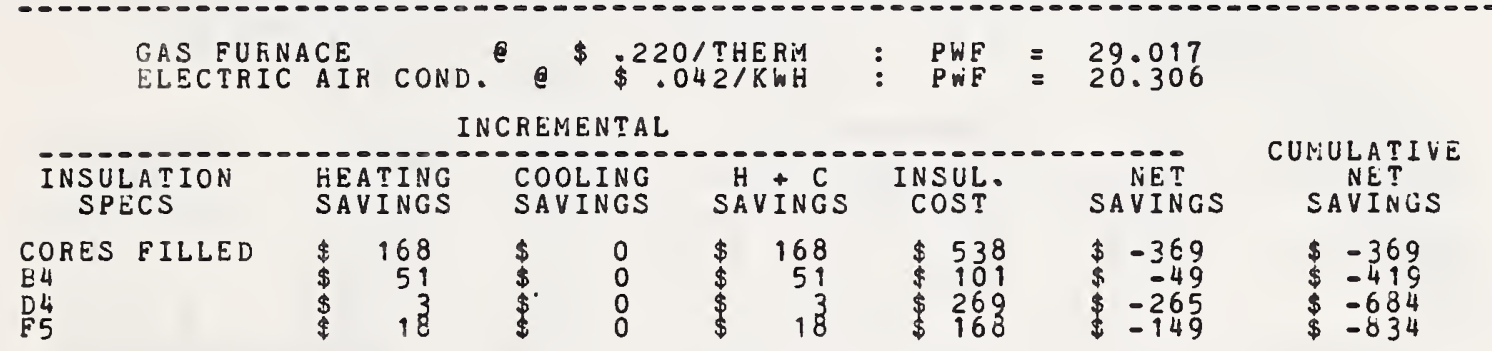

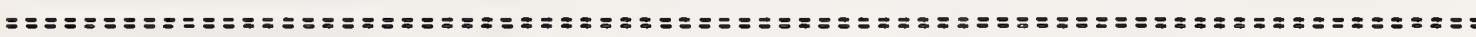

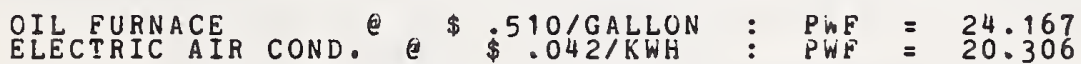

INCREMENTAL

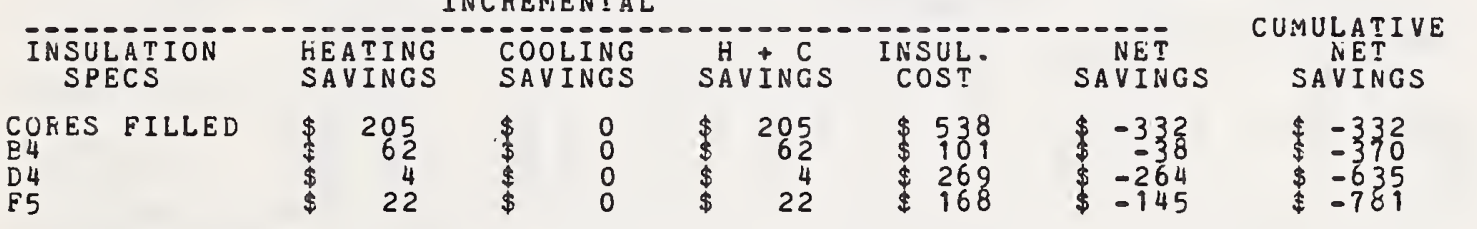

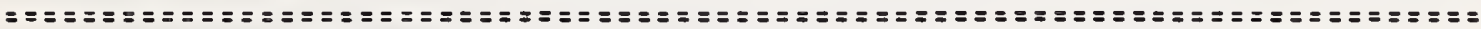

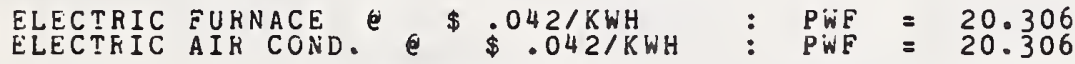

INCKEMENTAL

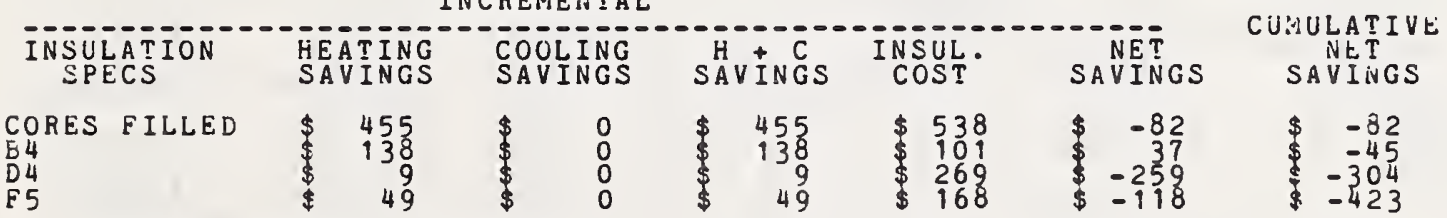

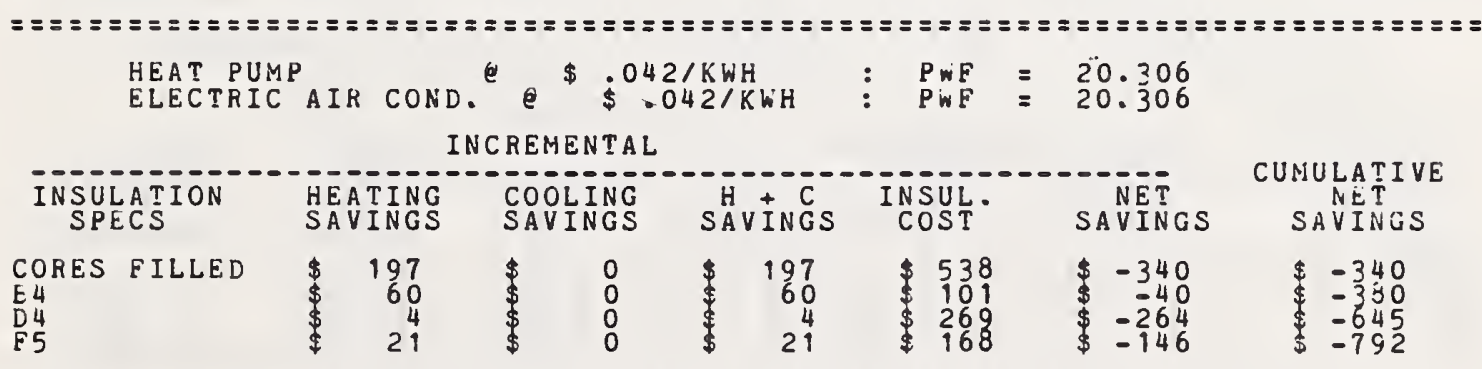

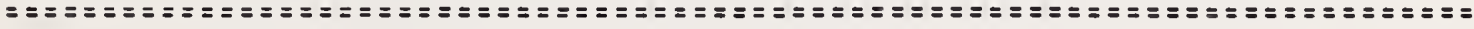


HUD 4

TAMPA FLOHIDA 1200 SO FT HOUSE 8 IN BLOCK (PERLITE FILLED) COOLING LOADS BASED ON NO COOLING SAVINGS

\begin{tabular}{|c|c|c|c|c|}
\hline $\begin{array}{l}\text { INSULATION } \\
\text { SPECS }\end{array}$ & VALUE & INSUL & (MNLUAL HTG & $\begin{array}{l}\text { ANNUAL CLG } \\
\text { (MILION BTU) }\end{array}$ \\
\hline $\begin{array}{l}\text { BASE CASE } \\
\text { CORES FILLED } \\
\text { B } 4 \\
\text { D } 4 \\
\text { F5 }\end{array}$ & $\begin{array}{l}.249 \\
.141 \\
: 104 \\
.099 \\
.071\end{array}$ & $\begin{array}{rr}\$ & 0 \\
\$ & 538 \\
\$ & 639 \\
\$ & 908 \\
\$ 1076\end{array}$ & $\begin{array}{l}.854 \\
.415 \\
.281 \\
.271 \\
.223\end{array}$ & $\begin{array}{l}38: 162 \\
38: 162 \\
38: 162 \\
38: 162 \\
38: 162\end{array}$ \\
\hline $\begin{array}{l}\text { INSULATION } \\
\text { SPECS }\end{array}$ & INCREMENTAL & $\begin{array}{l}\text { INCREMENTAL } \\
\text { COST }\end{array}$ & $\begin{array}{l}\text { INCREMENTAL } \\
\text { ANNUAL HTC }\end{array}$ & $\begin{array}{l}\text { INCREMENTAL } \\
\text { ANNUAL CLG }\end{array}$ \\
\hline $\begin{array}{l}\text { COKES FILLED } \\
\text { H } 4 \\
\text { D } 4 \\
\text { F5 }\end{array}$ & $\begin{array}{l}.108 \\
.037 \\
.005 \\
.028\end{array}$ & $\begin{array}{l}538 \\
\$ 101 \\
\$ 269 \\
\$ 168\end{array}$ & $\begin{array}{l}.439 \\
.134 \\
.010 \\
.048\end{array}$ & $\begin{array}{l}.000 \\
.000 \\
.000 \\
.000\end{array}$ \\
\hline \multicolumn{5}{|c|}{ 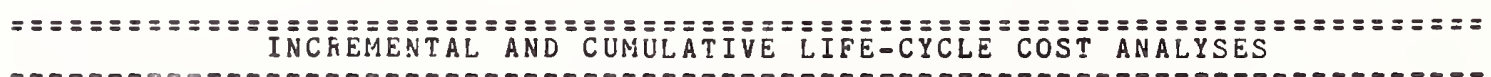 } \\
\hline
\end{tabular}

$$
\text { INCREMENTAL }
$$

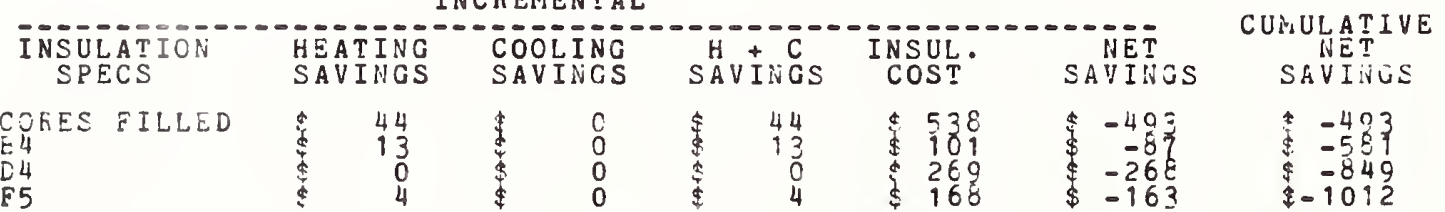

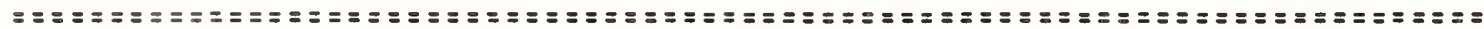

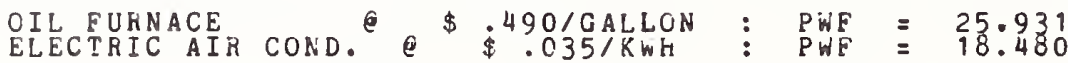

INCREMENTAL

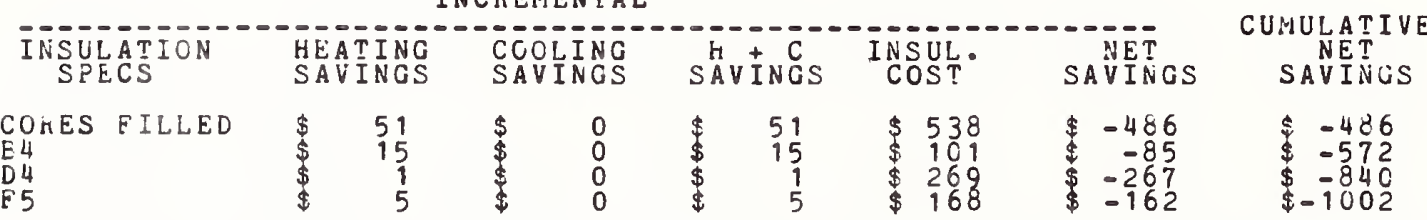

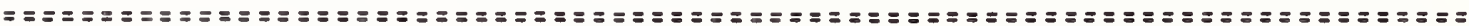

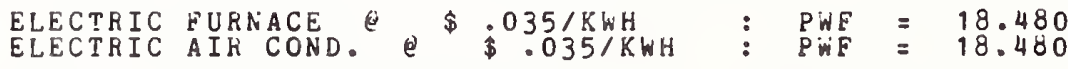

INCREMENTAL

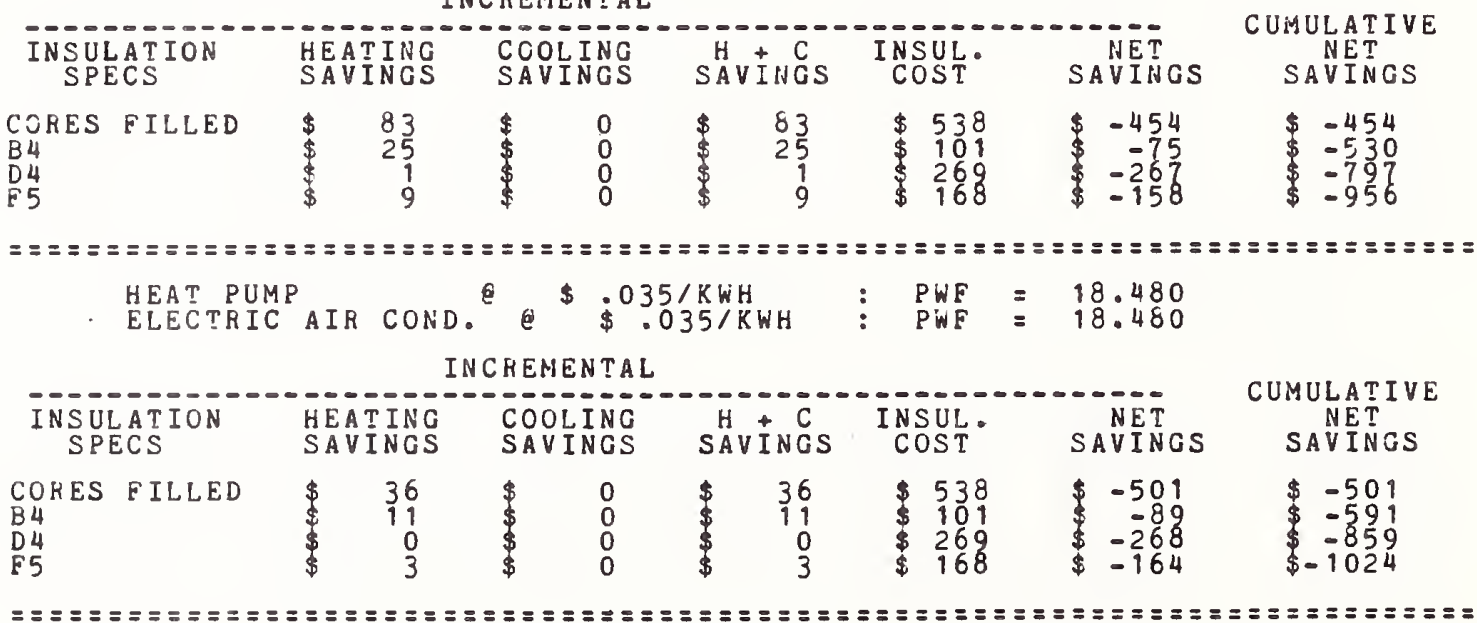


HUD 4

WASHINGTON D.C

8 IN BLOCK (PEKLITE FILLED) COOLING LOADS BASED ON NO COOLING SAVINGS

\begin{tabular}{|c|c|c|c|c|}
\hline $\begin{array}{l}\text { INSULATION } \\
\text { SPECS }\end{array}$ & VALUE & $\begin{array}{l}\text { INSUL } \\
\text { COST }\end{array}$ & $\begin{array}{l}\text { ANNUAL HTG } \\
\text { (MILLION BTU) }\end{array}$ & (ANNUAL CLG \\
\hline $\begin{array}{l}\text { EASE CASE } \\
\text { COKES FILLED } \\
\text { B } 4 \\
\text { D4 } \\
\text { F5 }\end{array}$ & $\begin{array}{l}.249 \\
.149 \\
.104 \\
.099 \\
.071\end{array}$ & $\begin{array}{rr} & 0 \\
& 538 \\
639 \\
\$ 908 \\
91076\end{array}$ & $\begin{array}{l}22.035 \\
14.589 \\
12.058 \\
11.726 \\
9.904\end{array}$ & $\begin{array}{l}15.674 \\
15.674 \\
15.674 \\
15.674 \\
15.674\end{array}$ \\
\hline $\begin{array}{l}\text { INSULATION } \\
\text { SPECS }\end{array}$ & INCREMENTAL & $\begin{array}{c}\text { INCREMENTAL } \\
\text { COST }\end{array}$ & $\begin{array}{l}\text { INCREMENTAL } \\
\text { ANNUAL HTG }\end{array}$ & $\begin{array}{l}\text { INCHENENTAL } \\
\text { ANNUAL CLC }\end{array}$ \\
\hline $\begin{array}{l}\text { CORES FILLED } \\
\text { B4 } \\
\text { D } 4 \\
15\end{array}$ & $\begin{array}{l}.108 \\
.037 \\
.005 \\
.028\end{array}$ & $\begin{array}{l}\$ 538 \\
\$ 101 \\
\$ 269 \\
\$ 168\end{array}$ & $\begin{array}{r}7.446 \\
2.531 \\
1.332 \\
1.822\end{array}$ & $\begin{array}{l}.000 \\
.000 \\
.000 \\
.000\end{array}$ \\
\hline
\end{tabular}

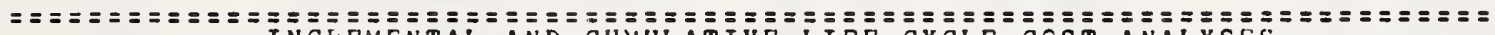
INCKEMENTAL AND CUMULATIVE LIFE-CYCLE COST ANALYSES

CAS FURNACE
ELECTRICAIF COND.

INCREMENTAL

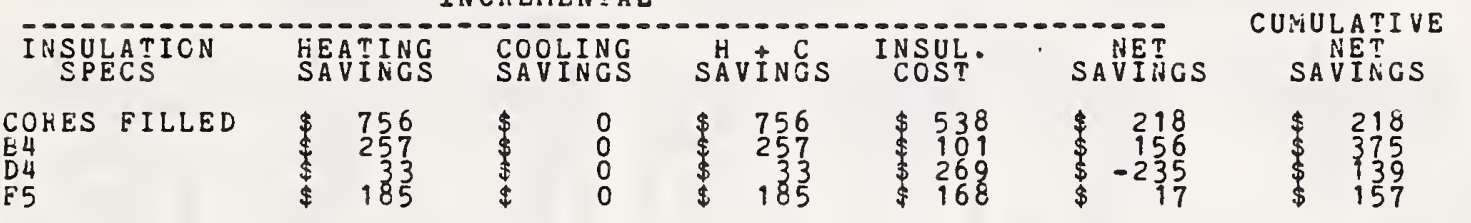

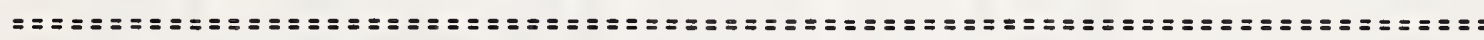

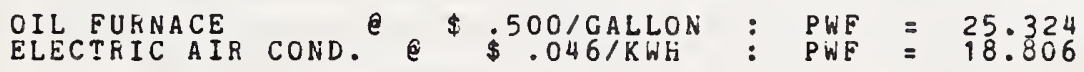

INCREMENTAL

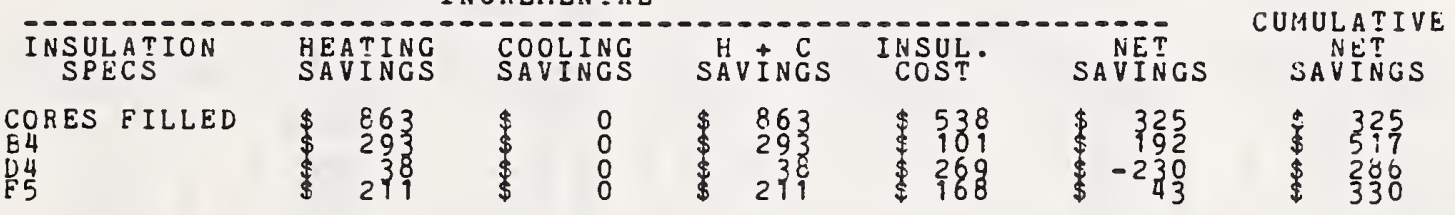

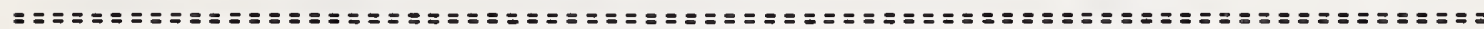
ELECTRIC FURNACE $e$ \$ $\$ .046 / \mathrm{KWH}$
ELECTRIC AIK COND. $\$$ PWF $=18.806$

INCREMENTAL

\begin{tabular}{|c|c|c|c|c|c|c|c|}
\hline $\begin{array}{l}\text { INSULATION } \\
\text { SPECS }\end{array}$ & $\begin{array}{l}\text { HEATING } \\
\text { SAVINGS }\end{array}$ & & $\begin{array}{l}\text { I NG } \\
\text { NGS }\end{array}$ & $\stackrel{H}{\text { SAV I INGS }} \underset{\text { C }}{C}$ & $\begin{array}{l}\text { INSUL. } \\
\text { COST }\end{array}$ & SAVINGS & $\begin{array}{l}\text { CUMULATIVE } \\
\text { ViVT } \\
\text { SAVINGS }\end{array}$ \\
\hline$\frac{4}{4} 45 E S$ FILLED & 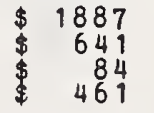 & $\begin{array}{l}\$ \\
\$ \\
\$\end{array}$ & $\begin{array}{l}0 \\
0 \\
0 \\
0\end{array}$ & $\begin{array}{r}1887 \\
641 \\
\$ 84 \\
461\end{array}$ & $\begin{array}{l}\$ 538 \\
\$ 101 \\
\$ 269 \\
\$ 168\end{array}$ & $\begin{array}{r}1349 \\
\$ 540 \\
\$-184 \\
\$ 293\end{array}$ & $\begin{array}{l}1349 \\
\$ 1889 \\
\$ 1704 \\
\$ 1998\end{array}$ \\
\hline
\end{tabular}

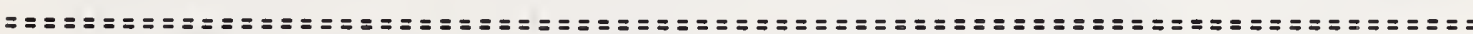
HEATPUMP
ELECTRIC AIR COND.

INCREMENTAL

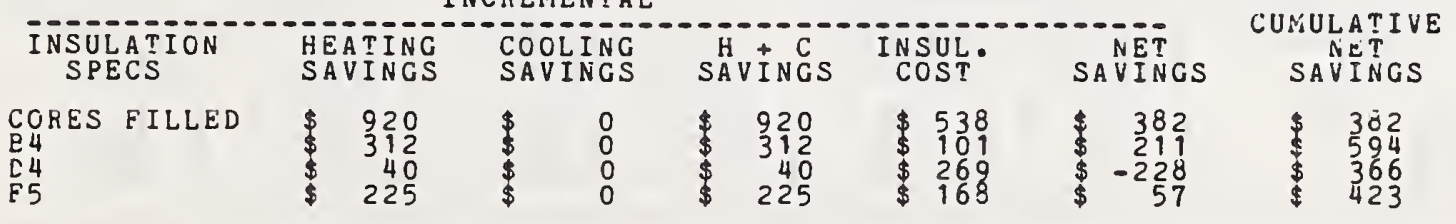

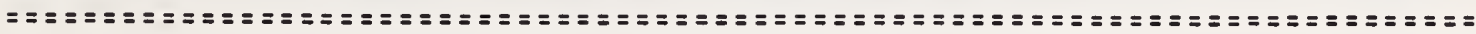


HUD5

ALBUQUERQUE NEW MEXICO 200 SO ET HOUSE HOOD FRAME WALL BASED ON NO COOLING SAVINGS

\begin{tabular}{|c|c|c|c|c|}
\hline $\begin{array}{l}\text { INSULATION } \\
\text { SPECS }\end{array}$ & VALUE & $\begin{array}{l}\text { INSUL } \\
\text { COST }\end{array}$ & $\begin{array}{l}\text { ANNUAL HTG } \\
\text { (MILLION BTU) }\end{array}$ & $\begin{array}{l}\text { ANNUAL CLG } \\
\text { (MILLION BTU) }\end{array}$ \\
\hline $\begin{array}{l}\text { BASE CASE } \\
R=11 \\
R=13 \\
R=19\end{array}$ & $\begin{array}{l}.218 \\
.082 \\
.076 \\
.055\end{array}$ & $\begin{array}{l}\$ \\
\$ \\
213 \\
280 \\
\$ 504\end{array}$ & $\begin{array}{r}20.869 \\
11.386 \\
10.966 \\
9.482\end{array}$ & $\begin{array}{l}14.517 \\
14.517 \\
14.517 \\
14.517\end{array}$ \\
\hline $\begin{array}{l}\text { INSULATION } \\
\text { SPECS }\end{array}$ & INCREMENTAL & $\begin{array}{c}\text { INCREMENTAL } \\
\text { COST }\end{array}$ & $\begin{array}{l}\text { INCREMENTAL } \\
\text { ANNUAL HTG }\end{array}$ & $\begin{array}{l}\text { INCREMENTAL } \\
\text { ANNUAL CLG }\end{array}$ \\
\hline $\begin{array}{l}R=11 \\
R=13 \\
R=19\end{array}$ & $\begin{array}{r}.136 \\
.006 \\
.021\end{array}$ & $\begin{array}{r}213 \\
\$ \quad 67 \\
\$ 224\end{array}$ & $\begin{array}{r}9.483 \\
.420 \\
1.483\end{array}$ & $\begin{array}{l}.000 \\
.000 \\
.000\end{array}$ \\
\hline
\end{tabular}

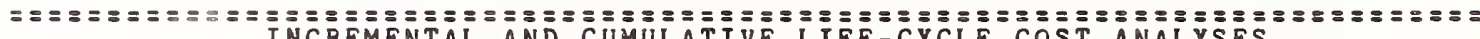
INCREMENTAL AND CUMULATIVE LIFE-CYCLE COST ANALYSES

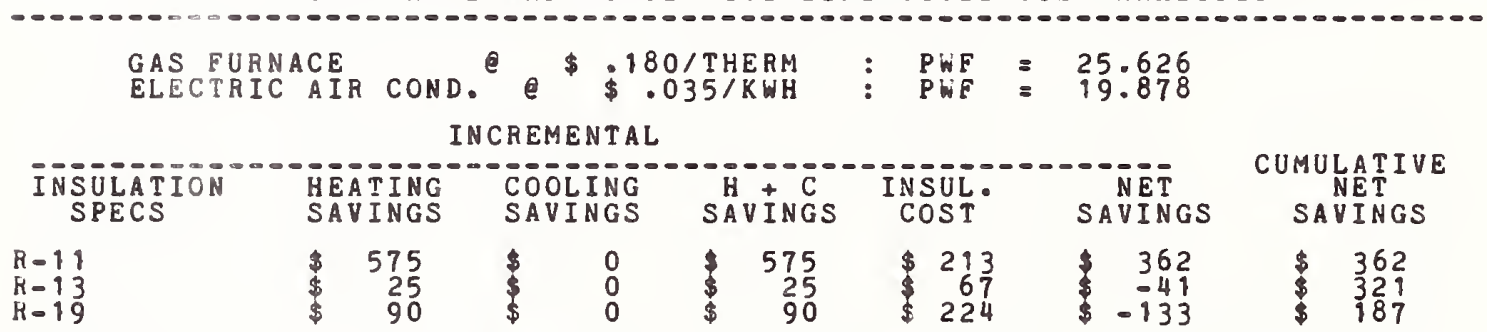

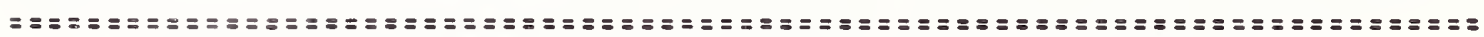

OILCEURACE

INCREMENTAL

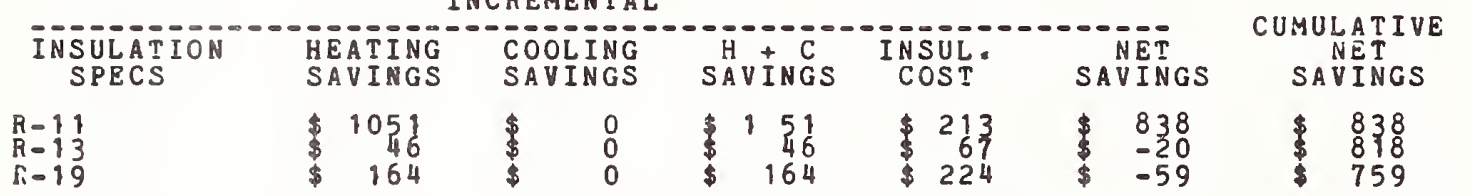

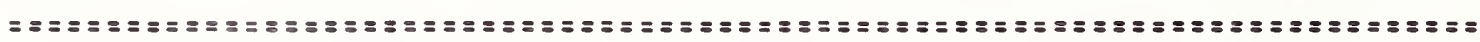

ELECTRIC FURNACE e
ELECTRIC AIRCOND. $\$ 035 / K W H$

INCREMENTAL

\begin{tabular}{|c|c|c|c|c|c|c|}
\hline $\begin{array}{l}\text { NSULAT ION } \\
\text { SPECS }\end{array}$ & $\begin{array}{l}\text { HEATING } \\
\text { SAVINGS }\end{array}$ & $\begin{array}{l}\text { COOLING } \\
\text { SAVINGS }\end{array}$ & SAVINGS & $\begin{array}{l}\text { INSUL. } \\
\text { COST }\end{array}$ & SAVINGS & $\begin{array}{c}\text { CUMULATIVE } \\
\text { NETT } \\
\text { SAVINGS }\end{array}$ \\
\hline 13 & $\begin{array}{r}1933 \\
85 \\
302\end{array}$ & $\$$ & $\begin{array}{r}1933 \\
\$ 85 \\
302\end{array}$ & $\begin{array}{l}213 \\
67 \\
224\end{array}$ & $\begin{array}{r}1720 \\
18 \\
\quad 78\end{array}$ & $\begin{array}{l}1720 \\
1738 \\
11817\end{array}$ \\
\hline
\end{tabular}

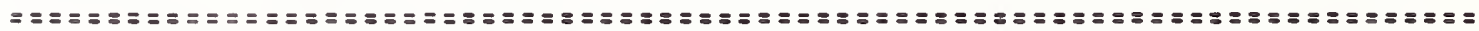

HEATPUMP

INCREMENTAL

\begin{tabular}{|c|c|c|c|c|c|c|c|}
\hline $\begin{array}{l}\text { INSULATION } \\
\text { SPECS }\end{array}$ & $\begin{array}{l}\text { HEATING } \\
\text { SAVINGS }\end{array}$ & $\begin{array}{l}\text { COOLING } \\
\text { SAVINGS }\end{array}$ & $\begin{array}{l}H+C \\
\text { SAVINGS }\end{array}$ & $\begin{array}{l}\text { INSUL } \\
\text { COST }\end{array}$ & & $\begin{array}{l}\text { VET } \\
\text { INGS }\end{array}$ & $\begin{array}{l}\text { CUMULATIVE } \\
\text { NET } \\
\text { SAVINGS }\end{array}$ \\
\hline 13 & $\begin{array}{r}942 \\
\quad \$ 17 \\
147\end{array}$ & $\$$ & $\begin{array}{r}942 \\
41 \\
147\end{array}$ & $\begin{array}{r}213 \\
\$ 27 \\
224\end{array}$ & $\$$ & $\begin{array}{l}729 \\
-25 \\
-76\end{array}$ & $\begin{array}{l}729 \\
704 \\
628\end{array}$ \\
\hline
\end{tabular}

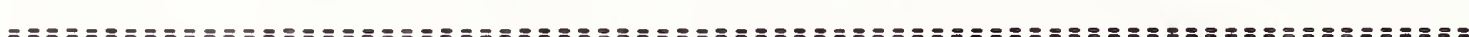


HUD5

ATLANTA GEORGIA 200 . SO FT HOUSE WOOD FRAME WALL COOLING LOADS BASED ON FT HOUSE COOLING SAVINGS

\begin{tabular}{|c|c|c|c|c|}
\hline INSULATION & VALUE & INSUL & (MINLAL HTONG & $\begin{array}{l}\text { ANNUAL CLG } \\
\text { (MILLION BTU) }\end{array}$ \\
\hline $\begin{array}{l}\text { BASE CASE } \\
R=11 \\
R=13 \\
R=19\end{array}$ & $\begin{array}{r}.218 \\
.082 \\
.876 \\
.055\end{array}$ & $\begin{array}{r}\$ \\
\$ \\
213 \\
280 \\
504\end{array}$ & $\begin{array}{r}16.266 \\
18: 1826 \\
8.841\end{array}$ & $\begin{array}{l}17.889 \\
17.8889 \\
17.889\end{array}$ \\
\hline $\begin{array}{l}\text { INSULATION } \\
\text { SPECS }\end{array}$ & INCREM UENTAL & $\begin{array}{c}\text { INCREMENTAL } \\
\text { COST }\end{array}$ & $\begin{array}{l}\text { INCREMENTAL } \\
\text { ANNUAL HTG }\end{array}$ & $\begin{array}{l}\text { INCREMENTAL } \\
\text { AHIUAL CLG }\end{array}$ \\
\hline $\begin{array}{l}R-11 \\
R-13 \\
R-19\end{array}$ & $\begin{array}{r}136 \\
: 006 \\
.021\end{array}$ & $\begin{array}{l}\$ 213 \\
\$ 224\end{array}$ & $\begin{array}{r}6.165 \\
: 275 \\
.985\end{array}$ & $\begin{array}{l}.000 \\
.000 \\
.000\end{array}$ \\
\hline
\end{tabular}

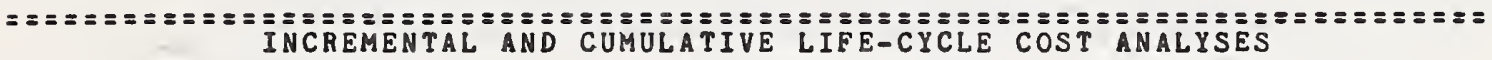

GAS FURNACE
ELECTRICAIR COND. $e^{\$ 210 / T H E R M ~} \$$ PWF INCREMENTAL

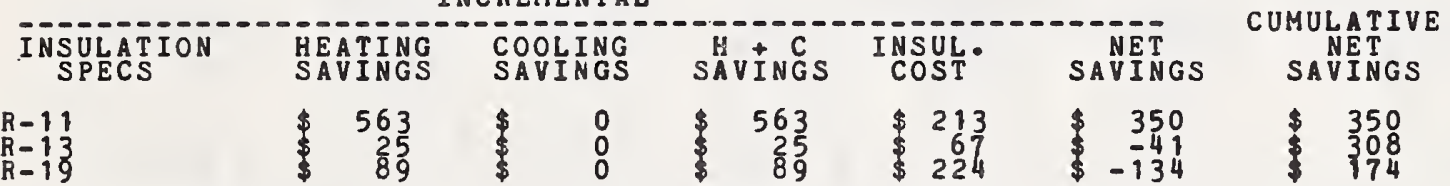

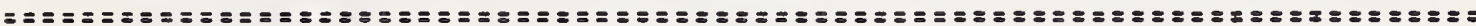

OIL FURNACE
ELECTRIC AIR COND. $\$$ \$490/GALLON $:$ PWF $=25.931$

INCREMENTAL

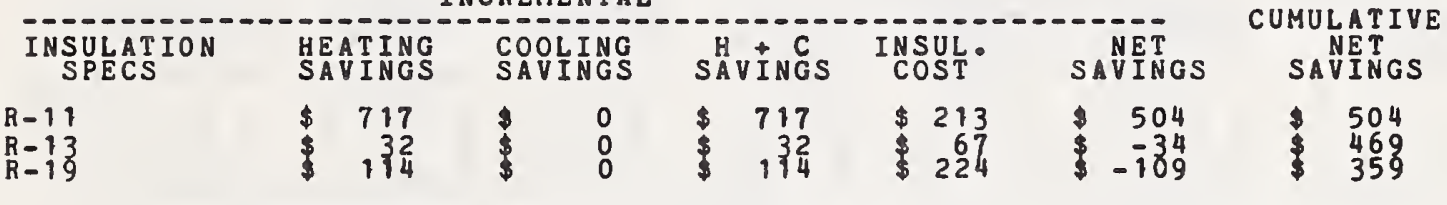

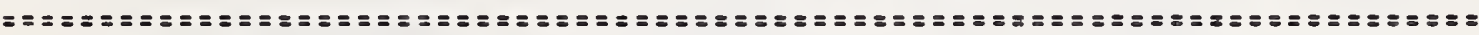

ELECTRIC FURNACE $\$: 035 /$ KHH $: P W F=18.480$

INCREMENTAL

INSULATION HEATING COOLING HAtC INSUL

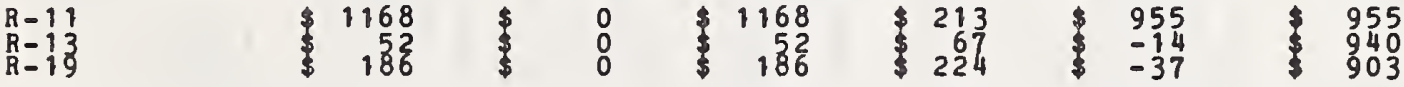

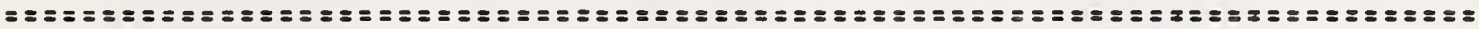
HEAT PUMP
ELECTRIC AIR COND.

INCREMENTAL

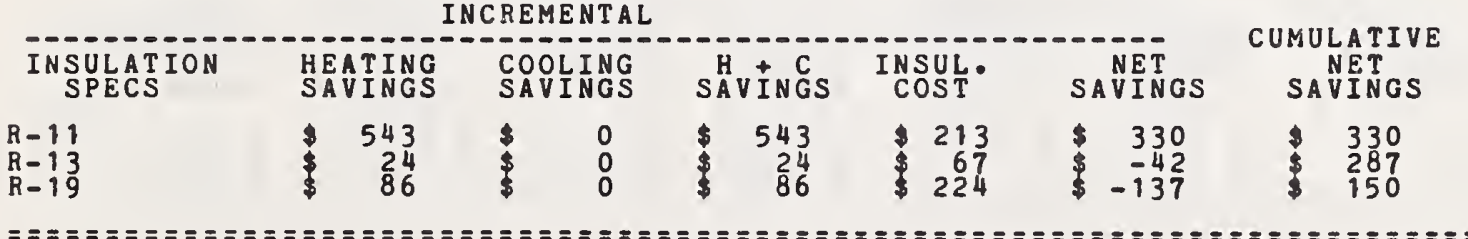


HUD5

INDIANAPOLIS INDIANA 200 SO FT HOUSE WOOD FRAME WALL COOLING LOADS BASED ON NO COOLING SAVINGS

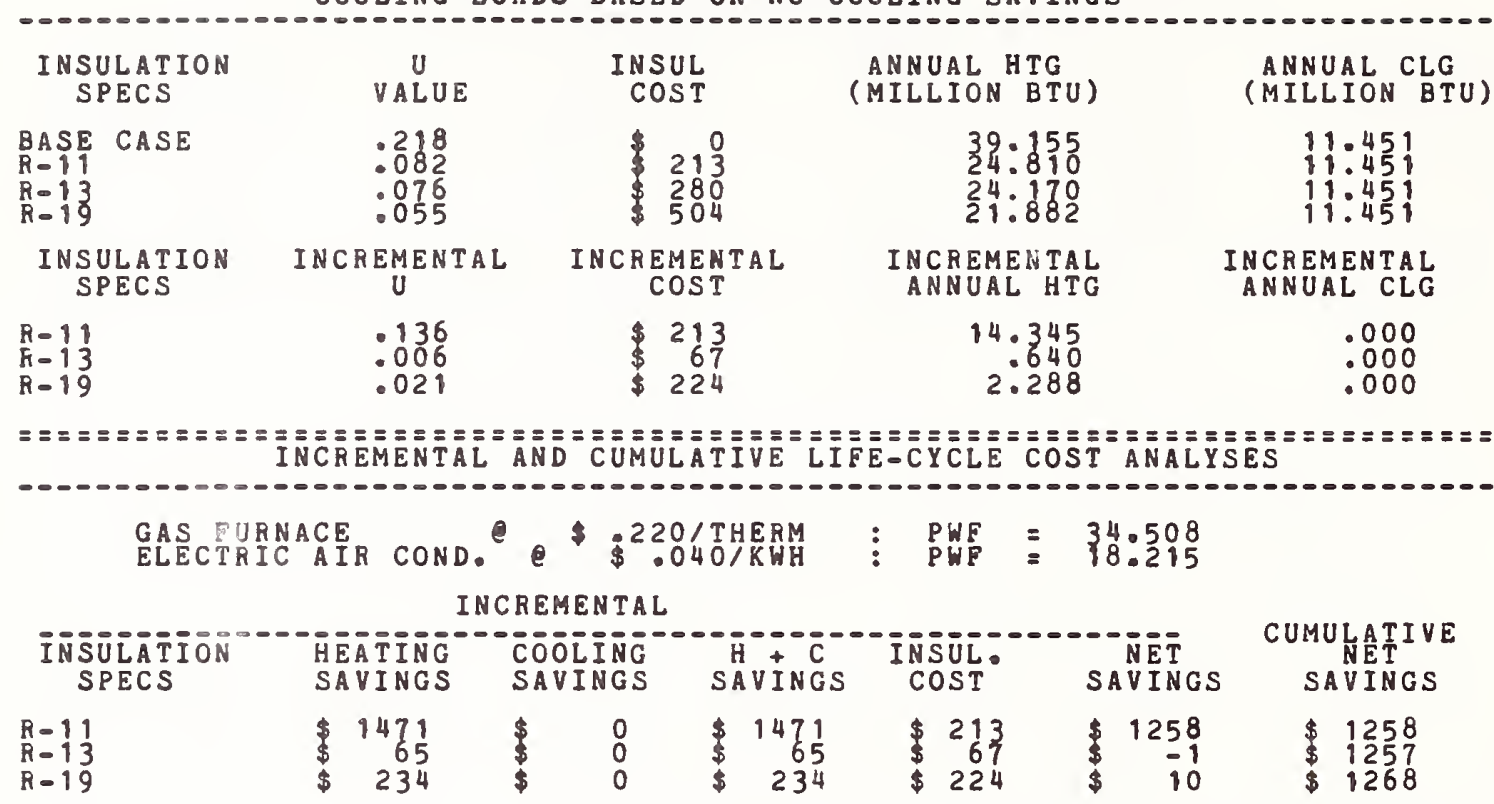

OIL FURNACE
ELECTRICAIR COND. e $e^{\$ 470 / G A L L O N}:$ PWF $=24.960$

INCREMENTAL

\begin{tabular}{|c|c|c|c|c|c|c|}
\hline $\begin{array}{l}\text { NSULATION } \\
\text { SPECS }\end{array}$ & $\begin{array}{l}\text { HEATING } \\
\text { SAVINGS }\end{array}$ & $\begin{array}{l}\text { COOLING } \\
\text { SAVINGS }\end{array}$ & $\begin{array}{l}\text { HUVINGS } \\
\text { SAVI }\end{array}$ & $\begin{array}{l}\text { INSUL. } \\
\text { COST }\end{array}$ & $\begin{array}{l}\text { NET } \\
\text { SAVINGS }\end{array}$ & SAVINGS \\
\hline $\begin{array}{r}-11 \\
-13 \\
-19\end{array}$ & $\begin{array}{r}1540 \\
\$ \quad 28 \\
\$ \quad 245\end{array}$ & $\$$ & $\begin{array}{r}1540 \\
\quad 68 \\
245\end{array}$ & $\begin{array}{r}213 \\
67 \\
224\end{array}$ & $\begin{array}{r}1327 \\
1 \\
21\end{array}$ & $\begin{array}{l}1327 \\
\$ 1329 \\
\$ 1350\end{array}$ \\
\hline
\end{tabular}

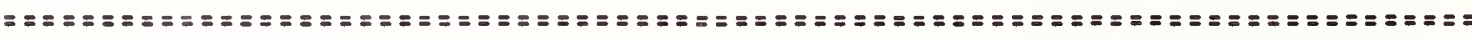

ELECTFIC FURNACE e e $\$ .040 / K W H$
ELECTRIC AIR COND. e $\$$ PWF $=18.215$

INCREMENTAL

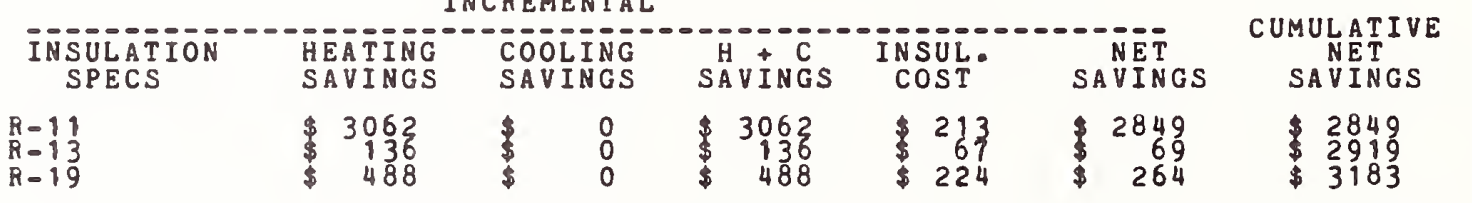

HEAT PUMP
ELECTRIC AIR COND。 e $\$ 040 / K W H$

INCREMENTAL

\begin{tabular}{|c|c|c|c|c|c|c|}
\hline $\begin{array}{l}\text { INSULATION } \\
\text { SPECS }\end{array}$ & $\begin{array}{l}\text { HEATING } \\
\text { SAVINGS }\end{array}$ & $\begin{array}{l}\text { COLING } \\
\text { SAVINGS }\end{array}$ & SAVINGS & $\begin{array}{l}\text { INSUL. } \\
\text { COST }\end{array}$ & $\begin{array}{l}\text { NET } \\
\text { SAVINGS }\end{array}$ & $\begin{array}{r}\text { CUMULATIV } \\
\text { NET } \\
\text { SAVINGS }\end{array}$ \\
\hline $\begin{aligned}-11 \\
=13\end{aligned}$ & $\begin{array}{r}1655 \\
\quad \$ 7 \\
264\end{array}$ & $\$$ & $\begin{array}{r}1655 \\
\$ \quad 73 \\
264\end{array}$ & $\begin{array}{r}213 \\
\$ 27 \\
224\end{array}$ & $\begin{array}{r}1442 \\
6 \\
40\end{array}$ & $\begin{array}{l}1442 \\
\$ 1449 \\
\$ 1489\end{array}$ \\
\hline
\end{tabular}


HUD5 JACKSONVILLE FLORIDA
COOLING LOADS BASED ON NO HOUSE COOLING SAVINGS

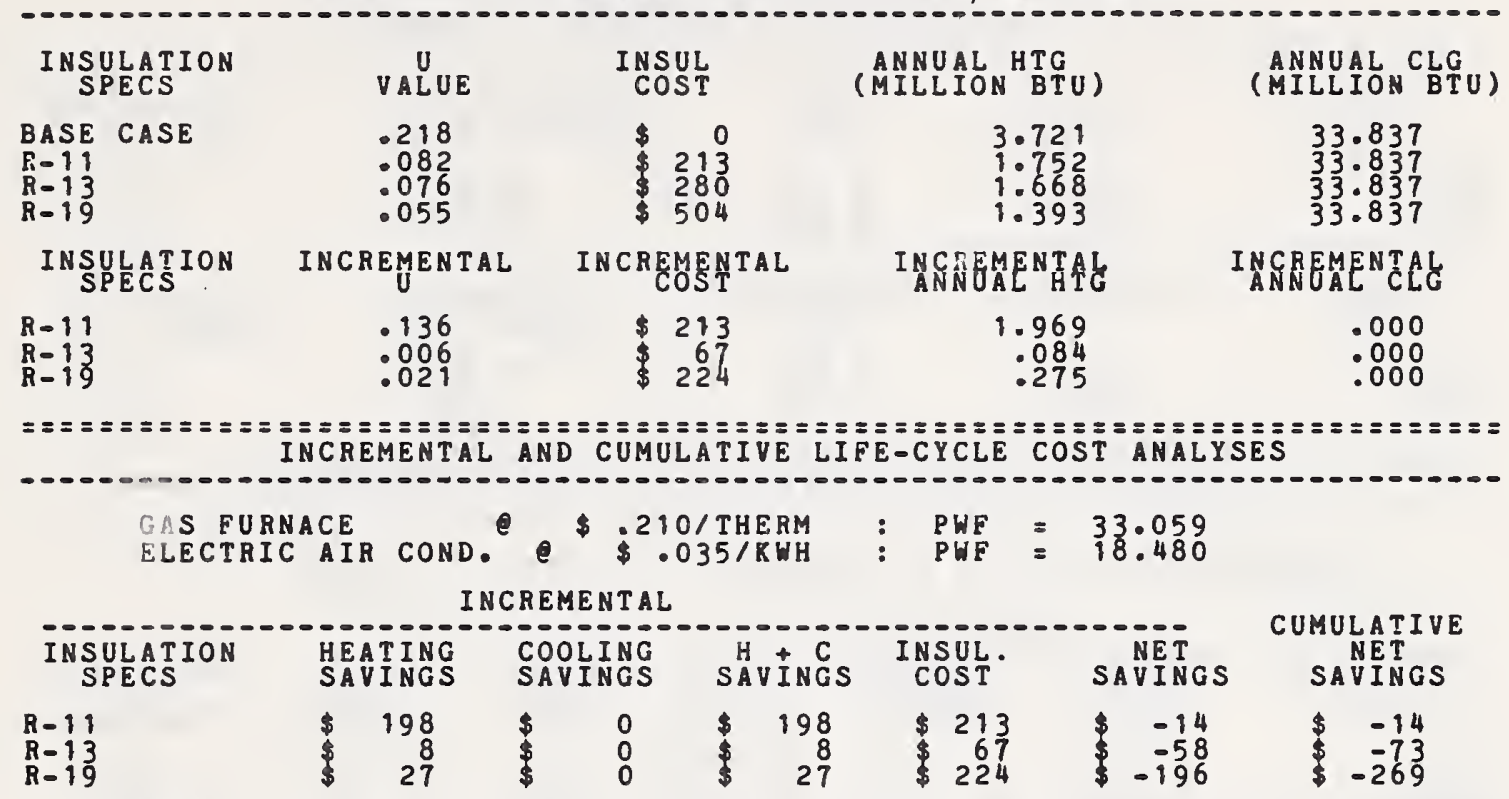

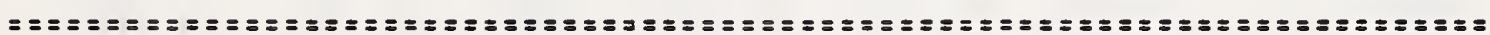

OIL FURNACE
ELECTRICAIR COND. $e^{\$ 490 / G A L L O N}: \begin{aligned} & \text { PWF } \\ & \text { PWF }\end{aligned}=25.935 \%$ KHH

INCREMENTAL

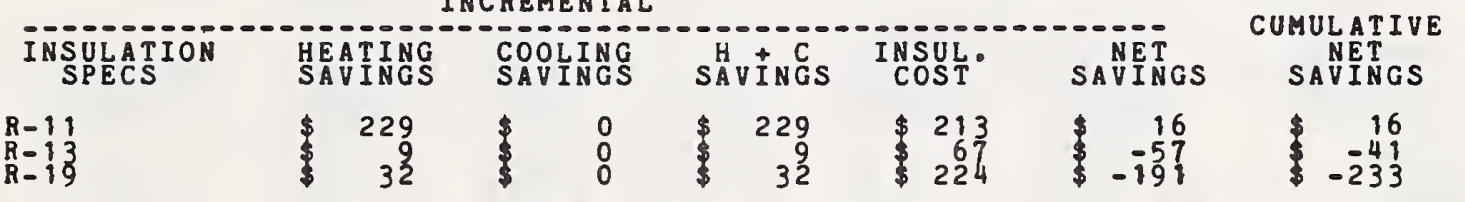

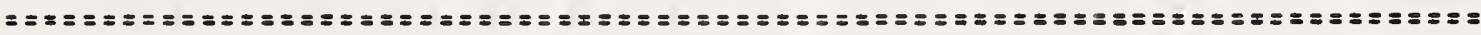

ELECTRIC FURNACE $\$$ PWF $=18.480$

ELECTRIC AIR COND. $\quad \$ .035 / K W H:$ PWF $=18.480$

\begin{tabular}{|c|c|c|c|c|c|c|c|c|c|c|}
\hline \multirow{2}{*}{$\begin{array}{l}\text { INSULATION } \\
\text { SPECS } \\
R-11 \\
R-13 \\
R-19\end{array}$} & \multicolumn{4}{|c|}{ INCREMENTAL } & \multicolumn{2}{|c|}{ SÄVINGS } & $\begin{array}{l}\text { INSUL. } \\
\text { COST }\end{array}$ & SAVETES & \multicolumn{2}{|c|}{$\begin{array}{c}\text { CUMULATIVE } \\
\text { NETETINGS }\end{array}$} \\
\hline & $\$$ & $\begin{array}{r}373 \\
15 \\
52\end{array}$ & $\$$ & $\begin{array}{l}0 \\
0 \\
0\end{array}$ & $\$$ & $\begin{array}{r}373 \\
15 \\
52\end{array}$ & $\begin{array}{r}213 \\
\$ \quad 67 \\
\$ 224\end{array}$ & $\begin{array}{r}160 \\
-51 \\
-171\end{array}$ & $\$$ & $\begin{array}{l}160 \\
109 \\
-62\end{array}$ \\
\hline
\end{tabular}

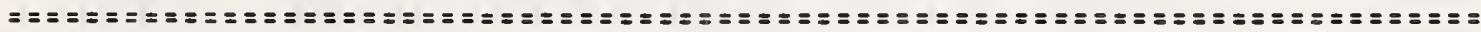
HEAT PUMP
ELECTRIC AIR COND. e $\$ .035 / K W H$
$\$ P W H F$

INCREMENTAL

\begin{tabular}{|c|c|c|c|c|c|c|}
\hline $\begin{array}{l}\text { INSULATION } \\
\text { SPECS }\end{array}$ & $\begin{array}{l}\text { HEATING } \\
\text { SAVINGS }\end{array}$ & $\begin{array}{l}\text { COOLING } \\
\text { SAVINGS }\end{array}$ & SÄVINGS & INSUL. & SAVITES & $\begin{array}{c}\text { COMULATIVE } \\
\text { NETINGS }\end{array}$ \\
\hline $\begin{array}{l}R-11 \\
R-13 \\
R-19\end{array}$ & $\begin{array}{r}162 \\
\quad 22 \\
\$ \quad 22\end{array}$ & $\$$ & $\begin{array}{r}162 \\
\$ \quad 22\end{array}$ & $\begin{array}{r}213 \\
\quad 67 \\
224\end{array}$ & $\begin{array}{r}-50 \\
-60 \\
-201\end{array}$ & $\begin{array}{r}-50 \\
-110 \\
-312\end{array}$ \\
\hline
\end{tabular}


HUD5

MADISON WISCONSIN WOOD FRAME WALL COOLING LOADS BASED ON NO COOLING SAVINGS

\begin{tabular}{|c|c|c|c|c|}
\hline $\begin{array}{l}\text { INSULAT ION } \\
\text { SPECS }\end{array}$ & $\begin{array}{l}\text { UALUE } \\
\text { VALU }\end{array}$ & $\begin{array}{l}\text { INSUL } \\
\text { COST }\end{array}$ & $\begin{array}{l}\text { ANNUAL HTG } \\
\text { (MILLION BTU) }\end{array}$ & $\begin{array}{l}\text { ANNUAL CLG } \\
\text { (MILLION BTU) }\end{array}$ \\
\hline $\begin{array}{l}\text { BASE CASE } \\
R=19 \\
B=13 \\
R=19\end{array}$ & $\begin{array}{r}.218 \\
.082 \\
.076 \\
.055\end{array}$ & $\begin{array}{rr} & 0 \\
& 213 \\
& 280 \\
& 2804\end{array}$ & $\begin{array}{l}46.100 \\
28: 236 \\
27: 440 \\
24.604\end{array}$ & $\begin{array}{l}6.381 \\
6: 381 \\
6: 381 \\
6: 381\end{array}$ \\
\hline $\begin{array}{l}\text { INSULATION } \\
\text { SPECS }\end{array}$ & INCREME & $\begin{array}{c}\text { INCREMENTAL } \\
\text { COST }\end{array}$ & $\begin{array}{l}\text { INCREMENTAL } \\
\text { ANNUAL HTG }\end{array}$ & $\begin{array}{l}\text { INCREMENTAL } \\
\text { ANNUAL CLG }\end{array}$ \\
\hline $\begin{array}{l}R=11 \\
R=13 \\
R=19\end{array}$ & $\begin{array}{l}.136 \\
.006 \\
.021\end{array}$ & $\begin{array}{r}213 \\
627 \\
224\end{array}$ & $\begin{array}{r}17.863 \\
2.796 \\
37\end{array}$ & $\begin{array}{l}.000 \\
.000 \\
.000\end{array}$ \\
\hline
\end{tabular}

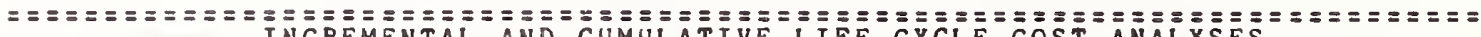
INCREMENTAL AND CUMULATIVE LIFE-CYCLE COST ANALYSES

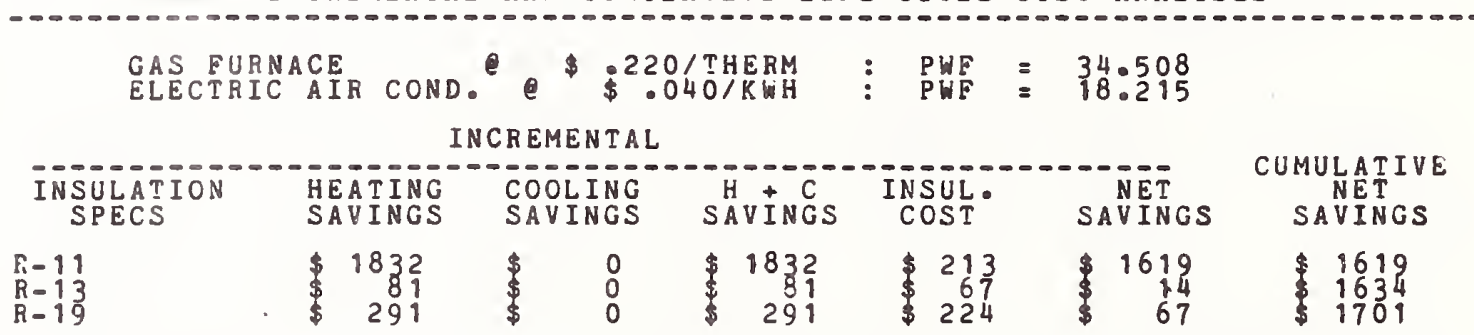

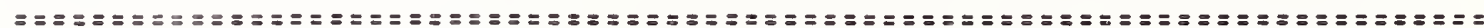
CILEURNACE

INCREMENTAL

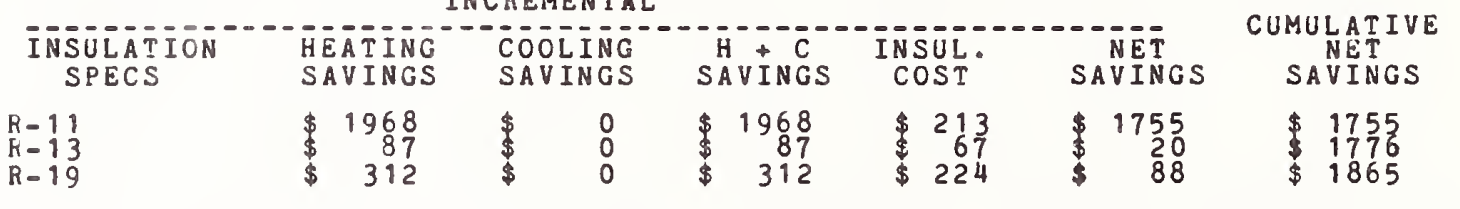

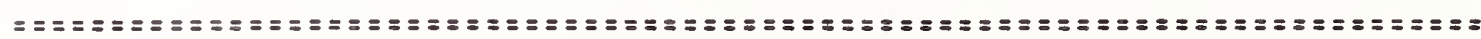

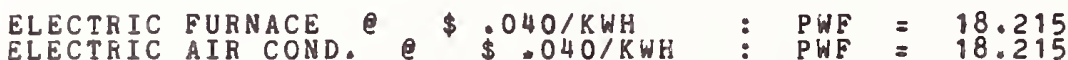

INCREMENTAL

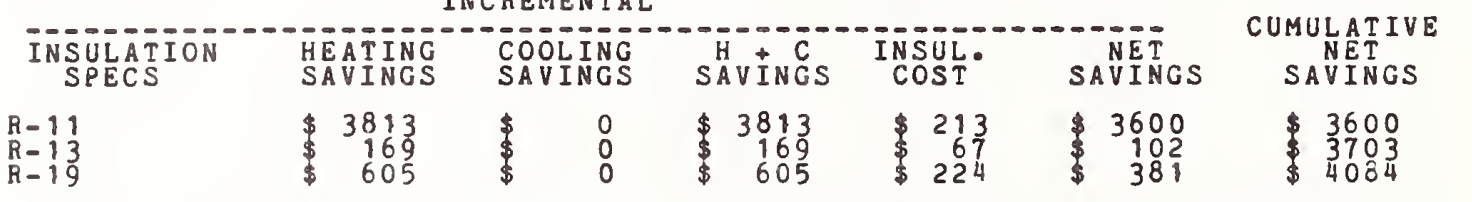

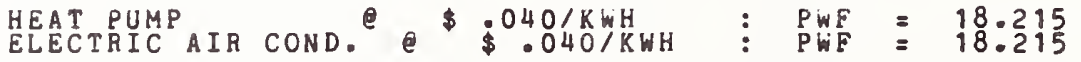

INCREMENTAL

\begin{tabular}{|c|c|c|c|c|c|c|}
\hline $\begin{array}{l}\text { INSULATION } \\
\text { SPECS }\end{array}$ & $\begin{array}{l}\text { HEATING } \\
\text { SAVINGS }\end{array}$ & $\begin{array}{l}\text { COOLING } \\
\text { SAVINGS }\end{array}$ & SAVINGS & $\begin{array}{l}\text { INSUL. } \\
\text { COST }\end{array}$ & $\begin{array}{l}\text { NET } \\
\text { SAVINGS }\end{array}$ & $\begin{array}{l}\text { UMULATIVE } \\
\text { NET } \\
\text { SAVINGS }\end{array}$ \\
\hline $\begin{array}{r}-11 \\
-13 \\
-19\end{array}$ & $\begin{array}{r}2460 \\
109 \\
\$ 390\end{array}$ & $\$$ & $\begin{array}{r}2460 \\
109 \\
\$ 390\end{array}$ & $\begin{array}{r}213 \\
\$ 224\end{array}$ & $\begin{array}{r}2247 \\
\$ 42 \\
\$ 166\end{array}$ & $\begin{array}{l}2247 \\
\$ 2289 \\
\$ 2456\end{array}$ \\
\hline
\end{tabular}

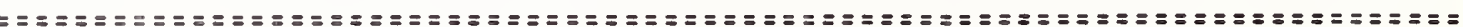


HUD5 PHOENIX ARIZONA 1200 SOFT HOUSE WOOD ERAME WALL

\begin{tabular}{|c|c|c|c|c|}
\hline $\begin{array}{l}\text { INSULATION } \\
\text { SPECS }\end{array}$ & VALUE & $\begin{array}{l}\text { INSUL } \\
\text { COST }\end{array}$ & $\begin{array}{l}\text { ANNUAL HTG } \\
\text { (MILLION }\end{array}$ & (ANNUAL CLG \\
\hline $\begin{array}{l}\text { BASE CASE } \\
R=11 \\
R=13 \\
R=19\end{array}$ & $\begin{array}{r}.218 \\
.082 \\
.076 \\
.055\end{array}$ & $\begin{array}{l}\$ \\
213 \\
283 \\
2804 \\
\$ 504\end{array}$ & $\begin{array}{l}4.479 \\
2.079 \\
1.975 \\
1.625\end{array}$ & $\begin{array}{l}43.617 \\
43.617 \\
43.617 \\
43.617\end{array}$ \\
\hline $\begin{array}{l}\text { INSULATION } \\
\text { SPECS }\end{array}$ & INCREM & $\begin{array}{c}\text { INCREMENTAL } \\
\text { COST }\end{array}$ & $\begin{array}{l}\text { INCREMENTAL } \\
\text { ANNUAL HTG }\end{array}$ & $\begin{array}{l}\text { INCKEMENTAL } \\
\text { ANNUAL CLG }\end{array}$ \\
\hline $\begin{array}{l}R-11 \\
R=13 \\
R-19\end{array}$ & $\begin{array}{r}136 \\
: 006 \\
.021\end{array}$ & $\begin{array}{rr}\$ 213 \\
\$ 27 \\
\$ 224\end{array}$ & $\begin{array}{r}2.400 \\
.104 \\
.350\end{array}$ & $\begin{array}{l}.000 \\
.000 \\
.000\end{array}$ \\
\hline
\end{tabular}

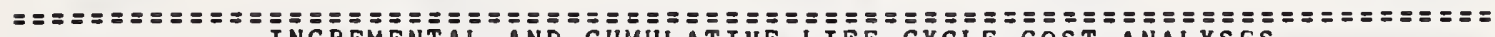
INCREMENTAL AND CUMULATIVE LIFE-CYCLE COST ANALYSES

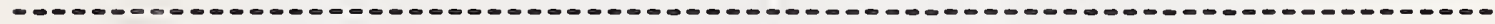

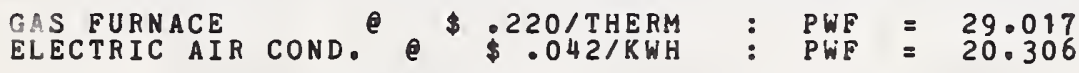

INCREMENTAL

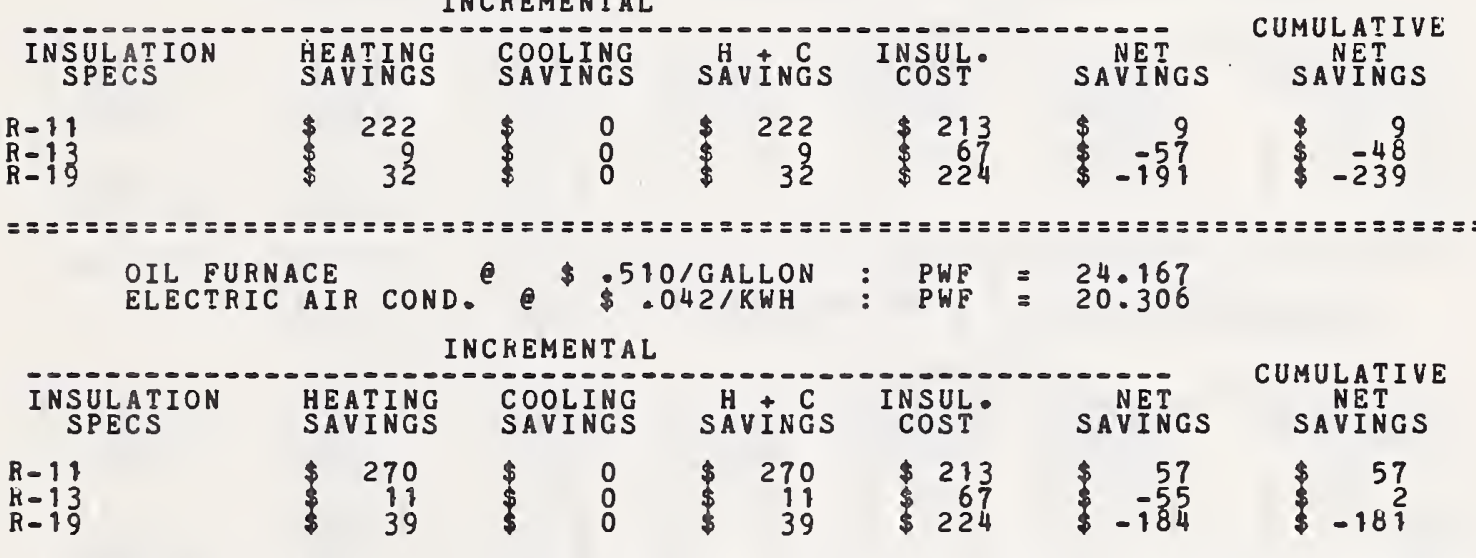

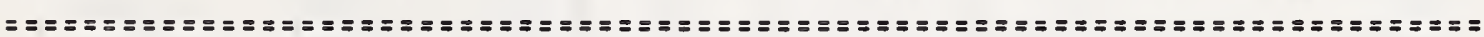

ELECTRIC FURNACE
ELECTRIC AIR COND. $\$ \$ 042 / K W H$
PWF $=20.306$

INCREMENTAL

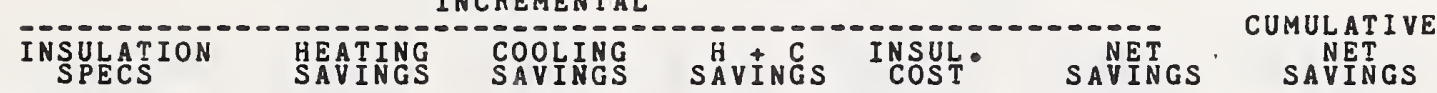

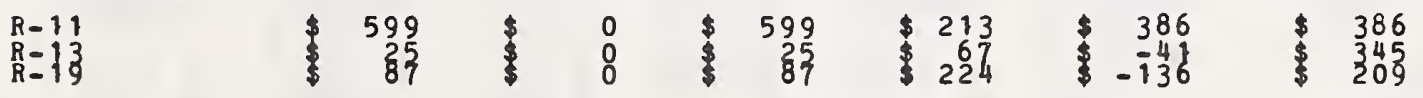

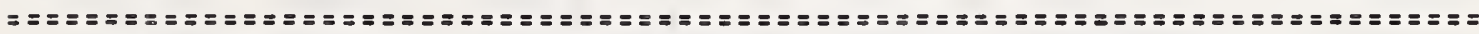

HEAT PUMP $\quad .042 / K W H \quad P W F=20.306$

ELECTRIC AIR COND. $\$ .042 / \mathrm{KWH}:$ PWF $=20.306$

INCREMENTAL

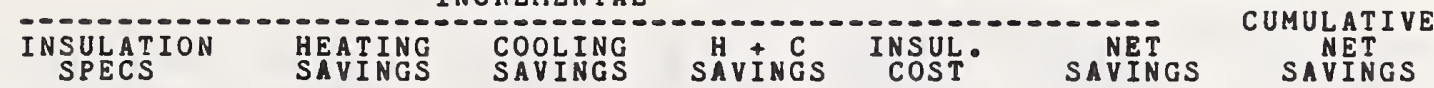

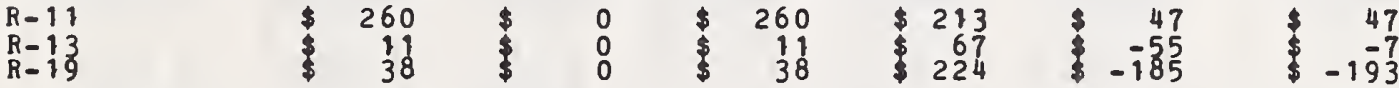

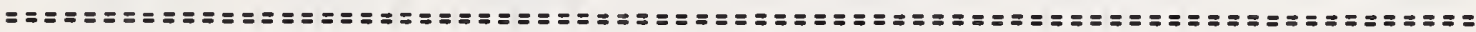


HUD5

TAMPA FLORIDA WOOD FRAME WALL COOLING LOADS BASED ON NO COOLING SAVINGS

\begin{tabular}{|c|c|c|c|c|}
\hline $\begin{array}{l}\text { INSULATION } \\
\text { SPECS }\end{array}$ & VALUE & $\begin{array}{l}\text { INSUL } \\
\text { COST }\end{array}$ & $\begin{array}{l}\text { ANNUAL HTG } \\
\text { (MILLION BTU) }\end{array}$ & $\begin{array}{l}\text { ANNUAL CLG } \\
\text { (MILLION BTU) }\end{array}$ \\
\hline $\begin{array}{l}B A S E \text { CASE } \\
R=11 \\
R=13 \\
R-19\end{array}$ & $\begin{array}{l}.218 \\
.082 \\
.076 \\
.055\end{array}$ & $\begin{array}{r}\$ 210 \\
\$ 280 \\
\$ 504\end{array}$ & $\begin{array}{r}.882 \\
: 349 \\
: 326 \\
.244\end{array}$ & $\begin{array}{l}39.031 \\
39.031 \\
39.031 \\
39.031\end{array}$ \\
\hline $\begin{array}{l}\text { INSULATION } \\
\text { SPECS }\end{array}$ & INCREMENTAL & $\begin{array}{c}\text { INCREMENTAL } \\
\text { COST }\end{array}$ & $\begin{array}{l}\text { INCREMENTAL } \\
\text { ANNUAL HTG }\end{array}$ & $\begin{array}{l}\text { INCREMENTAL } \\
\text { AN NUAL CLG }\end{array}$ \\
\hline $\begin{array}{l}R=11 \\
R=13 \\
R=19\end{array}$ & $\begin{array}{l}.136 \\
.006 \\
.021\end{array}$ & $\begin{array}{r}213 \\
\$ 224 \\
\$ 224\end{array}$ & $\begin{array}{l}.532 \\
.023 \\
.082\end{array}$ & $\begin{array}{l}.000 \\
.000 \\
.000\end{array}$ \\
\hline
\end{tabular}

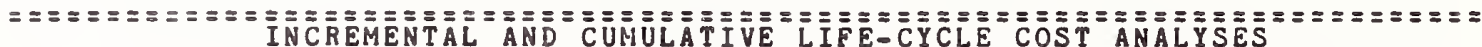

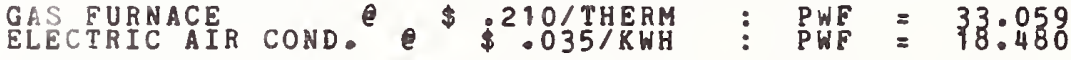

INCREMENTAL

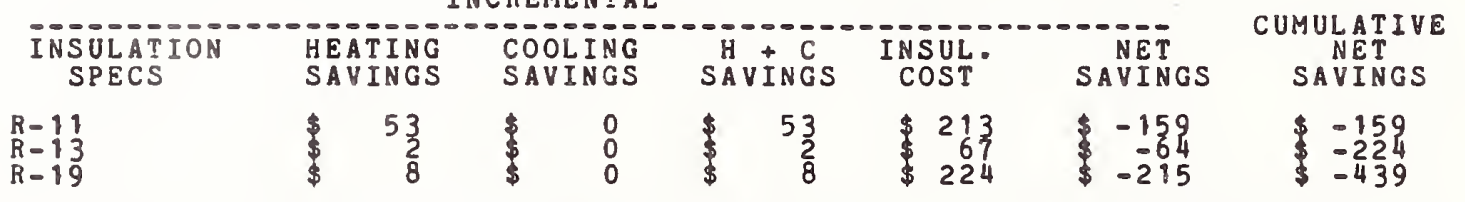

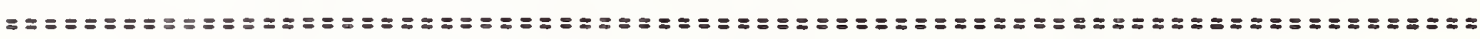

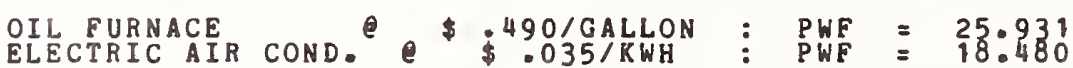

IN C REMENTAL

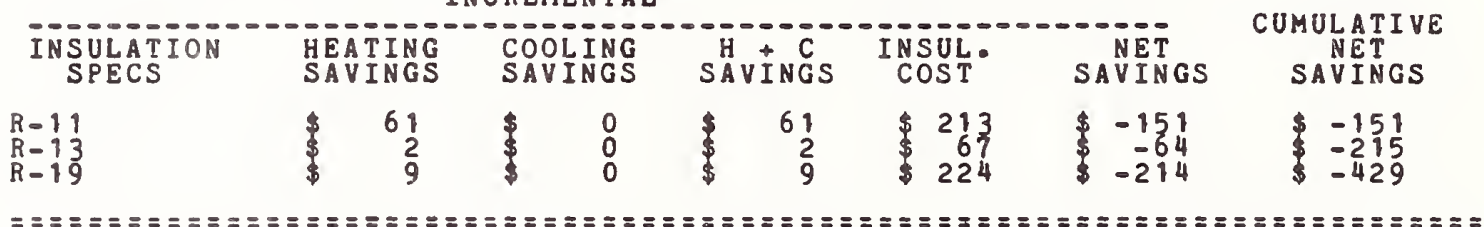

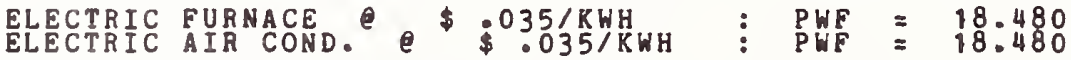

INCREMENTAL

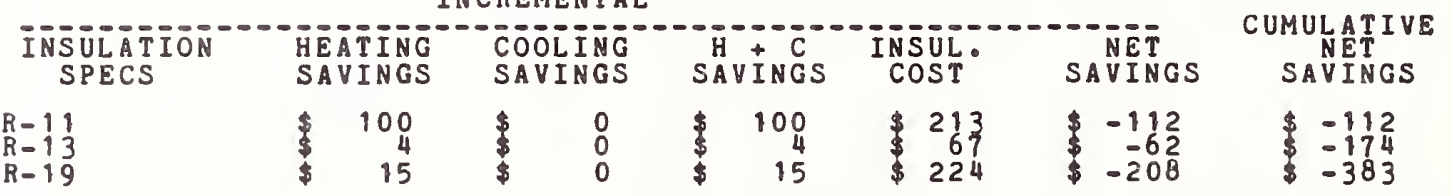

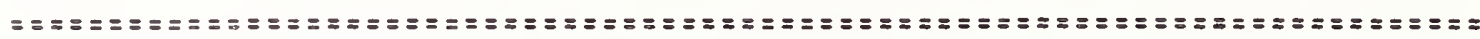
HEAT PUMP
ELECTRIC AIR COND. e $\$ \$ 035 / K W H$

I N C REMEN TAL

\begin{tabular}{|c|c|c|c|c|c|c|}
\hline $\begin{array}{l}\text { INSULATION } \\
\text { SPECS }\end{array}$ & $\begin{array}{l}\text { HEAT ING } \\
\text { SAVINGS }\end{array}$ & $\begin{array}{l}\text { COOLING } \\
\text { SAVINGS }\end{array}$ & SÄVINGS & $\begin{array}{l}\text { INSUL. } \\
\text { COST }\end{array}$ & SAVINGS & $\begin{array}{l}\text { CUMULATIVE } \\
\text { NET } \\
\text { SAVINGS }\end{array}$ \\
\hline 13 & $\begin{array}{r}43 \\
6 \\
6\end{array}$ & $\$$ & $\begin{array}{r}43 \\
6\end{array}$ & $\begin{array}{r}213 \\
67 \\
224\end{array}$ & $\begin{array}{r}-169 \\
\$-65 \\
-217\end{array}$ & $\begin{array}{l}-169 \\
-234 \\
-451\end{array}$ \\
\hline
\end{tabular}

= = = = = 
HUD5 WASHINGTON D.C I2OO SQFT HOUSE WOOD FRAME WALL
COOLING LOADS BASED ON NO COOLING SAVINGS

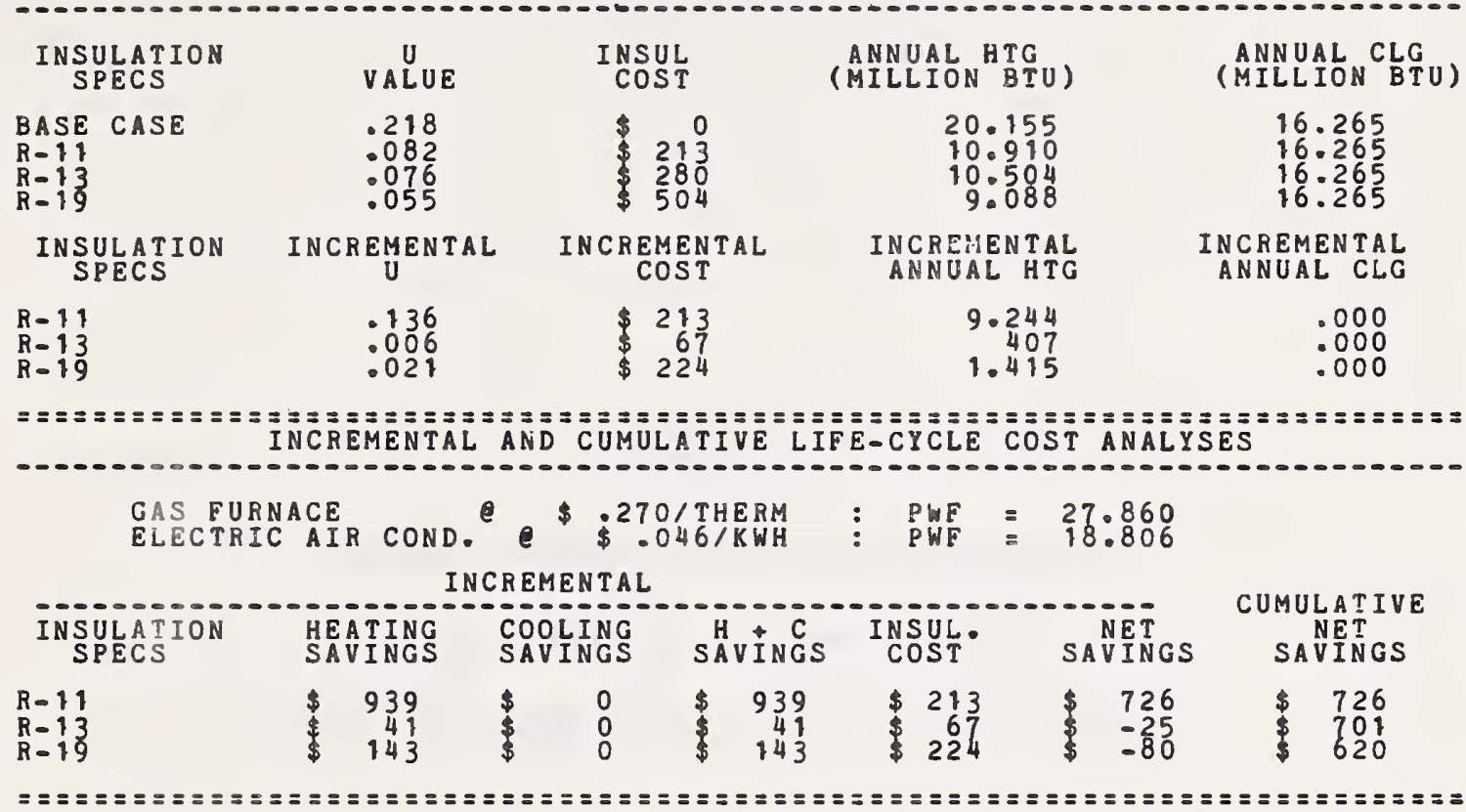

OIL FURNACE
ELECTRICAIR COND. e $e^{\$ 500 / G A L L O N}:$ PWF $=25.324$
PWF $=18.806 / K W H$

I NCREMENTAL

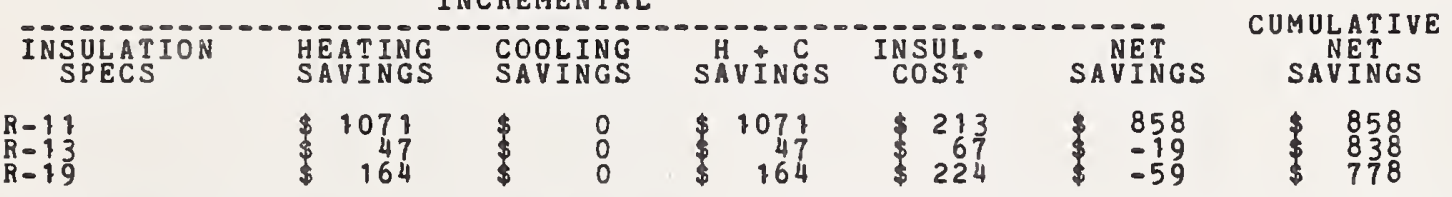

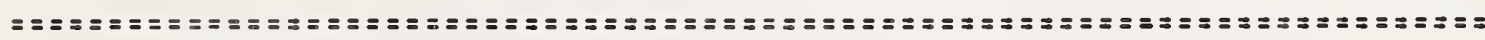
ELECTRIC FURNACE e $\$ .046 / K W H$
ELECTRIC AIR COND. $\$$ PWF $=18.806$ INCREMENTAL

\begin{tabular}{|c|c|c|c|c|c|c|}
\hline $\begin{array}{l}\text { INSULAT ION } \\
\text { SPECS }\end{array}$ & $\begin{array}{l}\text { HEATING } \\
\text { SAVINGS }\end{array}$ & $\begin{array}{l}\text { COOLING } \\
\text { SAVINGS }\end{array}$ & SAVINGS & $\begin{array}{l}\text { INSUL. } \\
\text { COST }\end{array}$ & $\begin{array}{l}\text { NET } \\
\text { SAVINGS }\end{array}$ & $\begin{array}{l}\text { CUMULATIVE } \\
\text { NET } \\
\text { SAVINGS }\end{array}$ \\
\hline $\begin{array}{l}-11 \\
-13 \\
-19\end{array}$ & $\begin{array}{r}2343 \\
103 \\
\$ 358\end{array}$ & $\$$ & $\begin{array}{r}2343 \\
103 \\
\$ 358\end{array}$ & $\begin{array}{r}213 \\
67 \\
224\end{array}$ & $\begin{array}{r}2130 \\
36 \\
134\end{array}$ & $\begin{array}{l}2130 \\
\$ 2166 \\
\$ 2300\end{array}$ \\
\hline
\end{tabular}

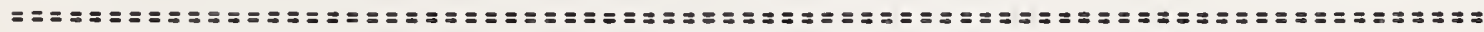
HEAT PUMP
ELECTRIC AIR COND. e $\$ .046 / K W H$
$\$ 046 / K W H$ INCREMENTAL

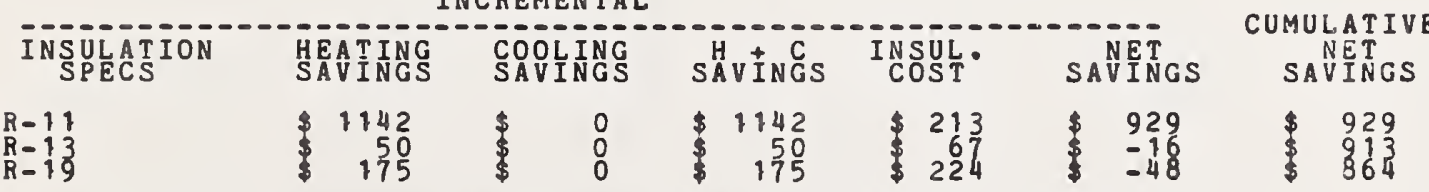

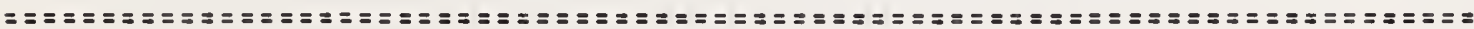





\begin{abstract}
APPENDIX B
Computer Printouts of Economic Analyses

for Tampa, Phoenix and Jacksonville --

for Cooling Savings Based on Windows Closed
\end{abstract}


HUD 1

JACRSONVILLE ELORIDA 1200 SQ FT HOUSE 8 IN BLOCK (OPEN CORE) COOLING LOADS BASED ON WINDOWS CLOSED

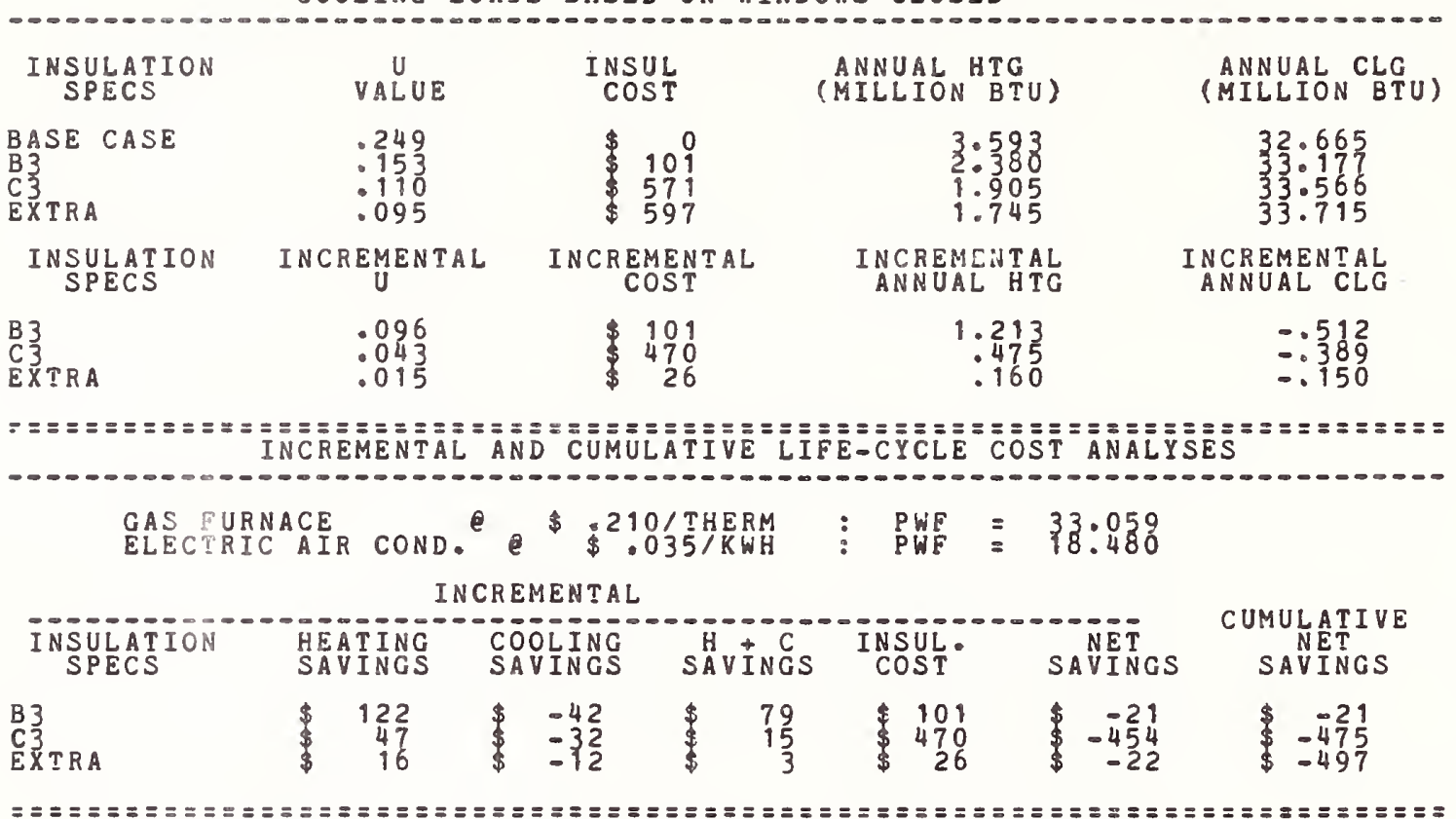

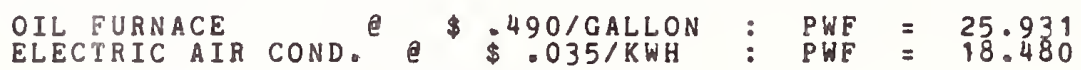

INCREMENTAL

\begin{tabular}{|c|c|c|c|c|c|c|}
\hline $\begin{array}{l}\text { INSULAT ION } \\
\text { SPECS }\end{array}$ & $\begin{array}{l}\text { HEATING } \\
\text { SAVINGS }\end{array}$ & $\begin{array}{l}\text { COOLING } \\
\text { SAVINGS }\end{array}$ & SAVI C & $\begin{array}{l}\text { INSUL. } \\
\text { COST }\end{array}$ & $\begin{array}{l}\text { NET } \\
\text { SAVINGS }\end{array}$ & $\begin{array}{c}\text { CUMULATIVE } \\
\text { NET } \\
\text { SAVINGS }\end{array}$ \\
\hline TRA & $\begin{array}{r}\$ 141 \\
\$ \quad 55 \\
\$ \quad 18\end{array}$ & $\begin{array}{l}-42 \\
\$-32 \\
-12\end{array}$ & $\begin{array}{r}98 \\
23 \\
6\end{array}$ & $\begin{array}{r}101 \\
\$ 470 \\
\$ \quad 26\end{array}$ & $\begin{array}{r}\$-2 \\
\$-44 \\
-19\end{array}$ & $\begin{array}{l}\$-2 \\
-448 \\
-468\end{array}$ \\
\hline
\end{tabular}

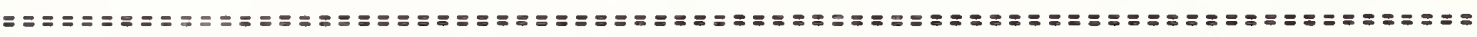

ELECTRIC FURNACE e $\$ 035 / K H H$
ELECTRIC AIR COND. $\$ \$ P W F=18.480$ INCREMENTAL

\begin{tabular}{|c|c|c|c|c|c|c|c|c|}
\hline $\begin{array}{l}\text { INSULATION } \\
\text { SPECS }\end{array}$ & & $\begin{array}{l}\text { TING } \\
\text { INGS }\end{array}$ & & $\begin{array}{l}\text { L ING } \\
\text { INGS }\end{array}$ & SAVINC & $\begin{array}{l}\text { INSUL. } \\
\text { COST }\end{array}$ & $\begin{array}{l}\text { NET } \\
\text { SAVINGS }\end{array}$ & SAVINGS \\
\hline TRA & $\$$ & $\begin{array}{r}229 \\
90 \\
30\end{array}$ & $\$$ & $\begin{array}{l}-42 \\
-32 \\
-12\end{array}$ & $\begin{array}{r}187 \\
\quad 57 \\
\quad 17\end{array}$ & $\begin{array}{r}101 \\
\$ \quad 470 \\
\$ \quad 26\end{array}$ & $\begin{array}{r}86 \\
\$-412 \\
\$-8\end{array}$ & $\begin{array}{r}86 \\
\$-325 \\
-333\end{array}$ \\
\hline
\end{tabular}

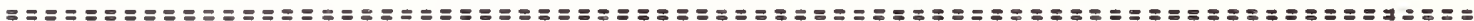

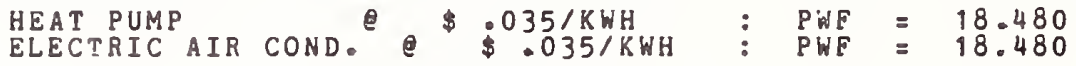

INCREMENTAL

\begin{tabular}{|c|c|c|c|c|c|c|}
\hline $\begin{array}{l}\text { NSULATION } \\
\text { SPECS }\end{array}$ & $\begin{array}{l}\text { HEAT ING } \\
\text { SAVINGS }\end{array}$ & $\begin{array}{l}\text { COOLING } \\
\text { SAVINGS }\end{array}$ & SAVINGS & $\begin{array}{l}\text { INSUL. } \\
\text { COST }\end{array}$ & SAVINGS & $\begin{array}{l}\text { NET } \\
\text { SAVINGS }\end{array}$ \\
\hline R A & $\begin{array}{l}99 \\
39 \\
13\end{array}$ & $\begin{array}{l}\$-44 \\
\$-33 \\
\$ \quad-12\end{array}$ & $\begin{array}{r}55 \\
5 \\
0\end{array}$ & $\begin{array}{r}101 \\
470 \\
\$ 26\end{array}$ & $\begin{array}{r}\$-45 \\
\$-464 \\
\$\end{array}$ & $\begin{array}{l}-45 \\
\$-509 \\
-535\end{array}$ \\
\hline
\end{tabular}

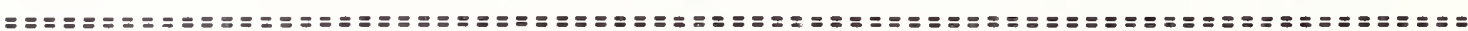


HUD 1 PHOENIX ARIZONA 1200 SQ FT HOUSE 8 IN BLOCK (OPEN CORE) COOLING LOADS BASED ON WINDOWS CLOSED

\begin{tabular}{|c|c|c|c|c|}
\hline $\begin{array}{l}\text { INSULATION } \\
\text { SPECS }\end{array}$ & $\stackrel{U}{\text { VALUE }}$ & $\begin{array}{l}\text { INSUL } \\
\text { COST }\end{array}$ & $\begin{array}{l}\text { ANNUAL HTG } \\
\text { (MILLION }\end{array}$ & $\begin{array}{l}\text { ANNUAL CLG } \\
\text { (MILLION BTU) }\end{array}$ \\
\hline $\begin{array}{l}\text { BASE CASE } \\
\text { B3 } \\
\text { C3 } \\
\text { EXTRA }\end{array}$ & $\begin{array}{r}.249 \\
.153 \\
.190 \\
.095\end{array}$ & $\begin{array}{r}\$ 10 \\
\$ 571 \\
\$ 597\end{array}$ & $\begin{array}{l}4.035 \\
2.685 \\
2.157 \\
1.980\end{array}$ & $\begin{array}{l}42.142 \\
40.310 \\
39.602 \\
39.364\end{array}$ \\
\hline $\begin{array}{l}\text { INSULATION } \\
\text { SPECS }\end{array}$ & INCREMENTAL & $\begin{array}{c}\text { INCREMENTAL } \\
\text { COST }\end{array}$ & $\begin{array}{l}\text { INCREMENTAL } \\
\text { AN NUAL HTG }\end{array}$ & $\begin{array}{l}\text { INCREMENTAL } \\
\text { ANNUAL CLG }\end{array}$ \\
\hline $\begin{array}{l}\text { B } 3 \\
\text { C } 3 \\
\text { EXTRA }\end{array}$ & $\begin{array}{l}.096 \\
.043 \\
.015\end{array}$ & $\begin{array}{r}101 \\
\$ \begin{array}{r}470 \\
26\end{array}\end{array}$ & $\begin{array}{r}1.349 \\
: 528 \\
: 177\end{array}$ & $\begin{array}{r}1.832 \\
.708 \\
.237\end{array}$ \\
\hline
\end{tabular}

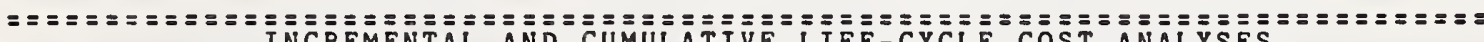
INCREMENTAL AND CUMULATIVE LIFE-CYYCLE COST ANALYSES

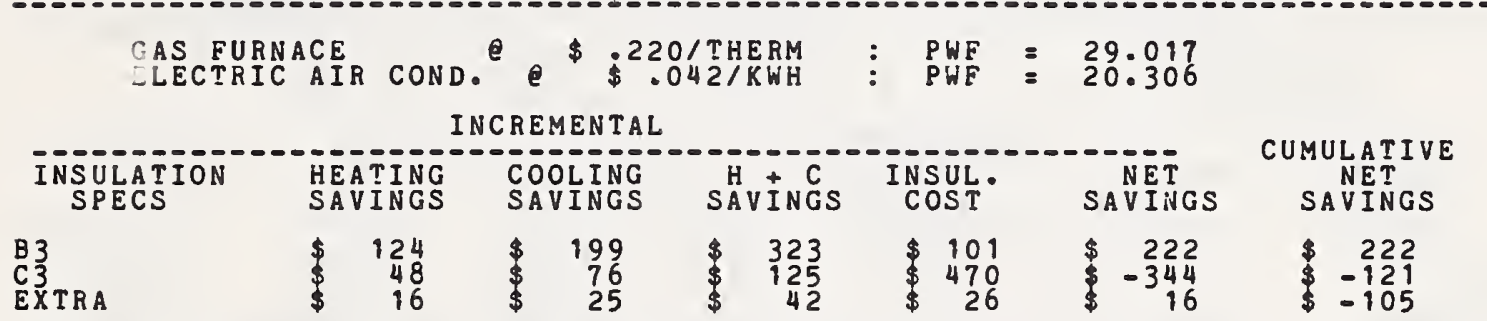

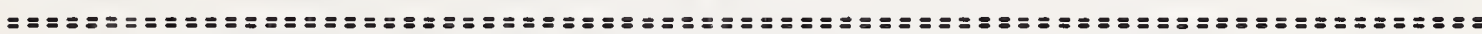

OIL FURNACE
ELECTRICAIR COND. $\$ \$$ \$10/GALLON

INCREMENTAL

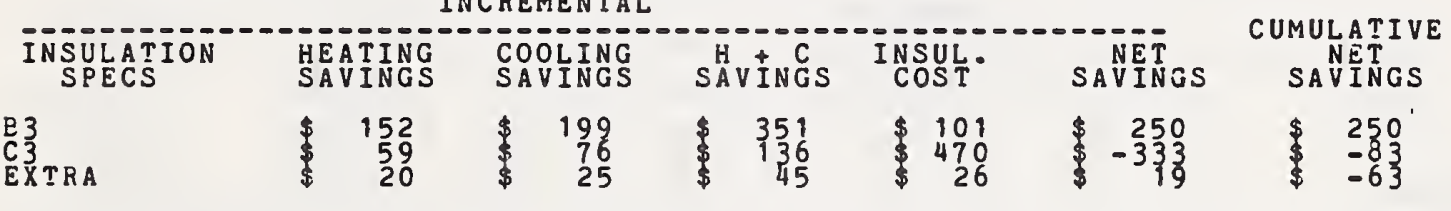

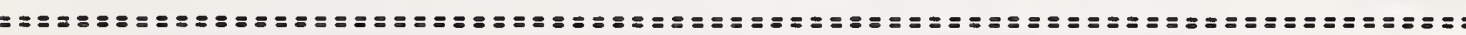

ELECTRIC FURNACE ELETRIC $\$ 042 / K W H$ P $\$$ PWF $=20.306$

INCREMENTAL

\begin{tabular}{|c|c|c|c|c|c|c|c|}
\hline $\begin{array}{l}\text { INSULATION } \\
\text { SPECS }\end{array}$ & $\begin{array}{l}\text { HEATING } \\
\text { SAVINGS }\end{array}$ & $\begin{array}{l}\text { COOLING } \\
\text { SAVINGS }\end{array}$ & $\begin{array}{l}\text { Ḧ } \\
\text { SAVINGS }\end{array}$ & $\begin{array}{l}\text { INSUL. } \\
\text { COST }\end{array}$ & SAVINGS & $\begin{array}{r}\text { CUMU } \\
\text { SA }\end{array}$ & $\begin{array}{l}\text { LATIVE } \\
\text { NET } \\
\text { VINGS }\end{array}$ \\
\hline 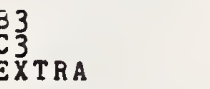 & $\begin{array}{l}\quad 337 \\
\$ \quad 137 \\
\$ \quad 44\end{array}$ & $\begin{array}{r}199 \\
76 \\
25\end{array}$ & $\begin{array}{r}536 \\
208 \\
70\end{array}$ & $\begin{array}{r}101 \\
\$ 470 \\
\$ \quad 26\end{array}$ & $\begin{array}{r}435 \\
-261 \\
\$ \\
44\end{array}$ & $\$$ & $\begin{array}{l}435 \\
174 \\
218\end{array}$ \\
\hline
\end{tabular}

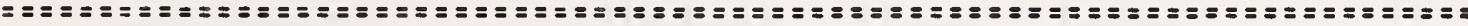

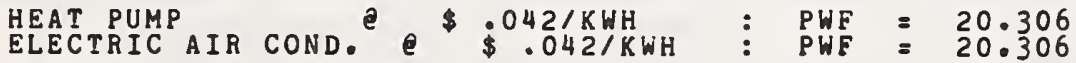

INCREMENTAL

\begin{tabular}{|c|c|c|c|c|c|c|}
\hline $\begin{array}{l}\text { INSULATION } \\
\text { SPECS }\end{array}$ & SEATING & $\begin{array}{l}\text { COOLING } \\
\text { SAVINGS }\end{array}$ & SÄVINGS & INSULT & SAVETES & $\begin{array}{c}\text { CUMULATIVE } \\
\text { SAVETTES }\end{array}$ \\
\hline $\begin{array}{l}3 \\
3 \\
X T R A\end{array}$ & $\begin{array}{r}\quad 146 \\
\$ \quad 57 \\
\$ \quad 19\end{array}$ & $\begin{array}{r}208 \\
\$ \quad 80 \\
\$ \quad 26\end{array}$ & $\begin{array}{r}354 \\
137 \\
\quad 46\end{array}$ & $\begin{array}{r}101 \\
\$ 70 \\
\$ 26\end{array}$ & $\begin{array}{r}253 \\
-332 \\
230\end{array}$ & $\begin{array}{l}253 \\
-78 \\
-58\end{array}$ \\
\hline
\end{tabular}

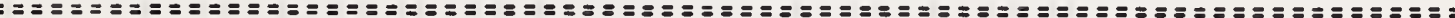


HUD 1

TAMPA ELORIDA 8 IN BLOCK (OPEN CORE) COOLING LOADS BASED ON WINDOWS CLOSED

\begin{tabular}{|c|c|c|c|c|}
\hline $\begin{array}{l}\text { INSULATION } \\
\text { SPECS }\end{array}$ & UALUE & $\begin{array}{l}\text { INSUL } \\
\text { COST }\end{array}$ & $\begin{array}{l}\text { ANNUAL HTG } \\
\text { (MILLION BTU) }\end{array}$ & $\begin{array}{l}\text { ANNUAL CLG } \\
\text { (MILLION BTU) }\end{array}$ \\
\hline $\begin{array}{l}\text { BASE CASE } \\
\text { B3 } \\
\text { C3 } \\
\text { EXTRA }\end{array}$ & $\begin{array}{r}.249 \\
.153 \\
.110 \\
.095\end{array}$ & $\begin{array}{rr}\$ & 0 \\
\$ & 101 \\
\$ & 571 \\
\$ & 597\end{array}$ & $\begin{array}{r}.850 \\
.505 \\
.383 \\
.343\end{array}$ & $\begin{array}{l}38.104 \\
38: 866 \\
39.329 \\
39.501\end{array}$ \\
\hline $\begin{array}{l}\text { INSULATION } \\
\text { SPECS }\end{array}$ & INCREMENTAL & $\begin{array}{c}\text { INCREMENTAL } \\
\text { COST }\end{array}$ & $\begin{array}{l}\text { INCKEMENTAL } \\
\text { ANNUAL HTG }\end{array}$ & $\begin{array}{l}\text { INCREMENTAL } \\
\text { ANNUAL CLG }\end{array}$ \\
\hline $\begin{array}{l}\text { E } 3 \\
C 3 \\
\text { EXTRA }\end{array}$ & $\begin{array}{l}.096 \\
.043 \\
.015\end{array}$ & $\begin{array}{r}101 \\
\$ 470 \\
\$ \quad 26\end{array}$ & $\begin{array}{l}.345 \\
.122 \\
.040\end{array}$ & $\begin{array}{l}=762 \\
\therefore 463 \\
\because 172\end{array}$ \\
\hline
\end{tabular}

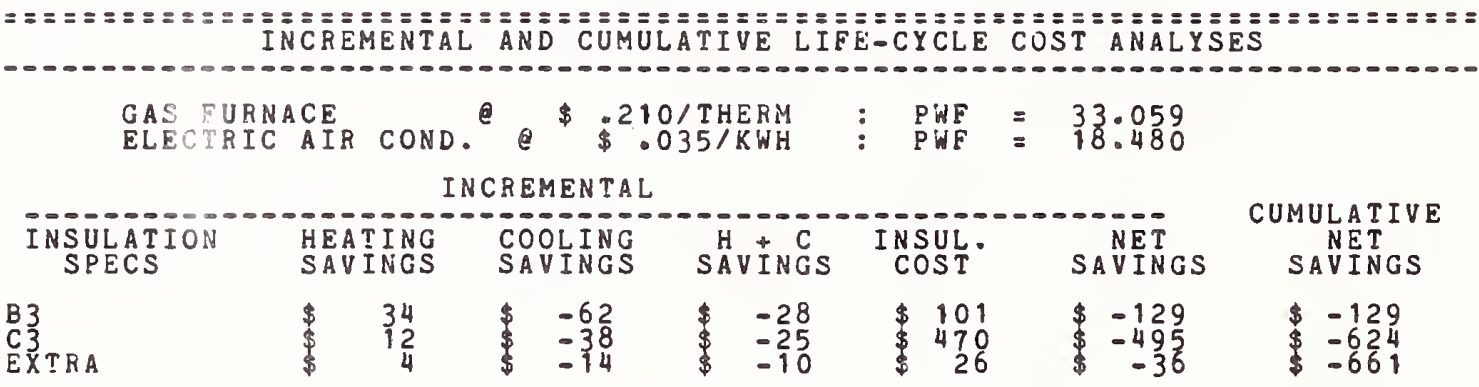

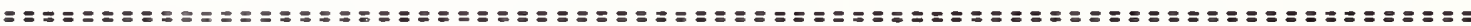

OIL EURNACE

INCREMENTAL

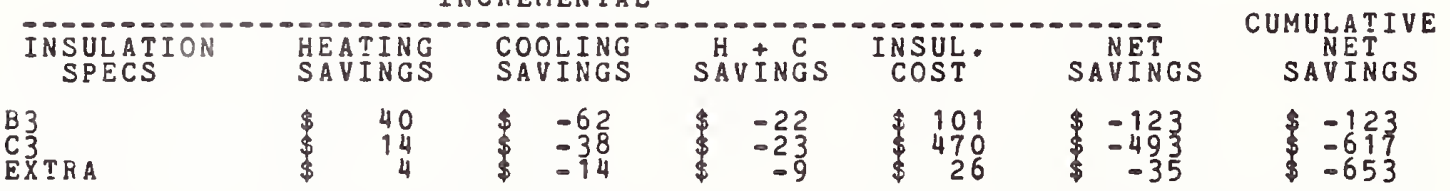

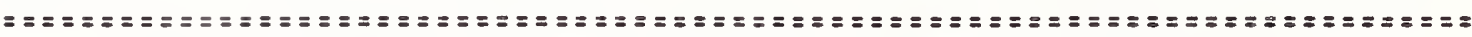

ELECTRIC FURNACE 巳 \$.035/KWH $\$$ PWF $=18.480$

INCREMENTAL

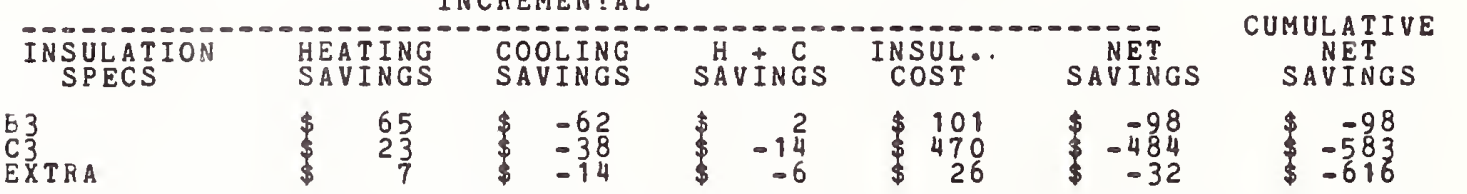

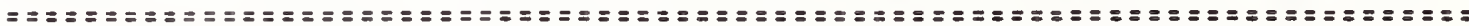

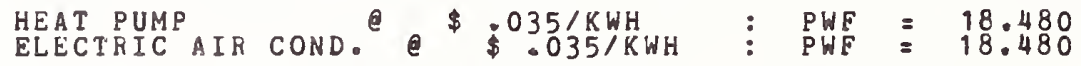

INCREMENTAL

\begin{tabular}{|c|c|c|c|c|c|c|}
\hline $\begin{array}{l}\text { INSULAT ION } \\
\text { SPECS }\end{array}$ & $\begin{array}{l}\text { HEATING } \\
\text { SAVINGS }\end{array}$ & $\begin{array}{l}\text { COOLING } \\
\text { SAVINGS }\end{array}$ & $\stackrel{H}{\text { SAVIINGS }}$ & $\begin{array}{l}\text { INSUL. } \\
\text { COST }\end{array}$ & $\begin{array}{l}\text { NET } \\
\text { SAVINGS }\end{array}$ & $\begin{array}{c}\text { CUMULATIVE } \\
\text { NET } \\
\text { SAVINGS }\end{array}$ \\
\hline XTA & $\begin{array}{r}28 \\
10 \\
3\end{array}$ & $\begin{array}{l}-65 \\
-39 \\
-74\end{array}$ & $\begin{array}{l}-37 \\
-29 \\
-11\end{array}$ & $\begin{array}{r}101 \\
\$ 470 \\
\$ 26\end{array}$ & $\begin{array}{r}-138 \\
-499 \\
-37\end{array}$ & $\begin{array}{l}-138 \\
-637 \\
-675\end{array}$ \\
\hline
\end{tabular}

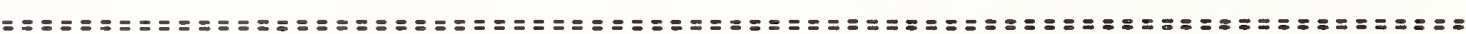


HUD 2 JACKSONVILLE ELORIDA IN BLOCK (OPEN CORE)
COOLING LOADS BASED ON WINDOWS CLOSED

\begin{tabular}{|c|c|c|c|c|}
\hline $\begin{array}{l}\text { INSULATION } \\
\text { SPECS }\end{array}$ & $\begin{array}{l}\text { U } \\
\text { VALUE }\end{array}$ & INSUL & $\begin{array}{l}\text { ANNUAL }{ }^{\text {HTG }} \\
\text { (MILLION BTU) }\end{array}$ & $\begin{array}{l}\text { ANNUAL CLG } \\
\text { (MILLION BTU) }\end{array}$ \\
\hline $\begin{array}{l}\text { BASE CASE } \\
\text { B3 } \\
\text { D } 3 \\
\text { F2 }\end{array}$ & $\begin{array}{r}.249 \\
: 153 \\
.143 \\
.091\end{array}$ & $\begin{array}{r}\$ 0 \\
101 \\
\$ 370 \\
538\end{array}$ & $\begin{array}{l}3.593 \\
2: 380 \\
2.256 \\
1.703\end{array}$ & $\begin{array}{l}32.665 \\
33: 177 \\
33: 236 \\
33.755\end{array}$ \\
\hline $\begin{array}{l}\text { INSULATION } \\
\text { SPECS }\end{array}$ & INCREMEENTAL & $\begin{array}{c}\text { INCREMENTAL } \\
\text { COST }\end{array}$ & $\begin{array}{l}\text { INCREMENTAL } \\
\text { ANNUAL HTG }\end{array}$ & $\begin{array}{l}\text { INCREMENTAL } \\
\text { ANNUAL CLG }\end{array}$ \\
\hline $\begin{array}{l}\text { B } 3 \\
\text { D } \\
\text { F } 3\end{array}$ & $\begin{array}{l}.096 \\
.090 \\
.052\end{array}$ & $\begin{array}{l}101 \\
\$ 269 \\
\$ 168\end{array}$ & $\begin{array}{r}1.213 \\
.124 \\
.553\end{array}$ & $\begin{array}{l}=.512 \\
=.059 \\
=.519\end{array}$ \\
\hline
\end{tabular}

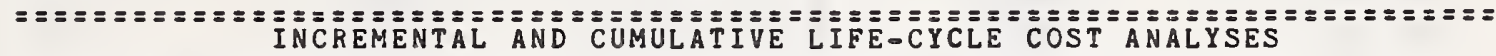

GAS FURNACE
ELECTRICAIR COND. e $e^{\$ 210 / T H E R M}: \$ P W F=33.059$

INCREMENTAL

\begin{tabular}{|c|c|c|c|c|c|c|c|c|}
\hline $\begin{array}{l}\text { INSULATION } \\
\text { SPECS }\end{array}$ & & $\begin{array}{l}\text { TING } \\
V I N G S\end{array}$ & & $\begin{array}{l}\text { LING } \\
\text { INGS }\end{array}$ & SÄVIN IC & INSUL. & SAVINGS & $\begin{array}{r}\text { CUMULATIVE } \\
\text { NVTT } \\
\text { SAVINGS }\end{array}$ \\
\hline & $\$$ & $\begin{array}{r}122 \\
12 \\
55\end{array}$ & $\$$ & $\begin{array}{l}-42 \\
-42 \\
-42\end{array}$ & $\begin{array}{l}79 \\
7 \\
12\end{array}$ & $\begin{array}{l}101 \\
\$ 269 \\
168\end{array}$ & $\begin{array}{l}\$-211 \\
\$-155 \\
\$-151\end{array}$ & $\begin{array}{l}\$-21 \\
-282 \\
-437\end{array}$ \\
\hline
\end{tabular}

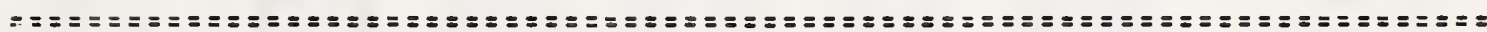

OIL FURNACE
ELECTRICAIR COND. $\$ \$ 490 / G A L L O N$

INCREMENTAL

\begin{tabular}{|c|c|c|c|c|c|c|}
\hline $\begin{array}{l}\text { INSULATION } \\
\text { SPECS }\end{array}$ & $\begin{array}{l}\text { HEATING } \\
\text { SAVINGS }\end{array}$ & $\begin{array}{l}\text { COOLING } \\
\text { SAVINGS }\end{array}$ & $\stackrel{H}{\text { SAVINGS }}$ & $\begin{array}{l}\text { INSUL. } \\
\text { COST }\end{array}$ & $\begin{array}{l}\text { NET } \\
\text { SAVINGS }\end{array}$ & SAVINGS \\
\hline & $\begin{array}{r}\quad 149 \\
\$ \quad 14 \\
\$ \quad 64\end{array}$ & $\begin{array}{rr}\$ & -42 \\
\$ & -42\end{array}$ & $\begin{array}{r}98 \\
29\end{array}$ & $\begin{array}{l}\$ 101 \\
\$ 269 \\
\$ 168\end{array}$ & $\begin{array}{l}\$-2 \\
\$=146\end{array}$ & $\begin{array}{r}-26 \\
-261 \\
-407\end{array}$ \\
\hline
\end{tabular}

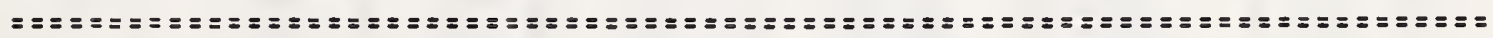
ELECTRIC FURNACE
ELECTRIC AIR COND. $\$ \$ P$ PWF INCREMENTAL

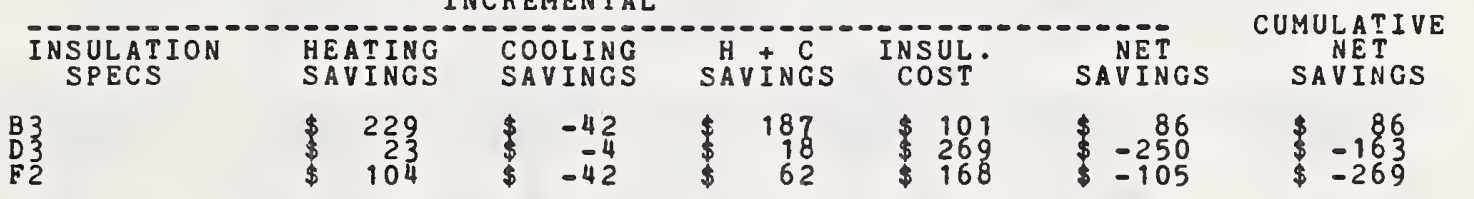

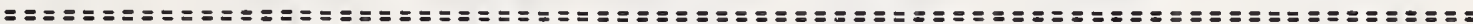
HEAT PUMP
ELECTRIC AIR COND. $e^{\$ 035 / K W H}$ INCREMENTAL

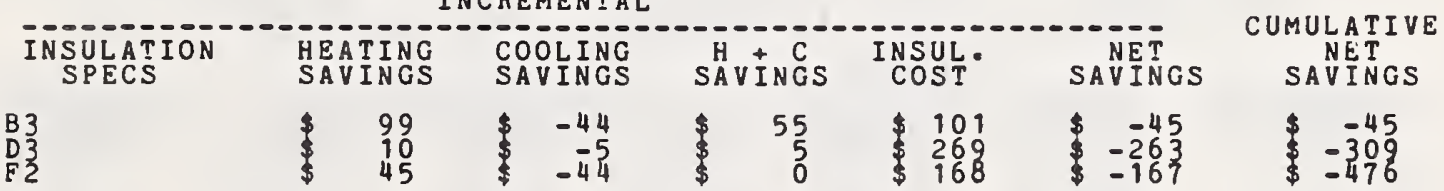

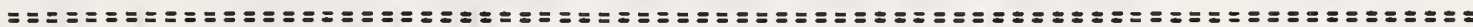


HUD 2

PHOENIX ARIZONA COOLING LOADS BASED ON WINDOWS CLOSED

8 IN BLOCK (OPEN CORE)

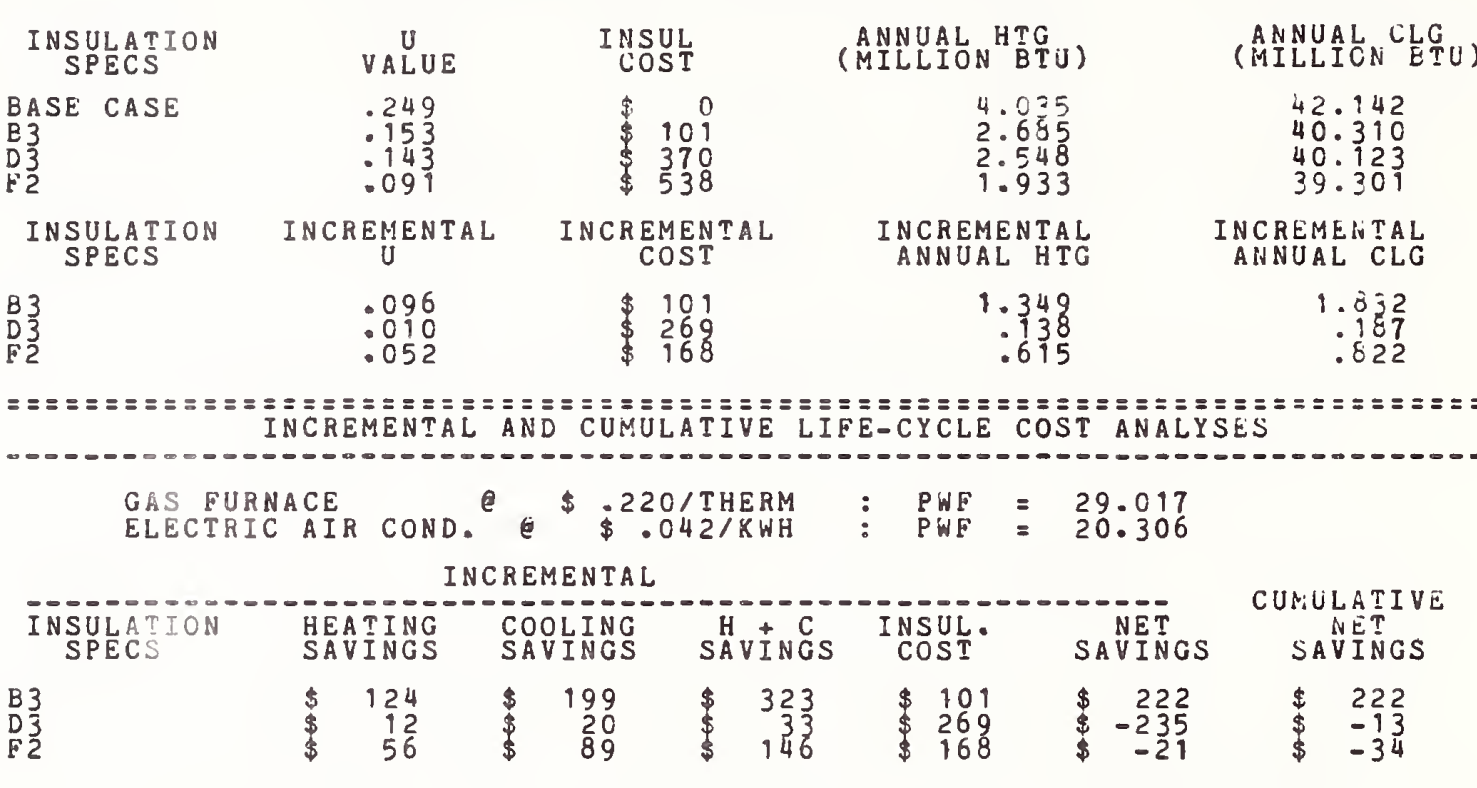

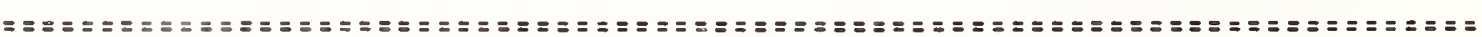

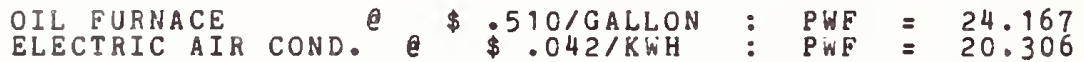

I R CREMENTAL

\begin{tabular}{|c|c|c|c|c|c|c|c|}
\hline $\begin{array}{l}\text { I HSULATION } \\
\text { SPECS }\end{array}$ & $\begin{array}{l}\text { HEATING } \\
\text { SAVINGS }\end{array}$ & $\begin{array}{l}\text { COOLING } \\
\text { SAVINGS }\end{array}$ & SÄVİ C & $\begin{array}{l}\text { INSUL. } \\
\text { COST }\end{array}$ & SAVINGS & $S A$ & $\begin{array}{l}\text { ATIVE } \\
\text { ET } \\
\text { INGS }\end{array}$ \\
\hline & $\begin{array}{r}152 \\
15 \\
69\end{array}$ & $\begin{array}{r}199 \\
20 \\
89\end{array}$ & $\begin{array}{r}351 \\
35 \\
158\end{array}$ & $\begin{array}{l}101 \\
\$ 269 \\
\$ 168\end{array}$ & $\begin{array}{r}250 \\
-233 \\
\$ \quad-9\end{array}$ & $\$$ & $\begin{array}{r}250 \\
17 \\
7\end{array}$ \\
\hline
\end{tabular}

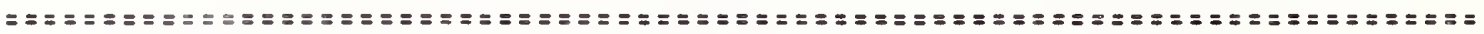

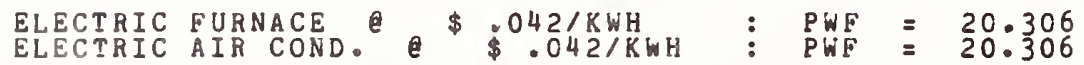

INCREMENTAL

\begin{tabular}{|c|c|c|c|c|c|c|c|}
\hline $\begin{array}{l}\text { INSULATION } \\
\text { SPECS }\end{array}$ & $\begin{array}{l}\text { HEATING } \\
\text { SAVINGS }\end{array}$ & $\begin{array}{l}\text { COOLING } \\
\text { SAVINGS }\end{array}$ & $\stackrel{H}{\text { SAVINGS }} \underset{\text { C }}{C}$ & $\begin{array}{l}\text { INSUL. } \\
\text { COST }\end{array}$ & SAVINGS & $\begin{array}{r}\text { U.HU } \\
\text { SA }\end{array}$ & $\begin{array}{l}\text { ATT IVE } \\
\text { INGS }\end{array}$ \\
\hline & $\begin{array}{r}337 \\
34 \\
153\end{array}$ & $\begin{array}{r}199 \\
20 \\
89\end{array}$ & $\begin{array}{r}536 \\
54 \\
243\end{array}$ & $\begin{array}{l}101 \\
\$ 269 \\
\$ 168\end{array}$ & $\begin{array}{r}435 \\
-214 \\
\$ \quad 75\end{array}$ & $\$$ & $\begin{array}{l}435 \\
220 \\
295\end{array}$ \\
\hline
\end{tabular}

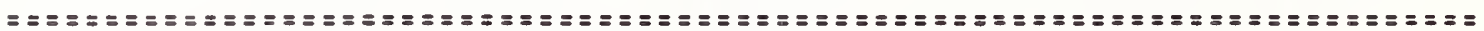

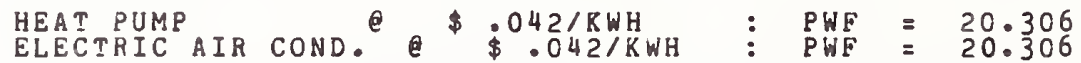

I NCREMENTAL

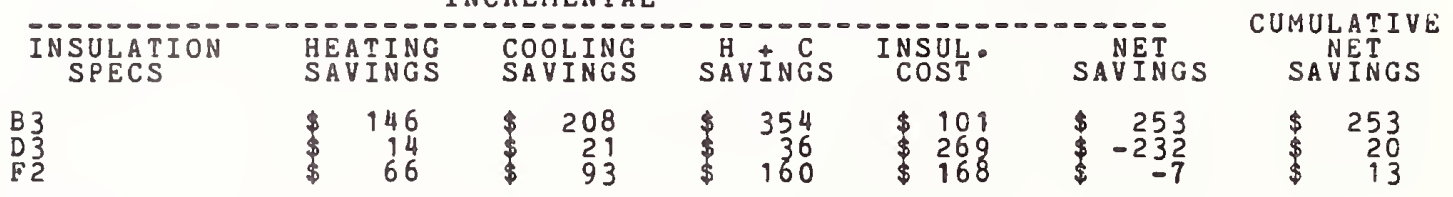

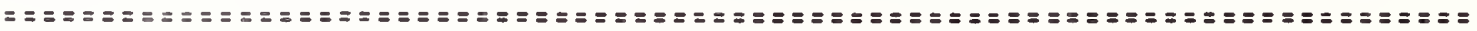


HUD2

TAMPA FLORIDA

1200 SQ FT HOUSE

8 IN BLOCK (OPEN CORE) COOLING LOADS BASED ON WINDOWS CLOSED

\begin{tabular}{|c|c|c|c|c|}
\hline $\begin{array}{l}\text { INSULATION } \\
\text { SPECS }\end{array}$ & VALUE & $\begin{array}{l}\text { INSUL } \\
\text { COST }\end{array}$ & $\begin{array}{l}\text { ANNUAL }{ }^{\text {HTG }} \\
\text { MILLION }\end{array}$ & $\begin{array}{l}\text { ANNUAL CLG } \\
\text { (MILLION BTU) }\end{array}$ \\
\hline $\begin{array}{l}\text { BASE CASE } \\
\text { B3 } \\
\text { D3 } \\
\text { F2 }\end{array}$ & $\begin{array}{r}.249 \\
.153 \\
.143 \\
.091\end{array}$ & $\begin{array}{rr}\$ & 0 \\
101 \\
370 \\
538\end{array}$ & $\begin{array}{r}.850 \\
.505 \\
.471 \\
.333\end{array}$ & $\begin{array}{l}38.104 \\
38.866 \\
38.950 \\
39.547\end{array}$ \\
\hline $\begin{array}{l}\text { INSULATION } \\
\text { SPECS }\end{array}$ & IN CREM & $\begin{array}{c}\text { INCREMENTAL } \\
\text { COST }\end{array}$ & $\begin{array}{l}\text { INCREMENTAL } \\
\text { ANNUAL HTG }\end{array}$ & $\begin{array}{l}\text { INCREMENTAL } \\
\text { ANNUAL CLG }\end{array}$ \\
\hline $\begin{array}{l}\text { B } 3 \\
\text { D } 3 \\
\text { F2 }\end{array}$ & $\begin{array}{l}.096 \\
.010 \\
.052\end{array}$ & $\begin{array}{l}101 \\
\$ 269 \\
\$ 168\end{array}$ & $\begin{array}{r}.345 \\
.035 \\
.138\end{array}$ & $\begin{array}{l}-.762 \\
\therefore .084 \\
=.597\end{array}$ \\
\hline
\end{tabular}

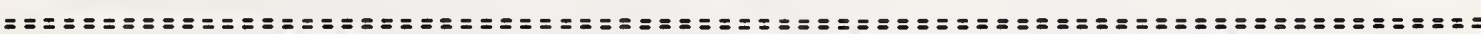
INCREMENTAL AND CUMULATIVE LIFE-CYCLE CUST ANALYSES

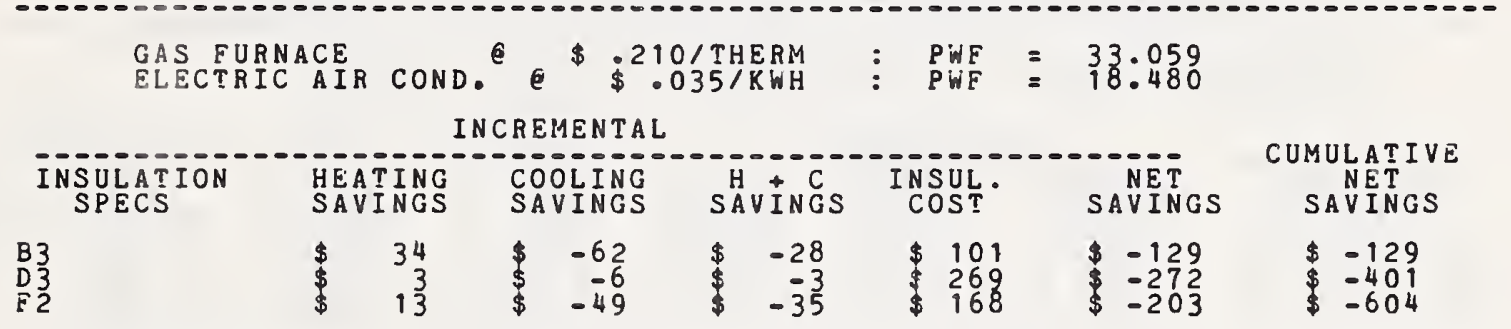

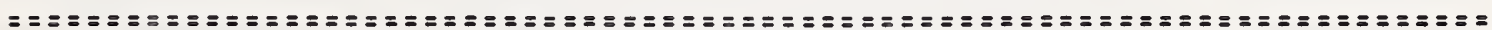

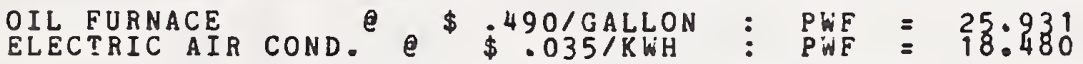

INCREMENTAL

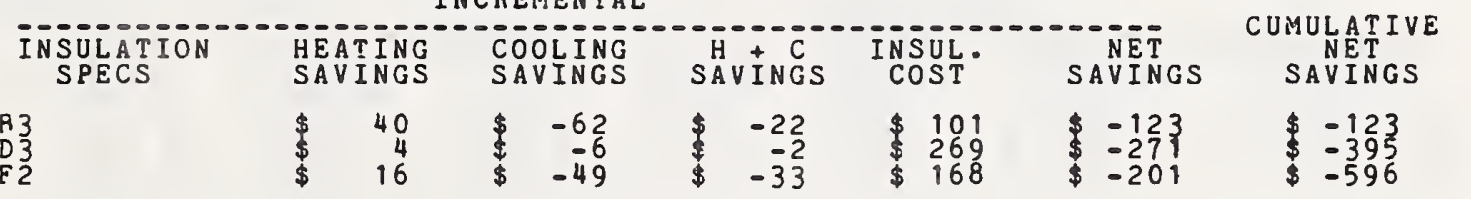

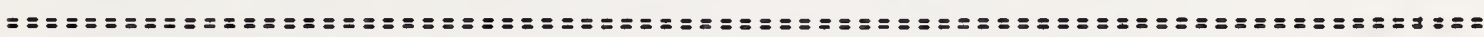

ELECTRIC FURNACE
ELECTRIC AIR COND. $e^{\$ 035 / K W H}$

INCREMENTAL

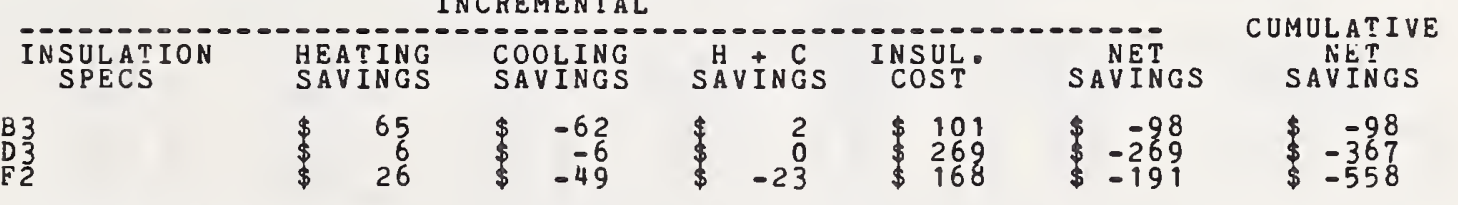

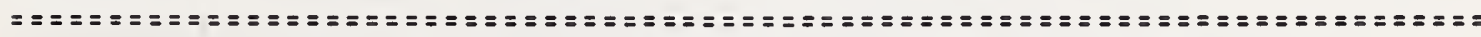
HEAT PUMP
ELECTRIC AIR COND.

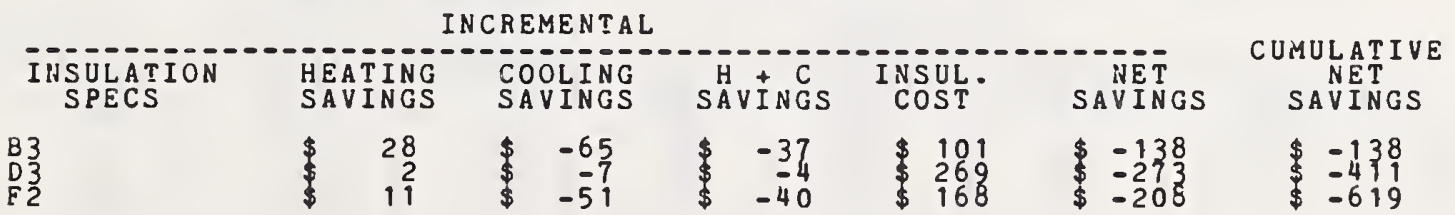

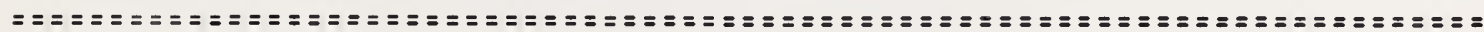


HUD 3

JACKSONVILLE FLORIDA 1200 SQ FT HOUSE IN BLOCK (PERLITE FILLED) COOLING LOADS BASED ON WINDOWS CLOSED

\begin{tabular}{|c|c|c|c|c|}
\hline $\begin{array}{l}\text { INSULATION } \\
\text { SPECS SON }\end{array}$ & VALUE & $\begin{array}{l}\text { INSUL } \\
\text { COST }\end{array}$ & $\begin{array}{l}\text { ANNUAL HTG } \\
\text { (MILLION BTU) }\end{array}$ & $\begin{array}{l}\text { ANNUAL CLG } \\
\text { (MILLION BTU) }\end{array}$ \\
\hline $\begin{array}{l}\text { BASE CASE } \\
\text { CORES EILLED } \\
\text { B } 4 \\
\text { C4 }\end{array}$ & $\begin{array}{l}.249 \\
: 144 \\
1004 \\
.082\end{array}$ & $\begin{array}{r}530 \\
\$ 238 \\
6109 \\
\$ 1109\end{array}$ & $\begin{array}{l}3.608 \\
2.026 \\
1.538 \\
1.368\end{array}$ & $\begin{array}{l}32.697 \\
32: 798 \\
32: 971 \\
33.392\end{array}$ \\
\hline $\begin{array}{l}\text { INSULATION } \\
\text { SPECS }\end{array}$ & INCREMENTAL & $\begin{array}{c}\text { INCREMENTAL } \\
\operatorname{COST}\end{array}$ & $\begin{array}{l}\text { INCREMENTAL } \\
\text { ANNUAL HTG }\end{array}$ & $\begin{array}{l}\text { INCREMENTAL } \\
\text { ANNUAL CLG }\end{array}$ \\
\hline $\begin{array}{l}\text { C.ORES FILLED } \\
\text { B } 4 \\
\text { C4 }\end{array}$ & $\begin{array}{l}.108 \\
.037 \\
.022\end{array}$ & $\begin{array}{l}538 \\
\$ 101 \\
\$ \$ \$ \$ \\
\$ 470\end{array}$ & $\begin{array}{r}1.581 \\
: 489 \\
.169\end{array}$ & $\begin{array}{l}\because 101 \\
\because 173 \\
\because 420\end{array}$ \\
\hline
\end{tabular}

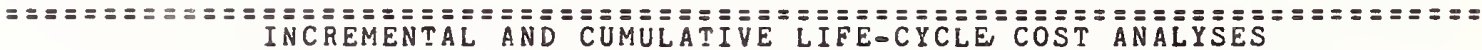

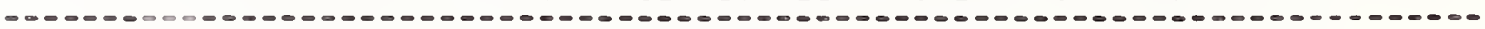

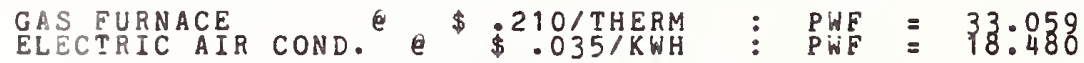

INCREMENTAL

\begin{tabular}{|c|c|c|c|c|c|c|}
\hline $\begin{array}{l}\text { IHSULATION } \\
\text { SPECS }\end{array}$ & $\begin{array}{l}\text { HEAT ING } \\
\text { SAVINGS }\end{array}$ & $\begin{array}{l}\text { COOLING } \\
\text { SAVINGS }\end{array}$ & SAVINGS & $\begin{array}{l}\text { INSUL: } \\
\text { COST }\end{array}$ & SAVINGS & $\begin{array}{c}\text { CUMULATIVE } \\
\text { NET } \\
\text { SAVINGS }\end{array}$ \\
\hline ORES FILLED & $\begin{array}{r}159 \\
49 \\
17\end{array}$ & $\begin{array}{l}\$-8 \\
\$-14 \\
-34\end{array}$ & $\begin{array}{r}150 \\
34 \\
-17\end{array}$ & $\begin{array}{l}538 \\
\$ 101 \\
\$ 470\end{array}$ & $\begin{array}{r}-387 \\
-66 \\
-487\end{array}$ & $\begin{array}{l}-387 \\
=453 \\
-940\end{array}$ \\
\hline
\end{tabular}

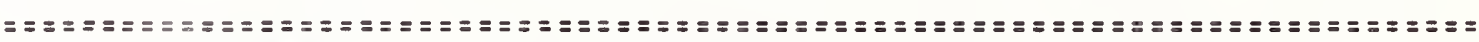

OIL FURNACE
ELECTRICAIR COND. e \$ $\$$ \$ $\$$.035/KWH

INCREMENTAL

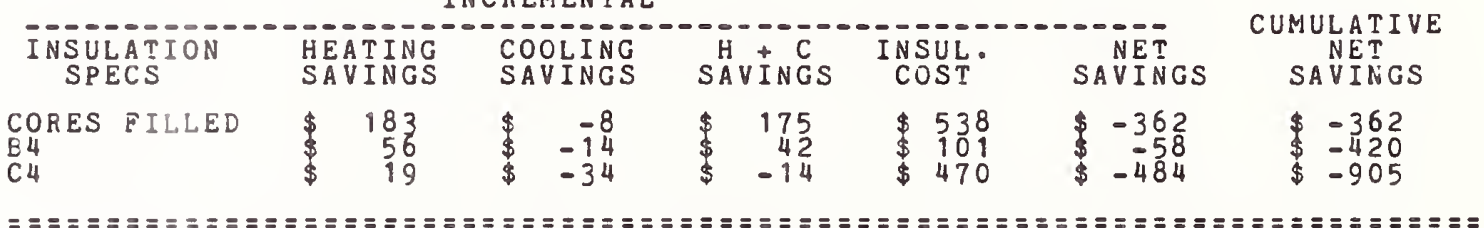

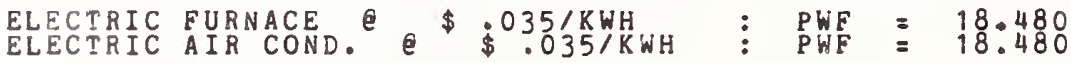

INCREMENTAL

\begin{tabular}{|c|c|c|c|c|c|c|}
\hline $\begin{array}{l}\text { INSULATION } \\
\text { SPECS }\end{array}$ & $\begin{array}{l}\text { HEATING } \\
\text { SAVINGS }\end{array}$ & $\begin{array}{l}\text { COOLING } \\
\text { SAVINGS }\end{array}$ & $\begin{array}{l}\text { HU } \\
\text { SAVINGS }\end{array}$ & $\begin{array}{l}\text { INSUL. } \\
\text { COST }\end{array}$ & $\begin{array}{l}\text { NET } \\
\text { SAVINGS }\end{array}$ & $\begin{array}{l}\text { CUMULATIVE } \\
\text { NET } \\
\text { SAVINGS }\end{array}$ \\
\hline ES FILLED & $\begin{array}{r}299 \\
\$ \\
\$ \\
\$ 22\end{array}$ & $\begin{array}{l}\$-14 \\
\$-34\end{array}$ & $\begin{array}{r}291 \\
78 \\
-2\end{array}$ & $\begin{array}{l}538 \\
\$ 101 \\
\$ 470\end{array}$ & $\begin{array}{r}-246 \\
\$-22 \\
\$-472\end{array}$ & $\begin{array}{l}\$-246 \\
=269 \\
\$-741\end{array}$ \\
\hline
\end{tabular}

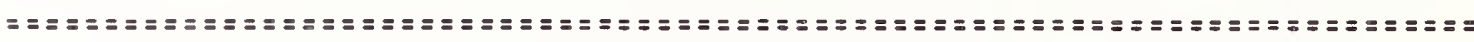

HEATPUMP PIR COND. \$ $\$$ PWF $=18.480$

INCREMENTAL

\begin{tabular}{|c|c|c|c|c|c|c|}
\hline $\begin{array}{l}\text { INSULATION } \\
\text { SPECS }\end{array}$ & $\begin{array}{l}\text { HEAT ING } \\
\text { SAVINGS }\end{array}$ & $\begin{array}{l}\text { COOLING } \\
\text { SAVINGS }\end{array}$ & SÄVINGS & INSUL. & SAVINGS & $\begin{array}{c}\text { CUMULAT IVE } \\
\text { NVET } \\
\text { SAVINGS }\end{array}$ \\
\hline $\begin{array}{l}\text { CORES FILLED } \\
\text { B } 4 \\
\text { C } 4\end{array}$ & $\begin{array}{l}\$ 130 \\
\$ \\
\$ \\
\$ 13\end{array}$ & $\begin{array}{ll}\$ & -8 \\
\$ & -14 \\
& -36\end{array}$ & $\begin{array}{r}121 \\
25 \\
-22\end{array}$ & $\begin{array}{l}\$ 538 \\
\$ 101 \\
\$ 470\end{array}$ & $\begin{array}{r}-416 \\
-75 \\
\$-492\end{array}$ & $\begin{array}{l}\$-416 \\
-492 \\
\$-984\end{array}$ \\
\hline
\end{tabular}

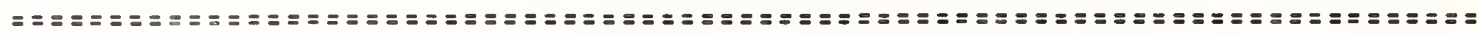


HUD 3

PHOENIX ARIZONA

1200 SO FT HOUSE

8 IN BLOCK (PERLITE FILLED) COOLING LOADS BASED ON WINDOWS CLOSED

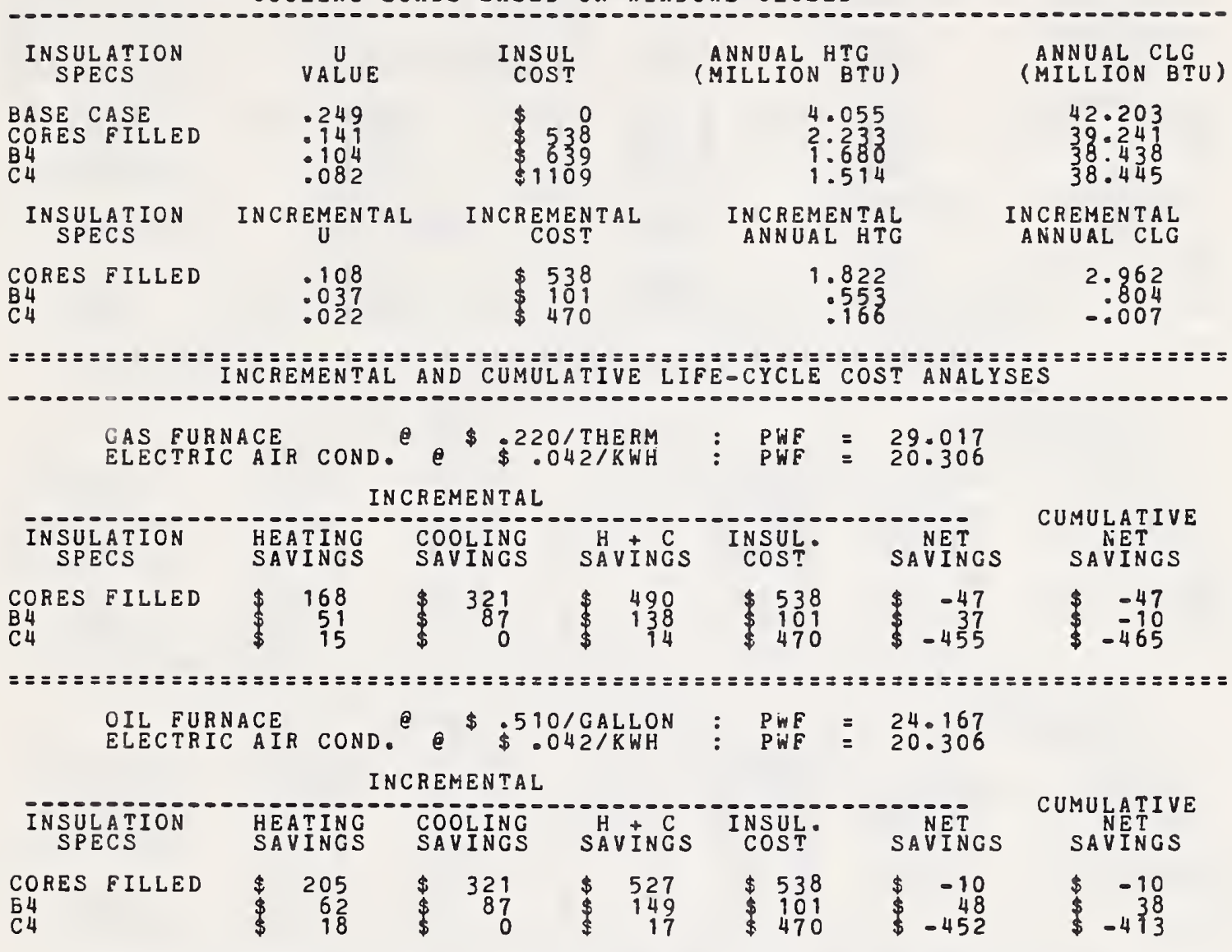

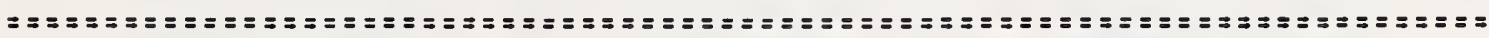

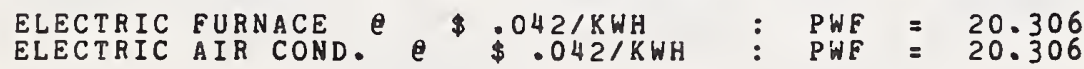

INCREMENTAL

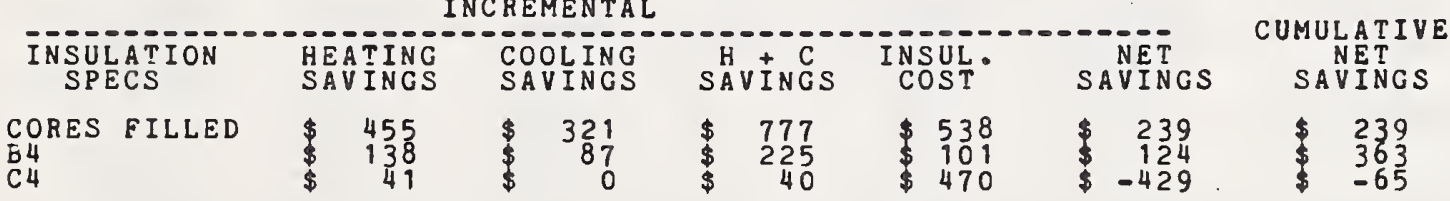

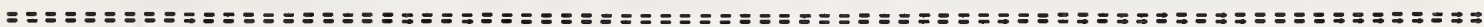
HEAT PUMP
ELECTRIC AIR COND. e $\$ \$ .042 / K W H$
$\$ P W H$

INCREMENTAL

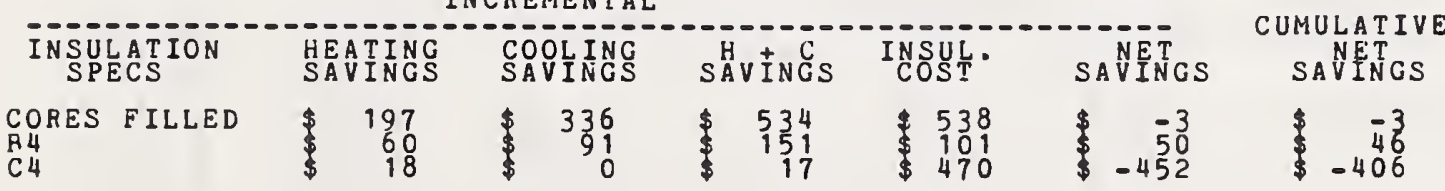




\begin{tabular}{|c|c|c|c|c|}
\hline & $\begin{aligned} \text { TAMPA } & \text { EL } \\
\text { COOLING } & \text { LO }\end{aligned}$ & $\begin{array}{l}\text { DA } \\
1200 \text { SQ FT } \\
\text { BASED ON WI }\end{array}$ & $\begin{array}{l}{ }^{8} \text { IN BLOCK } \\
\text { OUSE } \\
\text { DOWS CLOSED }\end{array}$ & (PERLITE FILLED) \\
\hline $\begin{array}{l}\text { INSUL ATION } \\
\text { SPECS }\end{array}$ & $\begin{array}{c}\text { U } \\
\text { VALUE }\end{array}$ & $\begin{array}{l}\text { INSUL } \\
\text { COST }\end{array}$ & $\begin{array}{l}\text { ANNUAL HTG } \\
\text { (MILLION BTU) }\end{array}$ & $\begin{array}{l}\text { ANNUAL CLG } \\
\text { (MILLION BTU) }\end{array}$ \\
\hline $\begin{array}{l}\text { BASE CASE } \\
\text { CORES FILLED } \\
\text { B } 4 \\
\text { C } 4\end{array}$ & $\begin{array}{r}.249 \\
.149 \\
.104 \\
.082\end{array}$ & $\begin{array}{r}\$ \\
\$ 338 \\
\$ 639 \\
\$ 1109\end{array}$ & $\begin{array}{r}.854 \\
.415 \\
.281 \\
.239\end{array}$ & $\begin{array}{l}38.133 \\
38.568 \\
38.841 \\
39.289\end{array}$ \\
\hline $\begin{array}{l}\text { INSULATION } \\
\text { SPECS }\end{array}$ & IN CRE $\underset{U}{M E N T A L}$ & $\begin{array}{c}\text { INCREMENTAL } \\
\text { COST }\end{array}$ & $\begin{array}{l}\text { INCREMENTAL } \\
\text { ANNUAL HTG }\end{array}$ & $\begin{array}{l}\text { INCREMENTAL } \\
\text { ANNUAL CLG }\end{array}$ \\
\hline $\begin{array}{l}\text { COKES FILLED } \\
\text { B } 4 \\
\text { C } 4\end{array}$ & $\begin{array}{l}.108 \\
.037 \\
.022\end{array}$ & $\begin{array}{l}\$ 538 \\
\$ 101 \\
\$ 470\end{array}$ & $\begin{array}{l}.439 \\
.134 \\
.042\end{array}$ & $\begin{array}{l}-.435 \\
\therefore .273 \\
-.448\end{array}$ \\
\hline
\end{tabular}

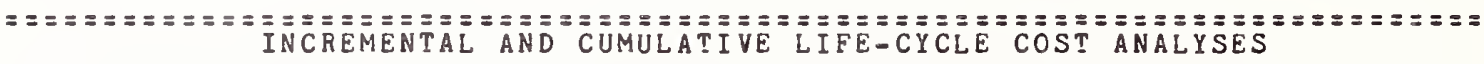

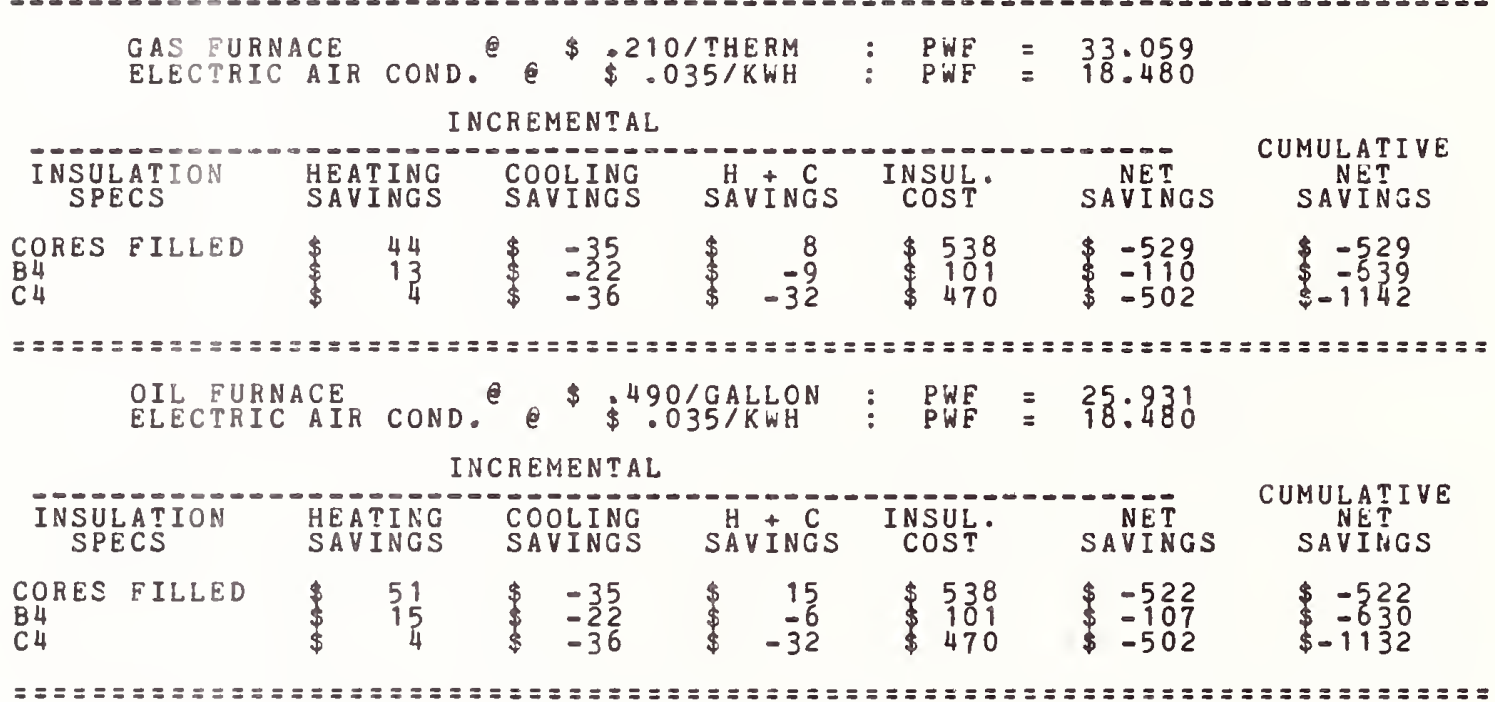

ELECTRIC FURNACE
ELECTRIC AIR COND. $\$ \$ 035 / K W H$
$\$ P W H F$

INCREMENTAL

\begin{tabular}{|c|c|c|c|c|c|c|}
\hline $\begin{array}{l}\text { NSULATION } \\
\text { SPECS }\end{array}$ & $\begin{array}{l}\text { HEAT ING } \\
\text { SAVINGS }\end{array}$ & $\begin{array}{l}\text { COOL I NG } \\
\text { SAVINGS }\end{array}$ & $\begin{array}{l}\text { H } \\
\text { SAVINGS }\end{array}$ & $\begin{array}{l}\text { INSUL. } \\
\text { COST }\end{array}$ & $\begin{array}{l}\text { NET } \\
\text { SAVINGS }\end{array}$ & $\begin{array}{l}\text { CUMULATIVE } \\
\text { NET } \\
\text { SAVINGS }\end{array}$ \\
\hline S FILLED & $\begin{array}{l}83 \\
25 \\
7\end{array}$ & $\begin{array}{l}\$-35 \\
\$-22 \\
\$-36\end{array}$ & $\begin{array}{r}47 \\
\$ \quad-28\end{array}$ & $\begin{array}{l}538 \\
\$ 101 \\
\$ 470\end{array}$ & $\begin{array}{r}-490 \\
-98 \\
-498\end{array}$ & $\begin{array}{r}-490 \\
-588 \\
-1087\end{array}$ \\
\hline
\end{tabular}

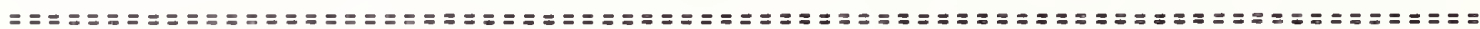

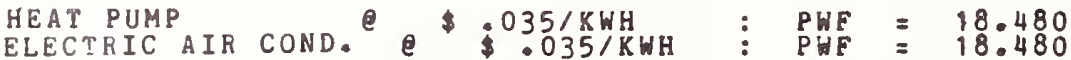
INCREMENTAL

\begin{tabular}{|c|c|c|c|c|c|c|}
\hline $\begin{array}{l}\text { INSULATION } \\
\text { SPECS }\end{array}$ & $\begin{array}{l}\text { HEATING } \\
\text { SAVINGS }\end{array}$ & $\begin{array}{l}\text { COOLING } \\
\text { SAVINGS }\end{array}$ & SAVINGS & $\begin{array}{l}\text { INSUL: } \\
\text { COST }\end{array}$ & $\begin{array}{l}\text { NET } \\
\text { SAVINGS }\end{array}$ & $\begin{array}{c}\text { CUMULATIVE } \\
\text { NET } \\
\text { SAVINGS }\end{array}$ \\
\hline $\int_{4} R E S$ & $\begin{array}{l}36 \\
31 \\
3\end{array}$ & $\begin{array}{l}\$=37 \\
\$-33 \\
\$-38\end{array}$ & $\begin{array}{r}-12 \\
-12 \\
-35\end{array}$ & $\begin{array}{l}538 \\
\$ 181 \\
\$ 470\end{array}$ & $\begin{array}{l}-539 \\
=173 \\
=505\end{array}$ & $\begin{array}{r}-539 \\
=-652 \\
\$-1157\end{array}$ \\
\hline
\end{tabular}

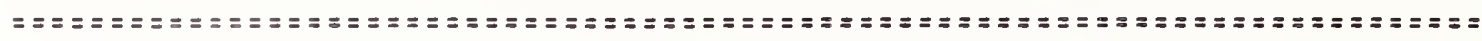


HUD 4

JACKSONVILLE FLORIDA 200 SO FT HOUSE 8 IN BLOCK (PERLITE FILLED) COOLING LOADS BASED ON WINDOWS CLOSED

\begin{tabular}{|c|c|c|c|c|}
\hline $\begin{array}{l}\text { INSULATION } \\
\text { SPECS }\end{array}$ & $\stackrel{U}{\text { VALUE }}$ & $\begin{array}{l}\text { INSUL } \\
\text { COST }\end{array}$ & $\begin{array}{l}\text { ANNUAL HTG } \\
\text { (MILLION }\end{array}$ & $\begin{array}{l}\text { ANNUAL CLG } \\
\text { (MILLION BTU) }\end{array}$ \\
\hline $\begin{array}{l}\text { BASE CASE } \\
\text { CORES FILLED } \\
\text { B4 } \\
\text { D } 4 \\
\text { F5 }\end{array}$ & $\begin{array}{l}.249 \\
.141 \\
.104 \\
.099 \\
.071\end{array}$ & $\begin{array}{r}\$ 0 \\
\$ 538 \\
\$ 639 \\
\$ 908 \\
\$ 1076\end{array}$ & $\begin{array}{l}3.608 \\
2.026 \\
1.538 \\
1.499 \\
1.296\end{array}$ & $\begin{array}{l}32.697 \\
32: 798 \\
32.971 \\
33.067 \\
33.608\end{array}$ \\
\hline $\begin{array}{l}\text { INSULATION } \\
\text { SPECS }\end{array}$ & INCREMENTAL & $\begin{array}{c}\text { INCREMENTAL } \\
\text { COST }\end{array}$ & $\begin{array}{l}\text { INCREMENTTEL } \\
\text { ANNUAL HTG }\end{array}$ & $\begin{array}{l}\text { INCKEUENTAL } \\
\text { ANNUAL CLG }\end{array}$ \\
\hline $\begin{array}{l}\text { CORES FILLED } \\
\text { B4 } \\
\text { D4 } \\
\text { F5 }\end{array}$ & $\begin{array}{r}.108 \\
.037 \\
.005 \\
.028\end{array}$ & $\begin{array}{l}538 \\
\$ 101 \\
\$ 269 \\
\$ 168\end{array}$ & $\begin{array}{r}1.581 \\
.489 \\
.038 \\
.203\end{array}$ & $\begin{array}{l}-.109 \\
: .173 \\
\therefore .096 \\
-.541\end{array}$ \\
\hline \multicolumn{5}{|c|}{ 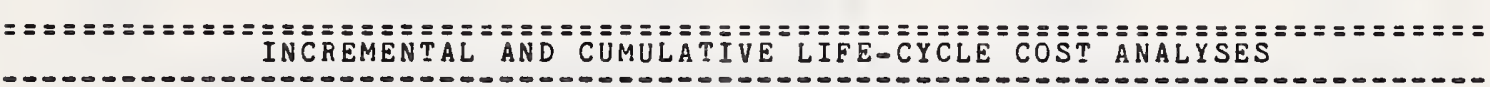 } \\
\hline
\end{tabular}

INCREMENTAL

\begin{tabular}{|c|c|c|c|c|c|c|}
\hline $\begin{array}{l}\text { INSULATION } \\
\text { SPECS }\end{array}$ & $\begin{array}{l}\text { HEATING } \\
\text { SAVINGS }\end{array}$ & $\begin{array}{l}\text { COOLING } \\
\text { SAVINGS }\end{array}$ & SAVINGS & $\begin{array}{l}\text { INSUL. } \\
\text { COST }\end{array}$ & SAVINTES & $\begin{array}{l}\text { CUMULATIVE } \\
\text { NET } \\
\text { SAVINGS }\end{array}$ \\
\hline $\begin{array}{l}\text { CORES FILLED } \\
\text { B } 4 \\
\text { D } 4 \\
55\end{array}$ & $\begin{array}{r}\$ \\
\$ \quad 49 \\
\$ \\
\$ \quad 20\end{array}$ & $\begin{array}{r}-8 \\
-14 \\
\$-44\end{array}$ & $\begin{array}{r}150 \\
34 \\
-44 \\
-24\end{array}$ & $\begin{array}{l}538 \\
\$ 101 \\
\$ 269 \\
\$ 168\end{array}$ & $\begin{array}{r}-387 \\
-66 \\
-273 \\
-192\end{array}$ & $\begin{array}{l}-387 \\
-453 \\
-726 \\
-918\end{array}$ \\
\hline
\end{tabular}

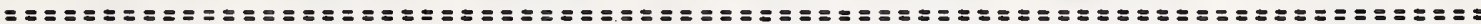
OIL FURNACE
ELECTRICAIR COND. e \$490/GALLON $\$ P W F=25.931$ INCREMENTAL

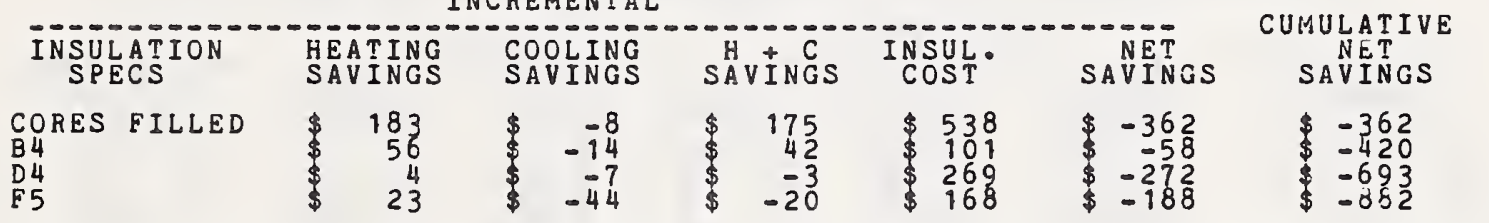

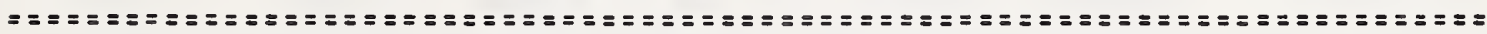
ELECTRIC FURNACE C $\$$ ELTTIC AIR COND. $\$ 35 / K W H$
ELECTH $\$ P W F=18.480$

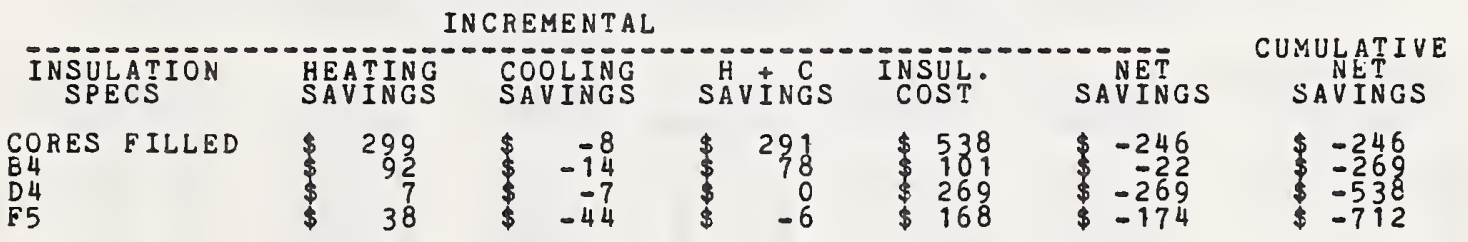

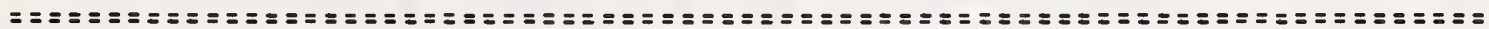
HEAT PUMP
ELECTRICAIR COND. e $\$$ \$ $\$ 35 / K W H$

INCREMENTAL

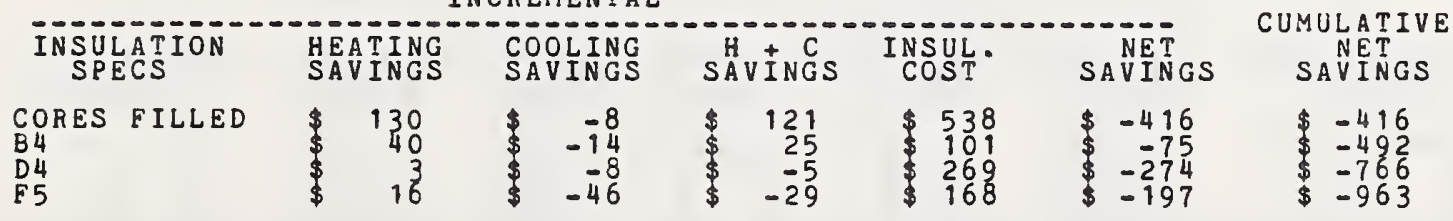


HUD 4 PHOENIX ARIZONA
COOLING LOADS BAS BLOCK (PERLITE FILLED)
BON FT HOUSE WINDOWS CLOSED

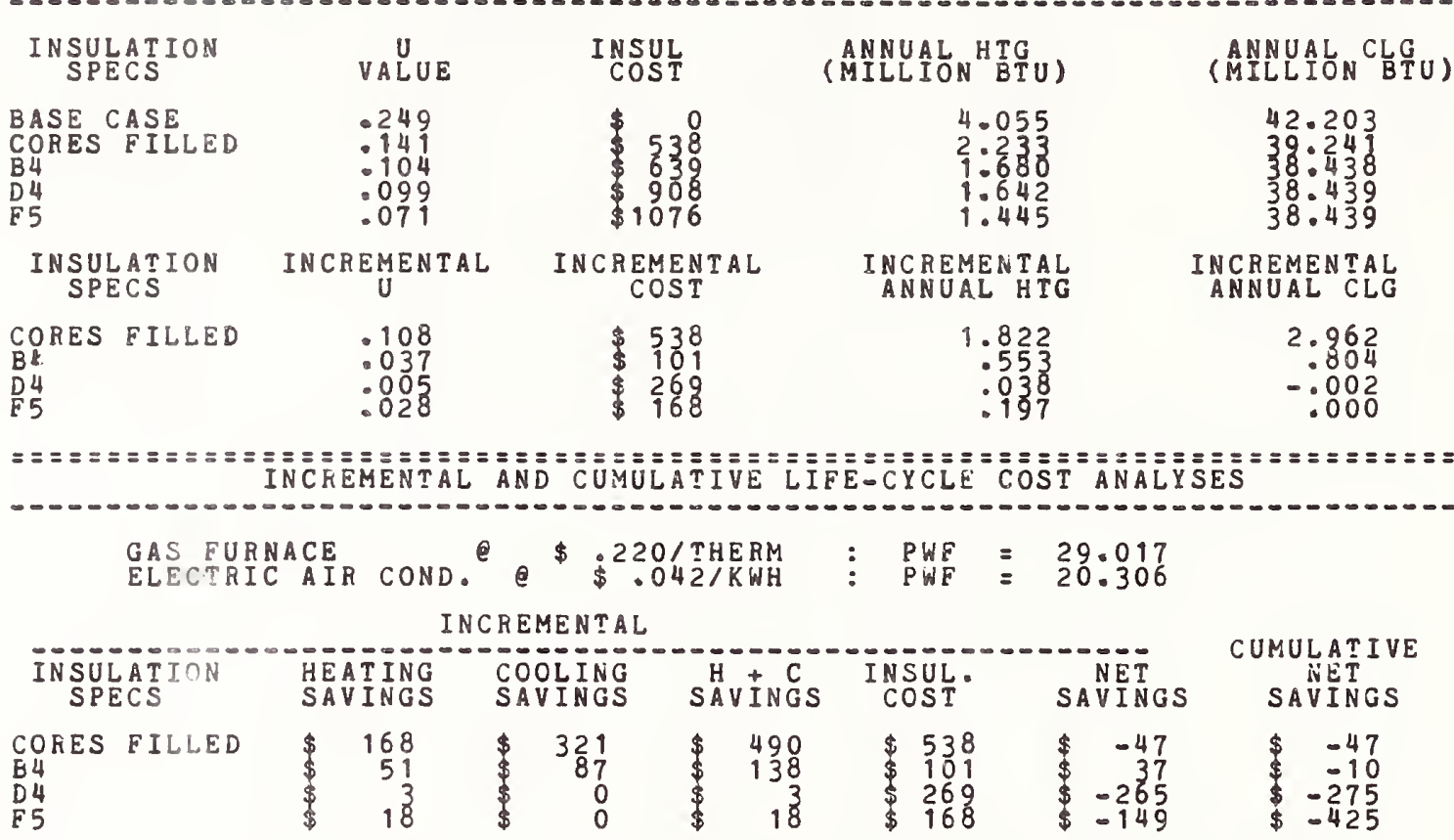

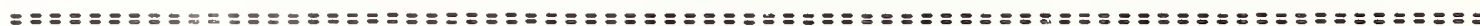

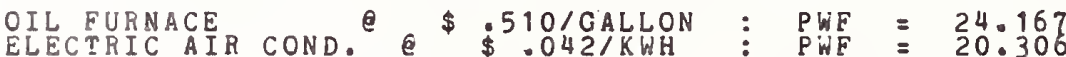

INCREMENTAL

\begin{tabular}{|c|c|c|c|c|c|c|}
\hline $\begin{array}{l}\text { INSULAT ION } \\
\text { SPECS }\end{array}$ & $\begin{array}{l}\text { HEATING } \\
\text { SAVINGS }\end{array}$ & $\begin{array}{l}\text { COOLING } \\
\text { SAVINGS }\end{array}$ & SAVINGS & $\begin{array}{l}\text { INSUL. } \\
\text { COST }\end{array}$ & SAVINGS & $\begin{array}{c}\text { CUMULATIVE } \\
\text { NETT } \\
\text { SAVINGS }\end{array}$ \\
\hline RES FILLED & $\begin{array}{r}205 \\
62 \\
4 \\
22\end{array}$ & $\begin{array}{r}\quad 321 \\
\$ \quad 87 \\
\$ \\
\$\end{array}$ & $\begin{array}{r}527 \\
\$ \quad 149 \\
\$ \quad 22\end{array}$ & $\begin{array}{l}\$ 538 \\
\$ 101 \\
\$ 269 \\
\$ 168\end{array}$ & $\begin{array}{rr}\$ & -10 \\
\$ & 48 \\
& -264 \\
\$ & -145\end{array}$ & 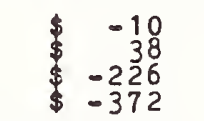 \\
\hline
\end{tabular}

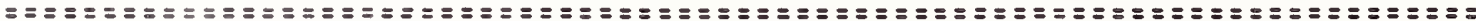

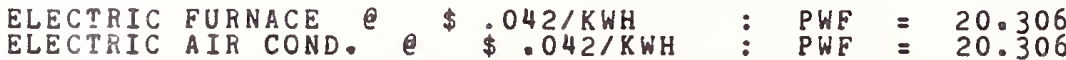

IN CREMENTAL

\begin{tabular}{|c|c|c|c|c|c|c|c|c|c|}
\hline $\begin{array}{l}\text { INSULATION } \\
\text { SPECS }\end{array}$ & $\begin{array}{l}H E A T I N G \\
\text { SAVINGS }\end{array}$ & & INGS & & INGS & $\begin{array}{l}\text { INSUL. } \\
\text { COST }\end{array}$ & $\begin{array}{l}\text { NEET } \\
\text { SAVINGS }\end{array}$ & $\begin{array}{r}\text { CUMU } \\
\text { SA }\end{array}$ & $\begin{array}{l}\text { AT IVE } \\
\text { VINGS }\end{array}$ \\
\hline $\begin{array}{l}\text { CORES FILLED } \\
\text { E4 } \\
\text { D4 } \\
\text { F5 }\end{array}$ & $\begin{array}{r}\quad 455 \\
\quad 138 \\
\$ \quad 99 \\
\$ \quad 49\end{array}$ & $\begin{array}{l}\$ \\
\$ \\
\$\end{array}$ & $\begin{array}{r}321 \\
87 \\
0 \\
0\end{array}$ & $\begin{array}{l}\$ \\
\$ \\
\$\end{array}$ & $\begin{array}{r}777 \\
225 \\
49 \\
49\end{array}$ & $\begin{array}{l}\$ 538 \\
\$ 101 \\
\$ 269 \\
\$ 168\end{array}$ & $\begin{array}{r}239 \\
124 \\
-259 \\
-118\end{array}$ & $\begin{array}{l}\$ \\
\$\end{array}$ & $\begin{array}{r}239 \\
363 \\
103 \\
-14\end{array}$ \\
\hline
\end{tabular}

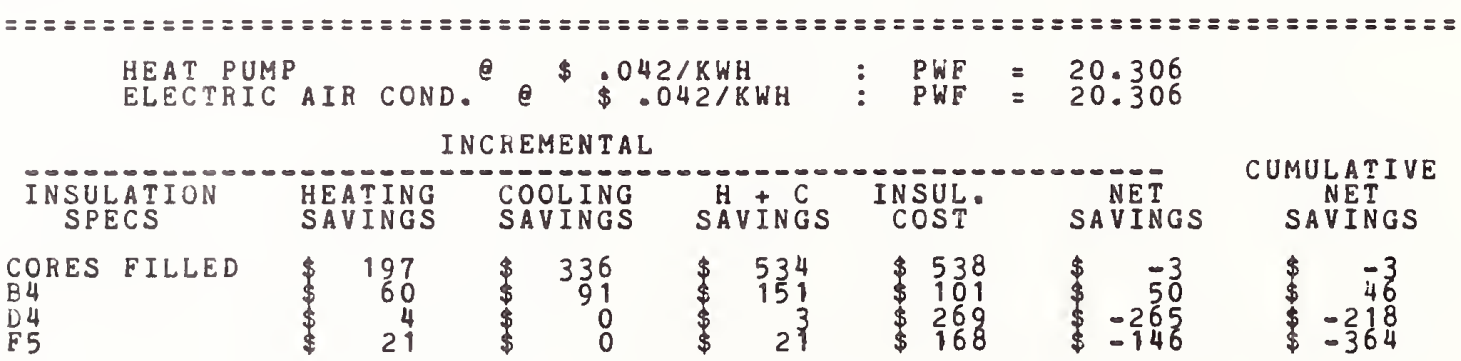

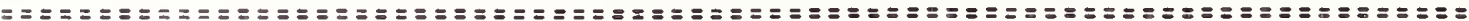


HUD 4

TAMPA FLORIDA 1200 SQ FT HOUSE 8 IN BLOCK (PERLITE FILLEC) COOLING LOADS BASED ON WINDOWS CLOSED

\begin{tabular}{|c|c|c|c|c|}
\hline $\begin{array}{l}\text { INSULATION } \\
\text { SPECS }\end{array}$ & VALUE & $\begin{array}{l}\text { INSUL } \\
\text { COST }\end{array}$ & $\begin{array}{l}\text { ANNUAL HTG } \\
\text { (MILLION BTU) }\end{array}$ & $\begin{array}{l}\text { ANNUAL CLG } \\
\text { (MILLION BTU) }\end{array}$ \\
\hline $\begin{array}{l}\text { BASE CASE } \\
\text { CORES FILLED } \\
\text { B4 } \\
\text { D } 4 \\
\text { F5 }\end{array}$ & $\begin{array}{l}.249 \\
: 149 \\
: 104 \\
.099 \\
.071\end{array}$ & 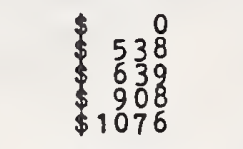 & $\begin{array}{l}.854 \\
.415 \\
.281 \\
.271 \\
.223\end{array}$ & $\begin{array}{l}38.133 \\
38: 568 \\
38: 841 \\
38: 943 \\
39.507\end{array}$ \\
\hline $\begin{array}{l}\text { INSULATION } \\
\text { SPECS }\end{array}$ & INCREM & $\begin{array}{c}\text { INCREMENTAL } \\
\text { COST }\end{array}$ & $\begin{array}{l}\text { INCREMENTAL } \\
\text { ANNUAL HTG }\end{array}$ & $\begin{array}{l}\text { INCREMENTAL } \\
\text { ANNUAL CLG }\end{array}$ \\
\hline $\begin{array}{l}\text { CORES FILLED } \\
\text { B } 4 \\
\text { D } 4 \\
\text { F5 }\end{array}$ & $\begin{array}{r}.108 \\
.037 \\
005 \\
.028\end{array}$ & $\begin{array}{l}\$ 538 \\
\$ 101 \\
\$ 269 \\
\$ 168\end{array}$ & $\begin{array}{r}.439 \\
.134 \\
.090 \\
.048\end{array}$ & $\begin{array}{l}-.435 \\
-.273 \\
-.102 \\
.564\end{array}$ \\
\hline
\end{tabular}

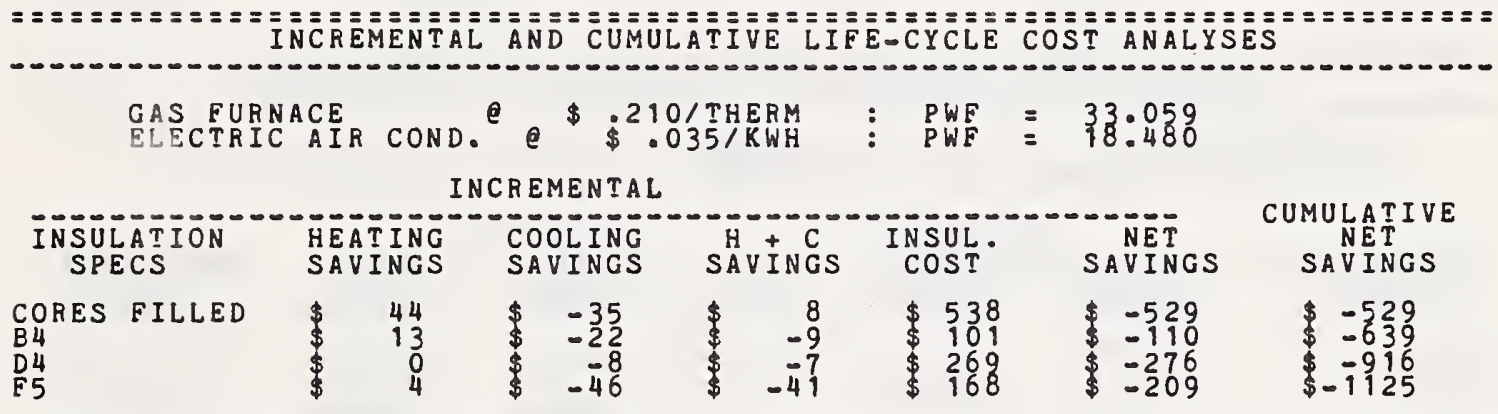

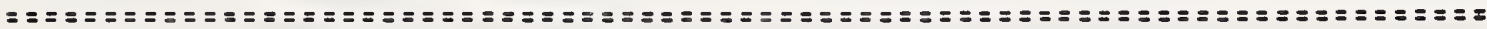

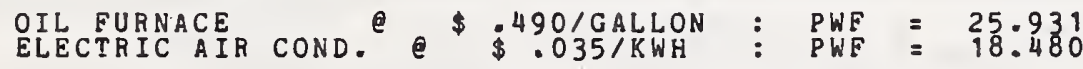

INCREMENTAL

\begin{tabular}{|c|c|c|c|c|c|c|}
\hline $\begin{array}{l}\text { INSULATION } \\
\text { SPECS }\end{array}$ & $\begin{array}{l}\text { HEATING } \\
\text { SAVINGS }\end{array}$ & $\begin{array}{l}\text { COOLING } \\
\text { SAVINGS }\end{array}$ & SÄVİNGS & $\begin{array}{l}\text { INSUL. } \\
\text { COST }\end{array}$ & SAVET INGS & $\begin{array}{l}\text { CUMULATIVE } \\
\text { SAVT INGS }\end{array}$ \\
\hline $\begin{array}{l}4 \\
4 \\
5\end{array}$ & $\begin{array}{r}51 \\
15 \\
1 \\
5\end{array}$ & $\begin{array}{l}\$-35 \\
\$-22 \\
\$-46\end{array}$ & $\begin{array}{r}15 \\
\$-6 \\
\$-7 \\
\$-40\end{array}$ & $\begin{array}{l}\$ 538 \\
\$ 101 \\
\$ 269 \\
\$ 168\end{array}$ & $\begin{array}{l}\$-522 \\
\$-107 \\
-276 \\
-208\end{array}$ & $\begin{array}{r}-522 \\
-530 \\
=-986 \\
-1115\end{array}$ \\
\hline
\end{tabular}

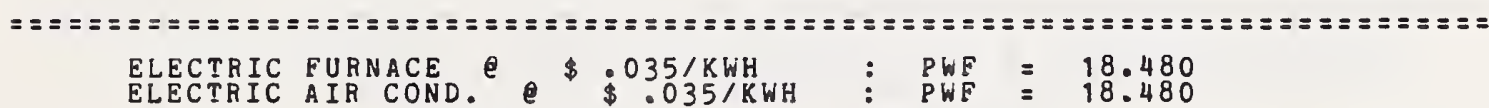

INCREMENTAL

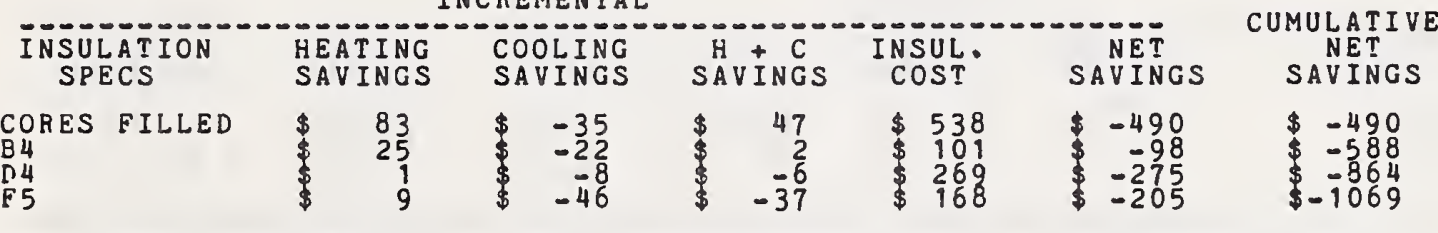

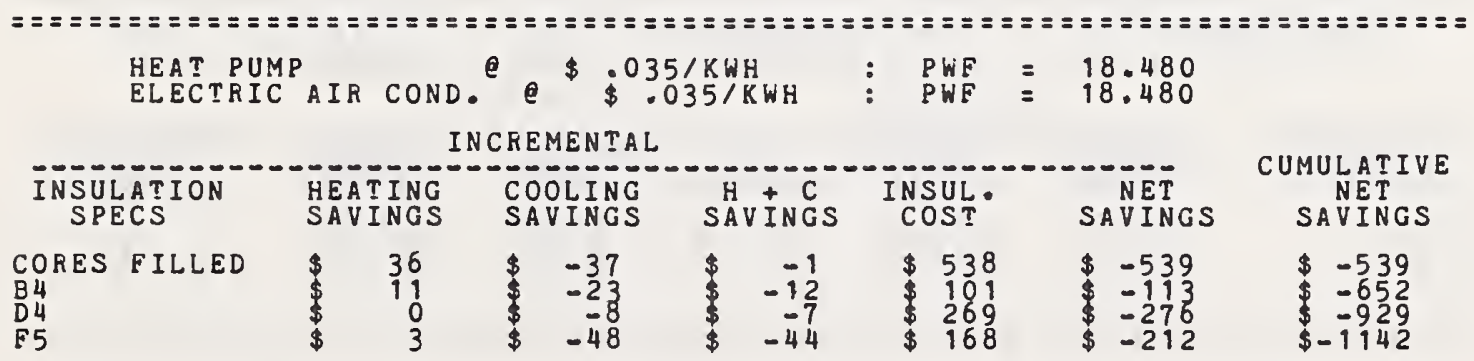

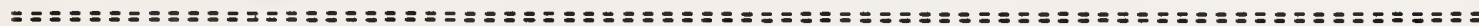


HUD 5

JACKSONVILLE FLORIDA 200 SQ FT HOUSE WOOD FRAME WALL COOLING LOADS BASED ON WINDOWS CLOSED

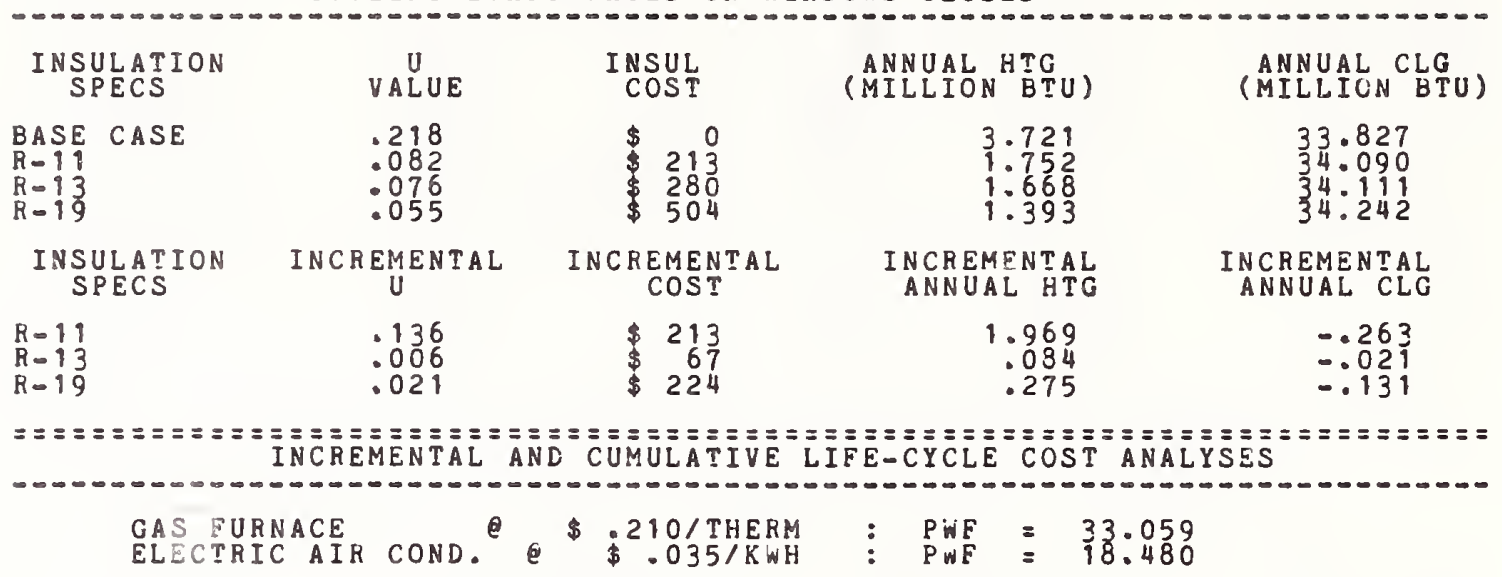
INCREMENTAL

\begin{tabular}{|c|c|c|c|c|c|c|}
\hline $\begin{array}{l}\text { INSULATION } \\
\text { SPECS }\end{array}$ & $\begin{array}{l}\text { HEATING } \\
\text { SAVINGS }\end{array}$ & $\begin{array}{l}\text { COOLING } \\
\text { SAVINGS }\end{array}$ & $\begin{array}{l}\text { H } \\
\text { SAVINGS }\end{array}$ & $\begin{array}{l}\text { INSUL. } \\
\text { COST }\end{array}$ & $\begin{array}{l}\text { NET } \\
\text { SAVINGS }\end{array}$ & $\begin{array}{l}\text { NET } \\
\text { SAVINGS }\end{array}$ \\
\hline $\begin{array}{l}R-11 \\
R-13 \\
R=19\end{array}$ & $\begin{array}{r}198 \\
8 \\
27\end{array}$ & $\begin{array}{l}-29 \\
-19\end{array}$ & $\begin{array}{r}176 \\
6 \\
16\end{array}$ & $\begin{array}{r}213 \\
\$ \quad 224\end{array}$ & $\begin{array}{r}-36 \\
-60 \\
-207\end{array}$ & $\begin{array}{r}\$-36 \\
\$-36 \\
\$-303\end{array}$ \\
\hline
\end{tabular}

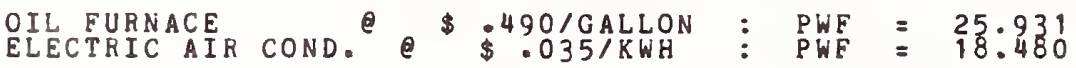

I NCREMENTAL

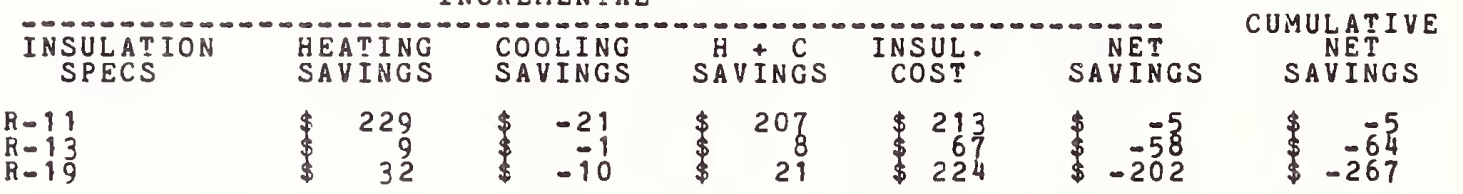

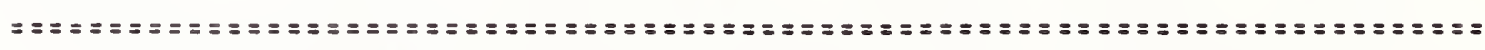

ELECTRIC FURNACE
ELECTRIC AIR COND. $\$ .035 / K W H$ INCREMENTAL

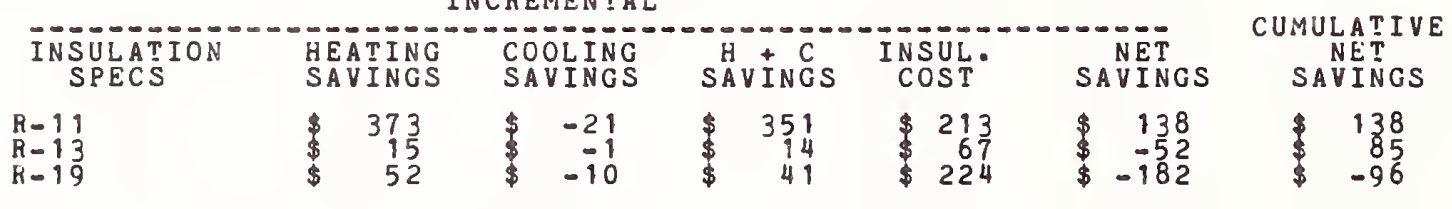

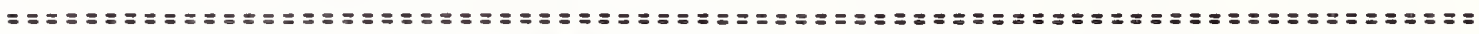

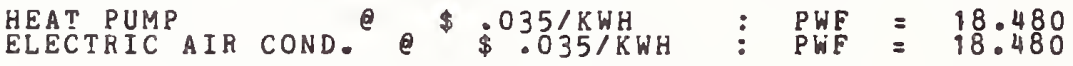

INCREMENTAL

\begin{tabular}{|c|c|c|c|c|c|c|}
\hline $\begin{array}{l}\text { NSULATION } \\
\text { SPECS }\end{array}$ & $\begin{array}{l}\text { HEATING } \\
\text { SAVINGS }\end{array}$ & $\begin{array}{l}\text { COOLING } \\
\text { SAVINGS }\end{array}$ & $\stackrel{H}{\text { SAVINGS }}+\underset{C}{C}$ & $\begin{array}{l}\text { INSUL: } \\
\text { COST }\end{array}$ & SAVINGS & $\begin{array}{c}\text { CUMULATIVE } \\
\text { NET } \\
\text { SAVINGS }\end{array}$ \\
\hline 13 & $\begin{array}{r}162 \\
6 \\
22\end{array}$ & $\begin{array}{l}-22 \\
-19\end{array}$ & $\begin{array}{r}139 \\
19\end{array}$ & $\begin{array}{r}213 \\
67 \\
224\end{array}$ & $\begin{array}{r}-73 \\
\$-212 \\
\$-212\end{array}$ & $\begin{array}{l}-173 \\
-135 \\
-347\end{array}$ \\
\hline
\end{tabular}

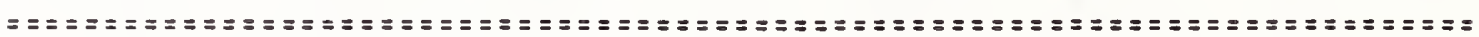


HUD 5

PHOENIX ARIZONA

1200 SO $R T$ HOUSE

WOOD FRAME WALL COOLING LOADS BASED ON WINDOWS CLOSED

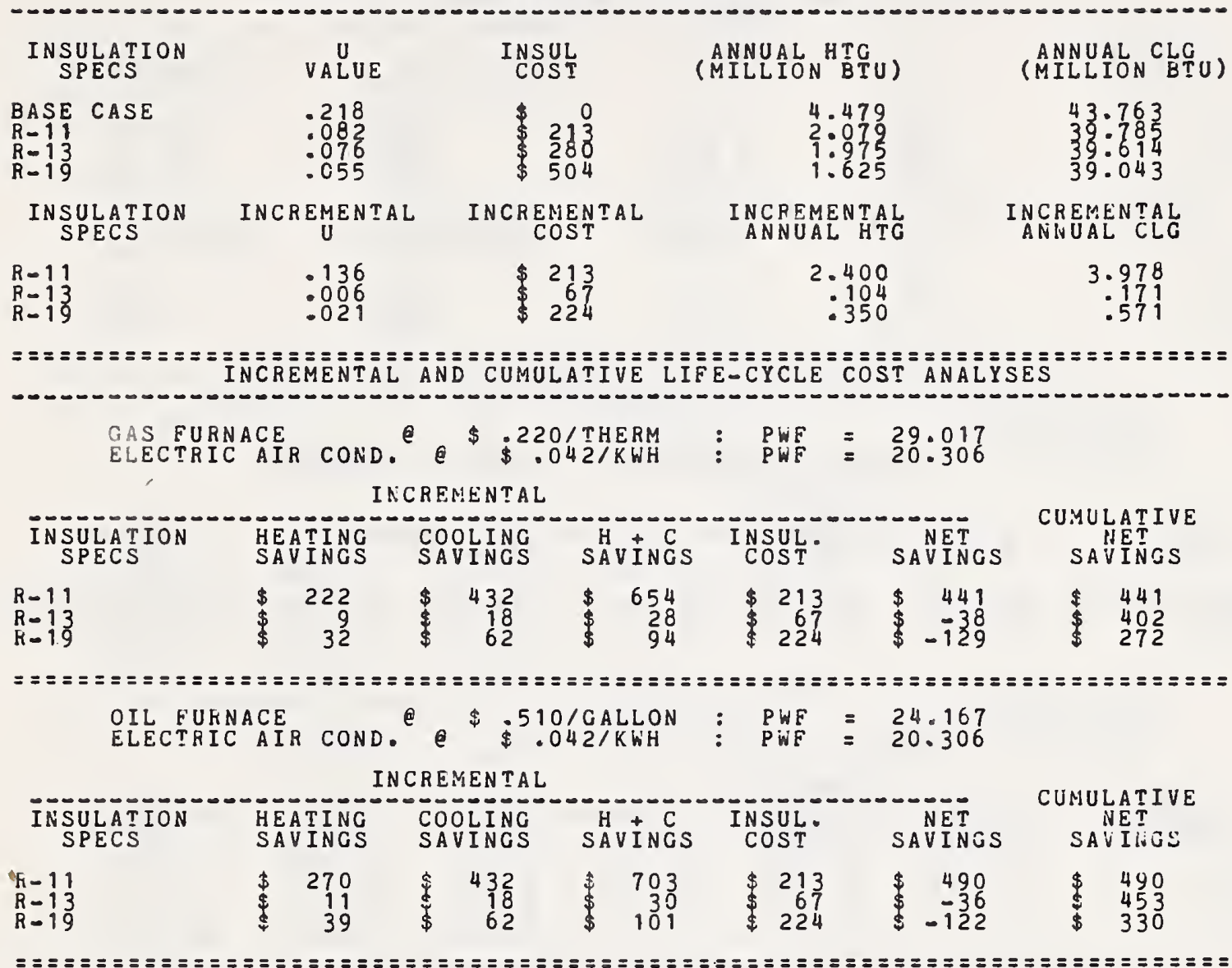

ELECTRIC FURNACE
ELECTRIC AIR COND. $\$ \$ 042 / K W H$
$\$$ PWF

INCREMENTAL

\begin{tabular}{|c|c|c|c|c|c|c|c|c|c|}
\hline $\begin{array}{l}\text { NSULATION } \\
\text { SPECS }\end{array}$ & $\begin{array}{l}\text { HEATTNG } \\
\text { SAVINOS }\end{array}$ & & INGS & SAU INGS & $\begin{array}{l}\text { INSUL } \\
\text { COST }\end{array}$ & & INGS & $\begin{array}{r}\text { CUilU } \\
\text { SA }\end{array}$ & $\begin{array}{l}\text { NET } \\
\text { VINGS }\end{array}$ \\
\hline 13 & $\begin{array}{r}599 \\
\$ \quad 25 \\
\$ \quad 87\end{array}$ & $\$$ & $\begin{array}{r}432 \\
18 \\
62\end{array}$ & $\begin{array}{r}1032 \\
\$ \quad 14 \\
\$ \quad 149\end{array}$ & $\begin{array}{r}213 \\
\$ \quad 274 \\
\$ 24\end{array}$ & $\$$ & $\begin{array}{l}819 \\
-22 \\
-74\end{array}$ & $\$$ & $\begin{array}{l}819 \\
796 \\
722\end{array}$ \\
\hline
\end{tabular}

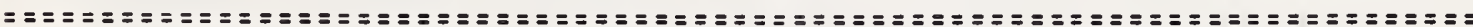

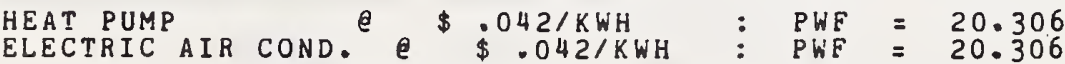

INCREMENTAL

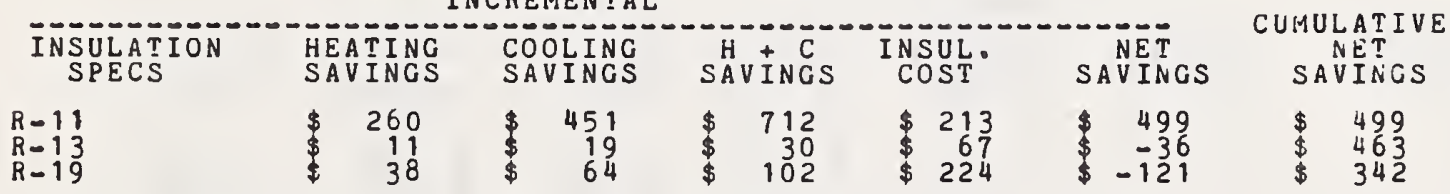

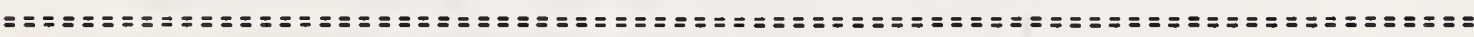


HUD 5

TAMPA ELORIDA 200 SO FT HOUSE WOOD FRAME WALL COOLING LOADS BASED ON WINDOWS CLOSED

\begin{tabular}{|c|c|c|c|c|}
\hline $\begin{array}{l}\text { INSULATION } \\
\text { SPECS }\end{array}$ & UALUE & $\begin{array}{l}\text { INSUL } \\
\text { COST }\end{array}$ & $\begin{array}{l}\text { ANNUAL HTG } \\
\text { (MILLION BTU) }\end{array}$ & $\begin{array}{l}\text { ANNUAL CLG } \\
\text { (MILLION BTU) }\end{array}$ \\
\hline $\begin{array}{l}B A S E \quad C A S E \\
K=19 \\
R=13 \\
K=19\end{array}$ & $\begin{array}{l}.218 \\
.082 \\
.076 \\
.055\end{array}$ & $\begin{array}{ll} & 210 \\
\$ & 213 \\
280 & 204\end{array}$ & $\begin{array}{r}.882 \\
.349 \\
.326 \\
.244\end{array}$ & $\begin{array}{l}39.801 \\
39: 809 \\
39.844 \\
39.969\end{array}$ \\
\hline $\begin{array}{l}\text { INSULATION } \\
\text { SPECS }\end{array}$ & INCREMENTAL & $\begin{array}{l}\text { INCREMENTAL } \\
\text { COST }\end{array}$ & $\begin{array}{l}\text { INCREMENTAL } \\
\text { ANNUAL HTG }\end{array}$ & $\begin{array}{l}\text { INCKE EIELTAL } \\
\text { ANISUAL CLG }\end{array}$ \\
\hline $\begin{array}{l}R=11 \\
R=13 \\
R=19\end{array}$ & $\begin{array}{l}.136 \\
.006 \\
.021\end{array}$ & $\begin{array}{r}213 \\
\$ \quad 67 \\
\$ 224\end{array}$ & $\begin{array}{l}.532 \\
.023 \\
.082\end{array}$ & $\begin{array}{l}-.808 \\
-.036 \\
-.125\end{array}$ \\
\hline \multicolumn{5}{|c|}{ 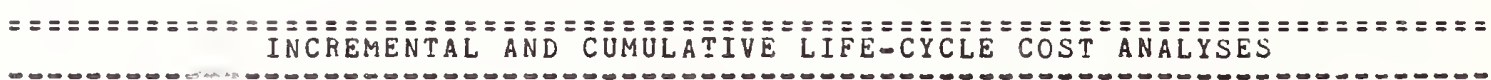 } \\
\hline
\end{tabular}

INCREMENTAL

\begin{tabular}{|c|c|c|c|c|c|c|}
\hline $\begin{array}{l}\text { INSULATION } \\
\text { SPECS }\end{array}$ & $\begin{array}{l}\text { HEATING } \\
\text { SAVINGS }\end{array}$ & $\begin{array}{l}\text { COOLING } \\
\text { SAVINGS }\end{array}$ & SA $\stackrel{H}{I} \stackrel{C}{\text { C }}$ & $\begin{array}{l}\text { INSUL. } \\
\text { COST }\end{array}$ & $\begin{array}{l}\text { SET } \\
\text { SAINGS }\end{array}$ & $\begin{array}{r}\text { CUMULATIVE } \\
\text { SAVIETES }\end{array}$ \\
\hline $\begin{aligned}-11 \\
-13 \\
-13\end{aligned}$ & $\begin{array}{r}53 \\
3 \\
8\end{array}$ & $\begin{array}{r}-66 \\
-2 \\
-10\end{array}$ & $\begin{array}{rr} & -12 \\
\$ & 0 \\
\$ & -2\end{array}$ & $\begin{array}{r}213 \\
\$ 274 \\
\$ 224\end{array}$ & $\begin{array}{r}-225 \\
\$-67 \\
-226\end{array}$ & $\begin{array}{l}\$-225 \\
\$-293 \\
\$-519\end{array}$ \\
\hline
\end{tabular}

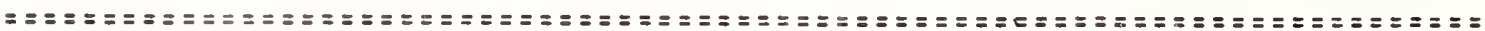

OIL FURNACE
ELECTRICAIH COND. $\$ 490 / G A L L O N: P W F=25.931$

INCREMENTAL

\begin{tabular}{|c|c|c|c|c|c|c|c|c|}
\hline $\begin{array}{l}\text { INSULATION } \\
\text { SPECS }\end{array}$ & & $\begin{array}{l}\text { IING } \\
\text { INGS }\end{array}$ & & $\begin{array}{l}\text { LING } \\
\text { INGS }\end{array}$ & SÄVİNGS & INSUL. & SAVET & $\begin{array}{c}\text { CORULATIVE } \\
\text { SAVITES }\end{array}$ \\
\hline $\begin{array}{l}R=11 \\
R=13 \\
R=19\end{array}$ & $\begin{array}{l}\$ \\
\$\end{array}$ & $\begin{array}{r}61 \\
2 \\
9\end{array}$ & $\$$ & $\begin{array}{l}-56 \\
-2 \\
-10\end{array}$ & $\begin{array}{r}-4 \\
0 \\
0\end{array}$ & $\begin{array}{r}213 \\
\$ 27 \\
\$ 224\end{array}$ & $\begin{array}{rr} & -217 \\
\$ & -67 \\
\$ & -224\end{array}$ & $\begin{array}{l}\$-217 \\
\$-284 \\
-509\end{array}$ \\
\hline
\end{tabular}

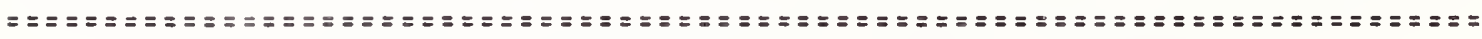

ELECTRIC FURNACE e $\$ .035 / \mathrm{KWH}$
ELECTRIC AIR COND. $\$$ PWF $=18.480$

INCREMENTAL

\begin{tabular}{|c|c|c|c|c|c|c|}
\hline $\begin{array}{l}\text { INSULATION } \\
\text { SPECS }\end{array}$ & $\begin{array}{l}\text { HEATING } \\
\text { SAVINGS }\end{array}$ & $\begin{array}{l}\text { COOLING } \\
\text { SAVINGS }\end{array}$ & $\begin{array}{l}\text { SAVINGS } \\
\text { SAV }\end{array}$ & INSUL. & SAVINGS & $\begin{array}{l}\text { CUMULATIVE } \\
\text { NETT } \\
\text { SAVINCS }\end{array}$ \\
\hline $\begin{array}{l}-1 \\
-1\end{array}$ & $\begin{array}{r}100 \\
4 \\
15\end{array}$ & $\begin{array}{l}-66 \\
-2 \\
-10\end{array}$ & $\begin{array}{r}34 \\
5 \\
5\end{array}$ & $\begin{array}{r}213 \\
\$ 27 \\
224\end{array}$ & $\begin{array}{r}-178 \\
\$-65 \\
-218\end{array}$ & $\begin{array}{l}\$-178 \\
\$-244 \\
-462\end{array}$ \\
\hline
\end{tabular}

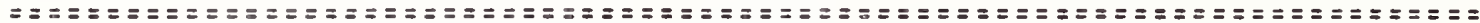
HEATPUMP
ELECTRICAIR COND. $\$$ \$ $\$ 35 / K W H$ INCREMENTAL

\begin{tabular}{|c|c|c|c|c|c|c|}
\hline $\begin{array}{l}\text { INSULATION } \\
\text { SPECS }\end{array}$ & $\begin{array}{l}\text { HEAT ING } \\
\text { SAVINGS }\end{array}$ & $\begin{array}{l}\text { COOLING } \\
\text { SAVINGS }\end{array}$ & SAVINGS & $\begin{array}{l}\text { INSUL. } \\
\text { COST }\end{array}$ & $\begin{array}{l}\text { NET } \\
\text { SAVINGS }\end{array}$ & $\begin{array}{l}\text { CUMULATIVE } \\
\text { NET TANGS }\end{array}$ \\
\hline $\begin{array}{r}11 \\
-13 \\
-19\end{array}$ & $\begin{array}{r}43 \\
1 \\
6\end{array}$ & $\begin{array}{r}\quad-69 \\
\$ \quad-10\end{array}$ & $\begin{array}{r}-25 \\
-1 \\
-3\end{array}$ & $\begin{array}{r}\$ 213 \\
\$ 27 \\
\$ 224\end{array}$ & $\begin{array}{r}\$-238 \\
\$-68 \\
\$-227\end{array}$ & $\begin{array}{l}\$-238 \\
\$-306 \\
\$-534\end{array}$ \\
\hline
\end{tabular}

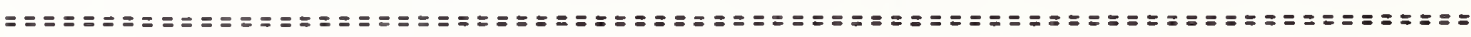




\section{APPENDIX C \\ Computer Printouts of Economic Analyses \\ for Tampa, Phoenix and Jacksonville --}

Cooling Savings Based on Cooling Above $78^{\circ} \mathrm{F}\left(25.5^{\circ} \mathrm{C}\right)$ Outdoor Temperature 
HUD 1

JACKSONVILLE FLORIDA

8 IN BLOCK (OPEN CORE) COOLING LOADS BASED ON COOLING ABOVE 78 F OUTSIDE

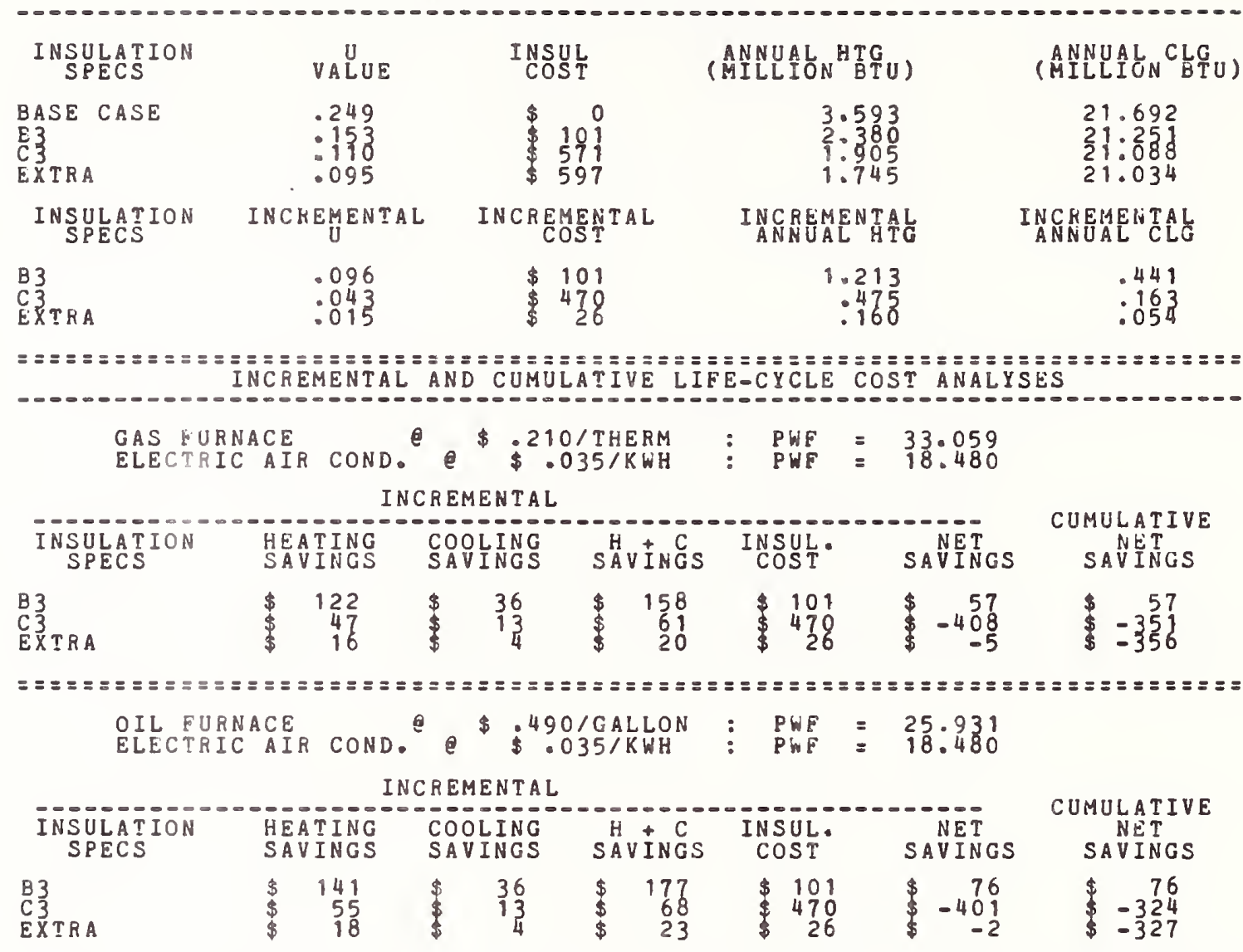

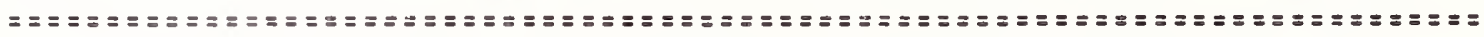
ELECTRIC FURNACE e $\$ \$ 035 / K W H$
ELECTRIC AIR COND. $\$ \$$ PWF $=18.480$ INCREMENTAL

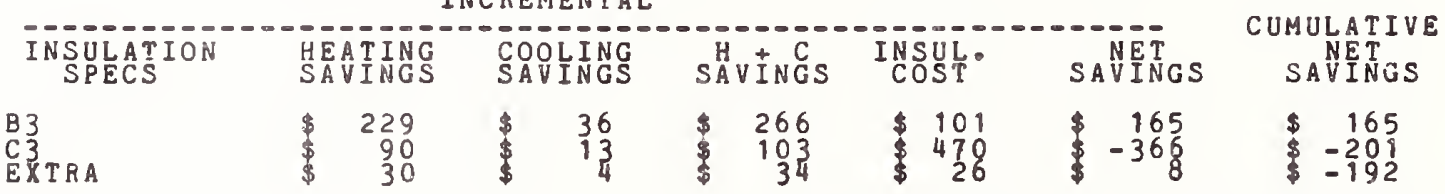

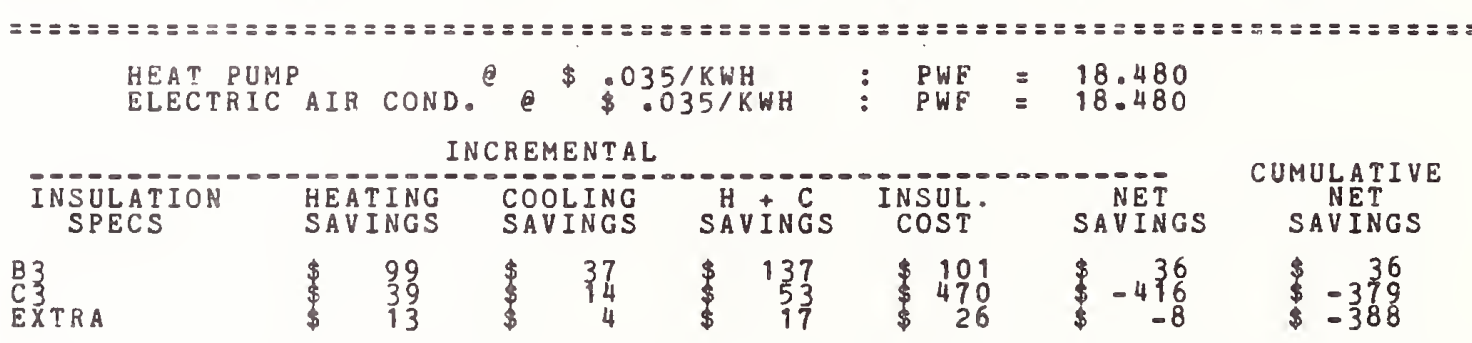

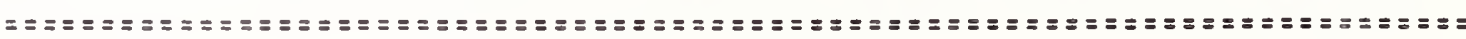


HUD 1

PHOENIX ARIZONA 200 SO FT HOUSE IN BLOCK (OPEN CORE) COOLING LOADS BASED ON COOLING ABOVE 78 F OUTSIDE

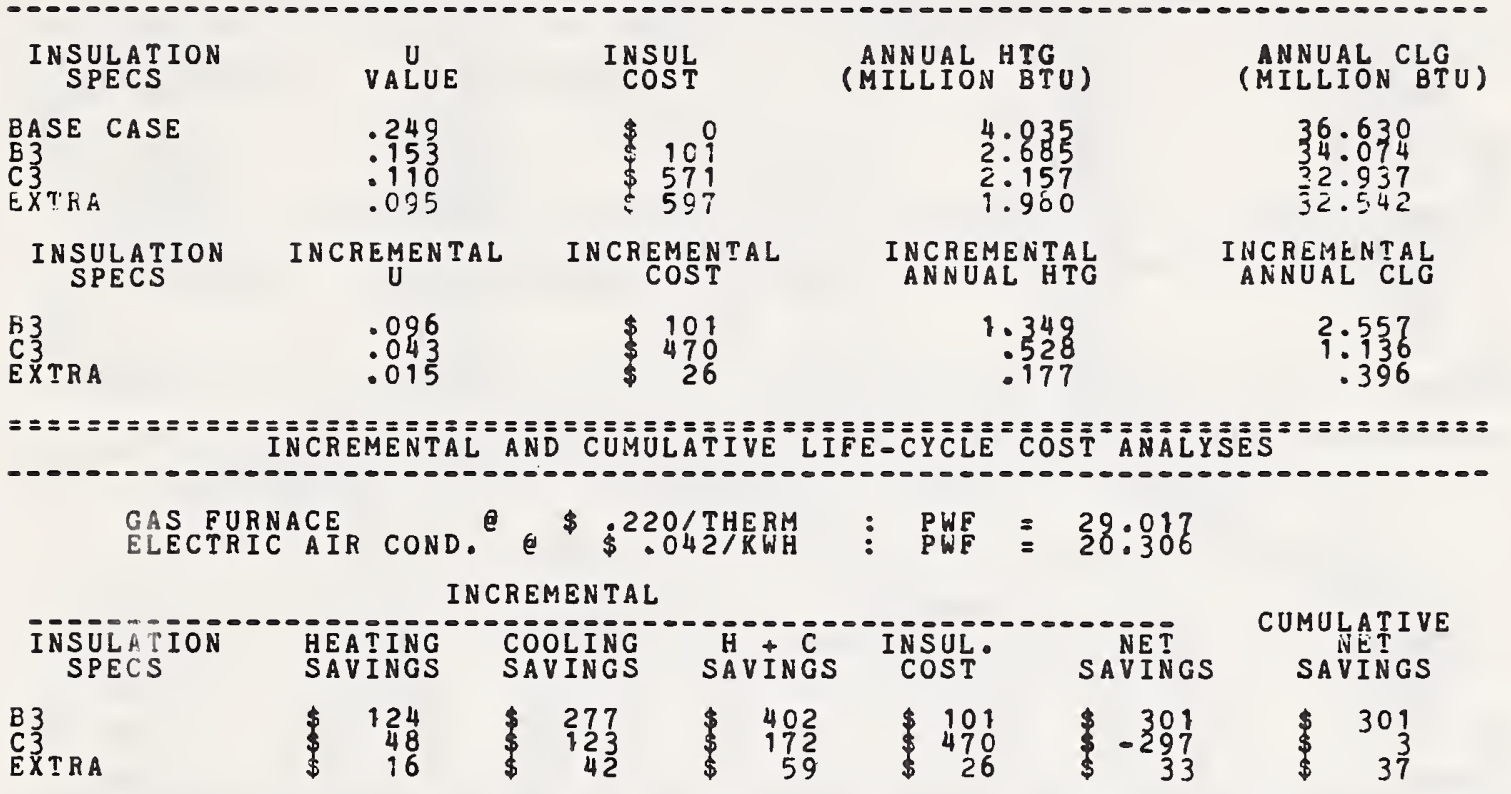

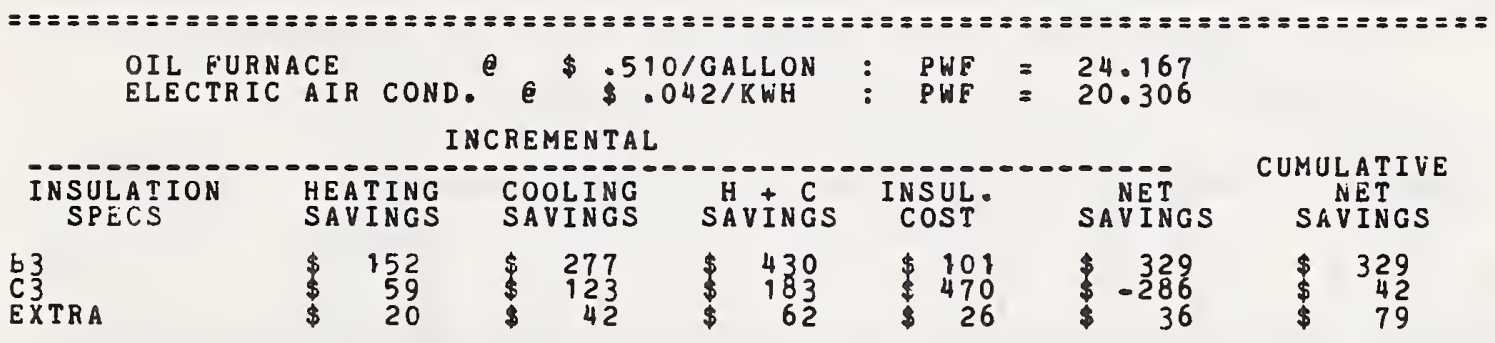

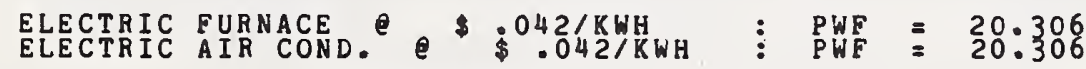

INCREMENTAL

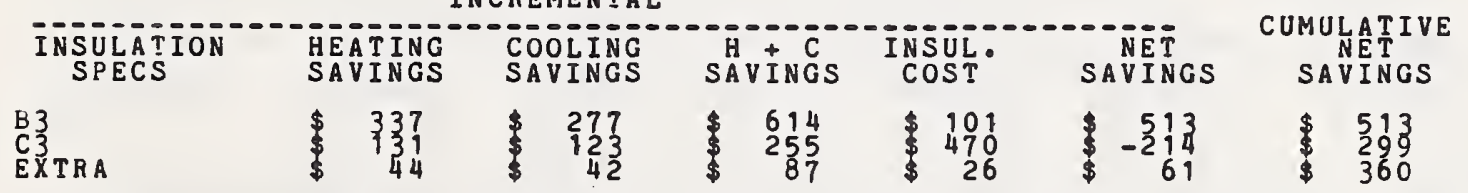

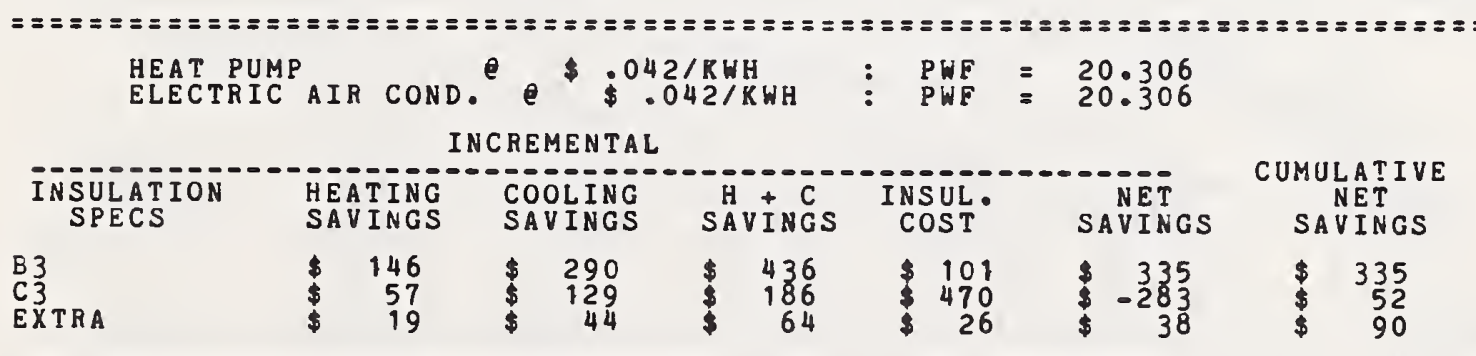


HUD 1

TAMPA FLOAIDA 8 IN BLOCK (OPEN CORE) COOLING LOADS BASED ON COOLING ABOVE 78 F OUTSIDE

\begin{tabular}{|c|c|c|c|c|}
\hline $\begin{array}{l}\text { INSULATION } \\
\text { SPECS }\end{array}$ & VALUE & $\begin{array}{l}\text { INSUL } \\
\text { COST }\end{array}$ & (MNLUAL HTG & (MNNUAL CLG \\
\hline $\begin{array}{l}\text { BASE CASE } \\
\text { B } \\
\text { C } 3 \\
\text { EXTRA }\end{array}$ & $\begin{array}{r}.249 \\
.153 \\
.110 \\
.095\end{array}$ & $\begin{array}{lr}\$ & 0 \\
\$ & 101 \\
\$ & 579 \\
\$ & 597\end{array}$ & $\begin{array}{r}.850 \\
.505 \\
.383 \\
.343\end{array}$ & $\begin{array}{l}24.292 \\
23.831 \\
23.661 \\
23.606\end{array}$ \\
\hline $\begin{array}{l}\text { INSULAT ION } \\
\text { SPECS }\end{array}$ & INCREMENTAL & $\begin{array}{c}\text { INCREMENTAL } \\
\text { COST }\end{array}$ & $\begin{array}{l}\text { INCREMENTAL } \\
\text { ANNUAL HTG }\end{array}$ & $\begin{array}{l}\text { INCREMENTAL } \\
\text { ANNUAL CLU }\end{array}$ \\
\hline $\begin{array}{l}\text { B3 } \\
C 33 \\
\text { EXTRA }\end{array}$ & $\begin{array}{l}.096 \\
.043 \\
.015\end{array}$ & $\begin{array}{r}\$ 109 \\
\$ 470 \\
\$ 26\end{array}$ & $\begin{array}{r}.345 \\
: 122 \\
.040\end{array}$ & $\begin{array}{l}.461 \\
.169 \\
.056\end{array}$ \\
\hline
\end{tabular}

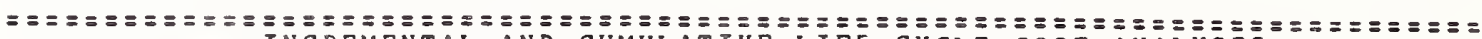
INCREMENTAL AND CUMULATIVE LIFE-CYCLE COST ANALYSES

GAS PURNACE
ELECTRICAIR COND. e $e^{\$ 210 / T H E R M ~} \$$ PWE INCREMENTAL

\begin{tabular}{|c|c|c|c|c|c|c|}
\hline $\begin{array}{l}\text { IN SUL AT ION } \\
\text { SPECS }\end{array}$ & $\begin{array}{l}\text { HEATING } \\
\text { SAVINGS }\end{array}$ & $\begin{array}{l}\text { COOLING } \\
\text { SAVINGS }\end{array}$ & SÄU İNGS & INSUL. & SAVET & $\begin{array}{c}\text { COMULATIVE } \\
\text { NET } \\
\text { SAVINGS }\end{array}$ \\
\hline$\left\{\begin{array}{l}3 \\
3 \\
x T A\end{array}\right.$ & $\begin{array}{l}34 \\
12 \\
4\end{array}$ & $\begin{array}{l}38 \\
13 \\
4\end{array}$ & $\begin{array}{r}72 \\
26 \\
8\end{array}$ & $\begin{array}{r}101 \\
\$ 470 \\
\$ \quad 26\end{array}$ & $\begin{array}{r}-28 \\
\$-443 \\
-17\end{array}$ & $\begin{aligned} & -28 \\
& -472 \\
& -489\end{aligned}$ \\
\hline
\end{tabular}

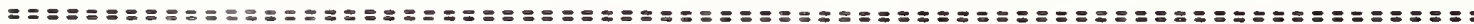

OIL FURNACE
ELECTRICAIR COND. e $e^{400 / G A L L O N}:$ PWF $=25.931$

INCREMENTAL

\begin{tabular}{|c|c|c|c|c|c|c|}
\hline $\begin{array}{l}\text { IN SULATION } \\
\text { SPECS }\end{array}$ & $\begin{array}{l}\text { HEATING } \\
\text { SAVINGS }\end{array}$ & $\begin{array}{l}\text { COOLING } \\
\text { SAVINGS }\end{array}$ & $\begin{array}{l}H+\underset{C}{C} \\
\text { SAVINGS }\end{array}$ & $\begin{array}{l}\text { INSUL. } \\
\text { COST }\end{array}$ & $\begin{array}{c}\text { NET } \\
\text { SAVINGS }\end{array}$ & $\begin{array}{c}\text { CUMULATIVE } \\
\text { NETTES } \\
\text { SAVINGS }\end{array}$ \\
\hline KRA & $\begin{array}{r}40 \\
14 \\
4\end{array}$ & $\begin{array}{l}38 \\
13 \\
4\end{array}$ & $\begin{array}{r}78 \\
28 \\
9\end{array}$ & $\begin{array}{r}101 \\
\$ \quad 470 \\
\$ \quad 26\end{array}$ & $\begin{array}{r}\$-22 \\
\$-441 \\
\$-16\end{array}$ & $\begin{array}{l}\$-22 \\
\$-464 \\
\$-481\end{array}$ \\
\hline
\end{tabular}

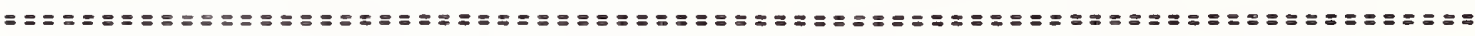

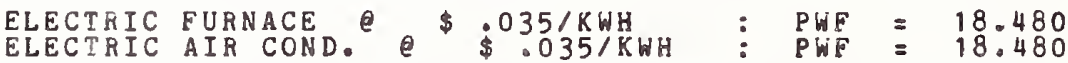
INCREMENTAL

\begin{tabular}{|c|c|c|c|c|c|c|}
\hline $\begin{array}{l}\text { IN SULAT ION } \\
\text { SPECS }\end{array}$ & $\begin{array}{l}\text { HEATING } \\
\text { SAVINGS }\end{array}$ & $\begin{array}{l}\text { COOLING } \\
\text { SAVINGS }\end{array}$ & SAVINGS & INSULT. & SAVINGS & SAVINGS \\
\hline $\begin{array}{l}3 \\
3 \\
\text { XTRA }\end{array}$ & $\begin{array}{l}65 \\
23\end{array}$ & $\begin{array}{l}38 \\
13 \\
4\end{array}$ & $\begin{array}{r}103 \\
\$ \quad 37\end{array}$ & $\begin{array}{r}101 \\
470 \\
426\end{array}$ & $\begin{array}{l}\$-432 \\
\$-13\end{array}$ & $\begin{array}{l}\$ \\
\$-430 \\
-444\end{array}$ \\
\hline
\end{tabular}

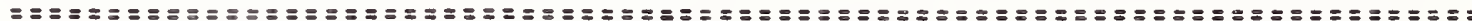

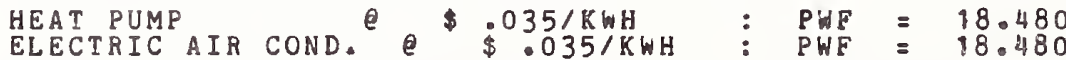
INCREMENTAL

\begin{tabular}{|c|c|c|c|c|c|c|}
\hline $\begin{array}{l}\text { NSULATION } \\
\text { SPECS }\end{array}$ & $\begin{array}{l}\text { HEATING } \\
\text { SAVINGS }\end{array}$ & $\begin{array}{l}\text { COOLING } \\
\text { SAVINGS }\end{array}$ & $\stackrel{H}{\text { SAVINAGS }}$ & $\begin{array}{l}\text { INSUL. } \\
\text { COST }\end{array}$ & SAVET & $\begin{array}{c}\text { NET } \\
\text { SAVINGS }\end{array}$ \\
\hline RA & $\begin{array}{ll}\$ & 28 \\
\$ & 10 \\
\$ & 3\end{array}$ & $\begin{array}{r}39 \\
14 \\
4\end{array}$ & $\begin{array}{r}68 \\
24 \\
8\end{array}$ & $\begin{array}{r}\$ 101 \\
\$ 470 \\
\$ \quad 26\end{array}$ & $\begin{array}{r}-32 \\
-445 \\
-17\end{array}$ & $\begin{array}{ll} & -32 \\
\$ & -478 \\
& -496\end{array}$ \\
\hline
\end{tabular}


HUD 2 JACKSONVILLE FLORIDA 1200 SQ FT HOUSE 8 IN BLOCK (OPEN CORE) COOLING LOADS BASED ON COOLING ABOVE 78 F OUTSIDE

\begin{tabular}{|c|c|c|c|c|}
\hline $\begin{array}{l}\text { INSULATION } \\
\text { SPECS }\end{array}$ & $\stackrel{U}{\text { VALUE }}$ & $\begin{array}{l}\text { INSUL } \\
\text { COST }\end{array}$ & $\begin{array}{l}\text { ANNUAL HTG } \\
\text { (MILLION BTU) }\end{array}$ & $\begin{array}{l}\text { ANNUAL CLG } \\
\text { (MILLION BTU) }\end{array}$ \\
\hline $\begin{array}{l}\text { BASE CASE } \\
\text { B3 } \\
\text { D3 } \\
\text { F2 }\end{array}$ & $\begin{array}{r}.249 \\
: 153 \\
.143 \\
.091\end{array}$ & $\begin{array}{r}0 \\
101 \\
378 \\
\$ 538\end{array}$ & $\begin{array}{l}3.593 \\
2.380 \\
2: 256 \\
1: 703\end{array}$ & $\begin{array}{l}21.692 \\
21.251 \\
21.206 \\
21.019\end{array}$ \\
\hline $\begin{array}{l}\text { INSULATION } \\
\text { SPECS }\end{array}$ & INCREMENTAL & $\begin{array}{c}\text { INCREMENTAL } \\
\text { COST }\end{array}$ & $\begin{array}{l}\text { INCREMENTAL } \\
\text { ANNUAL HTG }\end{array}$ & $\begin{array}{l}\text { INCREMENTAL } \\
\text { ANNUAL CLG }\end{array}$ \\
\hline $\begin{array}{l}\text { B } 3 \\
\text { D 3 } \\
\text { F2 }\end{array}$ & $\begin{array}{l}.096 \\
.010 \\
.052\end{array}$ & $\begin{array}{l}101 \\
\$ 269 \\
\$ 168\end{array}$ & $\begin{array}{r}1.213 \\
.124 \\
.553\end{array}$ & $\begin{array}{r}.441 \\
.045 \\
.187\end{array}$ \\
\hline
\end{tabular}

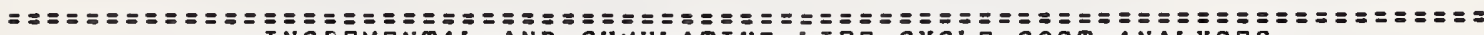
INCREMENTAL AND CUMULATIVE LIFE-CYCLE COST ANALYSES

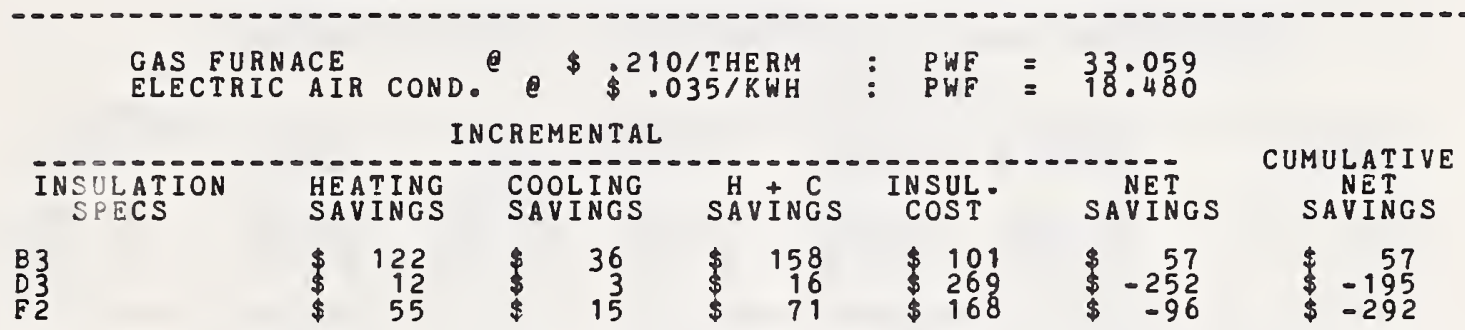

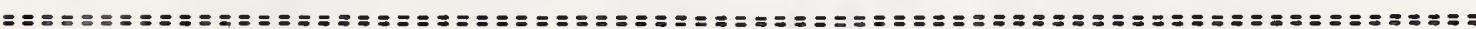

OILETRICACE

INCREMENTAL

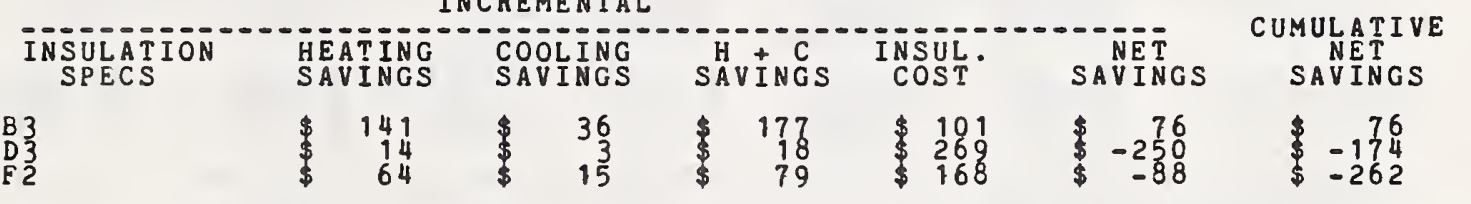

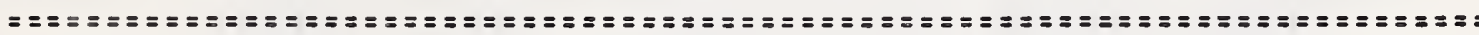
ELECTRIC FURNACE
ELECTRIC AIR COND. $\$ \$ 035 / K W H$
$\$ P W H F$ INCREMENTAL

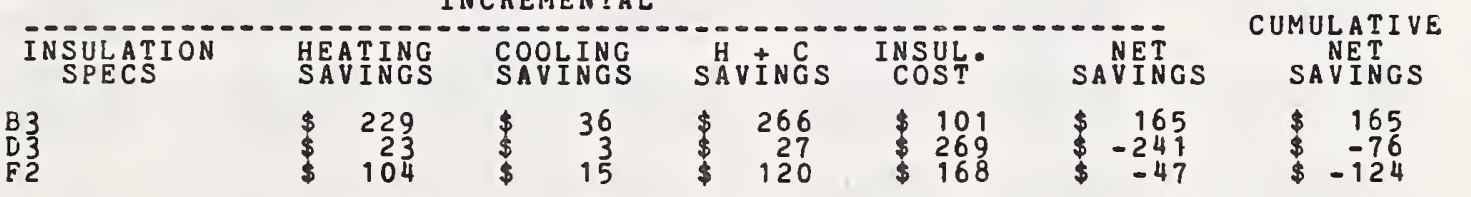

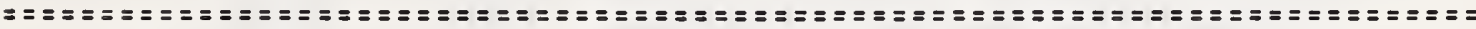
HEAT PUMP
ELECTRIC AIR COND. e $\$ \$ 035 / K W H$ INCREMENTAL

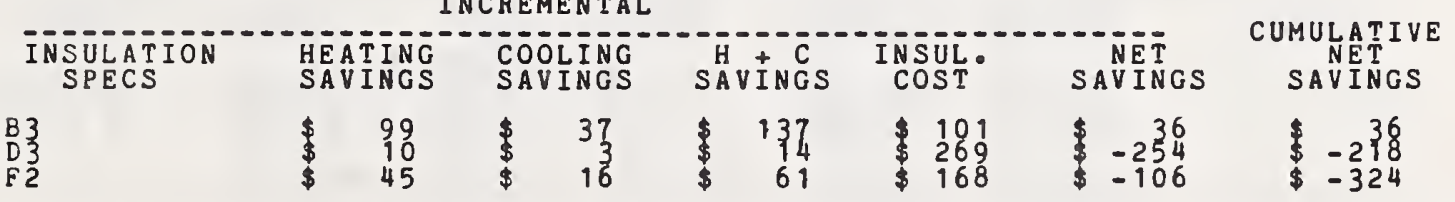


HUD 2

PHOENIX ARIZONA 1200 SO FT HOUSE IN BLOCK (OPEN CORE)

COOLING LOADS BASED ON COOLING ABOVE 78 F OUTSIDE

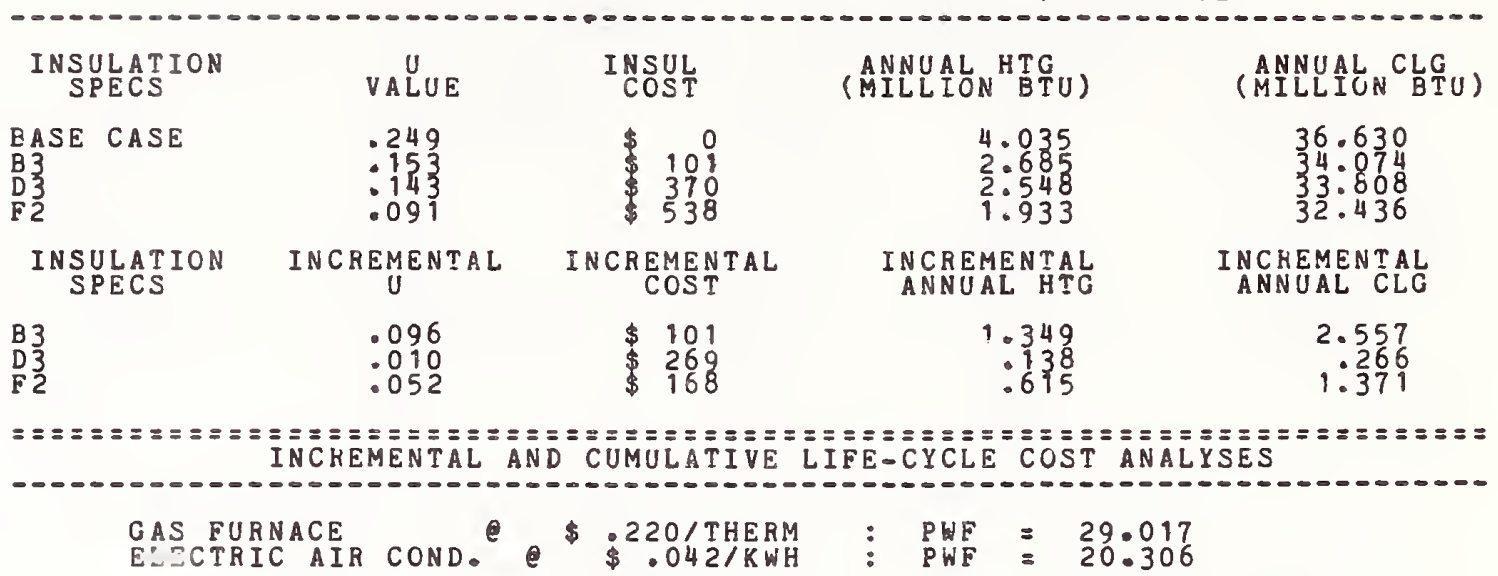

INCREMENTAL

\begin{tabular}{|c|c|c|c|c|c|c|c|}
\hline $\begin{array}{l}\text { INSULATION } \\
\text { SPECS }\end{array}$ & $\begin{array}{l}\text { HEAT ING } \\
\text { SAVINGS }\end{array}$ & $\begin{array}{l}\text { COOLING } \\
\text { SAVINGS }\end{array}$ & SAVINGS & $\begin{array}{l}\text { INSUL } \\
\text { COST }\end{array}$ & SAVINGS & $\begin{array}{r}\text { CUMO } \\
\text { SA }\end{array}$ & $\begin{array}{l}\text { LATIVE } \\
\text { NETT } \\
\text { VINGS }\end{array}$ \\
\hline 3 & $\begin{array}{r}124 \\
12 \\
\quad 56\end{array}$ & $\begin{array}{r}277 \\
288 \\
148\end{array}$ & $\begin{array}{l}402 \\
41 \\
205\end{array}$ & $\begin{array}{l}101 \\
\$ 269 \\
\$ 168\end{array}$ & $\begin{array}{rr} & 301 \\
\$ & -227 \\
\$ & 37\end{array}$ & $\$$ & $\begin{array}{l}301 \\
172\end{array}$ \\
\hline
\end{tabular}

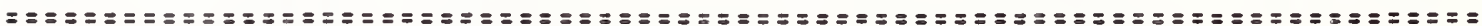

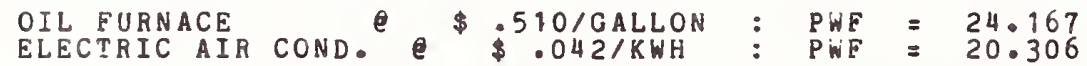

INCREMENTAL

\begin{tabular}{|c|c|c|c|c|c|c|}
\hline $\begin{array}{l}\text { INSULATION } \\
\text { SPECS }\end{array}$ & $\begin{array}{l}\text { HEATING } \\
\text { SAVINGS }\end{array}$ & $\begin{array}{l}\text { COOLING } \\
\text { SAVINGS }\end{array}$ & $\stackrel{H}{S A V I N G S}$ & $\begin{array}{l}\text { INSUL. } \\
\text { COST }\end{array}$ & $\begin{array}{l}\text { NET } \\
\text { SAVINGS }\end{array}$ & $\begin{array}{l}\text { UMULATIVE } \\
\text { NET } \\
\text { SAVINGS }\end{array}$ \\
\hline $\begin{array}{l}3 \\
3\end{array}$ & $\begin{array}{r}\$ \\
\$ \\
\$ \\
\$ \quad 69\end{array}$ & $\begin{array}{r}277 \\
28 \\
148\end{array}$ & $\begin{array}{r}430 \\
44 \\
218\end{array}$ & $\begin{array}{l}\$ 101 \\
\$ 269 \\
\$ 168\end{array}$ & $\begin{array}{rr}\$ & 329 \\
\$ & -224 \\
\$ & 50\end{array}$ & $\begin{array}{l}329 \\
104 \\
154\end{array}$ \\
\hline
\end{tabular}

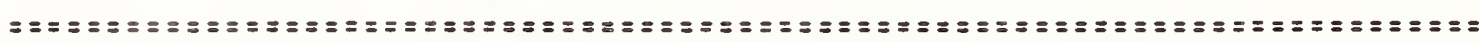
ELECTRIC FURNACE
ELECTRIC AIR COND。 $\$ \$ .042 / R W H$
PWF INCREMENTAL

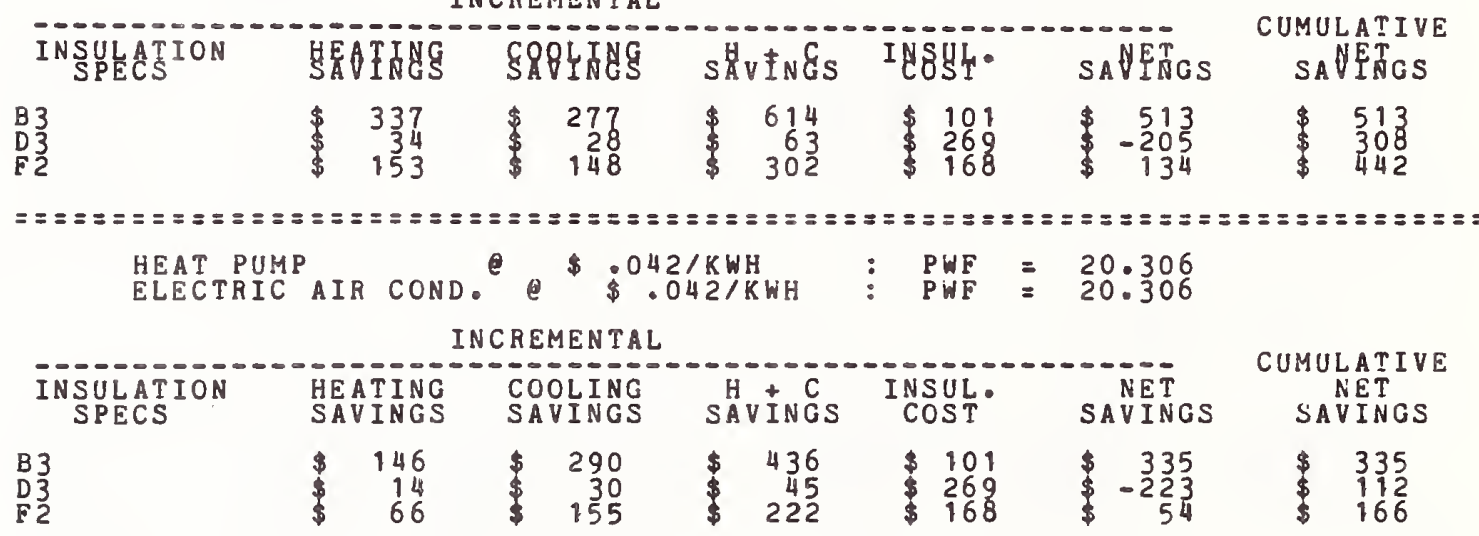

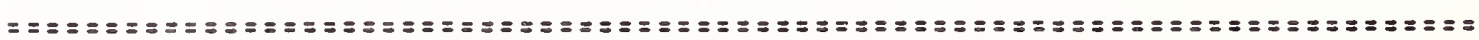


HUD2

TAMPA FLORIDA 1200 SQ FT HOUSE 8 IN BLOCK (OPEN CORE) COOLING LOADS BASED ON COOLING ABOVE 78 E OUTSIDE

\begin{tabular}{|c|c|c|c|c|}
\hline $\begin{array}{l}\text { INSULATION } \\
\text { SPECS }\end{array}$ & VALUE & $\begin{array}{l}\text { INSUL } \\
\text { COST }\end{array}$ & $\begin{array}{l}\text { ANNUAL } \\
\text { (MILLION }\end{array}$ & $\begin{array}{l}\text { ANNUAL CLG } \\
\text { (MILLION BTU) }\end{array}$ \\
\hline $\begin{array}{l}\text { BASE CASE } \\
\text { B3 } \\
\text { D } 3 \\
E^{2} 2\end{array}$ & $\begin{array}{l}.249 \\
.153 \\
.143 \\
.091\end{array}$ & $\begin{array}{rr}\$ & 0 \\
\$ & 101 \\
& 370 \\
\$ & 538\end{array}$ & $\begin{array}{r}.850 \\
.505 \\
.471 \\
.333\end{array}$ & $\begin{array}{l}24 \cdot 292 \\
23.831 \\
23.734 \\
23.591\end{array}$ \\
\hline $\begin{array}{l}\text { INSULATION } \\
\text { SPECS }\end{array}$ & INCREMENTAL & $\begin{array}{c}\text { INCREHENTAL } \\
\text { COST }\end{array}$ & $\begin{array}{l}\text { INCREMENTAL } \\
\text { ANNUAL HTG }\end{array}$ & $\begin{array}{l}\text { INCKEMENTAL } \\
\text { ANNUAL CLG }\end{array}$ \\
\hline $\begin{array}{l}\mathrm{B} 3 \\
\mathrm{D} 3 \\
82\end{array}$ & $\begin{array}{r}.096 \\
.010 \\
.052\end{array}$ & $\begin{array}{l}101 \\
\$ 269 \\
\$ 168\end{array}$ & $\begin{array}{r}.345 \\
: 035 \\
: 138\end{array}$ & $\begin{array}{r}.461 \\
.047 \\
.193\end{array}$ \\
\hline
\end{tabular}

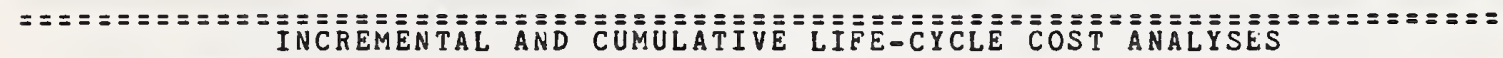

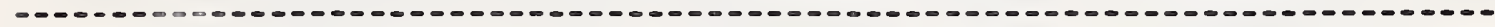

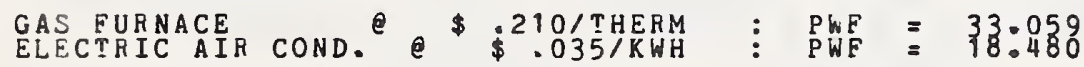

INCREMENTAL

\begin{tabular}{|c|c|c|c|c|c|c|c|c|}
\hline $\begin{array}{l}\text { INSULATION } \\
\text { SPECS }\end{array}$ & & INGS & & {$\left[\begin{array}{l}I N G \\
N G S\end{array}\right.$} & SÄVINGS & $\begin{array}{l}\text { INSUL. } \\
\text { COST }\end{array}$ & SAVINGS & $\begin{array}{l}\text { CUMULATIVE } \\
\text { NET } \\
\text { SAVINGS }\end{array}$ \\
\hline 3 & $\$$ & $\begin{array}{l}34 \\
3 \\
13\end{array}$ & $\$$ & $\begin{array}{l}38 \\
3 \\
15\end{array}$ & $\begin{array}{l}72 \\
7 \\
29\end{array}$ & $\begin{array}{l}\$ 101 \\
\$ 269 \\
\$ 168\end{array}$ & $\begin{array}{l}\$=-28 \\
\$=138\end{array}$ & $\begin{aligned} & -28 \\
& -289 \\
- & -428\end{aligned}$ \\
\hline
\end{tabular}

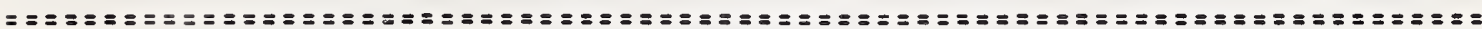

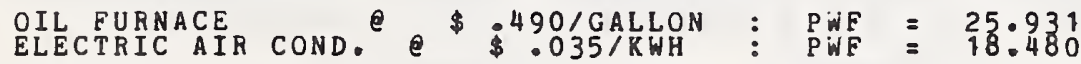

INCREMENTAL

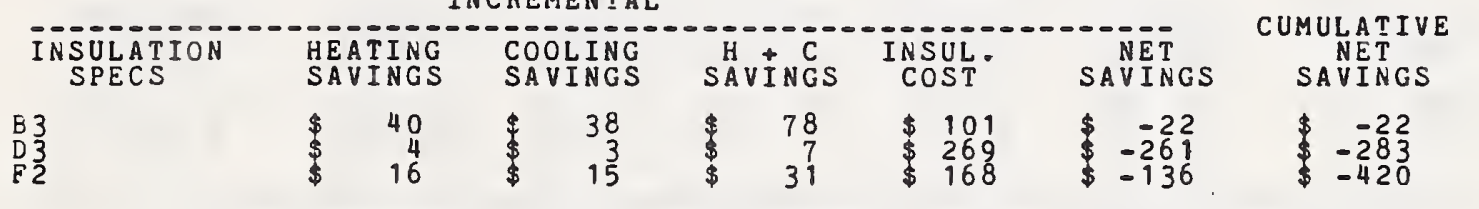

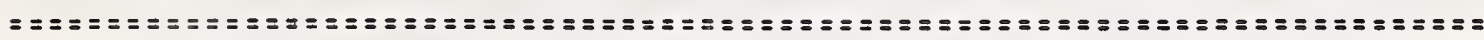

ELECTRIC FURNACE
ELECTRIC AIR COND. $\$ \$ 035 / K W H$
$\$ P H F$ INCREMENTAL

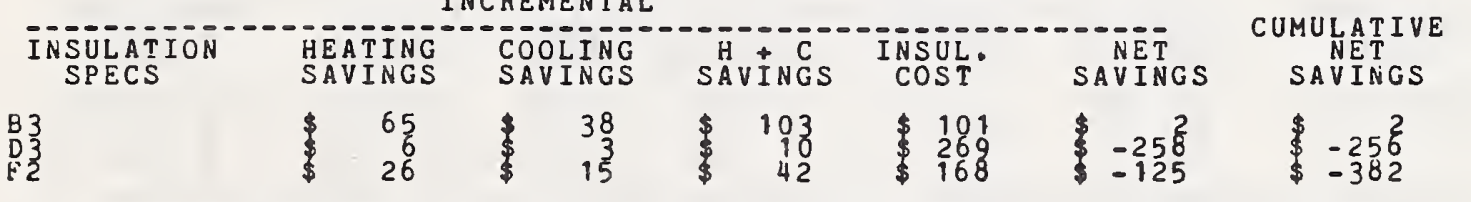

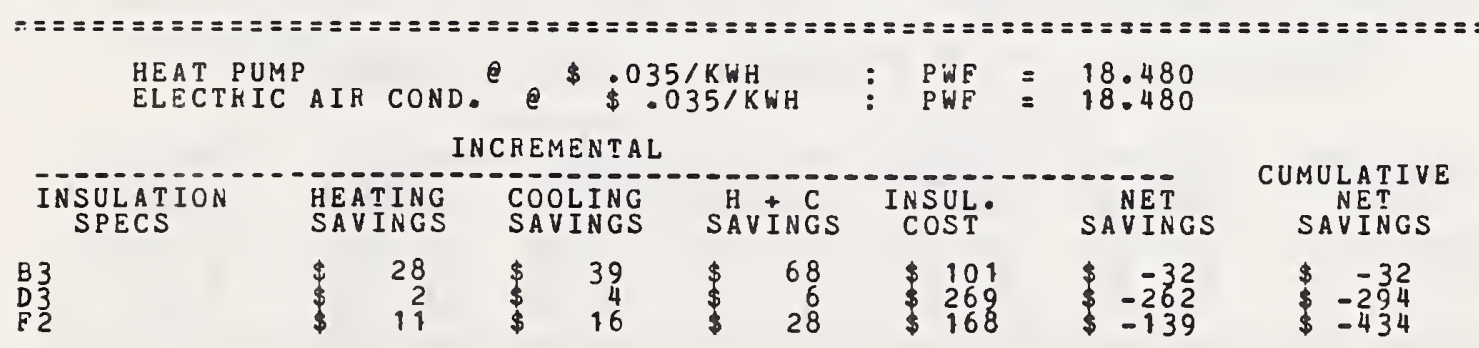

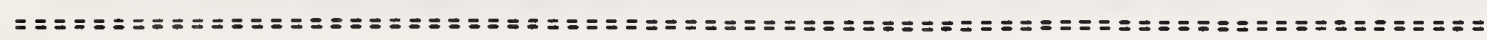


HUD 3

JACKSONVILLE FLORIDA $120^{\circ}$ SO FT HOUSE IN BLOCK (PERLITE EILLED) COOLING LOADS BASED ON COOLING ABOVE 78 F OUTSIDE

\begin{tabular}{|c|c|c|c|c|}
\hline $\begin{array}{l}\text { INSULATION } \\
\text { SPECS }\end{array}$ & $\begin{array}{l}\text { U } \\
\text { VALUE }\end{array}$ & $\begin{array}{l}\text { INSUL } \\
\text { COST }\end{array}$ & $\begin{array}{l}\text { ANNUAL HTG } \\
\text { (MILLION BTU) }\end{array}$ & $\begin{array}{l}\text { ANNUAL CLG } \\
\text { (MILLION BTU) }\end{array}$ \\
\hline $\begin{array}{l}\text { BASE CASE } \\
\text { CORES FILLED } \\
\text { B } 4 \\
\text { C } 4\end{array}$ & $\begin{array}{r}.249 \\
.141 \\
.104 \\
.082\end{array}$ & $\begin{array}{r}\$ \\
\$ \\
538 \\
539 \\
\$ 1109\end{array}$ & $\begin{array}{l}3.608 \\
2.025 \\
1.538 \\
1.368\end{array}$ & $\begin{array}{l}21.727 \\
20: 708 \\
20: 466 \\
20.571\end{array}$ \\
\hline $\begin{array}{l}\text { INSULATION } \\
\text { SPECS }\end{array}$ & INCREMENTAL & $\begin{array}{c}\text { INCREMENTAL } \\
\text { COST }\end{array}$ & $\begin{array}{l}\text { INCREMENTAL } \\
\text { ANNUAL HTG }\end{array}$ & $\begin{array}{l}\text { INCREMENTAL } \\
\text { ANNUAL CLG }\end{array}$ \\
\hline $\begin{array}{l}\text { CORES FILLED } \\
\text { E } 4 \\
\text { C } 4\end{array}$ & $\begin{array}{l}.108 \\
.037 \\
.022\end{array}$ & $\begin{array}{l}\$ \begin{array}{l}538 \\
101 \\
\$ 470\end{array} \\
\$ 4\end{array}$ & $\begin{array}{r}1.581 \\
.489 \\
.169\end{array}$ & $\begin{array}{r}1.019 \\
.241 \\
-.105\end{array}$ \\
\hline
\end{tabular}

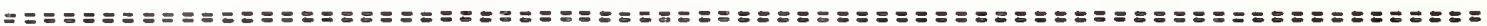
INCREMENTAL AND CUMULATIVE LIFE-CYCLE COST ANALYSES

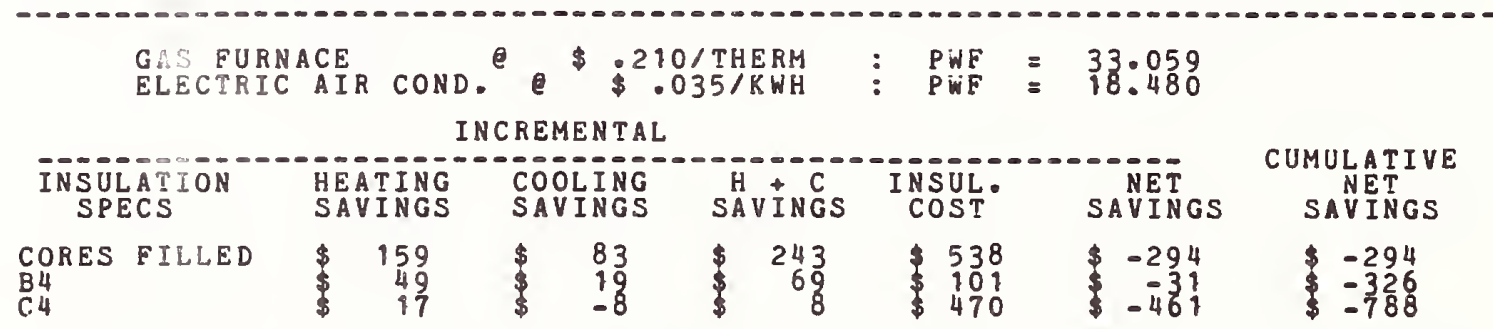

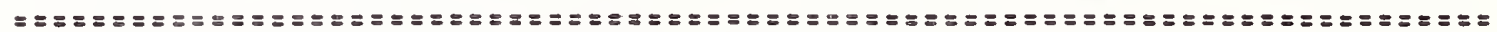

OIL FURNACE

INCREMENTAL

\begin{tabular}{|c|c|c|c|c|c|c|}
\hline SPECS & $\begin{array}{l}\text { HEAT ING } \\
\text { SAVINGS }\end{array}$ & $\begin{array}{l}\text { COOLING } \\
\text { SAVINGS }\end{array}$ & SÄVINCG & $\begin{array}{l}\text { INSUL. } \\
\text { COST }\end{array}$ & SAVINGS & SAVINGS \\
\hline $\begin{array}{l}\text { CORES FILLED } \\
\text { B } 4 \\
\text { C } 4\end{array}$ & $\begin{array}{r}183 \\
\$ \quad 16 \\
\$ \quad 19\end{array}$ & $\begin{array}{r}83 \\
19 \\
-8\end{array}$ & $\begin{array}{r}267 \\
\quad 11\end{array}$ & $\begin{array}{l}538 \\
\$ 109 \\
\$ 470\end{array}$ & $\begin{array}{l}-278 \\
\$-458\end{array}$ & $\begin{array}{l}=238 \\
=298 \\
=753\end{array}$ \\
\hline
\end{tabular}

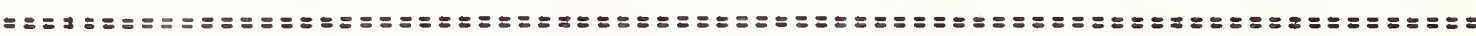

$\begin{array}{ll}\text { ELECTRIC FURNACE e } \$ .035 / K W H & \text { PWF }=18.480 \\ \text { ELECTRIC AIR COND. e } \$ .035 / K W H & : \text { PWF }=18.480\end{array}$

INCREMENTAL

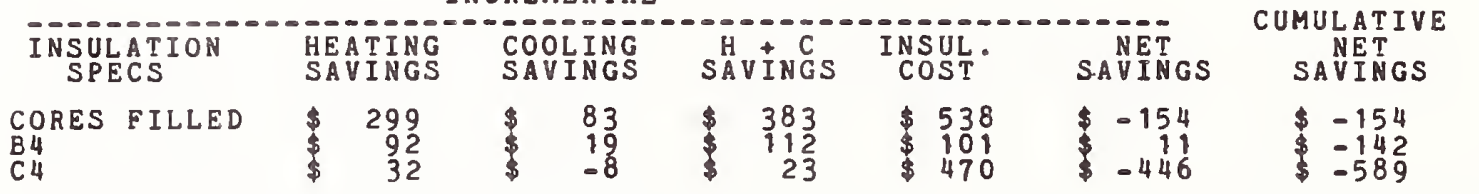

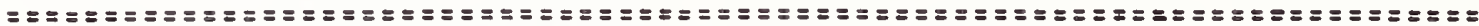

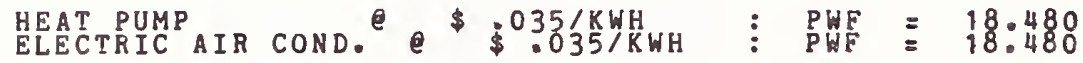

INCREMENTAL

\begin{tabular}{|c|c|c|c|c|c|c|}
\hline $\begin{array}{l}\text { INSULATION } \\
\text { SPECS }\end{array}$ & $\begin{array}{l}\text { HEATING } \\
\text { SAVINGS }\end{array}$ & $\begin{array}{l}\text { COOLING } \\
\text { SAVINGS }\end{array}$ & SAVINGS & $\begin{array}{l}\text { INSUL. } \\
\text { COST }\end{array}$ & SAVINGS & $\begin{array}{c}\text { CUMULATIVE } \\
\text { NET } \\
\text { SAVINGS }\end{array}$ \\
\hline RES FILLED & $\begin{array}{r}130 \\
40 \\
13\end{array}$ & $\begin{array}{l}87 \\
20 \\
-9\end{array}$ & 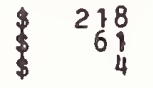 & $\begin{array}{l}538 \\
\$ 101 \\
\$ 470\end{array}$ & $\begin{array}{r}-319 \\
\$-39 \\
\$-465\end{array}$ & $\begin{array}{l}-319 \\
-359 \\
-824\end{array}$ \\
\hline
\end{tabular}

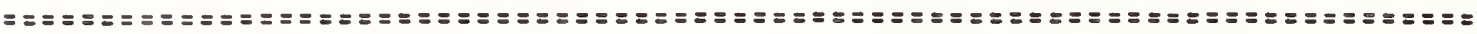


HUD 3

PHOENIX ARIZONA 8 IN BLOCK (PERLITE FILLED) COOLING LOADS BASED ON COOLING ABOVE 78 F OUTSIDE

\begin{tabular}{|c|c|c|c|c|}
\hline $\begin{array}{l}\text { INSULATION } \\
\text { SPECS }\end{array}$ & VALUE & INSUL & $\begin{array}{l}\text { ANNUAL HTC } \\
\text { MILLION }\end{array}$ & ANNUAL CLG \\
\hline $\begin{array}{l}\text { BASE CASE } \\
\text { CORES FILLED } \\
\text { B } 4 \\
\text { C4 }\end{array}$ & $\begin{array}{l}.249 \\
: 141 \\
: 104 \\
.082\end{array}$ & $\begin{array}{r}\$ 20 \\
538 \\
\$ 339 \\
\$ 1109\end{array}$ & $\begin{array}{l}4.055 \\
2.233 \\
1.680 \\
1.514\end{array}$ & $\begin{array}{l}36.678 \\
33: 102 \\
32: 005 \\
31.646\end{array}$ \\
\hline $\begin{array}{l}\text { INSULATION } \\
\text { SPECS }\end{array}$ & INCREM & $\begin{array}{c}\text { INCREMENTAL } \\
\text { COST }\end{array}$ & $\begin{array}{l}\text { INCREMENTAL } \\
\text { ANNUAL HTC }\end{array}$ & $\begin{array}{l}\text { INCREMENTAL } \\
\text { ANNUAL CLG }\end{array}$ \\
\hline $\begin{array}{l}\text { CORES FILLED } \\
\text { B } 4 \\
\text { C } 4\end{array}$ & $\begin{array}{l}.108 \\
: 037 \\
.022\end{array}$ & $\begin{array}{l}538 \\
101 \\
\$ 470\end{array}$ & $\begin{array}{r}1.822 \\
: 553 \\
: 166\end{array}$ & $\begin{array}{r}3.575 \\
1.097 \\
.359\end{array}$ \\
\hline
\end{tabular}

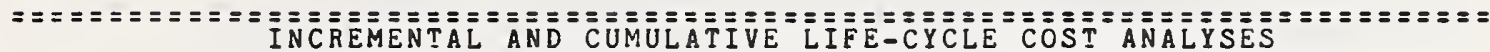

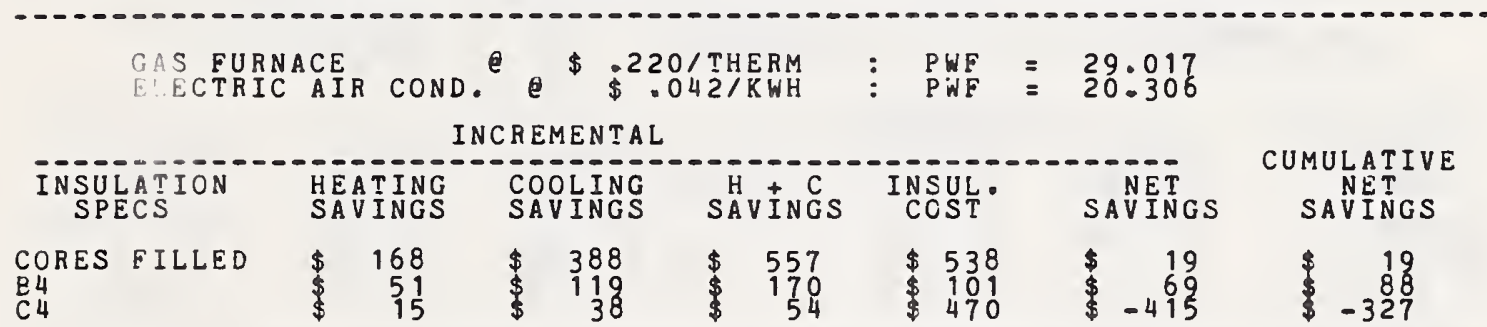

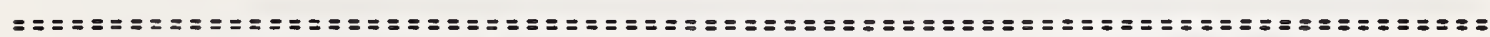

OIL FURNACE
ELECTRICAIR COND. e \$510/GALLCN $\$$ PWF $=24.167$

INCREMENTAL

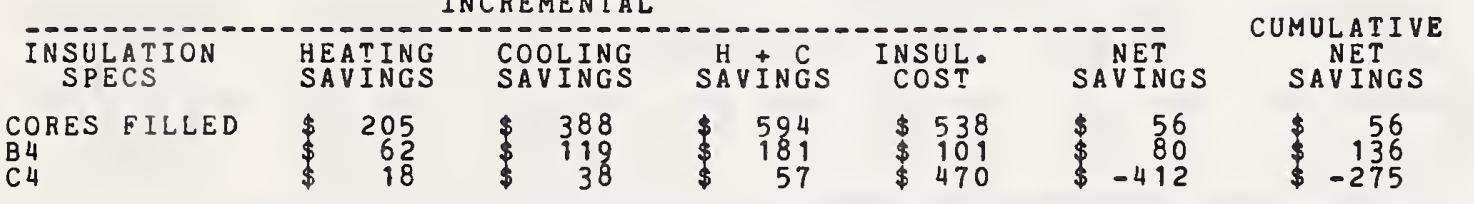

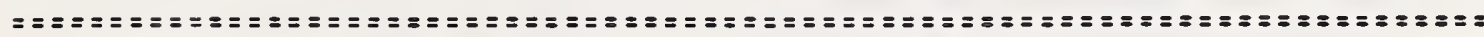

ELECTRIC FURNACE $\$$ PWF $=20.306$

INCREMENTAL

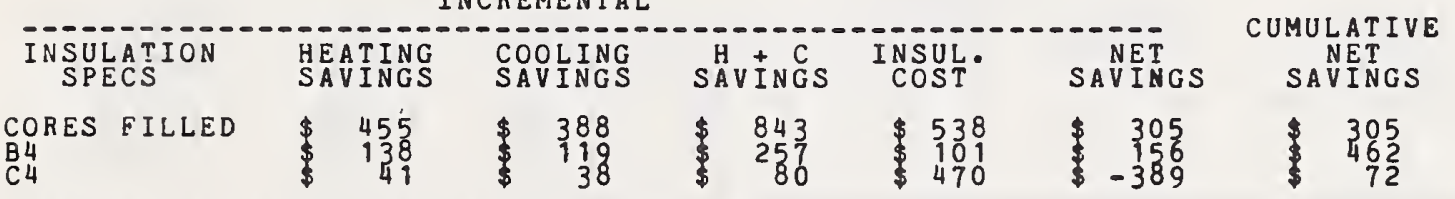

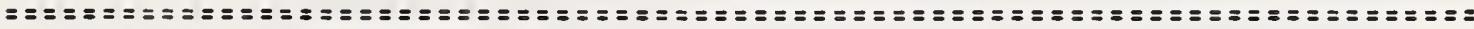

HEATPUMP
ELECTRIC AIR COND. e $\$$ \$ $\$$.042/KWH

INCREMENTAL

\begin{tabular}{|c|c|c|c|c|c|c|}
\hline $\begin{array}{l}\text { INSULATION } \\
\text { SPECS }\end{array}$ & $\begin{array}{l}\text { HEATING } \\
\text { SAVINGS }\end{array}$ & $\begin{array}{l}\text { COOLING } \\
\text { SAVINGS }\end{array}$ & SAVIINGS & INSUL. & SAVINGS & $\begin{array}{l}\text { CUMULATIVE } \\
\text { NET } \\
\text { SAVIHGS }\end{array}$ \\
\hline $\begin{array}{l}\text { CORES EILLED } \\
64 \\
64\end{array}$ & $\begin{array}{r}197 \\
60 \\
18\end{array}$ & $\begin{array}{r}406 \\
\quad 124 \\
\$ \quad 40\end{array}$ & $\begin{array}{r}604 \\
184 \\
58\end{array}$ & 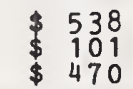 & 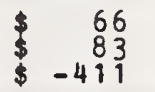 & $\begin{array}{r}\quad 66 \\
\$ 149 \\
-261\end{array}$ \\
\hline
\end{tabular}

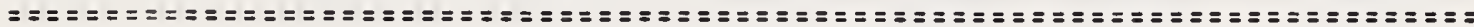


HUD 3

TAMPA FLORIDA 1200 SQ FT HOUSE 8 IN BLOCK (PERLITE FILLED)

COOLING LOADS BASED ON COOLING ABOVE 78 F OUTSIDE

\begin{tabular}{|c|c|c|c|c|}
\hline $\begin{array}{l}\text { INSULATION } \\
\text { SPECS }\end{array}$ & VALUE & $\begin{array}{l}\text { INSUL } \\
\text { COST }\end{array}$ & $\begin{array}{l}\text { ANNUAL }{ }^{\text {HTG }} \\
\text { (MILLION } \\
\text { BTU) }\end{array}$ & $\begin{array}{l}\text { ANNUAL CLG } \\
\text { (MILLION BTU) }\end{array}$ \\
\hline $\begin{array}{l}\text { PASE CASE } \\
\text { CORES FILLED } \\
\text { B } 4 \\
\text { C } 4\end{array}$ & $\begin{array}{l}.249 \\
: 147 \\
.104 \\
.082\end{array}$ & $\begin{array}{r}\$ 0 \\
\$ 338 \\
\$ 339 \\
\$ 1109\end{array}$ & $\begin{array}{r}.854 \\
.415 \\
.281 \\
.239\end{array}$ & $\begin{array}{l}24.327 \\
23: 288 \\
23: 039 \\
23.139\end{array}$ \\
\hline $\begin{array}{l}\text { INSULATION } \\
\text { SPECS }\end{array}$ & $\underset{U}{\text { INCREMENTAL }}$ & $\begin{array}{c}\text { INCREMENTAL } \\
\text { COST }\end{array}$ & $\begin{array}{l}\text { INCREMENTAL } \\
\text { ANNUAL HTG }\end{array}$ & $\begin{array}{l}\text { INCREMENTAL } \\
\text { ANNUAL CLG }\end{array}$ \\
\hline $\begin{array}{l}\text { CORES FILLED } \\
\text { B } 4 \\
\text { C } 4\end{array}$ & $\begin{array}{l}.108 \\
.037 \\
.022\end{array}$ & $\begin{array}{l}538 \\
\$ 981 \\
\$ 470\end{array}$ & $\begin{array}{r}.439 \\
.134 \\
.042\end{array}$ & $\begin{array}{r}1.039 \\
-249 \\
-.100\end{array}$ \\
\hline
\end{tabular}

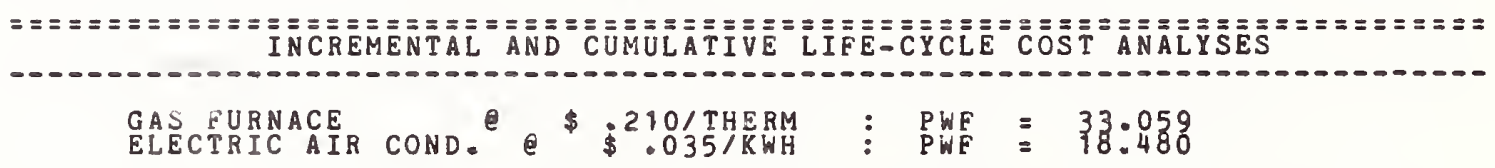

INCREMENTAL

\begin{tabular}{|c|c|c|c|c|c|c|}
\hline $\begin{array}{l}\text { INSULATION } \\
\text { SPECS }\end{array}$ & $\begin{array}{l}\text { HEATING } \\
\text { SAVINGS }\end{array}$ & $\begin{array}{l}\text { COOLING } \\
\text { SAVINGS }\end{array}$ & SAVINGS & $\begin{array}{l}\text { INSUL. } \\
\text { COST }\end{array}$ & SAVINGS & $\begin{array}{c}\text { CUMULATIVE } \\
\text { NET } \\
\text { SAVINGS }\end{array}$ \\
\hline $\int_{4}^{\text {ORES FILLED }}$ & $\begin{array}{l}44 \\
13 \\
4\end{array}$ & $\begin{array}{l}85 \\
20 \\
-8\end{array}$ & $\begin{array}{r}129 \\
33 \\
-3\end{array}$ & $\begin{array}{l}538 \\
901 \\
470\end{array}$ & $\begin{array}{r}-408 \\
-67 \\
-473\end{array}$ & $\begin{array}{l}-408 \\
\$-475 \\
-949\end{array}$ \\
\hline
\end{tabular}

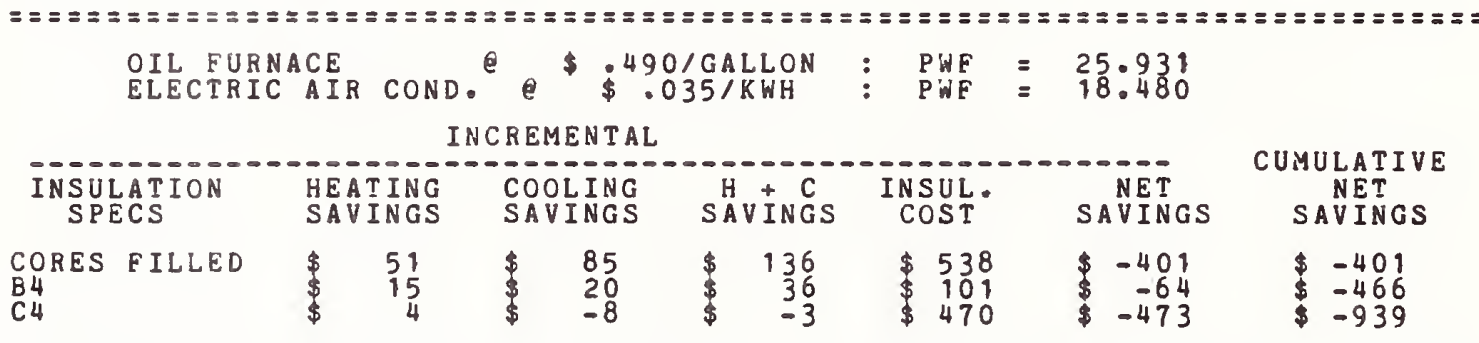

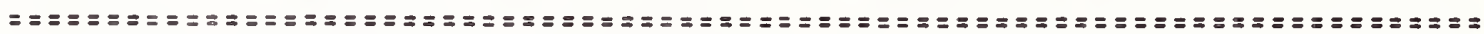

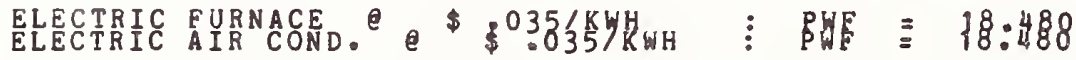
INCREMENTAL

\begin{tabular}{|c|c|c|c|c|c|c|c|c|c|}
\hline $\begin{array}{l}\text { INSULATION } \\
\text { SPECS }\end{array}$ & & $\begin{array}{l}\text { ING } \\
\text { INGS }\end{array}$ & & $\begin{array}{l}I N G \\
N G S\end{array}$ & & $\stackrel{+}{\mathrm{C}} \mathrm{C}$ & $\begin{array}{l}\text { INSUL. } \\
\text { COST }\end{array}$ & $\begin{array}{c}\text { NET } \\
\text { SAVINGS }\end{array}$ & $\begin{array}{c}\text { CUMULATIVE } \\
\text { NET } \\
\text { SAVINGS }\end{array}$ \\
\hline $\begin{array}{l}\text { CORES FILLED } \\
\text { C } 4\end{array}$ & $\$$ & $\begin{array}{r}83 \\
25 \\
7\end{array}$ & $\$$ & $\begin{array}{l}85 \\
20 \\
-8\end{array}$ & $\$$ & $\begin{array}{r}168 \\
45 \\
0\end{array}$ & $\begin{array}{l}\$ 538 \\
\$ 101 \\
\$ 470\end{array}$ & $\begin{array}{l}-369 \\
-55 \\
-470\end{array}$ & $\begin{array}{l}-369 \\
-424 \\
\$-894\end{array}$ \\
\hline
\end{tabular}

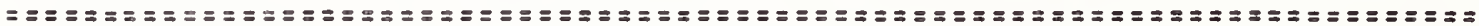

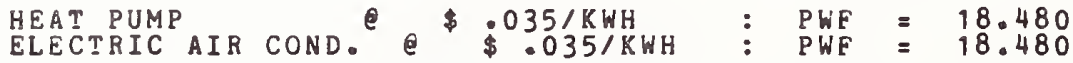
INCREMENTAL

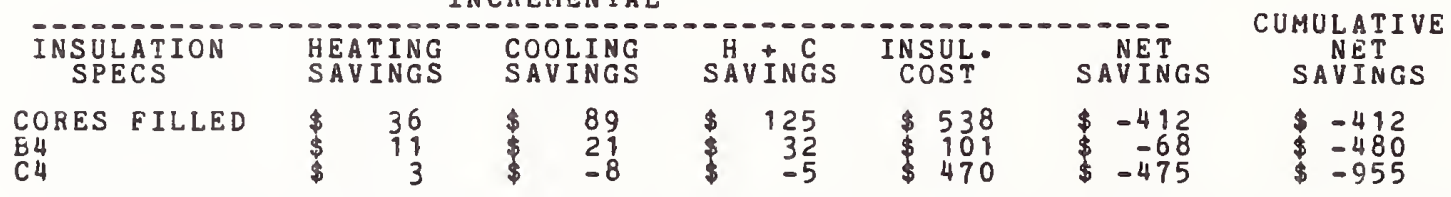


HUD 4

JACKSONVILLE FLORIDA

8 IN BLOCK (PERLITE FILLED) COOLING LOADS 1200 SQ FT HOUSE

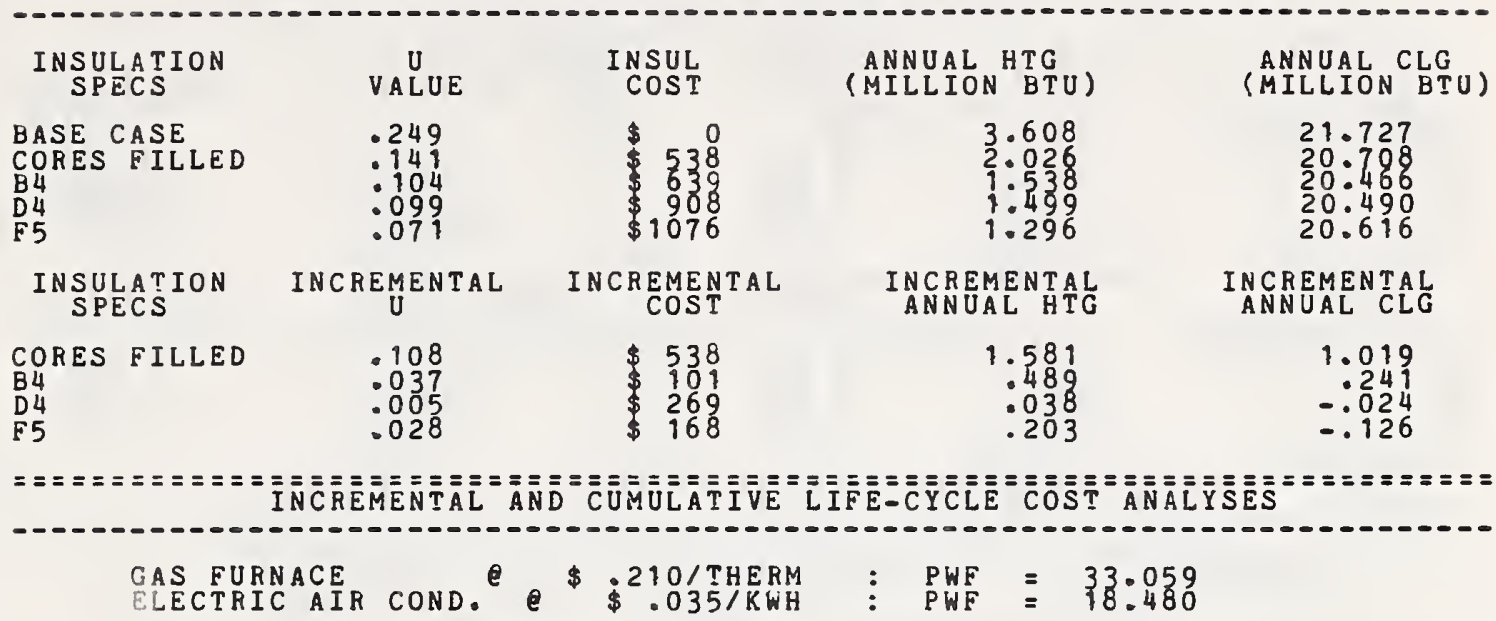

INCREMENTAL

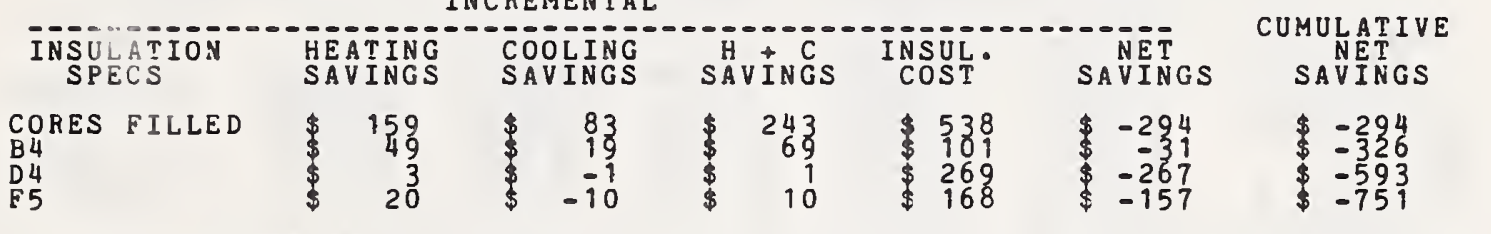

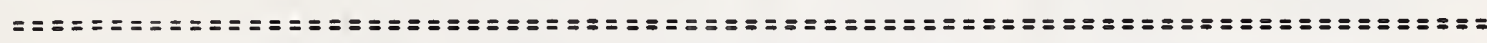

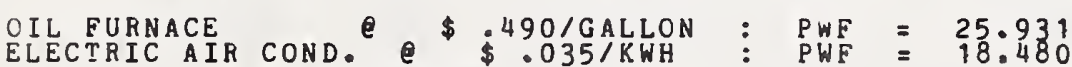

INCREMENTAL

\begin{tabular}{|c|c|c|c|c|c|c|c|c|c|}
\hline $\begin{array}{l}\text { NSULATION } \\
\text { SPECS }\end{array}$ & & $\begin{array}{l}\text { T ING } \\
\text { INGS }\end{array}$ & & $\begin{array}{l}\text { INGG } \\
\text { INGS }\end{array}$ & & VINGS & $\begin{array}{l}\text { INSUL } \\
\text { COST }\end{array}$ & SAVINGS & $\begin{array}{c}\text { CUMULATIVE } \\
\text { NET } \\
\text { SAVINGS }\end{array}$ \\
\hline ET & $\begin{array}{l}\$ \\
\$\end{array}$ & $\begin{array}{r}183 \\
56 \\
4 \\
23\end{array}$ & $\$$ & $\begin{array}{r}83 \\
19 \\
-1 \\
-10\end{array}$ & $\begin{array}{l}\$ \\
\$\end{array}$ & $\begin{array}{r}267 \\
76 \\
2 \\
13\end{array}$ & $\begin{array}{l}538 \\
101 \\
269 \\
168\end{array}$ & $\begin{array}{l}-270 \\
-24 \\
-266 \\
-154\end{array}$ & $\begin{array}{l}-270 \\
\$-294 \\
-560 \\
-715\end{array}$ \\
\hline
\end{tabular}

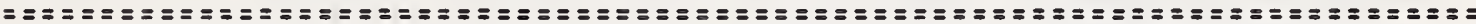

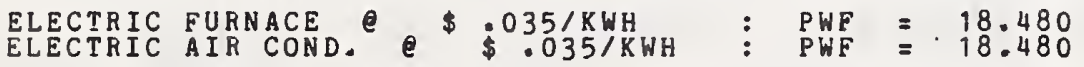
INCREMENTAL

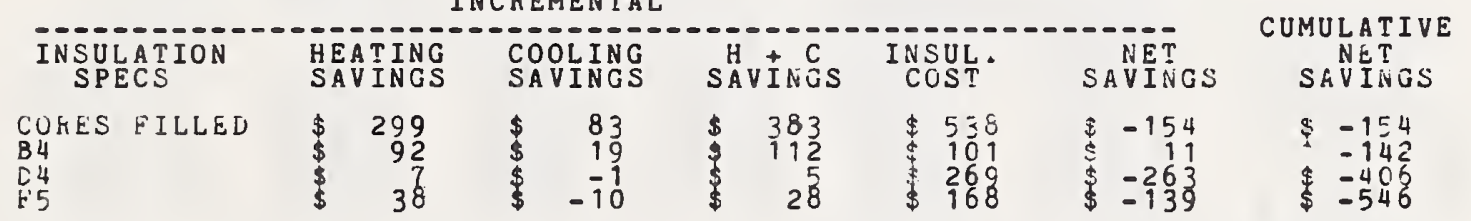

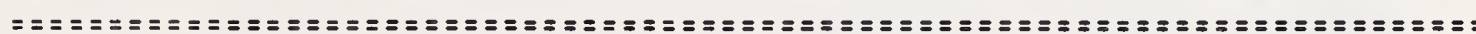

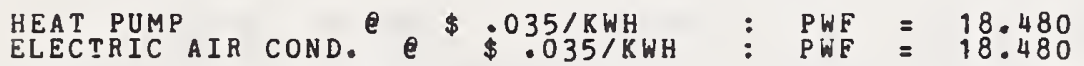

INCREMENTAL

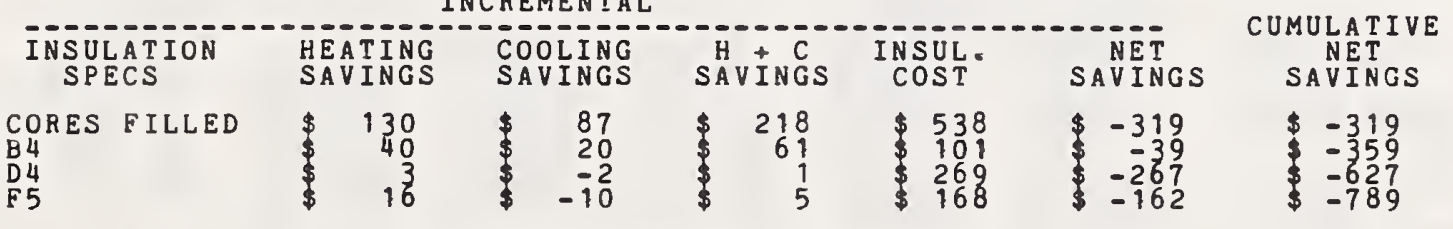

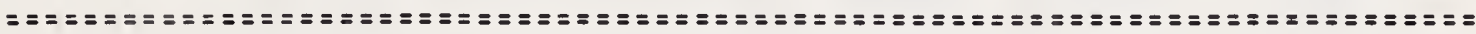


HUD 4

PHOENIX ARIZONA 1200 SQ FT HOUSE

8 IN ELOCK (PERLITE FILLED) COOLING LOADS BASED ON COOLING ABOVE 78 F OUTSIDE

\begin{tabular}{|c|c|c|c|c|}
\hline $\begin{array}{l}\text { INSULATION } \\
\text { SPECS }\end{array}$ & VALUE & $\begin{array}{l}\text { INSUL } \\
\text { COST }\end{array}$ & $\begin{array}{l}\text { ANNUAL HTG } \\
\text { (MILLION BTU) }\end{array}$ & $\begin{array}{l}\text { ANNUAL CLG } \\
\text { (MILLION BTU) }\end{array}$ \\
\hline $\begin{array}{l}\text { BASE CASE } \\
\text { CORES FILLED } \\
\text { B4 } \\
\text { D4 } \\
\text { F5 }\end{array}$ & $\begin{array}{l}.249 \\
.149 \\
.104 \\
.099 \\
.079\end{array}$ & $\begin{array}{r}\$ 0 \\
538 \\
639 \\
\$ 908 \\
\$ 1076\end{array}$ & $\begin{array}{l}4.055 \\
2.233 \\
1.680 \\
1.642 \\
1.445\end{array}$ & $\begin{array}{l}36.678 \\
33.102 \\
32.005 \\
31.924 \\
31.459\end{array}$ \\
\hline $\begin{array}{l}\text { INSULATION } \\
\text { SPECS }\end{array}$ & INCREMENTAL & $\begin{array}{c}\text { INCREMENTAL } \\
\text { COST }\end{array}$ & $\begin{array}{l}\text { INCREMENTAL } \\
\text { ANNUAL HTG }\end{array}$ & $\begin{array}{l}\text { INCKEMENTAL } \\
\text { ANNUAL CLG }\end{array}$ \\
\hline $\begin{array}{l}\text { CORES FILLED } \\
\text { B } 4 \\
\text { D4 } \\
\text { F5 }\end{array}$ & $\begin{array}{l}.108 \\
.037 \\
.005 \\
.028\end{array}$ & $\begin{array}{l}538 \\
\$ 101 \\
\$ 269 \\
\$ 168\end{array}$ & $\begin{array}{r}.822 \\
.553 \\
.038 \\
.197\end{array}$ & $\begin{array}{r}3.575 \\
1.097 \\
.082 \\
.464\end{array}$ \\
\hline
\end{tabular}

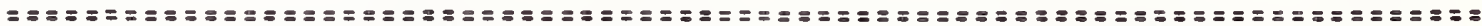
INCREMENTAL AND CUMULATIVE LIEE-CYCLE COST ANALYSES

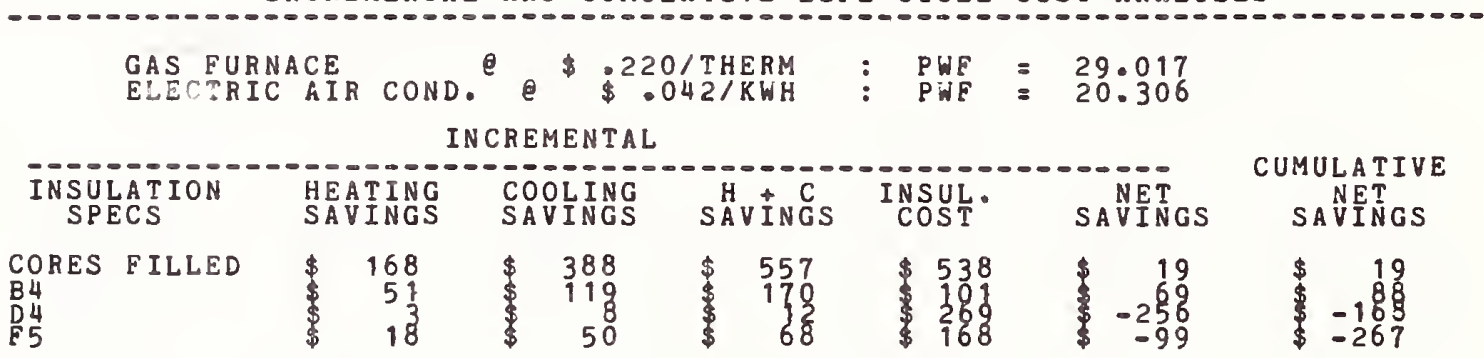

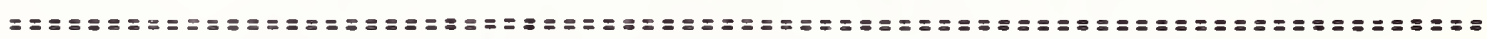

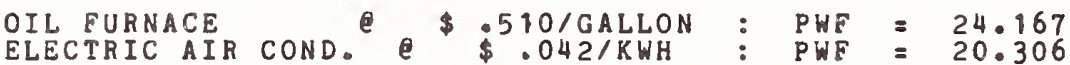

INCREMENTAL

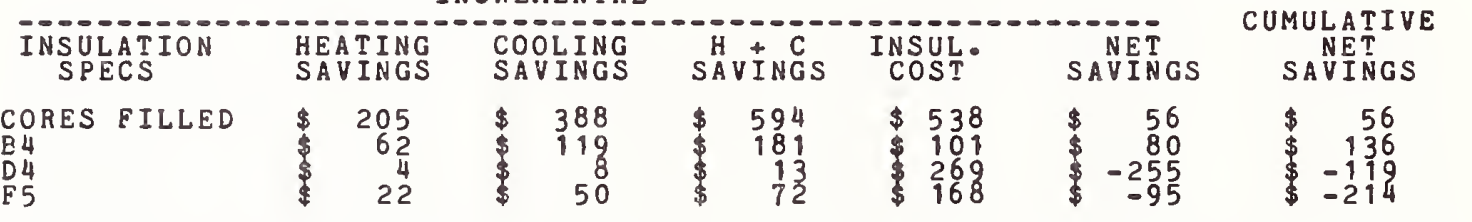

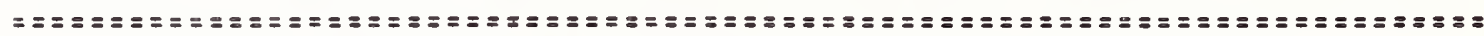

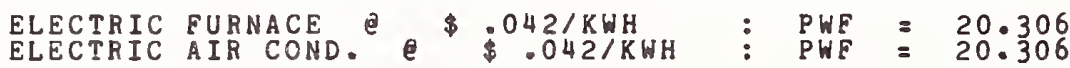

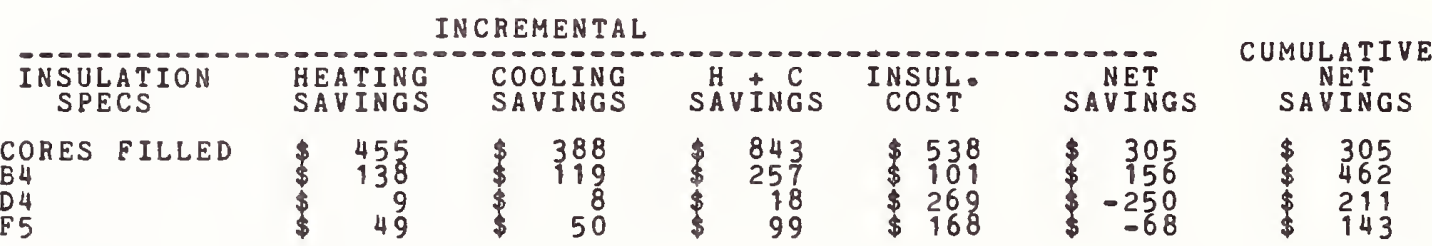

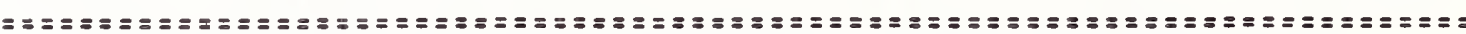
HEAT PUMP
ELECTRIC AIR COND. e \$ $\$ 042 / K W H W H$

INCREMENTAL

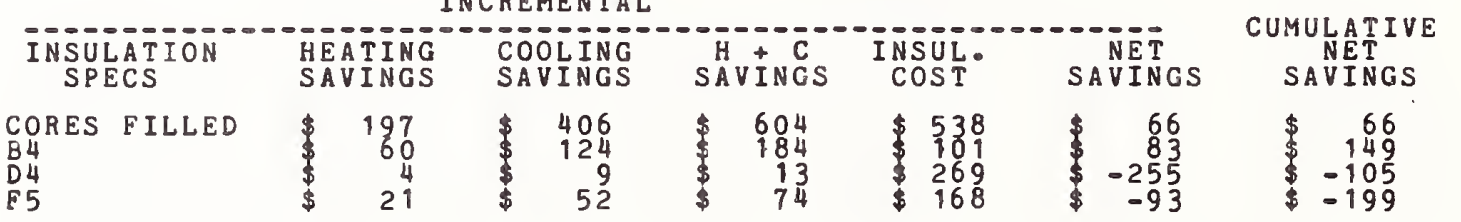


HUD 4

TAMPA FLORIDA $\$ 200$ SQ FT HOUSE 8 IN BLOCK (PERLITE FILLED) COOLING LOADS BASED ON COOLING ABOVE 78 F OUTSIDE

\begin{tabular}{|c|c|c|c|c|}
\hline $\begin{array}{l}\text { INSULATION } \\
\text { SPECS }\end{array}$ & $\stackrel{U}{\text { VALUE }}$ & $\begin{array}{l}\text { INSUL } \\
\text { COST }\end{array}$ & $\begin{array}{l}\text { ANNUAL HTG } \\
\text { (MILLION BTU) }\end{array}$ & $\begin{array}{l}\text { ANNUAL CLG } \\
\text { (MILLION BTU) }\end{array}$ \\
\hline $\begin{array}{l}\text { BASE CASE } \\
\text { CORES FILLED } \\
\text { B } 4 \\
\text { D } 4 \\
\text { F5 }\end{array}$ & $\begin{array}{l}.249 \\
.149 \\
.104 \\
.099 \\
.071\end{array}$ & $\begin{array}{r}53 \\
538 \\
539 \\
908 \\
\$ 1076\end{array}$ & $\begin{array}{r}.854 \\
.495 \\
.281 \\
.271 \\
.223\end{array}$ & $\begin{array}{l}24 \cdot 327 \\
23: 288 \\
23: 039 \\
23.062 \\
23 \cdot 182\end{array}$ \\
\hline $\begin{array}{l}\text { INSULATION } \\
\text { SPECS }\end{array}$ & INCREMENTAL & $\begin{array}{c}\text { INCREMENTAL } \\
\text { COST }\end{array}$ & $\begin{array}{l}\text { INCREMENTAL } \\
\text { ANNUAL HTG }\end{array}$ & $\begin{array}{l}\text { INCHEMENTAL } \\
\text { ANNUAL CLF; }\end{array}$ \\
\hline $\begin{array}{l}\text { CORES FILLED } \\
\text { B } 4 \\
\text { D } 4 \\
\text { F5 }\end{array}$ & $\begin{array}{l}.108 \\
.037 \\
.005 \\
.028\end{array}$ & $\begin{array}{l}538 \\
\$ 109 \\
\$ 269 \\
\$ 168\end{array}$ & $\begin{array}{r}.439 \\
.134 \\
.010 \\
.048\end{array}$ & $\begin{array}{r}1.039 \\
.249 \\
-.023 \\
-.120\end{array}$ \\
\hline
\end{tabular}

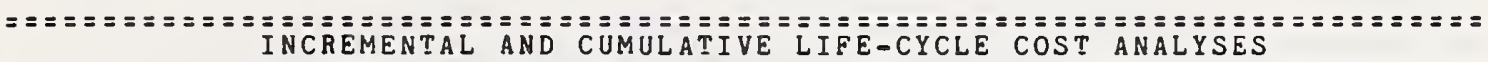

GAS FURNACE
ELECTRIC AIR COND.

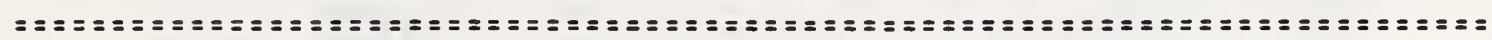
ELECTRIC FURNACE e $e^{\$ 035 / K W H}$ \$ $\$ 3$ PWF $=18.480$
ELECTRIC AIR COND.

INCREMENTAL

\begin{tabular}{|c|c|c|c|c|c|c|}
\hline $\begin{array}{l}\text { SPECS } \\
\text { SPEN }\end{array}$ & $\begin{array}{l}\text { HEATING } \\
\text { SAVINGS }\end{array}$ & $\begin{array}{l}\text { COOLING } \\
\text { SAVINGS }\end{array}$ & $\stackrel{H}{\text { SAV I NGS }}$ & $\begin{array}{l}\text { INSUL. } \\
\text { COST }\end{array}$ & $\begin{array}{l}\text { NET } \\
\text { SAVINGS }\end{array}$ & $\begin{array}{l}\text { CUHULATIVE } \\
\text { NET } \\
\text { SAVINGS }\end{array}$ \\
\hline $\begin{array}{l}\text { CORES FILLED } \\
\text { B } 4 \\
\text { D4 } \\
\text { F5 }\end{array}$ & $\begin{array}{r}83 \\
25 \\
1 \\
9\end{array}$ & $\begin{array}{l}85 \\
20 \\
-1 \\
-9\end{array}$ & $\begin{array}{r}168 \\
45 \\
0 \\
0\end{array}$ & $\begin{array}{l}538 \\
\$ 101 \\
\$ 269 \\
168\end{array}$ & $\begin{array}{l}-369 \\
-55 \\
-269 \\
-168\end{array}$ & $\begin{array}{l}\$-369 \\
-424 \\
\$-693 \\
-862\end{array}$ \\
\hline
\end{tabular}

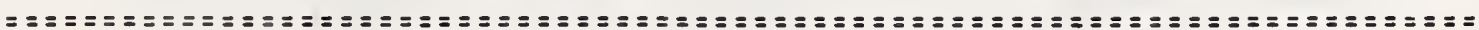

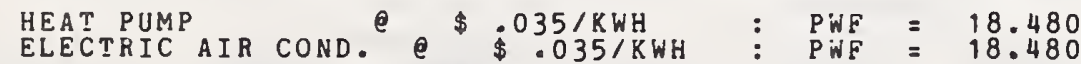
INCREMENTAL

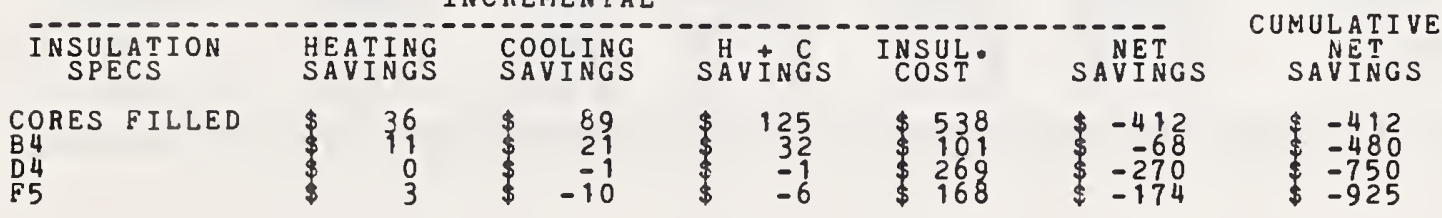

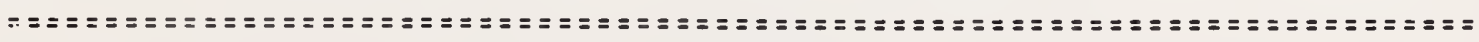


HUDS

JACKSONVILLE FLORIDA, 200 SO FT HOUSE FOD FRE WALL COOLING LOADS BASED ON COOLING ABOVE 78 F OUTSIDE

\begin{tabular}{|c|c|c|c|c|}
\hline $\begin{array}{l}\text { INSULATION } \\
\text { SPECS }\end{array}$ & VALUE & $\begin{array}{l}\text { INSUL } \\
\text { COST }\end{array}$ & 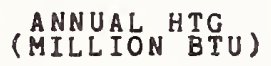 & $\begin{array}{l}\text { ANNUAL CLG } \\
\text { (MILLION BTU) }\end{array}$ \\
\hline $\begin{array}{l}\text { BASE CASE } \\
R=11 \\
R=13 \\
R=19\end{array}$ & $\begin{array}{l}.218 \\
.082 \\
.076 \\
.055\end{array}$ & $\begin{array}{lr}\$ & 0 \\
\$ & 213 \\
\$ & 280 \\
& 504\end{array}$ & $\begin{array}{l}3.721 \\
1: 752 \\
1: 668 \\
1.393\end{array}$ & $\begin{array}{l}23.181 \\
21: 524 \\
21: 450 \\
21: 184\end{array}$ \\
\hline $\begin{array}{l}\text { INSULATION } \\
\text { SPECS }\end{array}$ & INCREM $\underset{U}{ }$ ENTAL & $\begin{array}{c}\text { INCREMENTAL } \\
\text { COST }\end{array}$ & $\begin{array}{l}\text { INCREMENTAL } \\
\text { ANNUAL HTG }\end{array}$ & $\begin{array}{l}\text { INCREMENTAL } \\
\text { ANNUAL CLG }\end{array}$ \\
\hline $\begin{array}{l}R-11 \\
R=13 \\
R=19\end{array}$ & $\begin{array}{l}.136 \\
.006 \\
.021\end{array}$ & $\begin{array}{r}213 \\
\$ \quad 67 \\
\$ \quad 224\end{array}$ & $\begin{array}{r}1.969 \\
.084 \\
.275\end{array}$ & $\begin{array}{r}1.656 \\
.074 \\
.266\end{array}$ \\
\hline
\end{tabular}

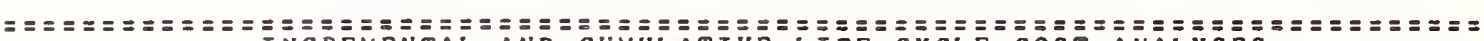
INCREMENTAL AND CUMULATIVE LIEE-CYCLE COST ANALYSES

GAS FURNACE
ELECTRICAIR COND. e e $\$ 210 /$ THERM
$\$$ PWF

INCREMENTAL

\begin{tabular}{|c|c|c|c|c|c|c|}
\hline $\begin{array}{l}\text { INSULATION } \\
\text { SPECS }\end{array}$ & $\begin{array}{l}\text { HEATING } \\
\text { SAVINGS }\end{array}$ & $\begin{array}{l}\text { COOLING } \\
\text { SAVINGS }\end{array}$ & SAVINGS & INSUL. & SAVET INGS & $\begin{array}{c}\text { CUMULATIVE } \\
\text { NETT } \\
\text { SAVNGS }\end{array}$ \\
\hline $\begin{aligned}-11 \\
-13\end{aligned}$ & $\begin{array}{r}198 \\
\$ \quad 8 \\
\$ \quad 27\end{array}$ & $\begin{array}{r}136 \\
6 \\
21\end{array}$ & $\begin{array}{r}334 \\
14 \\
49\end{array}$ & $\begin{array}{r}213 \\
\$ 227\end{array}$ & $\begin{array}{r}121 \\
\$-172 \\
\$-174\end{array}$ & $\begin{array}{r}121 \\
\$ \quad 69 \\
\$-105\end{array}$ \\
\hline
\end{tabular}

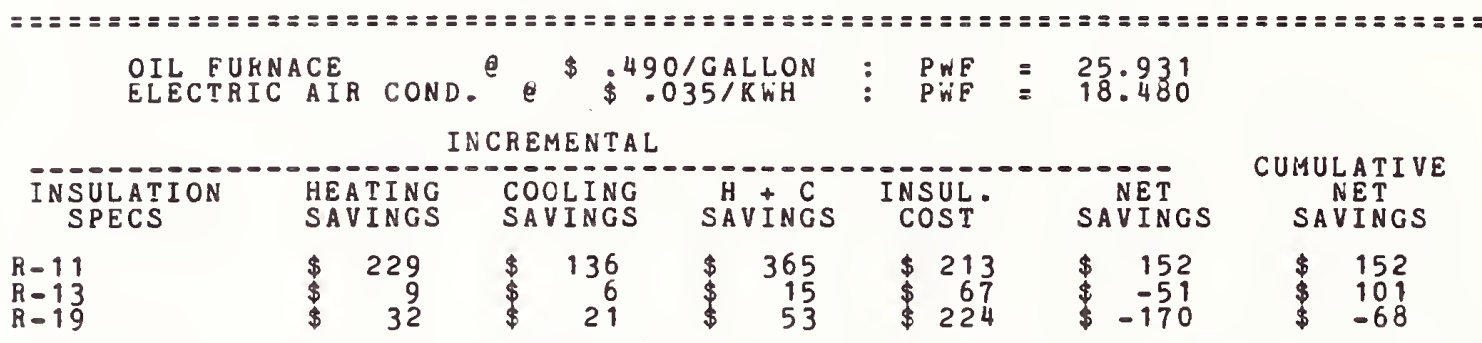

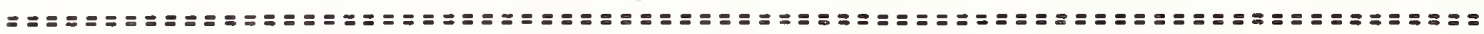

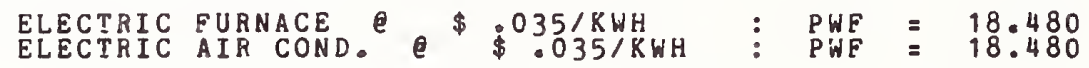

I NCREMENTAL

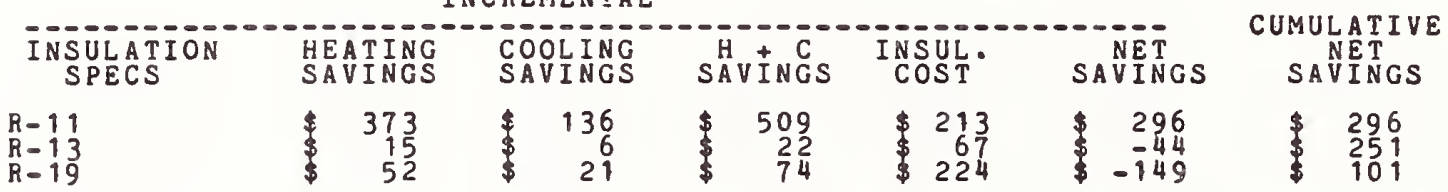

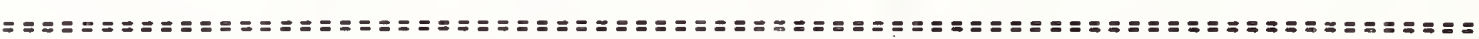

HEAT PUMP
ELECTRIC AIR COND. e $\$ .035 / K W H$

INCREMENTAL

\begin{tabular}{|c|c|c|c|c|c|c|}
\hline $\begin{array}{l}\text { INSULATION } \\
\text { SPECS }\end{array}$ & $\begin{array}{l}\text { HEAT INC } \\
\text { SAVINGS }\end{array}$ & $\begin{array}{l}\text { COOLING } \\
\text { SAVINGS }\end{array}$ & $\stackrel{H}{\text { SAVINGS }}$ & $\begin{array}{l}\text { INSUL. } \\
\text { COST }\end{array}$ & SAVINGS & $\begin{array}{c}\text { CUMULATIVE } \\
\text { LETT } \\
\text { SAVINGS }\end{array}$ \\
\hline $\begin{array}{r}-11 \\
-13 \\
-19\end{array}$ & $\begin{array}{r}162 \\
6 \\
22\end{array}$ & $\begin{array}{r}142 \\
6 \\
22\end{array}$ & $\begin{array}{r}304 \\
13 \\
45\end{array}$ & $\begin{array}{r}213 \\
\$ 27 \\
\$ 224\end{array}$ & $\begin{array}{r}91 \\
-53 \\
-178\end{array}$ & $\begin{array}{r}91 \\
\$ 38 \\
-140\end{array}$ \\
\hline
\end{tabular}


HUD5

PHOENIX ARIZONA WOOD FRAME WALL COOLING LOADS BASED ON COOLING ABOVE 78 F OUTSIDE

\begin{tabular}{|c|c|c|c|c|}
\hline $\begin{array}{l}\text { INSULATION } \\
\text { SPECS }\end{array}$ & $\stackrel{U}{\text { VALUE }}$ & $\begin{array}{l}\text { INSUL } \\
\text { COST }\end{array}$ & $\begin{array}{l}\text { ANNUAL HTG } \\
\text { (MILLION BTU) }\end{array}$ & $\begin{array}{l}\text { ANNUAL CLG } \\
\text { (MILLION BTU) }\end{array}$ \\
\hline $\begin{array}{l}\text { BASE CASE } \\
R=11 \\
R=13 \\
R=19\end{array}$ & $\begin{array}{l}.218 \\
.082 \\
.076 \\
.055\end{array}$ & $\begin{array}{rr} & 0 \\
& 213 \\
& 280 \\
& 280 \\
5 & 504\end{array}$ & $\begin{array}{l}4.479 \\
2.079 \\
1.975 \\
1.625\end{array}$ & $\begin{array}{l}37.733 \\
32.939 \\
32: 718 \\
31.883\end{array}$ \\
\hline $\begin{array}{l}\text { INSULATION } \\
\text { SPECS }\end{array}$ & INCREMUENTAL & INCREMEMTTAL & $\begin{array}{l}\text { INCRE:ENTAL } \\
\text { ANNUAL HTG }\end{array}$ & $\begin{array}{l}\text { INCREMENTAL } \\
\text { ANNUAL CLG }\end{array}$ \\
\hline $\begin{array}{l}R=11 \\
R=13 \\
R=19\end{array}$ & $\begin{array}{r}136 \\
.006 \\
.021\end{array}$ & $\begin{array}{r}213 \\
\$ 27 \\
\$ 224\end{array}$ & $\begin{array}{r}2.400 \\
.104 \\
.350\end{array}$ & $\begin{array}{r}4.794 \\
: 221 \\
.836\end{array}$ \\
\hline
\end{tabular}

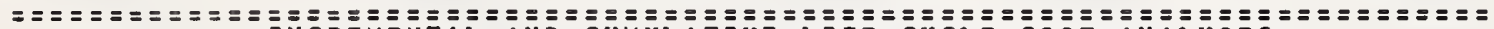
INCAEMENTAL AND CUMULATIVE LIFE-CYCLE COST ANALYSES

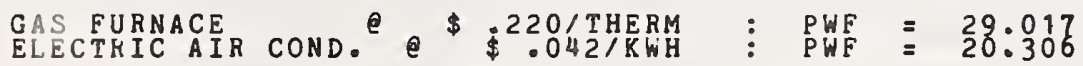

INCREMENTAL

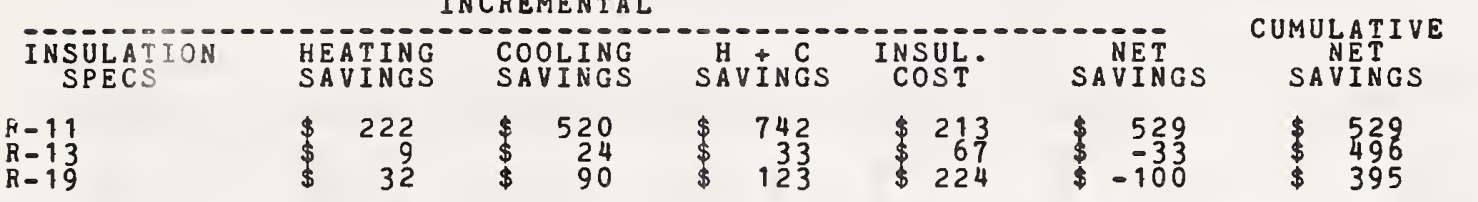

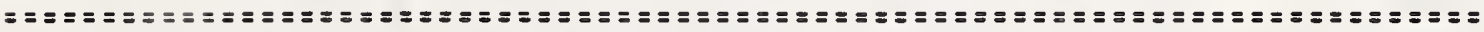

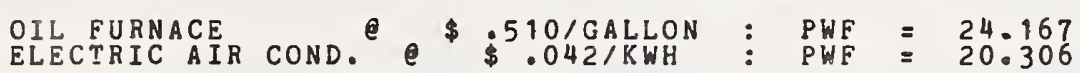

INCREMENTAL

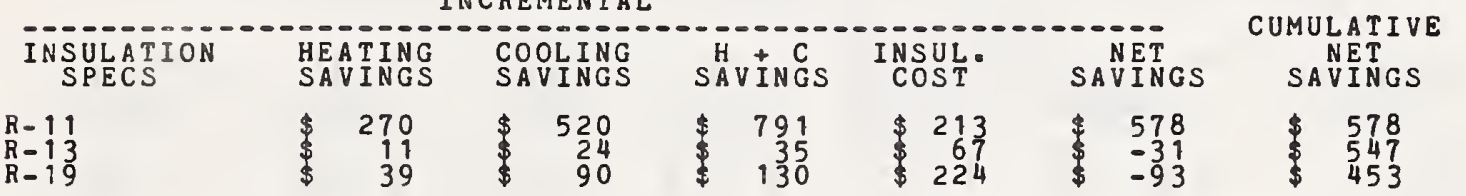

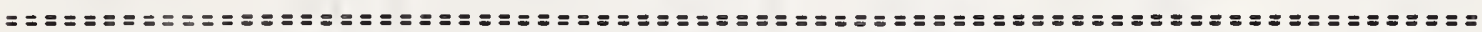

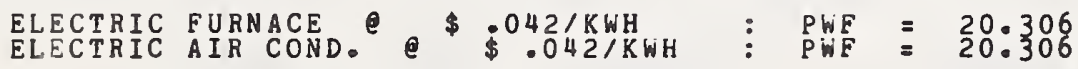

INCREMENTAL

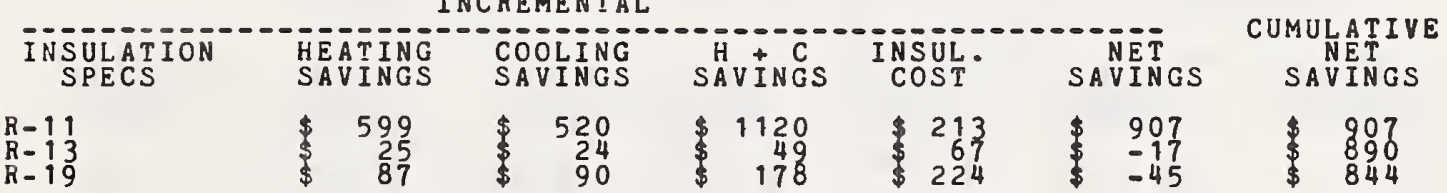

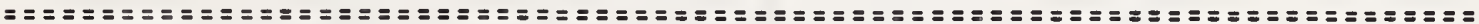
HEAT PUMP
ELECTRIC AIR COND. e $\$ 042 / K W H$ INCREMENTAL

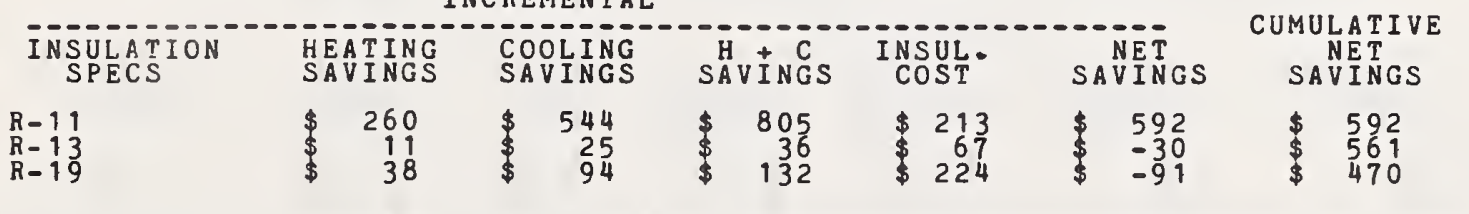


HUD 5

TAMPA FLORIDA 1200 SQ FT HOUSE WOOD FRAME WALL

COOLING LOADS BASED ON COOLING ABOVE 78 F OUTSIDE

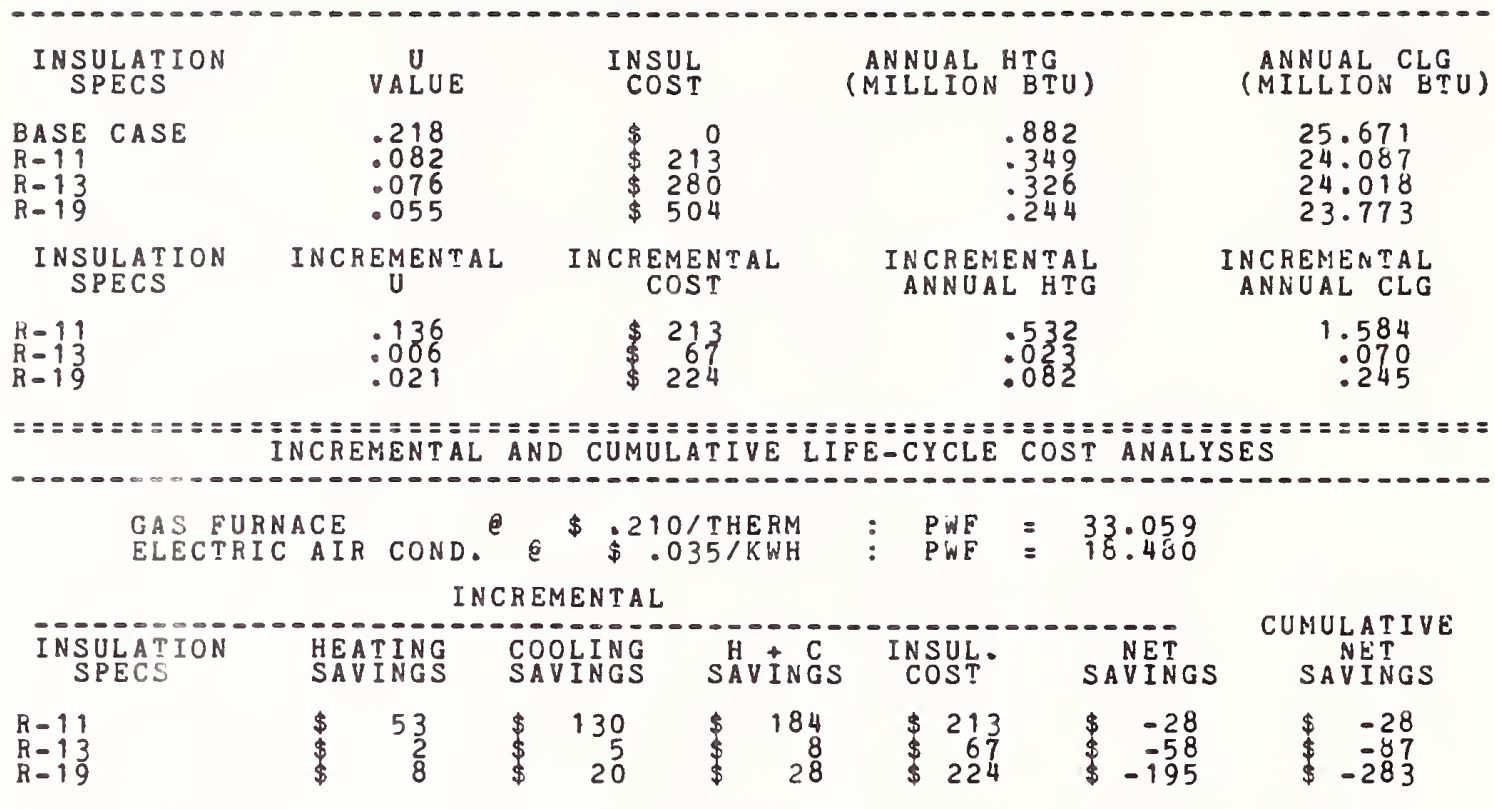

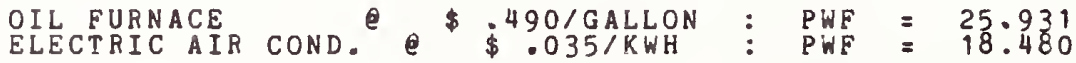

INCREMENTAL

\begin{tabular}{|c|c|c|c|c|c|c|}
\hline $\begin{array}{l}\text { INSULATION } \\
\text { SPECS }\end{array}$ & $\begin{array}{l}\text { HEATING } \\
\text { SAVINGS }\end{array}$ & $\begin{array}{l}\text { COOLING } \\
\text { SAVINGS }\end{array}$ & SAVV IN $\stackrel{+}{C}$ & $\begin{array}{l}\text { INSUL. } \\
\text { COST }\end{array}$ & SAVVINGS & $\begin{array}{l}\text { CUHOLATIVE } \\
\text { SAVIET }\end{array}$ \\
\hline 11 & $\begin{array}{r}61 \\
2 \\
9\end{array}$ & $\begin{array}{r}130 \\
5 \\
20\end{array}$ & $\begin{array}{r}192 \\
8 \\
29\end{array}$ & $\begin{array}{r}213 \\
\$ \quad 67 \\
\$ 224\end{array}$ & $\begin{array}{r}-20 \\
\$-58 \\
-194\end{array}$ & $\begin{array}{r}-20 \\
\$-279\end{array}$ \\
\hline
\end{tabular}

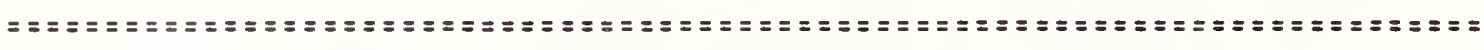

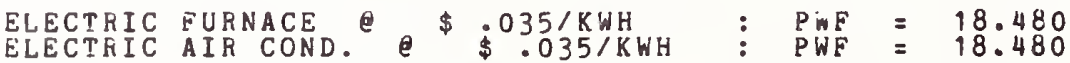

I NCREMENTAL

\begin{tabular}{|c|c|c|c|c|c|c|}
\hline $\begin{array}{l}\text { INSULATION } \\
\text { SPECS }\end{array}$ & $\begin{array}{l}\text { HEATING } \\
\text { SAVINGS }\end{array}$ & $\begin{array}{l}\text { COOLING } \\
\text { SAVINGS }\end{array}$ & $\stackrel{H}{\text { SAV I INGS }}$ & $\begin{array}{l}\text { INSUL. } \\
\text { COST }\end{array}$ & $\begin{array}{l}\text { NET } \\
\text { SAVINGS }\end{array}$ & $\begin{array}{l}\text { UNULATIVE } \\
\text { NET } \\
\text { SAVINGS }\end{array}$ \\
\hline .11 & $\begin{array}{r}100 \\
4 \\
15\end{array}$ & $\begin{array}{r}130 \\
5 \\
20\end{array}$ & $\begin{array}{rr} & 231 \\
& 10 \\
& \\
& 35\end{array}$ & $\begin{array}{r}213 \\
\$ 27 \\
\$ 224\end{array}$ & $\begin{array}{rr}\$ & 18 \\
\$ & -56 \\
\$ & -188\end{array}$ & $\begin{array}{r}18 \\
\$-226\end{array}$ \\
\hline
\end{tabular}

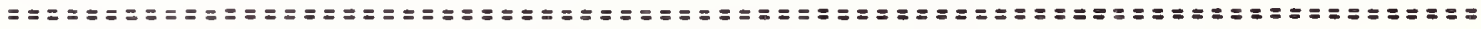

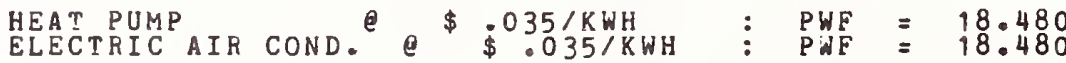

INCREMENTAL

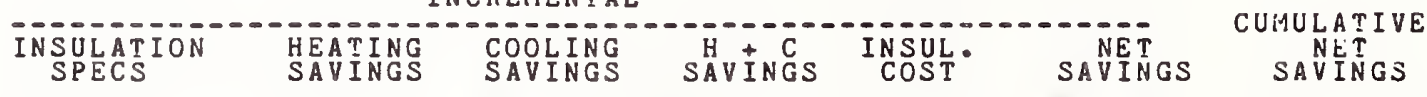

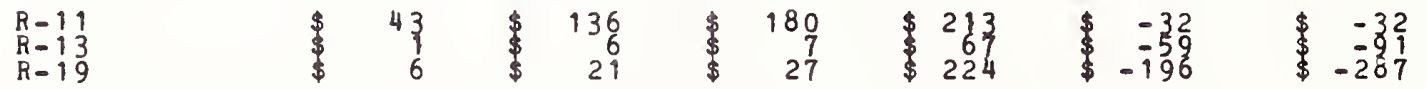




\section{APPENDIX D \\ Computer Printouts of Economic Analyses \\ for Madison, Phoenix and Washington, D.C. \\ Assuming $90^{\circ}$ Rotation of Building}


HUD 1

MADISON GSCONSIN COOLING LOADS BASED ON NO COOLING SAVINGS

90 DEGREE ROTATION (A CASE)

\begin{tabular}{|c|c|c|c|c|}
\hline $\begin{array}{l}\text { INSULATION } \\
\text { SPECS }\end{array}$ & VALUE & $\begin{array}{l}\text { INSUL } \\
\text { COST }\end{array}$ & $\begin{array}{l}\text { ANNUAL }{ }^{H T G} \\
\text { (NILLION BTU) }\end{array}$ & $\begin{array}{l}\text { ANNUAL CLG } \\
\text { (HILLIUN BTU) }\end{array}$ \\
\hline $\begin{array}{l}\text { BASE CASE } \\
\text { B } \\
\text { C } 3 \\
\text { EXTRA }\end{array}$ & $\begin{array}{l}.249 \\
.153 \\
.110 \\
.095\end{array}$ & $\begin{array}{rr}\$ & 0 \\
\$ & 101 \\
\$ & 571 \\
\$ & 597\end{array}$ & $\begin{array}{l}51.917 \\
39: 702 \\
34: 126 \\
32.172\end{array}$ & $\begin{array}{l}6.513 \\
6: 513 \\
6: 513 \\
6.513\end{array}$ \\
\hline $\begin{array}{l}\text { INSULAT ION } \\
\text { SPECS }\end{array}$ & INCREMENTAL & $\begin{array}{c}\text { INCREMENTAL } \\
\text { COST }\end{array}$ & $\begin{array}{l}\text { INCREMENTAL } \\
\text { ANNUAL HTG }\end{array}$ & $\begin{array}{l}\text { INCREMENTAL } \\
\text { ANNUAL CLG }\end{array}$ \\
\hline $\begin{array}{l}\text { B } 3 \\
\text { C } 3 \\
\text { EXTRA }\end{array}$ & $\begin{array}{l}.096 \\
.043 \\
.015\end{array}$ & $\begin{array}{rr}\$ & 101 \\
\$ & 470 \\
\$ & 26\end{array}$ & $\begin{array}{r}12.215 \\
5.576 \\
1.954\end{array}$ & $\begin{array}{l}.000 \\
.000 \\
.000\end{array}$ \\
\hline
\end{tabular}

= = INCREMENTAL AND CUMULATIVE LIFE-CYCLE COST ANALYSES

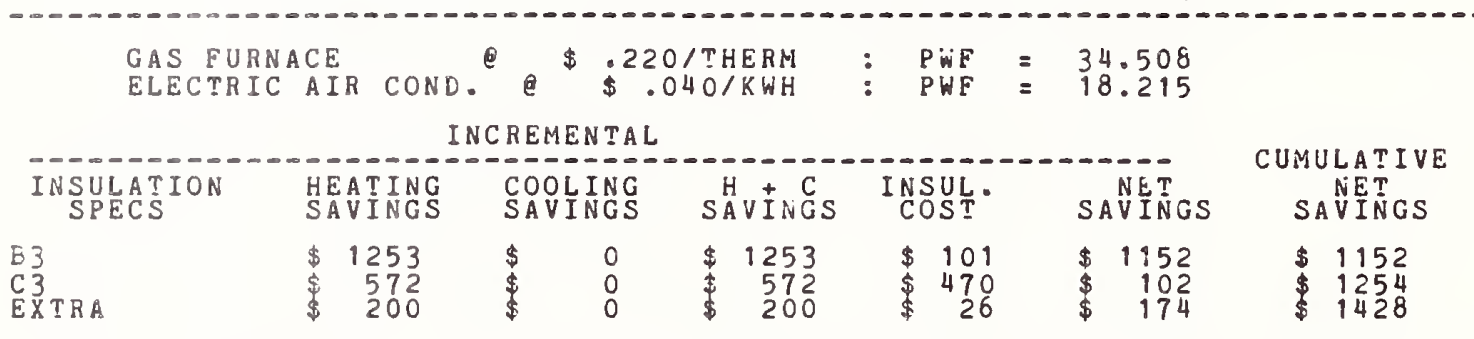

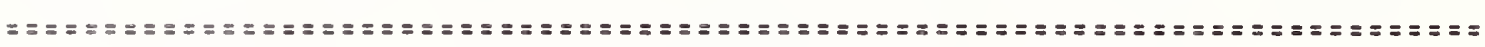

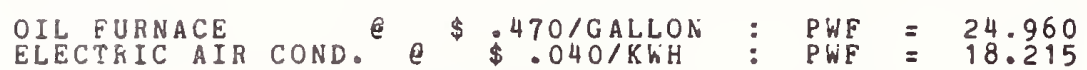
INCREIENTAL

\begin{tabular}{|c|c|c|c|c|c|c|}
\hline $\begin{array}{l}\text { INSULAT ION } \\
\text { SPECS }\end{array}$ & $\begin{array}{l}\text { HEATING } \\
\text { SAVINGS }\end{array}$ & $\begin{array}{l}\text { COOLING } \\
\text { SAVINGS }\end{array}$ & SAY $^{H}+\underset{I}{C}$ & $\begin{array}{l}\text { INSUL. } \\
\text { CCST }\end{array}$ & SAVINGS & $\begin{array}{l}\text { CUMULATIVE } \\
\text { NETINGS }\end{array}$ \\
\hline$T$ & $\begin{array}{r}1346 \\
614 \\
215\end{array}$ & $\begin{array}{l}0 \\
0 \\
0\end{array}$ & $\begin{array}{r}1346 \\
514 \\
\$ 295\end{array}$ & $\begin{array}{r}101 \\
\$ 470 \\
\$ 26\end{array}$ & $\begin{array}{r}1245 \\
\$ 144 \\
189\end{array}$ & $\begin{array}{l}1245 \\
\$ 1389 \\
1579\end{array}$ \\
\hline
\end{tabular}

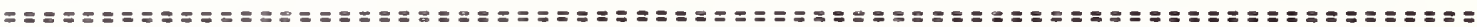

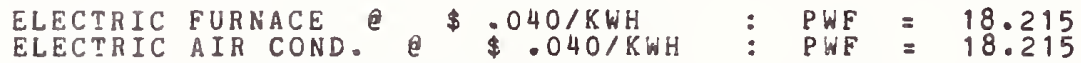
INCREMENTAL

\begin{tabular}{|c|c|c|c|c|c|c|}
\hline $\begin{array}{l}\text { INSULATION } \\
\text { SPECS }\end{array}$ & $\begin{array}{l}\text { HEATING } \\
\text { SAVINGS }\end{array}$ & $\begin{array}{l}\text { COOLING } \\
\text { SAVINGS }\end{array}$ & SÄV $\stackrel{+}{I} \stackrel{C}{N G S}$ & $\begin{array}{l}\text { INSUL. } \\
\text { COST }\end{array}$ & SAVINGS & SAVINGS \\
\hline TRA & $\begin{array}{r}2607 \\
\$ 1190 \\
\$ \quad 417\end{array}$ & $\$$ & $\begin{array}{r}2607 \\
1190 \\
\$ \quad 417\end{array}$ & $\begin{array}{r}101 \\
\$ 470 \\
\$ \quad 26\end{array}$ & $\begin{array}{r}2506 \\
\quad 720 \\
\$ 391\end{array}$ & $\begin{array}{l}\$ 2506 \\
\$ 3226 \\
\$ 3618\end{array}$ \\
\hline
\end{tabular}

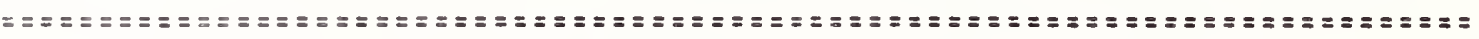

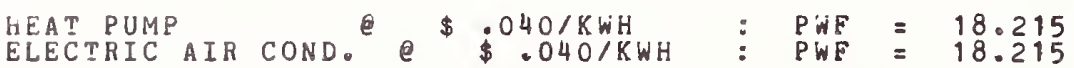
INCREMENTAL

\begin{tabular}{|c|c|c|c|c|c|c|}
\hline $\begin{array}{l}\text { INSULATION } \\
\text { SPECS }\end{array}$ & $\begin{array}{l}\text { HEATING } \\
\text { SAVINGS }\end{array}$ & $\begin{array}{l}\text { COOLING } \\
\text { SAVINGS }\end{array}$ & SÄVIN ${ }^{+}$CGS & INSUL? & SAVINGS & $\begin{array}{l}\text { CURULATE } \\
\text { SAVINGS }\end{array}$ \\
\hline$T R A$ & $\begin{array}{r}1682 \\
767 \\
\$ 269\end{array}$ & $\$$ & $\begin{array}{r}1682 \\
767 \\
269\end{array}$ & $\begin{array}{r}101 \\
\$ 470 \\
\$ \quad 26\end{array}$ & $\begin{array}{r}1581 \\
\$ 297 \\
\$ \quad 243\end{array}$ & $\begin{array}{l}1581 \\
\$ 1879 \\
\$ 2122\end{array}$ \\
\hline
\end{tabular}


HUD I

PHOENIX ARIZON/200 SO FT HOUSE 90 DEGREE ROTATION (A CASE) COOLING LOADS BASED ON NO COOLING SAVINGS

\begin{tabular}{|c|c|c|c|c|}
\hline $\begin{array}{l}\text { INSULATION } \\
\text { SPECS }\end{array}$ & VALUE & $\begin{array}{l}\text { INSUL } \\
\text { COST }\end{array}$ & $\begin{array}{l}\text { ANNUAL HTG } \\
\text { (MILLION BTU) }\end{array}$ & $\begin{array}{l}\text { ANNUAL CLG } \\
\text { (MILLION BTU) }\end{array}$ \\
\hline $\begin{array}{l}\text { BASE CASE } \\
\text { B } 3 \\
\text { E } 3 \text { TRA }\end{array}$ & $\begin{array}{r}.249 \\
: 153 \\
.190 \\
.095\end{array}$ & $\begin{array}{r}\$ 0 \\
\$ 101 \\
\$ 571 \\
\$ 597\end{array}$ & $\begin{array}{l}4.762 \\
3.245 \\
2.622 \\
2.410\end{array}$ & $\begin{array}{l}44.675 \\
44.675 \\
44.675 \\
44.675\end{array}$ \\
\hline $\begin{array}{l}\text { INSULATION } \\
\text { SPECS }\end{array}$ & INCREMENTAL & $\begin{array}{c}\text { INCREMENTAL } \\
\text { COST }\end{array}$ & $\begin{array}{l}\text { INCREMENTAL } \\
\text { ANNUAL HTG }\end{array}$ & $\begin{array}{l}\text { INCKEMENTAL } \\
\text { ANNUAL CLG }\end{array}$ \\
\hline $\begin{array}{l}\text { B3 } \\
C 3 \\
\text { EXTRA }\end{array}$ & $\begin{array}{l}.096 \\
.043 \\
.015\end{array}$ & $\begin{array}{r}109 \\
\$ 470 \\
\$ \quad 26\end{array}$ & $\begin{array}{r}1.518 \\
.623 \\
.212\end{array}$ & $\begin{array}{l}.000 \\
.000 \\
.000\end{array}$ \\
\hline
\end{tabular}

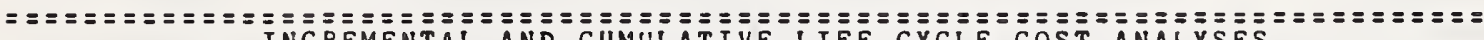
INCREMENTAL AND CUMULATIVE LIFE-CYCLE COST ANALYSES

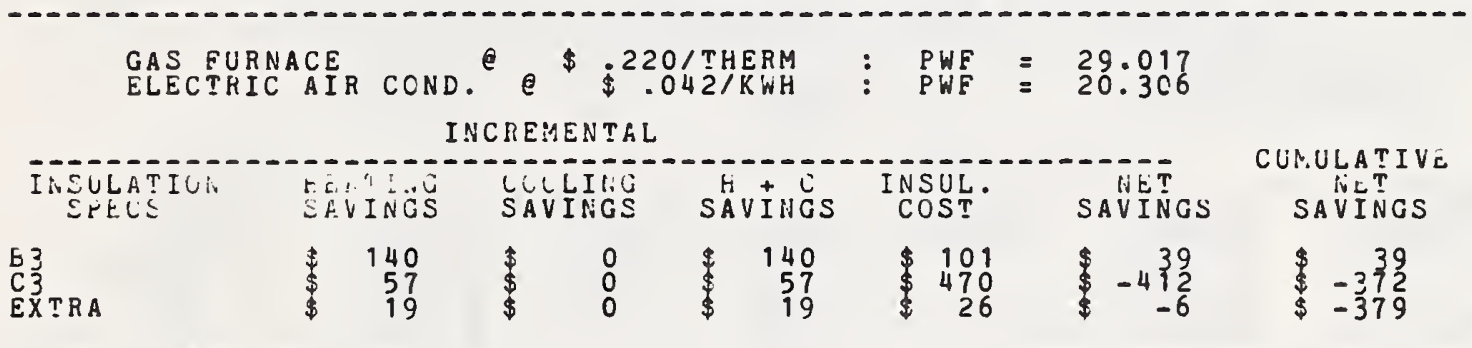

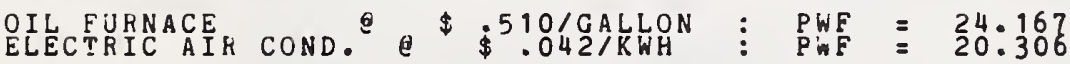

INCREMENTAL

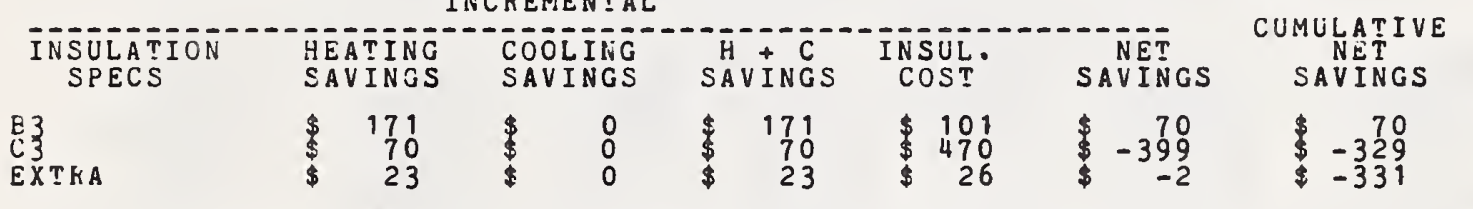

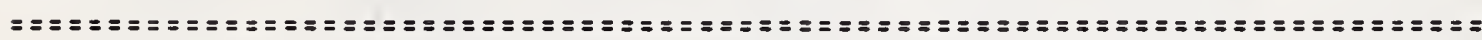

ELECTRIC FURNACE
ELECTKIC AIR COND. $\$ .042 / K W H$
$\$$ PWF

INCREMENTAL

\begin{tabular}{|c|c|c|c|c|c|c|c|}
\hline $\begin{array}{l}\text { INSULATION } \\
\text { SPECS }\end{array}$ & $\begin{array}{l}\text { HEATING } \\
\text { SAVINGS }\end{array}$ & $\begin{array}{l}\text { COOLING } \\
\text { SAVINGS }\end{array}$ & SAVINCS & $\begin{array}{l}\text { INSUL. } \\
\text { COST }\end{array}$ & SAVINGS & $\begin{array}{r}\text { CUMU } \\
\text { SA }\end{array}$ & $\begin{array}{l}\text { LATIVE } \\
\text { NET } \\
\text { VINGS }\end{array}$ \\
\hline THA & $\begin{array}{r}379 \\
\$ \quad 155 \\
\quad 53\end{array}$ & $\$$ & $\begin{array}{r}379 \\
\$ \\
\$ 55 \\
53\end{array}$ & $\begin{array}{r}101 \\
\$ 470 \\
\$ 26\end{array}$ & $\begin{array}{r}278 \\
-314 \\
\$ \quad 27\end{array}$ & $\$$ & $\begin{array}{r}278 \\
-36 \\
-9\end{array}$ \\
\hline
\end{tabular}

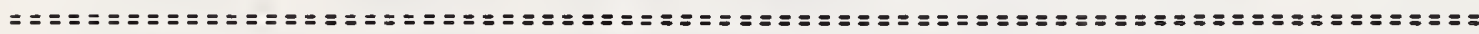
HEAT PUMP

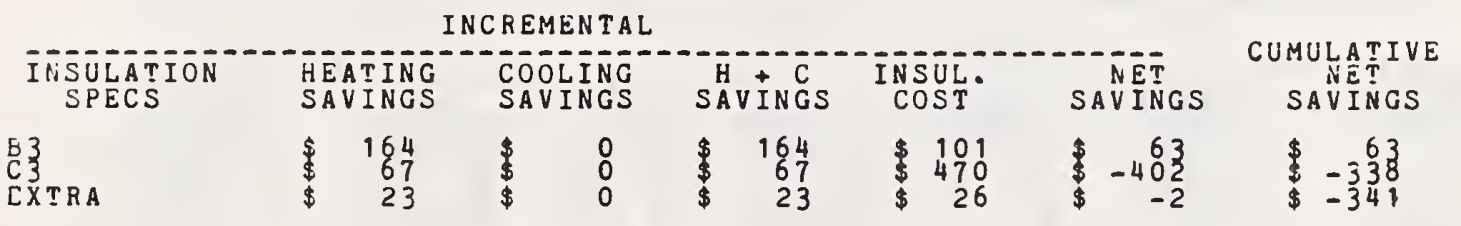

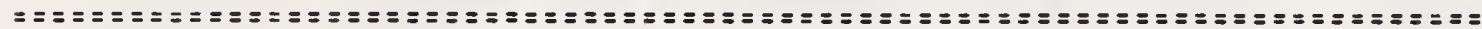


HUD 1

WASHINGTON D.C.

90 DEGREE ROTATION (A CASE) COOLING LOADS BASED ON NOHOUSE TOLING SAVINGS

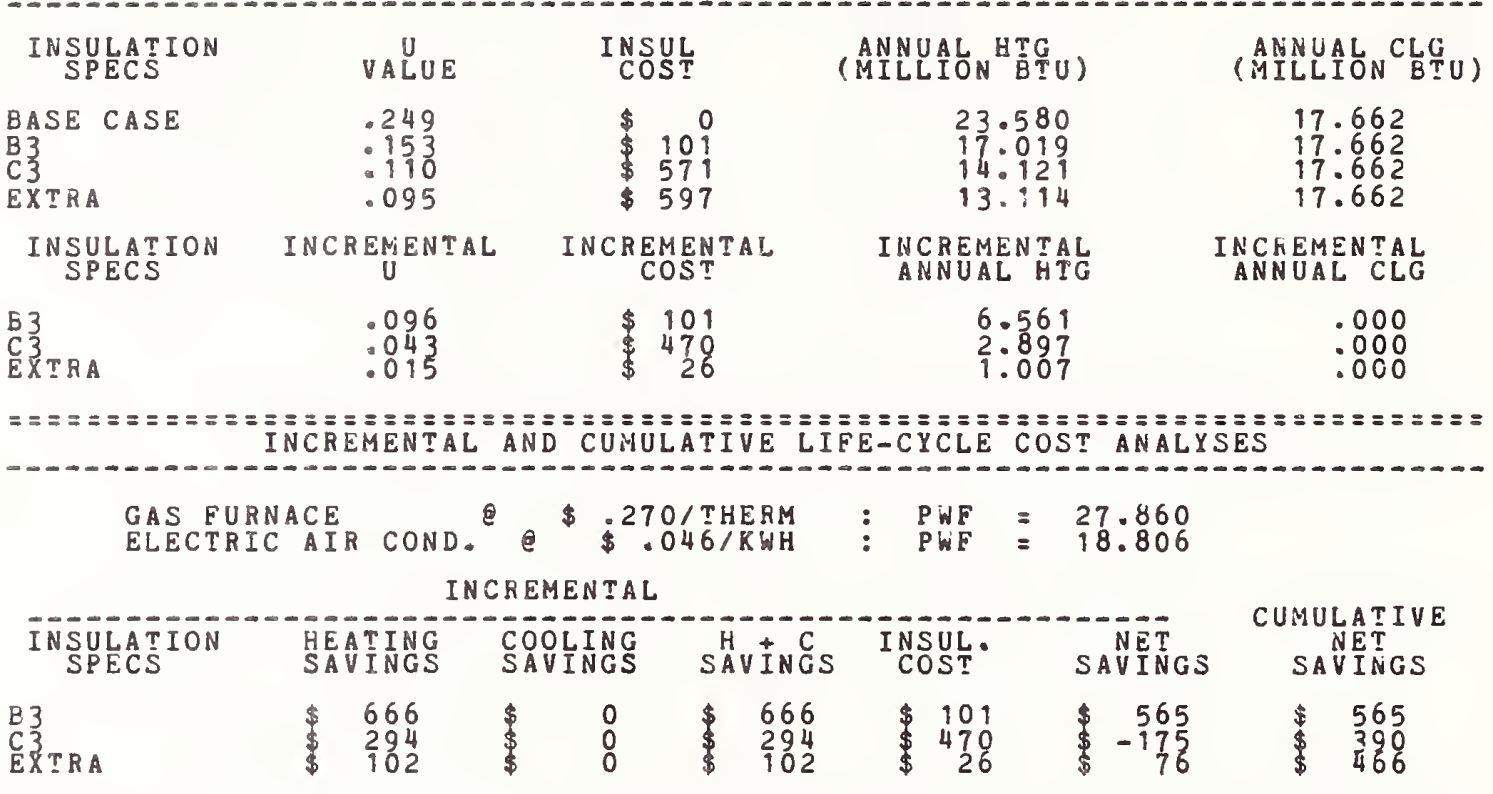

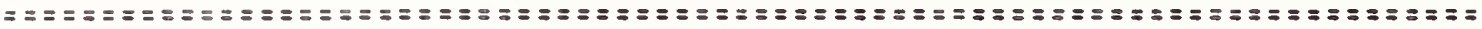

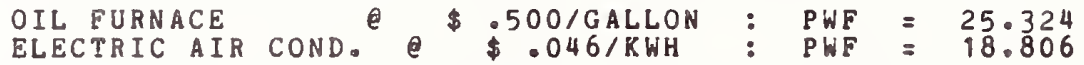

INCREMENTAL

\begin{tabular}{|c|c|c|c|c|c|c|}
\hline $\begin{array}{l}\text { IN SULATION } \\
\text { SPECS }\end{array}$ & $\begin{array}{l}\text { HEATING } \\
\text { SAVINGS }\end{array}$ & $\begin{array}{l}\text { COOLING } \\
\text { SAVINGS }\end{array}$ & SÂV İNGS & INSUL. & SAVINGS & $\begin{array}{c}\text { CUMULATIVE } \\
\text { NET } \\
\text { SAVINGS }\end{array}$ \\
\hline $\begin{array}{l}3 \\
3 \\
X T R A\end{array}$ & $\begin{array}{l}\quad \begin{array}{l}760 \\
\$ 35\end{array} \\
\$ \quad 316\end{array}$ & $\$$ & $\begin{array}{l}\$ 760 \\
\$ \quad 335 \\
\$ \quad 36\end{array}$ & $\begin{array}{r}101 \\
\$ 470 \\
\$ 26\end{array}$ & $\begin{array}{r}\quad 659 \\
\$-134 \\
\$ \quad 90\end{array}$ & $\begin{array}{l}\$ \quad 659 \\
\$ \quad 525 \\
\$ \quad 616\end{array}$ \\
\hline
\end{tabular}

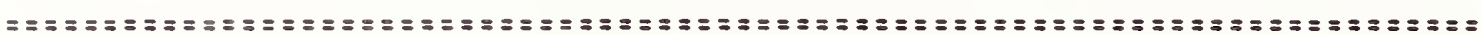

ELECTRIC FURNACE e $\$ \$ 046 / K W H$
ELECTRIC AIR COND. e $\$ .046 / K W H$

INCREMENTAL

\begin{tabular}{|c|c|c|c|c|c|c|}
\hline $\begin{array}{l}\text { INSULATION } \\
\text { SPECS }\end{array}$ & $\begin{array}{l}\text { HEATING } \\
\text { SAVINGS }\end{array}$ & $\begin{array}{l}\text { COOLING } \\
\text { SAVINGS }\end{array}$ & SAUVINGS & INSUL. & SAV INGS & $\begin{array}{l}\text { JMULATIVE } \\
\text { NET } \\
\text { SAVINGS }\end{array}$ \\
\hline TRA & $\begin{array}{r}1663 \\
\quad 734 \\
255\end{array}$ & $\begin{array}{l}0 \\
0 \\
0\end{array}$ & $\begin{array}{r}1663 \\
734 \\
255\end{array}$ & $\begin{array}{r}101 \\
\$ 479 \\
\$ 26\end{array}$ & $\begin{array}{r}1562 \\
264 \\
229\end{array}$ & $\begin{array}{l}1562 \\
\$ 1826 \\
\$ 2055\end{array}$ \\
\hline
\end{tabular}

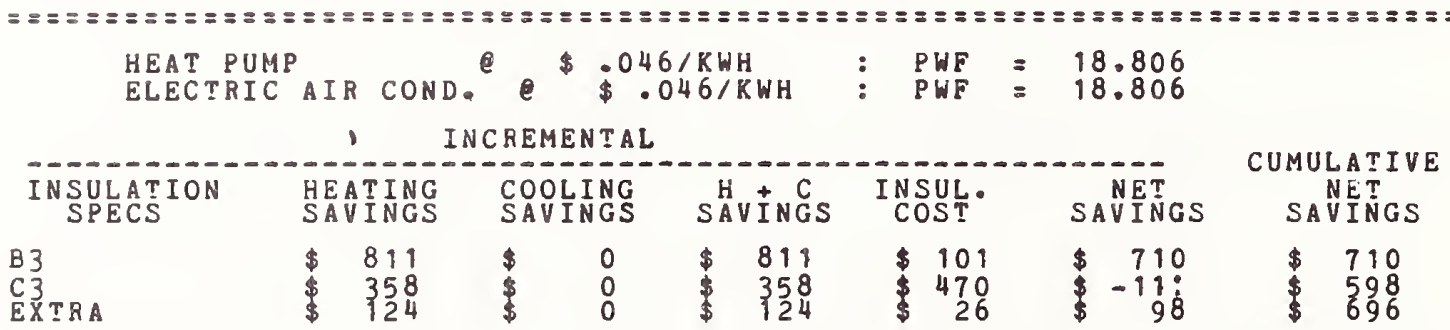

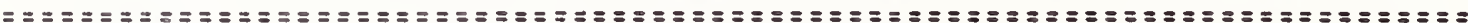


HUD 2

MADISON WISCONSIN 90 DEGREE ROTATION (A CASE) COOLING LOADS BASED ON NO HOUSE COOLING SAVINGS

\begin{tabular}{|c|c|c|c|c|}
\hline $\begin{array}{l}\text { INSULATION } \\
\text { SPECS }\end{array}$ & VALUE & $\begin{array}{l}\text { INSUL } \\
\text { COST }\end{array}$ & $\begin{array}{l}\text { ANNUAL } \\
(M T L C T O N \\
\text { MILU) }\end{array}$ & ( ANNUAL CLATU) \\
\hline $\begin{array}{l}\text { BASE CASE } \\
\text { B } \\
\text { D } 3 \\
\text { E2 }\end{array}$ & $\begin{array}{r}.249 \\
.153 \\
.143 \\
.091\end{array}$ & $\begin{array}{r}\$ 0 \\
\$ 101 \\
3770 \\
\$ 538\end{array}$ & $\begin{array}{l}51.917 \\
39: 702 \\
38.426 \\
31.651\end{array}$ & $\begin{array}{l}6.513 \\
6.513 \\
6: 513 \\
6.513\end{array}$ \\
\hline $\begin{array}{l}\text { INSULATION } \\
\text { SPECS }\end{array}$ & INCKEM & INCREMENTAL' & $\begin{array}{l}\text { INCREMENTAL } \\
\text { ANNUAL }\end{array}$ & $\begin{array}{l}\text { INCKEMENTAL } \\
\text { ANNUAL CLG }\end{array}$ \\
\hline $\begin{array}{l}\text { E3 } \\
D 3 \\
\text { E2 }\end{array}$ & $\begin{array}{l}.096 \\
.010 \\
.052\end{array}$ & $\begin{array}{l}\$ 101 \\
\$ 269 \\
\$ 168\end{array}$ & $\begin{array}{r}12.215 \\
1: 276 \\
6: 775\end{array}$ & $\begin{array}{l}.000 \\
.000 \\
.000\end{array}$ \\
\hline
\end{tabular}

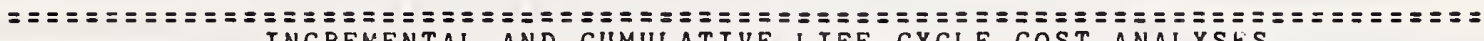
INCREMENTAL AND CUMULATIVE LIFE-CYCLE COST ANALYSES

GAS FURNACE
GAS FURNACE
ELECTRICAIR COND. $\$ \$ 220 /$ THERM $\$$ PWF $=34.508$

INCREMENTAL

\begin{tabular}{|c|c|c|c|c|c|c|}
\hline $\begin{array}{l}\text { NSULAT T I N } \\
\text { SPECS }\end{array}$ & $\begin{array}{l}\text { HEATINC } \\
\text { SAVINGS }\end{array}$ & $\begin{array}{l}\text { COOLINC } \\
\text { SAVINGS }\end{array}$ & $\begin{array}{l}\text { HU士CCS } \\
\text { SAVINGS }\end{array}$ & INSUL. & SAVINGS & $\begin{array}{c}\text { CUNULATIVE } \\
\text { NET } \\
\text { SAVINGS }\end{array}$ \\
\hline & $\begin{array}{r}\$ 1253 \\
\$ \quad 130 \\
\$ \quad 695\end{array}$ & $\$$ & $\begin{array}{r}\$ 1253 \\
\$ \quad 130 \\
\$ \quad 695\end{array}$ & $\begin{array}{l}\$ 101 \\
\$ 269 \\
\$ 168\end{array}$ & $\begin{array}{r}1152 \\
-138 \\
527\end{array}$ & 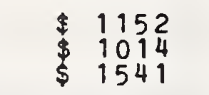 \\
\hline
\end{tabular}

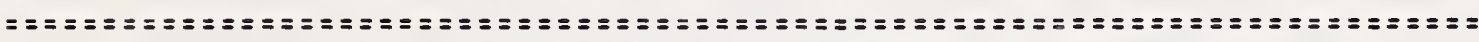

OIL EURNACE $\$$ PWF $=24.960$

INCFEMENTAL

\begin{tabular}{|c|c|c|c|c|c|c|}
\hline $\begin{array}{l}\text { INSULAT ION } \\
\text { SPECS }\end{array}$ & $\begin{array}{l}\text { HEATING } \\
\text { SAVINGS }\end{array}$ & $\begin{array}{l}\text { COOLING } \\
\text { SAVINGS }\end{array}$ & SÄVİNĞ & INSULT. & SAVINGS & SAVINGS \\
\hline & $\begin{array}{r}1346 \\
\$ \quad 140 \\
\$ \quad 746\end{array}$ & $\$$ & $\begin{array}{r}1346 \\
148 \\
\quad 746\end{array}$ & $\begin{array}{l}\$ 101 \\
\$ 268 \\
\$ 1\end{array}$ & $\begin{array}{r}1245 \\
-128 \\
\$ \quad 578\end{array}$ & $\begin{array}{l}1245 \\
1116 \\
1695\end{array}$ \\
\hline
\end{tabular}

\begin{tabular}{|c|c|c|c|c|c|c|c|c|c|}
\hline $\begin{array}{l}\text { ELECTRIC } \\
\text { ELECTRIC }\end{array}$ & $\begin{array}{l}\text { FURNACE } \\
\text { AIR COND. }\end{array}$ & $e^{\$}$ & $\$ 04$ & $\begin{array}{l}K W H \\
O / K W H\end{array}$ & $\vdots$ & $\begin{array}{l}\text { PWF } \\
\text { PWF }\end{array}$ & $\begin{array}{l}= \\
=\end{array}$ & $\begin{array}{l}18.215 \\
18.215\end{array}$ & \multirow[b]{2}{*}{$\begin{array}{c}\text { CUMULATIVE } \\
\text { NET } \\
\text { SAVINUS }\end{array}$} \\
\hline $\begin{array}{l}\text { INSULATION } \\
\text { SPECS }\end{array}$ & $\begin{array}{l}\text { IN } \\
\text { SAVATING }\end{array}$ & $\begin{array}{l}\text { ICREME } \\
\text { COOL } \\
\text { SAVI }\end{array}$ & $\begin{array}{l}\text { ENTAL } \\
\text { INGG } \\
\text { INGS }\end{array}$ & SAVINGS & & $\begin{array}{l}\text { NSUL. } \\
\text { COST }\end{array}$ & & SAVINGS & \\
\hline $\begin{array}{l}\text { B3 } \\
\text { D3 } \\
\text { F2 }\end{array}$ & $\begin{array}{r}2607 \\
272 \\
1446\end{array}$ & $\$$ & $\begin{array}{l}0 \\
0 \\
0\end{array}$ & $\begin{array}{r}2607 \\
\$ 272 \\
\$ 1446\end{array}$ & & $\begin{array}{l}101 \\
269 \\
168\end{array}$ & & $\begin{array}{l}2506 \\
1278\end{array}$ & $\begin{array}{l}2506 \\
2510 \\
3788\end{array}$ \\
\hline
\end{tabular}

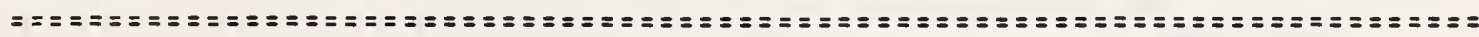
HEAT PUMP
ELECTRIC AIR COND. e \$ $\$ .040 / K W H$
PWF

INCREMENTAL

\begin{tabular}{|c|c|c|c|c|c|c|}
\hline $\begin{array}{l}\text { NSULATION } \\
\text { SPECS }\end{array}$ & $\begin{array}{l}\text { HEATINC } \\
\text { SAVINGS }\end{array}$ & $\begin{array}{l}\text { COOLING } \\
\text { SAVINGS }\end{array}$ & SAVINGS & INSUL. & SAVINGS & SAVITEG \\
\hline & $\begin{array}{r}1682 \\
175 \\
933\end{array}$ & $\begin{array}{ll}\$ & 0 \\
& 0\end{array}$ & $\begin{array}{r}1682 \\
\$ \quad 175 \\
\$ \quad 933\end{array}$ & $\begin{array}{l}101 \\
269 \\
2168\end{array}$ & $\begin{array}{r}1581 \\
-765 \\
\$\end{array}$ & $\begin{array}{r}1581 \\
\$ 1488 \\
2253\end{array}$ \\
\hline
\end{tabular}


HUD2

PHOENIX ARIZONA 200 SO FT HOUSE 90 DEGREE ROTATION (A CASE) COOLING LOADS BASED ON NO COOLING SAVINGS

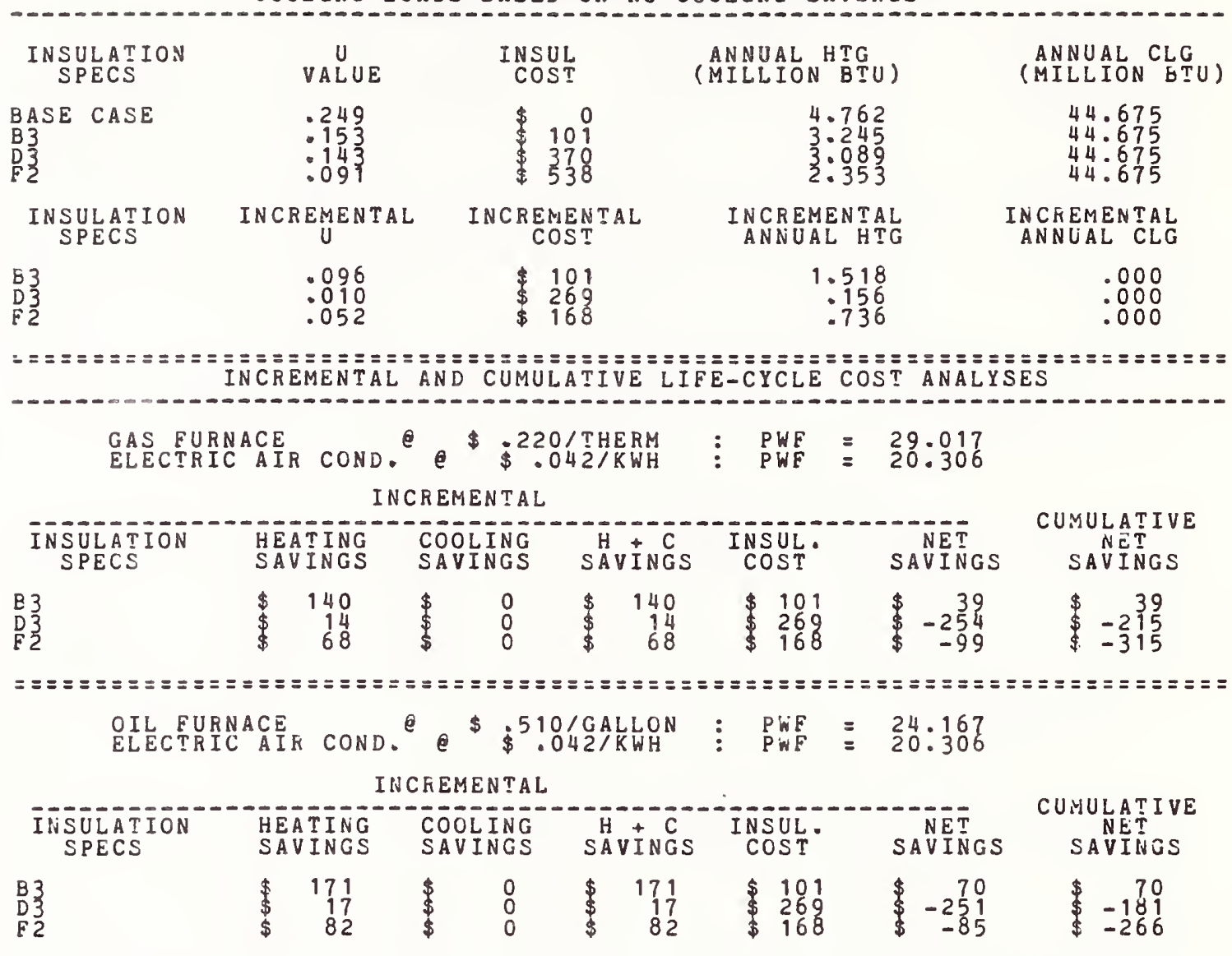

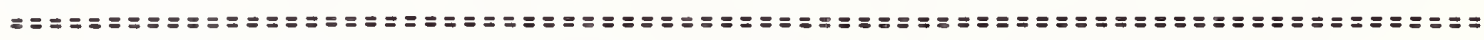
ELECTRIC FURNACE
ELECTRIC AIR COND. \$ $\$ .042 / K W H$
$\$ 042 / K W H$ INCREMENTAL

\begin{tabular}{|c|c|c|c|c|c|c|c|}
\hline $\begin{array}{l}\text { IN SULAT ION } \\
\text { SPECS }\end{array}$ & $\begin{array}{l}\text { HEATING } \\
\text { SAVINGS }\end{array}$ & $\begin{array}{l}\text { COOLING } \\
\text { SAVINGS }\end{array}$ & $\stackrel{H}{\text { SAVIINGS }}$ & $\begin{array}{l}\text { INSUL. } \\
\text { COST }\end{array}$ & SAVINGS & $\begin{array}{r}\text { CUMU } \\
\text { SA }\end{array}$ & $\begin{array}{l}\text { LAT IVE } \\
\text { NET } \\
\text { VINGS }\end{array}$ \\
\hline & $\begin{array}{l}379 \\
\$ \quad 18 \\
\$ \quad 183\end{array}$ & $\$$ & $\begin{array}{l}379 \\
38 \\
183\end{array}$ & $\begin{array}{l}\$ 101 \\
\$ 269 \\
\$ 168\end{array}$ & 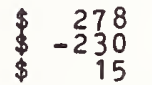 & $\$$ & $\begin{array}{r}278 \\
48 \\
64\end{array}$ \\
\hline
\end{tabular}

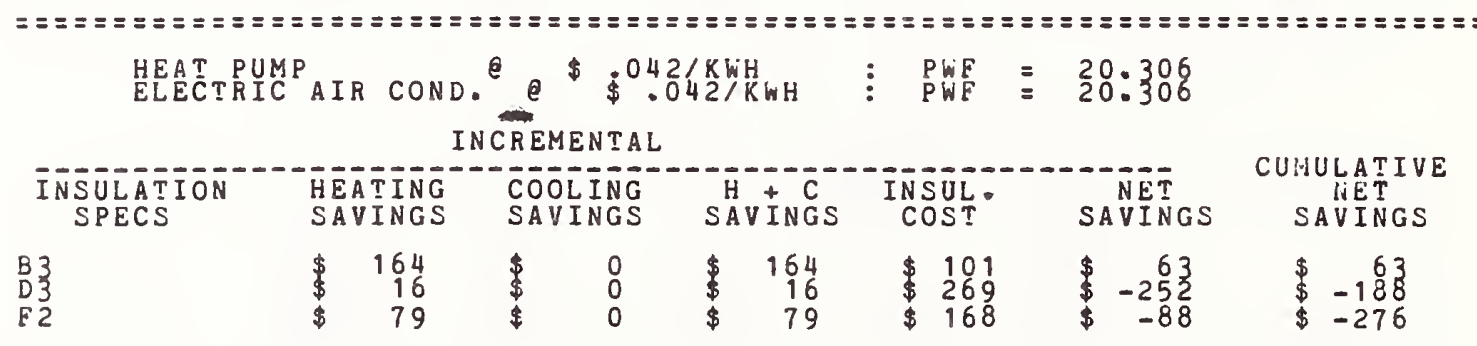


NBS-114A (REV. $9-78)$

\begin{tabular}{|c|c|c|c|}
\hline $\begin{array}{l}\text { U.S. DEPT. OF COMM. } \\
\text { BIBLIOGRAPHIC DATA } \\
\text { SHEET }\end{array}$ & $\begin{array}{l}\text { 1. PUBLICATION OR REPORT NO. } \\
\text { NBSIR } 79-1789\end{array}$ & $2,60 \mathrm{CH}$ Acosisien $\mathrm{No}$ & K\% \\
\hline \multirow{2}{*}{\multicolumn{3}{|c|}{$\begin{array}{l}\text { 4. TITLE AND SUBTITLE } \\
\text { Economic Analysis of Insulation in Selected Masonry } \\
\text { and Wood-Frame Walls }\end{array}$}} & $\begin{array}{l}\text { 5. Publication Date } \\
\text { September } 1979\end{array}$ \\
\hline & & & Proming Optization Code: \\
\hline \multicolumn{3}{|c|}{$\begin{array}{l}\text { 7. AUTHOR(S) } \\
\text { Stephen R. Petersen }\end{array}$} & 8. Performing Organ. Report No. \\
\hline \multirow{2}{*}{\multicolumn{3}{|c|}{$\begin{array}{l}\text { NATIONAL BUREAU OF STANDAF } \\
\text { DEPARTMENT OF COMMERCE } \\
\text { WASHINGTON, DC } 20234\end{array}$}} & 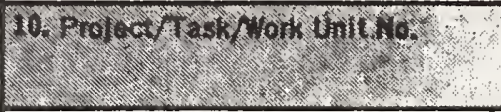 \\
\hline & & & 11. Contract/Grant No. \\
\hline \multirow{2}{*}{\multicolumn{3}{|c|}{$\begin{array}{l}\text { 12. SPONSORING ORGANIZATION NAME AND COMPLETE ADDRESS (Streot, City, Stato, ZIP) } \\
\text { U.S. Department of Housing and Urban Development } \\
4517 \text { th Street, SW } \\
\text { Washington, D.C. }\end{array}$}} & $\begin{array}{l}\text { 13. Type of Report \& Period Covered } \\
\text { FINAL }\end{array}$ \\
\hline & & & 26. Skonsoring A gefor Code \\
\hline
\end{tabular}

15. SUPPLEMENTARY NOTES

Document describes a computer program; SF-185, FIPS Software Summary, is attached.

16. ABSTRACT (A 200-word or less factual summary of most slgnificant in formation. If document includes a signlficant blbliography or literature survey, mention it here.)

This report provides a life-cycle cost-benefit analysis of several alternative methods for insulating $8^{\prime \prime}$ (200 mm) concrete masonry walls in new single-family residences. In addition, a cost-benefit analysis for insulation in wood-frame

ralls is provided, consistent with the assumptions used in the masonry wall analysis. A dynamic load simulation mode1, NBSLD, was used to calculate the heating and cooling requirements for a 1176 square foot $\left(110 \mathrm{~m}^{2}\right)$ house with different levels of thermal resistance for both wal1 types in eight geographic locations. These data are used to calculate the reduction in annual heating and cooling requirements due to several different types of insulation in the cores and furring spaces of the masonry wall and the cavities of the wood-frame wall.

Economic analysis is applied to determine estimates of life-cycle savings from insulation for different locations and furnace types in order to determine the most cost-effective insulation level. In general it is found that the maximum economically justifiable level of insulation in the masonry wall is considerably lower than for the wood-frame wall because of the significantly higher cost of insulating masonry walls.

17. KEY WORDS (six to twelve entries; alphabetical order; capitalize onty the first letter of the first key word unless a proper name; separated by semicolons) Building economics; energy conservation; insulation; 1ife-cycle cost analysis; masonry construction; space heating and cooling requirements; thermal mass.

\section{AVAILABILITY \\ [X] Unlimited}

For Official Distribution. Do Hot Release to NTIS

Order From Sup. of Doc., U.S. Government Printing Office, Wasinington, DC 20402, SD Stock No. SNO03-003-

[X] Order From National Technical Information Service (NTIS), Springfield, VA. 22161

\begin{tabular}{|l|c|}
\hline $\begin{array}{l}\text { 19. SECURITY CLASS } \\
\text { (THIS REPORT) } \\
\text { UNCLASSIFIED }\end{array}$ & $\begin{array}{c}\text { 21. NO. OF } \\
\text { PRINTED PAGES } \\
120\end{array}$ \\
$\begin{array}{l}\text { 20. SECURITY CLASS } \\
\text { (THIS PAGE) } \\
\text { UNCLASSIFIED }\end{array}$ & \begin{tabular}{c} 
22. Price \\
\hline
\end{tabular} \\
\hline
\end{tabular}




เ 